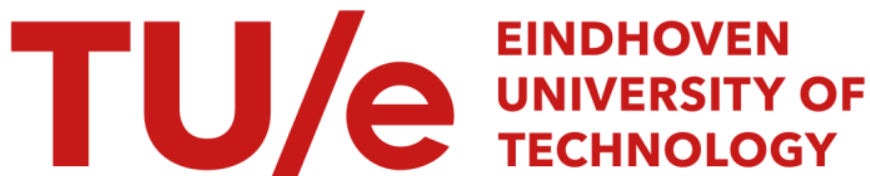

\section{Modelling strategic behaviour in anticipation of congestion}

\section{Citation for published version (APA):}

Han, Q. (2006). Modelling strategic behaviour in anticipation of congestion. [Phd Thesis 1 (Research TU/e / Graduation TU/e), Built Environment]. Technische Universiteit Eindhoven. https://doi.org/10.6100/IR607727

DOI:

10.6100/IR607727

Document status and date:

Published: 01/01/2006

\section{Document Version:}

Publisher's PDF, also known as Version of Record (includes final page, issue and volume numbers)

\section{Please check the document version of this publication:}

- A submitted manuscript is the version of the article upon submission and before peer-review. There can be important differences between the submitted version and the official published version of record. People interested in the research are advised to contact the author for the final version of the publication, or visit the $\mathrm{DOI}$ to the publisher's website.

- The final author version and the galley proof are versions of the publication after peer review.

- The final published version features the final layout of the paper including the volume, issue and page numbers.

Link to publication

\section{General rights}

Copyright and moral rights for the publications made accessible in the public portal are retained by the authors and/or other copyright owners and it is a condition of accessing publications that users recognise and abide by the legal requirements associated with these rights.

- Users may download and print one copy of any publication from the public portal for the purpose of private study or research.

- You may not further distribute the material or use it for any profit-making activity or commercial gain

- You may freely distribute the URL identifying the publication in the public portal.

If the publication is distributed under the terms of Article 25fa of the Dutch Copyright Act, indicated by the "Taverne" license above, please follow below link for the End User Agreement:

www.tue.nl/taverne

Take down policy

If you believe that this document breaches copyright please contact us at:

openaccess@tue.nl

providing details and we will investigate your claim. 


\section{Modelling Strategic Behaviour in Anticipation of Congestion}

\section{PROEFSCHRIFT}

ter verkrijging van de graad van doctor aan de

Technische Universiteit Eindhoven, op gezag van de Rector Magnificus, prof.dr.ir. C.J. van Duijn, voor een commissie aangewezen door het College voor

Promoties in het openbaar te verdedigen op woensdag 22 maart 2006 om 16.00 uur

door

Qi Han

geboren te Nanjing, China 
Dit proefschrift is goedgekeurd door de promotoren:

prof.dr. H.J.P. Timmermans

en

prof.dr. W.F. van Raaij

Copromotor:

prof.dr.ir. B.G.C. Dellaert

Copyright () 2006 Q. Han

Technische Universiteit Eindhoven,

Faculteit Bouwkunde, capaciteitsgroep Stedebouw

Cover design: Tekenstudio, Faculteit Bouwkunde

Printed by the Technology University of Eindhoven Press Facilities 


\section{Preface}

In this thesis, a approach is developed to explore and describe strategic choice behaviour in anticipation of congestion, based on interaction and interdependencies between individuals and between individuals and service providers. It formulates the process of how people would arrive at choices in a congested environment and how information or guidance would become effective towards serving a certain goal. My research interest in this area emerged during my master study. When I first saw the research proposal of this Ph.D. project a few years ago, I knew that I found something that would suit me.

The research project was sponsored by SOBU: a collaboration between Tilburg University and Eindhoven University of Technology. The original proposal was developed by Harry Timmermans and Benedict Dellaert when he still was in Tilburg University. Soon after the proposal was funded, he became a professor at Maastricht University and Fred van Raaij joined the team. However, Benedict stayed heavily involved in the project, and hence I was lucky to have such an active scholarly environment. The available travel grant provided me also great opportunities to attend international conferences and meet other researchers. Besides, many people have supported me in conducting the research reported in this thesis. I wish to acknowledge all the help that they given. There are some that I would like to mention specifically for their contributions. 
Professor Harry Timmermans, my first supervisor, has supported me in many ways. With his special style of open guidance he knows how to encourage people to work independently, to develop their own expertise and to pursue their research goals. This and his strategic insights in the world of science have helped me in not only developing the line of my research interest, but also offering me a wonderful opportunity to learn how to conduct research and publish in international journals. Besides, his kind and careful comments on the draft version of my thesis were very important.

Many thanks also go to Professor Fred van Raaij, my second supervisor. He succeeded Professor Benedict Dellaert and continued bridging the cooperation between the two universities on this project. He introduced me the educational credit system in Tilburg University that I could use to get sufficient respondents for the data collection. From preparing the recruit announcement, arranging the laboratory, to credit registration afterwards, he has given me invaluable support in two experimental data collections in my research project. Besides, his insightful reviews gave valuable contributions to my work.

I also owe much to Professor Benedict Dellaert, my co-promotor. He was my second supervisor for the first half year before he took the job position at Maastricht University. His contribution to my research however never stopped. His ability to find creative solutions for complicated problems and his efforts to discuss my work at the most detailed level are much appreciated. His suggestions and helpful comments on my work have improved the quality of my research significantly.

During the years, my colleagues in the urban planning group supported me in many different ways. I learned many things from working with them and I feel honoured simply to be surrounded by them. Although it is difficult to put in words, I want to take this opportunity to express my appreciation to all of them, in particular to Aloys Borgers, Theo Arentze, Peter van der Waerden and Astrid Kemperman for helping me out in explaining models and software, Leo van Veghel for providing me access to valuable references, and both Mandy van de Sande - van Kasteren and Anja Janssen for their excellent secretarial assistance and care. Also I enjoyed a lot the discussions with former group members Manon van Middelkoop, Chang-Hyeon Joh, Maarten Ponje and current Ph.D. candidates. Among them, a special word of thanks goes to Dick Saarloos, who shared the office with me for the last few years, for the interesting talks on research and cultural difference, and for his help in times of need.

Finally, I would like to thank my husband, Hai. His support has been a constant encouragement. Last but not least, I also like to thank my parents, who have always been there for me with their unconditional care.

Qi Han

Eindhoven, December 2005 


\section{Contents}

Preface iii

List of figures $\quad$ ix

List of tables $\quad x$

1 Introduction 1

2 Modelling spatial choice behaviour in urban planning 5

2.1 Introduction 5

2.2 Individual choice models 6

2.2.1 Spatial interaction modelling 6

2.2.2 Discrete choice modelling 7

2.2.3 Activity-based modelling 9

2.2.4 Evaluation 9

2.3 Choice behaviour in response to information 10

$\begin{array}{ll}\text { 2.3.1 Information use and provision } & 10\end{array}$

$\begin{array}{ll}2.3 .2 \text { Evaluation } & 11\end{array}$

2.4 Choice behaviour in response to other individuals' behaviour 12

2.4.1 Interdependence within multi-person households 12 (intra household)

2.4.2 Interdependence within the social-spatial network 12 (inter household)

2.4.3 Game theory and interactive decision making 14

2.4.4 Game theory and discrete choice models 15

$\begin{array}{lll}2.4 .5 & \text { Evaluation } & 15\end{array}$

$\begin{array}{lll}2.5 & \text { Conclusion and discussion } & 16\end{array}$

3 Theory 17

$\begin{array}{lll}3.1 & \text { Introduction } & 17\end{array}$

3.2 Models of strategic choice behaviour based on interaction and 18 interdependencies between individuals 
3.2.1 N-player $J$-option game formulation $\quad 18$

3.2.2 Strategic discrete choice model 22

3.2.2.1 Specification of the exogenous component 22

3.2.2.2 Specification of the endogenous component 23

3.2.2.3 Specification of the disturbance 24

3.2.3 Equilibrium conception: consistence condition 24

3.2.3.1 Bayesian Nash equilibrium 25

3.2.3.2 Quantal response equilibrium 27

3.2.3.3 Correspondence between QRE and BNE 28

3.2.4 The logit approach $\quad 30$

3.2.4.1 The error parameter 31

3.2.4.2 Estimation issues 33

3.2.5 Inconsistence: imperfect expectation in one-shot situation 35

3.2.6 Interpretation, application 37

3.2.6.1 Understanding mixed-motives 37

3.2.6.2 Application issues: user, domain and data 37

3.3 Models of strategic choice behaviour based on interaction and $\quad 38$ interdependencies between individuals and information providers

3.3.1 Two-player two-stage game formulation 39

3.3.2 Interactive decisions between individuals and information 41 providers

3.3.3 Update of individual's expectation given information providers' 42 recommendation

3.3.4 Information provider's objective 44

3.4 Conclusion and discussion $\quad 46$

4 Numerical simulation $\quad 49$

4.1 Introduction 49

4.2 Interactive decisions between individuals $\quad 50$

4.2.1 Algorithm $\quad 51$

4.2.2 Simulation results $\quad 51$

4.2.2.1 Sensitivity to choice alternative facets $\quad 52$

4.2.2.2 Sensitivity to interaction 53

4.2.2.3 Sensitivity to uncertainty of conjectures 54

4.3 Interactive decisions between individuals and an information 55 provider 
4.3.1 Algorithm 56

4.3.2 Simulation results $\quad 57$

4.3.2.1 Effects of different objectives of the information 58 provider

4.3.2.2 Effects of different levels of individuals' conjecture $\quad 60$ ability

4.4 Conclusion and discussion

5 Timing choice $\quad 65$

$\begin{array}{llr}5.1 & \text { Introduction } & 65\end{array}$

5.2 Hypothesis 66

$\begin{array}{lll}5.3 & \text { Timing choice data } & 67\end{array}$

$\begin{array}{lll}\text { 5.3.1 Data collection } & 67\end{array}$

5.3.2 Dinner timing choice 68

5.3.3 Participation timing choice $\quad 69$

5.3.4 Structure of the experimental design 70

5.4 Analysis of timing choice data $\quad 71$

5.4.1 Dinner timing choice model 71

5.4.2 Participation timing choice model 77

5.4.3 Non-strategic and strategic behaviour 79

5.4.4 Test of hypothesis $\quad 84$

$\begin{array}{lll}5.5 & \text { Conclusion and discussion } & 88\end{array}$

$6 \quad$ Repeated strategic choice $\quad 89$

$\begin{array}{llr}6.1 & \text { Introduction } & 89\end{array}$

$\begin{array}{lll}6.2 & \text { Research question } & 90\end{array}$

$\begin{array}{ll}\text { 6.3 Repeated strategic choice data } & 92\end{array}$

6.3.1 Data collection 92

6.3.2 Structure of the experimental design 93

6.3.3 Implementation 94

6.3.4 Process 95

6.4 Analysis of repeated strategic choice data 98

6.4.1 General results: performance 98

6.4.2 General results: choice frequencies 101

$\begin{array}{ll}\text { 6.4.3 Regression model estimation } & 103\end{array}$ 
6.4.4 Choice model estimation 106

6.4.4.1 Strategic choice model 106

6.4.4.2 Simulation for projecting the anticipated congestion 108

$\begin{array}{ll}\text { 6.4.4.3 Results for one-shot situations } & 108\end{array}$

6.4.4.4 Results for repeated situations 111

6.4.4.5 Results about learning in situations without 113

6.4.4.6 Results about learning in situations with 115 recommendation

$\begin{array}{lll}\text { 6.5 Conclusion and discussion } & 118\end{array}$

7 Conclusion and discussion $\quad 121$

$\begin{array}{lll}7.1 & \text { Introduction } & 121\end{array}$

$\begin{array}{lll}7.2 & \text { Short summary of the study } & 122\end{array}$

$\begin{array}{lll}7.3 & \text { Discussion of the proposed modelling approach } & 124\end{array}$

$\begin{array}{ll}7.4 & \text { Avenues for future research } \\ \end{array}$

$\begin{array}{ll}\text { Bibliography } & 129\end{array}$

$\begin{array}{lll}\text { Appendix A-1 } & \text { Announcement of timing choice experiment }\end{array}$

$\begin{array}{lll}\text { Appendix A-2 } & \text { Questionnaire of timing choice experiment }\end{array}$

$\begin{array}{lll}\text { Appendix B-1 Announcement of repeated choice experiment } & 147\end{array}$

$\begin{array}{lll}\text { Appendix B-2 Overview of the tasks } & 148\end{array}$

$\begin{array}{lll}\text { Appendix B-3 Screen shot of the instruction } & 149\end{array}$

$\begin{array}{lll}\text { Appendix B-4 Screen shot of the tips } & 150\end{array}$

$\begin{array}{lll}\text { Appendix B-5 } & \text { A screen shot after subjects made a choice } & 151\end{array}$

$\begin{array}{ll}\text { Author index } & 153\end{array}$

$\begin{array}{ll}\text { Subject index } & 157\end{array}$

$\begin{array}{ll}\text { Samenvatting (Dutch summary) } & 161\end{array}$

$\begin{array}{ll}\text { Curriculum vitae } & 163\end{array}$ 


\section{List of figures}

Figure 3-1 Game 1 formulation 20

Figure 3-2 Game 1 extensive form 21

Figure 3-3 Relation between type and strategy 29

Figure 3-4 Error parameter effect 32

Figure 3-5 Game 2 formulation 40

Figure 3-6 Game 2 extensive form 40

Figure 3-7 Effect of self-expectation on compliance rate 44

Figure 4-1 Choice alternative impact 53

Figure 4-2 Interaction impact $(\theta) \quad 54$

Figure 4-3 Uncertainty impact 55

Figure 4-4 Results for the Nash equilibrium 58

Figure 4-5 Results for the quantal response equilibrium 59

Figure 4-6 Results for the noisy introspection model 60

$\begin{array}{lll}\text { Figure 4-7 Results for the equal usage objective } & 61\end{array}$

Figure 4-8 Results for the welfare maximum objective 61

Figure 4-9 Results for the user equity objective 62

Figure 5-1 Structure of timing choice experimental design 71

Figure 5-2 Simulations of participation timing choice 86

Figure 6-1 Structure of two dimensional effects 91

Figure 6-2 A screen shot of choice task 97

Figure 6-3 Average performance with and without recommendation 98

Figure 6-4 Average performance with recommendation for different 99

Figure 6-5 Average performances for the certainty of others' preference 100

Figure 6-6 Recommendation effect in choice frequencies 101

Figure 6-7 Preference certainty effect in choice frequencies 102

Figure 6-8 Learning effect in the no recommendation situation 114

Figure 6-9 Learning effect in the with recommendation situation 115

Figure 6-10 Learning effect for the marginal disutility of congestion 117

Figure 6-11 Learning effect for the certainty of others' preference 118 


\section{List of tables}

Table 5-1 Overview of registration for timing choice experiment 67

Table 5-2 Non-strategic dinner timing choice estimates (1-A) 72

Table 5-3 Strategic dinner timing choice estimates (1-B) 74

Table 5-4 Dinner timing choice model estimates (1-A \& 1-B) 76

Table 5-5 Participation timing choice estimates (2-B \& 2-C) 78

Table 5-6 Observed participation timing choice frequencies 78

Table 5-7 Non-strategic behaviour estimates (1-A \& 2-B) 79

Table 5-8 Non-strategic behaviour contrast parameter estimates (1-A \& 2-B) 80

Table 5-9 Strategy behaviour estimates (1-B \& 2-C) 81

Table 5-10 Strategy behaviour contrast parameter estimates (1-B \& 2-C) 82

Table 5-11 Information impacts (2-C) 83

Table 5-12 Simulations of participation timing choice 86

Table 6-1 Overview of registration for repeated strategic choice 92

Table 6-2a Regression model estimates $\left(1^{\text {st }}\right.$ round vs. $13^{\text {th }}$ round) 104

Table 6-2b Test statistics for the classical regression model 104

Table 6-3 Contrast parameter estimates $\left(1^{\text {st }}\right.$ round vs. $13^{\text {th }}$ round $) \quad 105$

Table 6-4 One-shot situation strategic choice model estimates ( $1^{\text {st }}$ round) 109

Table 6-5 One-shot situation MNL model log likelihoods ( $1^{\text {st }}$ round data) $\quad 110$

Table 6-6 Repeated situation strategic choice model estimates ( $13^{\text {th }}$ round) 112

Table 6-7 Repeated situation MNL model log likelihoods (13 ${ }^{\text {th }}$ round data) 113 


\section{Chapter 1}

\section{Introduction}

Recent decades have witnessed a tendency towards individualization in society at large. Business strategies and marketing efforts have shown a shift from mass production to flexible individual service provision, although it may be argued that there are limits to such individualization (Dellaert and Stremersch, 2003). In most cases, many industries have shown a greater sensitivity to individual preferences and individual demand, which brings diversification in goods and services and implies a partner relationship between producer and consumer.

Although relatively late, local government agencies, including urban planning authorities, have followed this trend and have become more conscious about consumer preferences in delivering their services and even have initiated new activities in an attempt to bring new visitors and residents to their city. A good example is the introduction of the concept of city marketing (Paddison, 1993) and destination marketing (Kotler, 2000). These concepts encompass different ideas, but relate specifically to the attempt that cities are trying to improve the consumer's image of their city and to lead them to visit more facilities in their city. In that sense, the concept is often linked to urban tourism and leisure. This shift in focus, or perhaps better, this additional concern of urban planning agencies, has implied a widening of the scope of their activities.

Traditionally, one of the key tasks of urban planning and marketing research in this context has been to predict consumer response to new service provision. Various types of models have been developed over time to predict the percentage of some target 
population that will use the new or revised facility. These predictions are then used to evaluate alternative policies, assess the feasibility of new development or predict the impact of such policies on existing facilities. Such models have been applied with varying degrees of success. Most of these models share the property that they have been developed to predict the long-term impact of new facilities on consumer demand, primarily as a function of the locational and non-locational characteristics of the facilities and socio-demographics, assuming explicitly or implicitly (quasi-)equilibrium and unconstrained capacity.

As consumer life-styles are becoming increasingly demanding, however, the underlying assumption of unconstrained capacity of facilities seems inappropriate especially when viewed in light of urban sustainable development. Many service providers (both public and private) are facing limitations in their ability to serve all their customers at their most preferred time. For example, museums, theatres, tourist attractions but also parking lots and roads typically are faced with constraints in terms of how many consumers can be accommodated during the most popular time slots. Recreational facilities such as sports facilities and urban parks, and professional services such as dentists may also experience periods in which demand exceeds supply (e.g., before and after work or school hours). The same can be observed for information technology based services such as call centres and customer service desks, and even Internet-based services have been known to break down if demand exceeds supply when certain websites become extremely popular, literally overnight.

Whereas urban planning authorities were traditionally concerned with the planning and design of new land uses for expanding physical capacity, increasingly effort has been put on optimal control and use of existing facilities, often involving collaboration between the public and private sector. Such optimal control attempts to influence individual's behaviour such as to optimize the use of the available facilities and optimize the value of consumers' experience. Information provision channels such as websites, location-based services and guidance systems are all examples of this trend and it is to be expected that their application and importance will increase with respect to many issues related to urban planning and city marketing. Moreover, the shift in attention to demand management has led to a growing interest of researchers in developing new models for predicting consumption distributions across time and space that can support the evaluation of information provision in terms of its expected effect on individual's behaviour and its consequence for the urban economy.

Consumers share similar timing preferences for many services, due to common time constraints such as working hours, public holidays, etc. Thus, strong variations in demand over time for capacity-constrained services typically have unpleasant consequences, such as increased waiting times or less comfortable and enjoyable service experiences for those consumers who wish to use the service at the most popular times. We refer to this phenomenon as congestion.

Under these circumstances, consumers may exhibit strategic choice behaviour. If certain time slots are more popular than others, consumers make a strategic trade-off: should they choose their preferred slot (and most likely that of others), or should they avoid congestion and choose an alternative slot? This trade-off requires anticipating other consumers' choices. That is, they will choose particular choice options taking into account their expectations of the behaviour of others such as to maximize their own utility. For example, the utility of visiting leisure facilities (restaurants, urban parks, movie theatres) may depend on the number of other visitors, depending on one's 
preferences about crowding. Similarly, the utility that is derived from conducting a shopping activity at a particular location may depend on the queues that one experiences. While this type of consumer behaviour might be less relevant in terms of the long-term impact of new facilities, it is of critical importance in better understanding short-term responses and improving control policies.

As indicated, the issue how consumers respond strategically to congestion has not received a lot of attention in the literature on consumer choice models. Congestion in capacity-constrained services is essentially the negative outcome at an aggregate level of choices that individuals make in self-interest while assuming that others do the same. These choices made by individuals lead to aggregate, macro-level service consumption patterns, which in turn influence individual, micro-level, decisions. The equilibrium consumption-timing pattern is then determined by the trade-off between timing preference and anticipation of congestion.

Control strategies and technologies such as web services, location-based services and personal travel assistants make it possible and easier for individuals to inform themselves about the state of the system, reducing uncertainty, while they are trying to anticipate likely choices of others. Likewise, using such technologies, service providers (urban authorities, business consortia, etc.) can reach their (potential) customers through information provision. One of the demand management methods thus is to provide information to induce individuals to behave in a certain way such that congestion can be better controlled and some system-level objective is better satisfied. When such information is provided, consumers need to make assumptions whether and how other individuals will adjust their current behaviour, while information providers need to anticipate the consumers' reactions to the information they offer. That is, both service providers and consumers may act strategically since they need to anticipate others' decisions, while making their own decision.

The aim of this thesis is to develop a model of such strategic behaviour based on the interaction and interdependencies both between individuals and between individuals and information providers. This model will have the potential to assist planning authorities and service providers in general to better control the use of existing facilities. The aim, however, is not to develop a fully operational, specific model, for a specific type of service, but rather to explore and investigate particular classes of strategic choice models. In that sense, any application will require additional operational decisions, additional data collection and model estimation.

In order to develop these models, the following research questions, which serve as building blocks in the development of the choice models, were formulated to guide the project:

- How do individuals incorporate strategic considerations regarding possible service congestion in their service consumption timing choice?

- How do individuals incorporate strategic considerations under guidance information regarding possible service congestion in their service consumption timing choice?

- How do information providers incorporate strategic considerations regarding the reaction of individuals to achieve certain objectives in their information provision? 
This thesis reports the development of models of strategic choice behaviour and provides an answer to the formulated research questions. The thesis is organized into seven chapters. Following this introductory chapter, Chapter 2 provides a brief review of the relevant literature on models of consumer choice behaviour. Rather than giving a comprehensive in-depth review of individual choice models and game theory, this chapter focuses on previous research in the individual choice and game theory literature in an attempt to discuss some key concepts and to put this study into the wider literature such that readers can appreciate the specific contribution of this study to the current state of the art.

Based on this brief literature review, Chapter 3 then discusses the proposed models of strategic choice behaviour. It starts with the fundamentals of game theory. On the basis of these fundamentals, a framework for modelling strategic choice behaviour is developed. First, models of individuals' conjectures about the strategic behaviour of other individuals are discussed. Three levels of anticipation are explored to reflect three typical situations: (1) expectation with complete information in ideal situations - Nash equilibrium, (2) expectation with incomplete information in repeated situations - quantal response equilibrium, and (3) expectation with incomplete information in one-shot situations - noisy introspection. Next, this is extended to the problem of interactive decisions between individuals and information providers, assuming different objectives that different kinds of information providers may have. This is followed in Chapter 4 by a series of numerical computer simulations that were conducted to examine the properties of the proposed models. In these studies, alternative parameter settings were used to investigate the face validity of the models of strategic choice behaviour.

Having provided evidence of face validity, the next step was to further test the models using empirical data. Because aspects of strategic behaviour are very difficult to disentangle using data of revealed choices in real world settings, two controlled experiments, involving several sub-experiments, were conducted. Chapter 5 reports the design, data collection and analysis results of the first experiment. This experimental study was designed to examine how individuals incorporate strategic considerations with respect to congestion in their timing decisions in one-shot situations, using different application contexts. Chapter 6 describes the second study that involved a controlled computer experiment to examine whether learning takes place in strategic contexts and how consumers react to different information provision with different underlying objectives. This second study provides answers to the research questions about the interaction between individuals and information providers from one-shot to repeated situations.

Chapter 7 then concludes the thesis. The chapter summarizes the modelling efforts and the results of the analyses. This is followed by a discussion of the interpretation and implication of the results. The chapter ends with a discussion of the limitations and possible extensions of the models. 


\section{Chapter 2}

\section{Modelling Spatial Choice Behaviour in Urban Planning}

\subsection{Introduction}

Models of spatial choice behaviour have been around in urban planning for decades to assess the feasibility of planning actions or to predict external (competition) effects on existing destinations. Over the years, many different concepts and approaches have been developed and applied. The majority of the existing modelling techniques can be classified into two groups. The first group consists of the quantitative modelling approaches, which assume that individuals always select the alternative that has the maximum utility (Ben-Akiva and Lerman, 1985). This group ranges from the revealed choice and the stated choice models based on the multinomial logit (MNL) model to the more advanced nested-logit and mixed logit models. The second group consists of qualitative modelling approaches, using if-then Boolean logic and choice heuristics. This group ranges from production systems to decision tables and decision trees. ${ }^{1}$

\footnotetext{
${ }^{1}$ In addition to these major streams of choice models in urban planning, a multitude of specific models has been applied and suggested. These include, for example, search models and attitudinal models. Because there is no evidence of a consistent accumulation of interest in such models in planning applications, we do not discuss these here in any detail. Spatial interaction models continue to be popular, but in many applications have the same properties of the utility-maximizing discrete choice models, and hence we taken them as a starting point of our discussion in the next section.
} 
The evolution of these models witnesses a trend of increasing the complexity of these models by examining various types of interdependencies. Two forms of interdependence in spatial choice behaviour are of particular interest. First, interdependence exists among sequences of short-term activity-travel choices, and among events separated in space and time in the longer term. Acknowledging this, discrete choice models have evolved from modelling single facets to multiple facets of activitytravel patterns, and from modelling static (cross-sectional) situations to dynamic, uncertain real life situations. Second, interdependence exists between individuals within households or within social-spatial network. Some researchers have recently started to address this issue.

In this chapter, we will briefly discuss the most relevant models and argue that interdependence and interaction between individuals has not received sufficient attention in these models. An examination of the relevant literature indicates that interactive decision making - game theory - is the most promising approach to integrate into individual choice models to handle interactions that involve strategic considerations. This discussion should serve as a background for positioning the models that will be developed in this thesis.

\subsection{Individual choice models}

Modelling spatial choice behaviour has received much attention in the areas of geography, urban planning, transportation and marketing, where individual choice probabilities have been aggregated for policy forecasting. The goal of this section is not to give an extensive review of the field. Interested readers are referred to for example Timmermans (1993) and Oppewal (1995). Rather, it will discuss in some detail previous research that is relevant to the problems that are addressed in this thesis.

\subsubsection{Spatial interaction modelling}

In the 1960 and 1970s, gravity-based spatial interaction and entropy-maximising models dominated the field (see, e.g., Wilson 1988 for an overview). These models were based on concepts of social physics and predicted aggregate, inter-zonal flows as a function of the attractiveness of the destination zone, a distance decay function and, depending on the specific model, balancing factors. Spatial interaction models have been widely applied to predict shopping behaviour, commuting behaviour, recreational behaviour, etc., and variations continued to appear in the literature (e.g., Baxter and Ewing 1981; Fortheringham, 1983; Guy, 1987).

In the 1970s, spatial interaction models were criticised for their lack of behavioural theory. Some authors tried to show that the mathematical specification of the model was consistent with particular theories of choice behaviour. However, the models have several fundamental drawbacks. The failure to disentangle consumer preference from spatial structure is the most critical one. In addition, the limited transferability of the estimated parameters to areas with a different geometry, and the inevitable influence of the zoning system reduces the validity of the models (Timmermans, 1993; Fotheringham and Wong, 1991). 


\subsubsection{Discrete choice modelling}

An alternative foundation for spatial interaction models emerged in the 1970s. Under certain conditions, the model is equivalent to so-called discrete choice models, which can be derived from different theories of individual choice behaviour that originated from econometrics and psychology (an early example is Richards and Ben-Akiva, 1974). In the 1980s and 1990s, discrete choice models (Ben-Akiva and Lerman, 1985) gradually replaced spatial interaction models (Timmermans, 1993, Crouch and Louviere, 2000). In some cases, this was not much more than a label in the sense that the model was still derived from aggregate data. However, other studies were truly based on individual-level data. These models gained much in popularity in different fields of application, recently leading to the Noble Prize awarded to Daniel McFadden in 2000.

Discrete choice theory or more specifically underlying random utility theory assumes that individuals compare a limited set of discrete choice alternatives and choose the alternative that maximises their utility. The set of choice alternatives called choice-set is determined by the individual's knowledge of his environment, and therefore, is individual-specific. In most derivations ${ }^{2}$ of discrete choice models, the utility value is decomposed into a structural and a stochastic component, as follows:

$$
U_{j}=V_{j}+\varepsilon_{j}
$$

The structural component $V_{j}$ is usually defined as an additive function of the attributes on which choice alternatives are compared (i.e., $V_{j}=\boldsymbol{\beta} \boldsymbol{x}_{j}$, where $\boldsymbol{\beta}$ is an unknown vector of attribute weights and $\boldsymbol{x}_{j}$ is a vector of attribute values). For example, when choosing a restaurant to visit, the quality of the food, the ambience of the restaurant, the travel time and the available parking facilities appear in most models as influential attributes. The $\varepsilon_{j}$ component is commonly referred to as the error or disturbance term and represents variation in perceived utility due to taste variation, stochastic preferences of individuals and measurement errors. ${ }^{3}$

Different types of models have been formulated dependent on the specific assumptions regarding the distribution of the error terms, as reviewed in for example Crouch and Louviere (2000). The most widely used model type is the multinomial logit (MNL) model, which is derived from the assumption that the error terms are independently and identically distributed and follow a Gumbel (or double exponential, or type I extreme value) distribution. According to this model, the probability that choice alternative $j$ is chosen from a given choice set $\boldsymbol{J}$ equals:

\footnotetext{
${ }^{2}$ The multinomial logit model can be derived in different ways and is consistent with different theories and interpretations of the parameters. Because such fine-tuning is not central to our discussion, we will discuss loosely the model and underlying theory as in most publications.

${ }^{3}$ The specific interpretation depends on the theory that is used, the level of aggregation of the data and estimation issues. Rarely, these topics are separated in the literature as they should, but rather it is typically implicitly or explicitly assumed that the error terms capture all these factors.
} 


$$
\operatorname{Pr}(j \mid j \in J)=P\left(U_{j} \geq U_{j^{\prime}} ; \forall j^{\prime} \in J ; j^{\prime} \neq j\right)=\frac{\exp \left(\mu V_{j}\right)}{\sum_{j^{\prime} \in J} \exp \left(\mu V_{j^{\prime}}\right)}
$$

where $\mu$ is a scaling factor which is typically but arbitrarily set to unity in single choice situations. The major advantage of the MNL model over the alternative probit model (based on a normal distribution of the error terms) is that it has a closed form, which can be solved analytically.

Based on the kind of data used to estimate the model, a distinction can be made between revealed and stated preference/choice models. Revealed preference/choice models are estimated from observed choices in real-world settings. Revealed data have several limitations. First, choice observations cannot be made if particular choice alternatives do not yet exist. Secondly, observed choice does not necessarily reflect maximized utilities or preferences. This is especially true in constrained choices situations. Finally, when collecting revealed choice data, researchers have no or limited a priori control over the covariance structure of the data. Under some extreme conditions, this means that explanatory variables (the attributes of $\boldsymbol{x}$ ) are strongly correlated so that their separate effects are hard to disentangle. Consequently, attribute elasticities may be totally wrong and predictions of the impact of new choice alternatives may be misleading. Stated preference/choice models represent an attempt of overcoming these problems. Different type of approaches can be identified, but the term stated preference/choice models (often called conjoint models in marketing) typically refer to the use of experimental design principles to create choice experiments for collecting data such that the properties of the data meet the necessary and sufficient conditions to estimate the model of interest. This is not to say that stated preference/choice models are flawless. They have their own specific problems, and especially the question whether choices expressed in experiments are consistent with every day decision-making has been the focus of continuing discussion of external validity, in which different scholars take fundamentally different positions and in which empirical evidence is not conclusive.

In a stated choice experiment, individuals are asked to make a choice, responding to hypothetical choice situations. Choice alternatives are often constructed as attribute profiles based on a balanced experimental design, such that the effects of attributes can be measured independently. The MNL model is widely used as a tool to estimate these attribute effects, which can be interpreted in terms of preference and used to predict the impact of new policies.

The MNL model assumes the error terms to be identically and independently distributed (IID property), which results in the so-called independence from irrelevant alternatives (IIA property). This property states that the odd of choosing a particular alternative from any pair of choice alternatives is independent of the existence and attributes of any other alternative in the choice set. This means that the degree of similarity between choice alternatives does not have any impact on choice probabilities and market shares, which is theoretically intuitive. The MNL model does not account for any systematic correlation between the error terms, which is one way of representing similarity between choice alternatives. Thus, the MNL model has been extended to derive more complex discrete choice models, including the nested logit model, the random parameter logit model, etc. These models relax the strict assumption underlying the MNL 
model by allowing for variances and covariances (e.g., Kamakura and Srivastava, 1984; Dellaert, et al., 1997a, 1998), and heterogeneity (e.g., Arentze, et al., 2005). Moreover, the basic model has been extended to incorporate cognitive and attitudinal processes, and different types of data (Ben-Akiva, et al., 1999; McFadden, 2001).

\subsubsection{Activity-based modelling}

In the mid 1990s, even more complex activity-based models started to appear in the literature. These models are based on the premise that spatial choice behaviour can be better understood in terms of the way in which individuals and households organise their activities in time and space (see Timmermans, et al., 2002 for an overview). This broader conceptualisation allows the simulation not only of direct but also of various kinds of indirect effects.

Recently, the activity-based approach has led to an overwhelming number of studies in transportation research and urban planning. In addition to studies on activity participation (e.g., Lu and Pas, 1997, 1999; Ma and Goulias, 1997), duration (e.g., Kitamura, 1984), task allocation (e.g., Golob and McNally, 1997), and timing (Mannering, et al., 1994; Polak, 1987), several comprehensive models of activity travel demand have been suggested. Utility maximizing nested logit models have been advanced from trip and tour based to activity-based models (e.g., Bowman and Ben-Akiva, 1999). Whereas their model is based on revealed preference data, the Cobra model (Wang and Timmermans, 2000) used experimental design data from conjoint experiments. Because the assumptions underlying the nested logit model were not always satisfied by the empirical data, Bhat and Koppelman (1993) and Borgers et al., (2002) developed suites of models, which predicted, in combination, multiple facets of activity-travel behaviour.

A second line of research attempted to avoid the rather rigorous assumptions underlying utility-maximizing models by exploring the feasibility of computational process models. Examples are Scheduler (Gärling, et al., 1989), AMOS (Pendyala, et al., 1998), Albatross (Arentze and Timmermans, 2000), and derived methodological work (e.g., Moons, et al., 2002). In addition, hybrid approaches have been suggested. An early example of a hybrid approach is Smash (Ettema, et al., 2000), while a more recent example is Aurora (Joh, et al., 2002), which originally was developed as a rescheduling model but which can also be used as a scheduling model.

\subsubsection{Evaluation}

Although modelling approaches and underlying theories differ, most have in common the notion that choice behaviour involves a value judgment (utility, satisfaction, attitude) used to rank the available choice alternatives in terms of preference, and some choice mechanism to translate this ranking into choice probabilities or choice (e.g., Morley, 1994; Oppewal, et al., 1994; Mansfield, 1995; Dellaert, et al., 1996, 1997b, 1998; Louviere and Timmermans, 1999; Stemerding, et al., 1999; Middelkoop, et al., 2001; Kemperman, et al., 2002; Bargeman, et al., 2002; Kozak, 2003; Lee, 2003; Arentze, et al., 2005).

The value judgment is typically considered to be a function of the attributes of the choice alternatives and distance or travel time separation only, conditional on socio- 
demographics. Although this conceptualization may be valuable and useful for many choice problems, this focus on individual choice behaviour does not take into account that the aggregation of such individual choices will lead to aggregate use patterns and perhaps congestion, which in turn may affect individual preferences.

\subsection{Choice behaviour in response to information}

Many decision problems in urban planning relate to the problem of trying to induce individuals to behave in a certain way such that some system-level objectives are satisfied. There is a substantial research in marketing that focused mainly on different ways in which firms can manage the amount of variations in demand that lead to service congestion (e.g., Fleischmann, et al., 2004). In particular, the effectiveness of different booking and flexible pricing strategies such as yield management (Desiraju and Shugan, 1999) and dynamic pricing (e.g., Kannan and Kopalle, 2001) have been investigated, for example in terms of how these strategies affect profits and to what degree they promote more equal usage of capacity-constrained services over time.

In addition to that, with the rapid adoption of web services by (potential) consumers for a variety of purposes, including pre-purchase or pre-trip information search or online booking or shopping, increasing attention has been paid to the role of information on individual's behaviour (Alba, et al., 1997). Travel demand management is one such example in which planning authorities are trying to reduce congestion in transportation networks by providing travel information. It may be expected that with the new available technology other forms of environmental control will be implemented.

There is a substantial literature in transportation on the use of information to influence the behaviour of travellers' route choice, mode choice, departure time choice, destination choice, etc. By providing (personalized) travel information (e.g., through the Internet or through location-based services), individual travellers are triggered to depart at a certain time, use a particular route, etc. New technologies such as web services, location-based services and personal travel assistants allow individual travellers to access such travel information to become informed about the state of the system, reducing uncertainty. The opportunities for individuals to collect information and receive advice regarding an activity schedule that best meets a set of individual criteria is increasing. Likewise, using such technologies, information providers can reach their (potential) customers, so as to influence their choices to optimize the use of the available infrastructure.

\subsubsection{Information use and provision}

To assess the impact of travel information on individual's decision, many studies have focused on the effect of advanced travel information service (ATIS) on travel choice (e.g., Emmerink, et al., 1995; Arentze, et al., 2004; Chen and Mahmassani, 2004). As Chorus, et al. (2004) argued, these studies can be categorised into two streams, those that describe travel choices given travel information (e.g., Ben-Akiva, et al., 1991; Khattak, et al., 1993; Yim and Khattak, 2002) and those that describe the process of information acquisition using ATIS (e.g., Polak and Jones, 1993; Polydoropoulou and Ben-Akiva, 1998; Hato, et al., 1999; Kenyon and Lyons, 2003). Most of this literature concerns the 
idea that information provision will reduce uncertainty and expose possible hidden alternatives (Arentze, et al., 2004).

Taking into account the stochastic and non-stationary nature of urban systems, the management of the resulting uncertainties is central to an individual's decision problem. Acknowledging this, many studies have addressed the impact of information and used expected utility to model choice behaviour under conditions of uncertainty. The concept of expected utility is important as it captures the uncertainty about the state of the environment at the time when an individual wishes to make a choice. One method for deriving expected utilities is a so-called Bayesian belief network or decision network (e.g., Arentze and Timmermans, 2004a, 2004b).

Another stream of models has focused on strategies of providing recommendations or guidance from the viewpoint of the whole system, and is typically concerned with optimizing a single objective function (i.e., the total delay in route choice). Most of these models are based on the consistency between the predicted and observed aggregate activity-travel patterns by means of an iteration scheme (e.g., Ben-Akiva, et al., 2001; Barfield and Dingus, 1998; Mahmassani and Jayakrishnan, 1991; Arnott, et al., 1991; Adler and Blue, 1998): if the users' reaction to the information is known from experiments, the resulting transportation situation can be calculated, yielding improved choice recommendations, etc. Given this iteration scheme converges, it would facilitate optimal recommendations and reliable forecasts about the transportation network, anticipating the travellers' reactions. That is, if the reaction of users is already taken into account in advance when generating the recommendation, the state that emerges is likely to be better. Within this framework, the information about congestion is part of the environmental attributes, which are taken as given in the individuals' response model.

\subsubsection{Evaluation}

Although this conceptualization may be valuable and useful for many choice problems, this focus on the attributes of choice alternatives and information about travel time may be highly non-stationary in the sense that often the utility that people derive from visiting a particular location also depends on the choice behaviour of other individuals. Moreover, the basis that information providers use to make recommendations may have a time-lag effect on the forecasts of the individuals' reactions to the recommendations, which may invalidate the recommendation.

From a behavioural perspective, however, the problem may be more complicated. Both individuals and information providers may act strategically. Individuals will choose particular choice options taking into account their expectations of the behaviour of others such as to maximize their own utility and avoid congestion. Information providers will make similar strategic decisions in light of their expectations of individuals' reactions to the information they provide, in order to induce individuals to behave in a certain way such that some system-level objectives are satisfied. When travel information is provided, individuals will make assumptions if and how other individuals will adjust their current behaviour. In light of the formulated problem, it is important to understand the interactions between the different decision makers in order to minimize congestion and to optimize the usage of the facilities in the city. 


\subsection{Choice behaviour in response to other individuals' behaviour}

The above brief overview of individual choice modelling suggests that considerable progress has been made in developing such models. Yet, there is still lack of attention to improve these models, especially with respect to the interaction and interdependencies between individuals. Such interdependence can be viewed along two lines: intrahousehold and inter-household within a social-spatial network.

\subsubsection{Interdependence within multi-person households (intra-household)}

First, such interdependence concerns decisions within multi-person households related to task allocation, allocation of resources and the decision to become involved in joint activities. Some authors have recently started to address this issue (e.g., Gliebe and Koppelman, 2002; Zhang, et al., 2002; Borgers, et al., 2002). This intra-household interdependence was generally approached by developing measures of utility for individual household members and some weight, which reflects the relative influence of individual members on the joint decision.

\subsubsection{Interdependence within in the social-spatial network (inter-household)}

Secondly, the interdependence concerns the decision made within the social-spatial network. The underlying assumption is that a given individual's choice for a particular alternative is dependent on the overall share of decision-makers that choose the same alternative (e.g., Aoki, 1995; Brock and Durlauf, 2001; Blume and Durlauf, 2002). Such dependence can be represented in two basic feedback effects: positive or negative.

Some studies expanded discrete choice models with a positive feedback in terms of "copying" behaviour that is based on the conformity pressure in the social-spatial network to model the choice of transport mode (Dugundji and Gulyas, 2003; Dugundji and Walker, 2005). In their model, individuals are grouped into geographic neighbourhoods or into socioeconomic segments. They distinguish two types of interdependent sources: the direct choice effect of the reference group and the unobserved variable influence faced by the same group. The individual is influenced by the average choice behaviour of his reference group and the influence by other groups is assumed to be negligible. Moreover the individual's own choice was excluded when computing the market share of the reference group. The systematic part of the utility function as described in equation (2.1) that an individual uses to make a choice is modified as a linear-in-parameter, first-order function of the share of the consumers in a given individuals' group choosing that alternative. In particular:

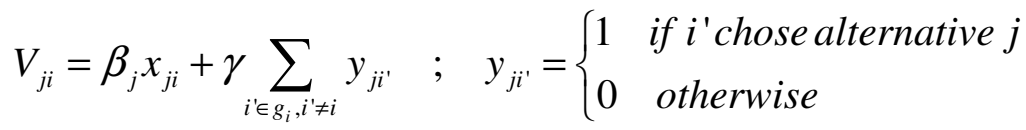

where $i$ is an index for individuals and $g$ is a group index. As we can see from the equation the choice probability of an alternative is proportional to the proportion of the number of others in the group that choose that alternative. Apparently, the type of choice 
that an individual makes does not explicitly or implicitly represent the network structure nor affects his reference group position in the social-spatial network. The feedback effect in this type of model is not complete, since the standards that are used to categorize individuals to different reference groups in the social-spatial network have no direct links with the individual's choice. For example, whether a household has to choose between carpool or public transport, or between a multi-modal trip or a uni-modal trip, does not essentially change the exogenously determined fact to which reference group that household belongs.

Although their model puts forward an interesting mechanism of modelling interaction and interdependence between individuals, especially in the easy way of using aggregate data, their approach may have a few weaknesses. First, their model is fundamentally grounded in a positive feedback effect. It is difficult from its theoretical underpinnings, although not impossible, to extend the model to include negative feedback effects. Second, in practice, it can be difficult to reveal the exact details of the relevant network(s) of the reference group that influence the choice of each individual. The actual reference groups for a given individual may not even be included in the data sample. Third, and most importantly, the particular choice problems that they deal with are by their very nature different from the choice problems that do affect the "sorting" of households into reference groups, such as residential location choice. Such choices endogenously impact the household's reference position in a spatial network and potentially also within a social network (e.g., in moving to a new neighbourhood).

In general then, we argue that the interaction and interdependence between individual decision-making and aggregate activity-travel patterns involves more complex feedback mechanisms, which are not restricted to which social or spatial group the individual belongs to. As we introduced in the beginning of the thesis, the individual and household activity-travel decisions lead to aggregate, macro-level, activity-travel patterns, which evolve over time, during the day. An individual, micro-level, decision thus has to be made in light of uncertain and unknown aggregate, time-dependent, activity-travel patterns. Many examples in the field of transportation, tourism and leisure come to one's mind as an illustration of this principle. For example, when going to a restaurant, dependent on one's preferences for crowdedness or quietness, the timing of the visit will not only depend on the quality of the food and the ambience of the restaurant, but also on an individual's expectations about the choice of other restaurant visitors. Similarly, the best time to visit a museum or theatre, choice permitting, is likely influenced not only by individual value judgments related to the attributes of the choice alternative and the value of time, but also by expected crowdedness. In order to incorporate this type of feedback mechanism to represent the impact of such aggregate activity-travel patterns that emerge from the multitude of individual activity-travel decisions, we investigated the relevant literature on interactive decision making.

\subsubsection{Game theory and interactive decision making}

Non-cooperative game-theory may be a useful theory to study such interactive decision making processes under conditions of uncertainty. This theory, which emanated from studies of games such as chess or poker, assumes that players have to think ahead and devise a strategy based on expected countermoves of the other player(s). Such strategic interaction characterizes many economic situations, and this theory has therefore proved 
to be very useful in various economic analyses. It may also be useful in spatial choice analysis. The advantage of this approach is that it is based on some primitive assumptions about payoffs and strategies, and that it uses the concept of equilibrium to predict the outcome of strategic interaction (e.g., Rasmusen, 1990; Scott Bierman and Fernandez, 1998).

Game theory is an instrument designed to help understanding the phenomena that we observe when decision makers interact and explaining the strategic reasoning behind it. Game-theoretical models are highly abstract representations of real-life situations, which allow them to be used to study a wide range of phenomena. It uses mathematics to express formally underlying concepts. This makes it easy to precisely define concepts, to verify the consistency of ideas, and to explore the implications of assumptions, although, most game theoretical ideas are not inherently mathematical. The basic assumptions that underlie the theory are that decision makers pursue well-defined exogenous objectives (they are rational) and take into account their knowledge or expectations of other decision makers' behaviour (they reason strategically). The basic ingredients of game theory are:

- There are two or more decision makers, called players.

- Each player has a choice of two or more ways of acting, called strategies. The outcome of the interaction depends on the strategic choice of all players. The essential feature of the social interaction is that each decision maker has only partial control over the outcome.

- The players have well-defined preferences for the possible outcomes, so that numerical payoffs reflecting preference can be assigned to all players for all outcomes. One important structural property is the way in which the players' payoff functions are related to one another.

- The objective is to determine, through formal reasoning alone, what strategies the players ought to choose in order to pursue their own interests rationally and what outcomes will result if they do so.

Game theory has long been used to analyze individuals' economic behaviour and provides a deep and illuminating insight interpretation. Since spatial choice behaviour may be viewed as a subset of general economic behaviour, game theory can provide a powerful analytical tool to study the addressed interaction problem.

Another aspect of the research problem addressed in this thesis is decision-making under uncertainty. In this context, it is important to distinguish between uncertainty due to uncertain acts of nature and uncertain acts of other individuals. In traditional decision theory, each individual is making a choice among the alternatives against nature, but it does not explicitly or even implicitly explain that the uncertainty partly arises from the choice behaviour of other individuals. That is, they involve in a situation called strategic choice, where other individuals' choices become a state variable that is endogenously determined.

Uncertainty in strategic situations means a lack of information, and may take on many different forms. The individuals may lack full information about the other individuals' (or even their own) payoff functions, about the physical or the social resources, about the strategies available to other individuals (or even to them themselves), about the amount of information the other players have about various aspects of the situation, and so on. But by suitable modelling, all forms of incomplete information can 
be reduced to the case where individuals have less than full information about each other's payoff function.

\subsubsection{Game theory and discrete choice models}

We could decompose the described problem into two layers: at the lower level - decision among individuals as a distribution problem; at the high level - decision between the collective individual and information provider as a control problem. The combined distribution-control problem could be interpreted as the following two games being played: the first game - the distribution problem is an n-player non-cooperation game among individuals, in which individuals maximize their own individual utilities; the second one - the control problem is a game between the information provider and the collective individuals, in which the information provider maximizes an overall utility by offering recommendations, while taking individuals' response into account.

A particularly novel approach to distribution-control interactions (analogy supplydemand interactions) in the literature is the studies that combine ideas from game theory and discrete choice theory. For example, Chen (1998) developed a game theoretic model to find a mutually consistent dynamic system-optimal signal setting and dynamic useroptimal traffic flow. Brewer and Hensher (2000), and Rose and Hensher (2004) developed what they term "interactive agency choice experiments" between employer and employee to study adoption of participation in distributed work and group decisions making. Bajari and Kahn (2001), and Bayer, et al. (2004) presented equilibrium models describing how residential location choices of households aggregate to form a housing market equilibrium.

In general, these approaches begin by relating discrete data on individual's decisions to game-theoretic models of individual's actions, information, payoffs, and strategies. Then, the axiom of preference used in traditional discrete choice models is replaced with an equilibrium solution concept that identifies individuals' most preferred strategies. A stochastic specification added to individual's payoffs, together with the games solution concept, permits the calculation of the probabilities of the game's outcomes and thereby to construct a likelihood function for observed data. One thus can develop statistical response models from game-theoretic models, providing a straightforward way of gaining insights into the behaviour of individuals in real life situations that involve interactive decision making.

\subsubsection{Evaluation}

This brief review indicates that some recently developed models of individual behaviour in response to other individuals' behaviour do capture some specific interactions and interdependencies among individuals such as joint choice within households and copying behaviour within the same social-geographic group. Strategic interaction, however, is not addressed in these models and therefore needs more attention because strategic choice behaviour represents an important class of spatial and non-spatial choice problems, especially under conditions of uncertainty and congestion.

Game theory is a theory of strategic interaction. That is to say, it is a theory of rational behaviour in social situations in which each individual has to choose his move on 
the basis of what he thinks the other individuals' countermoves are likely to be. It refers to how individuals can assess outcomes and choose alternatives that will yield preferred outcomes, given the choices of others. The objective is to determine, through formal reasoning alone, what alternatives the individual ought to choose in order to pursue his own interests rationally and what outcomes will result if the entire set of individuals involved do the same.

There are examples of an approach which combines game theory and discrete choice theory to deal with specific problems in various domains. Most of these formulate a discrete choice model with endogenous interactions, in which the choices of other individuals are explanatory variables in the equation describing the choice behaviour of a given individual. These models have proven to be successful in modelling interactive decisions, and can therefore potentially be used as a reference for our purpose, as the problem described in this thesis can be formulated as a special case of games.

\subsection{Conclusion and discussion}

This chapter briefly reviewed the existing literature on spatial choice with interactions. The review has identified a number of issues that are important to the approach that will be adopted in this thesis and that can also be used to position this thesis against existing work. First, we have argued that the literature on individual spatial choice modelling has been successful in numerous fields but lacks feedback mechanisms that count for the effect of aggregated individuals' choices, and thus that there seems a need to explore the possibility of developing choice models of strategic behaviour that try to capture interdependencies in individuals' choices. Secondly, we have shown that as information is used as one of the instruments for controlling congestion, more research is required on how to model interactions between an information provider and individual users. Thirdly, to the extent that such modelling attempts should be based on interactive decision making, game theory was introduced and a few applications were briefly mentioned. By combining what we see as the best features of these two lines of research, our goal in the remainder of thesis is to develop a general and flexible framework useful for modelling strategic behaviour under conditions of uncertainty in anticipation of congestion. 


\section{Chapter 3}

\section{Theory}

\subsection{Introduction}

In the previous chapter the literature on spatial choice models was briefly reviewed and it was argued that none of the existing models of spatial choice behaviour take into account that the attributes of the choice alternatives and travel time may be highly non-stationary and that the utility individuals derive from visiting a particular location may also depend on the choice behaviour of other individuals, especially in congested environments. Therefore, the review was extended to incorporate non-cooperate game theory and it was concluded that the combined interaction between different decision makers could be studied in terms of games.

In this chapter, we will introduce a strategic choice model derived from discrete choice theory integrated with a game-theoretic mechanism. We will argue that gametheoretic models are potentially valuable in modelling strategic behaviour in anticipation of congestion.

The chapter starts with formulating the distribution problem between individuals as an $N$-player $J$-option game. Then, we will discuss how such an approach can be integrated with well-known discrete choice models to model individuals' strategic behaviour in anticipation of congested situations. This is the main focus of the theoretical contribution of this thesis. On the basis of this, we differentiate between three levels of expectation of others: (1) expectation with complete information in ideal situations - Nash equilibrium, 
(2) expectation with incomplete information in repeated situations - quantal response equilibrium, and (3) expectation with incomplete information in one-shot situations noisy introspection. Some critical issues in the application of such models are discussed.

Next, the distribution-control problem between the information provider and the collective individual is formulated as a 2-player 2-stage game. The individual's strategic choice model is extended to incorporate choice behaviour in response to the information. It continues with various objectives that different kinds of information providers may have.

\subsection{Models of strategic choice behaviour based on interaction and interdependencies between individuals}

The general problem area that we address concerns the problem of the timing of an activity. Examples would be the optimal time to use a certain capacity-constraint service, such as the time going to a restaurant, visit a museum, visit a city, etc. To study how individuals' timing decisions or choices are influenced by similar strategic considerations of other individuals, a game-theoretic concept is adopted. The quintessence of this conceptual framework is the assumption that in light of uncertainty and interdependent situations individuals think ahead and develop a strategy based on expected timing decisions of other individuals. Individuals are assumed to have expectations of the probability that other individuals will choose to use the service during each time slot. Based on these expectations, the expected utility of each time slot can be derived, and a choice can be made. Thus, the model defines the interaction between an individual and the aggregate activity-patterns of all other individuals. In this section, we first give a game formulation of the problem that we described. Then, we integrate this with a discrete choice approach to model an individual's utility function for different access times when taking into account other individuals' anticipated access time choices. Next, we model the equilibrium that arises when such access time choices are made simultaneously. Finally, various levels of rational behaviour as well as the application of the model will be discussed.

\subsubsection{N-player $J$-option game formulation}

Game theory is a theory about strategic interaction. That is to say, it is a theory of rational behaviour in social situations in which each individual has to make his choice on the basis of what he thinks the other individuals' choices are likely to be. Before we outline in detail our specific game formulation, we briefly discuss some general definitions and key principles of game theory that will be used in this section.

In normal (or strategic) games, the players move (choose their actions) simultaneously and independently. The players are individuals who make decisions. Each player's goal is to maximize his utility by choosing the appropriate actions. A strategy is a detailed set of plans for choosing the action. This means that a strategy for a player must specify the choice to make at each of the possible situations. The knowledge at a particular time about the possible values of different variables in the game constitutes a player's information set. A pure strategy $\left(s_{i}\right)$ maps each of a player's possible information sets $\left(w_{i}\right)$ to one action $\left(s_{i}: w_{i} \rightarrow a_{i}\right)$. A mixed strategy maps each of a 
player's possible information sets to a probability distribution across actions $\left(s_{i}: w_{i} \rightarrow p\left(a_{i}\right)\right.$, where $p \geq 0$ and $\left.\int_{A_{i}} p\left(a_{i}\right) d a_{i}=1\right)$. In contrast to pure strategies, mixed strategies are strategies that involve random draws. Players randomize intentionally and the probability mixtures they use are not arbitrary. A mixed strategy is a probability distribution over a player's (pure) strategies. For example, in penalty shooting, the goal shooter typically does not expect the goalkeeper to jump to the right corner for sure, but he regards the goalkeeper's behaviour as a mix between the pure strategies "jump to the right" and "jump to the left".

Central in game theory is the concept of strategic equilibrium. An equilibrium is a profile of strategies that given a profile of beliefs and equilibrium behaviour cannot be unilaterally improved, implying that the beliefs are consistent with actual courses of action prescribed by the equilibrium strategies. The Nash equilibrium is a profile of strategies of "best responses" to one another, or a profile of strategies that involve "optimal reactions" to "optimal reactions". The Nash equilibrium is the pure form of the basic concept of strategic equilibrium; as such, it is useful mainly in normal games.

Another central concept is expected utility. It can best be considered, based on Von Neumann-Morgenstern interpretation (1944), as an axiomatic extension of the conventional concept of utility to uncertain payoffs. A player possesses a Von NeumannMorgenstern utility function if he ranks uncertain payoffs according to their expected utility value of the outcomes. An outcome of a game is a certain payoff allocation on the basis of a profile of strategies.

As described in chapter 2, the first game considered is played at the disaggregate level, which is formulated as an $n$-player non-cooperative game among individuals. We use individuals choosing a time slot to conduct an activity as an example of context to explain the meaning of this statement. To be more rigorous, it is supposed that there are $N$ players in the game and they are numbered from 1 to $N$. Moreover, there are $J$ options that available to the players. In this case, we assume that each player's information is imperfect, certain, symmetric and incomplete. Imperfect means that players do not know other players' behaviour (i.e., their activity timing choice). Certain means that they have some idea of what other players might do or what possible moves they could make. Symmetric means that no player has more information than any other players. Incomplete means that each player has some private information about his own payoff that is unobserved by other players and the modeller.

To solve the uncertainty in the game, it is suggested that the uncertainty could be due to the existence of a small amount of uncertainty about the opponent's payoffs. In game theory, the so-called Harsanyi transformation (1967, 1968a, 1968b) treats players who have different payoffs as being of distinct types. It proposed that such games be modelled by having "Nature" move first and "choose" each player's type. Players start out knowing their own type but not that of their competitors. Players share a common belief as to how "Nature" makes its probabilistic choice. The resulting new game structure is called a Bayesian game. Following the general concept of a Bayesian Nash equilibrium, a static Bayesian game can be described by its strategic form, which consists of five lists, all of which involve common knowledge. By common knowledge, we refer to information that is known to all players, each player knows that all of them know, each of them knows that all of them know that all of them know, and so forth. In our case, we define the five lists as below (see Figure 3-1 for a summary): 


\section{- Players}

- Individuals

\section{- Information}

- Imperfect, certain, symmetric, incomplete

\section{- Strategy and moves}

- Activity timing choice

- Independent, simultaneous move

\section{- Payoffs}

- The utilities of player's timing choice

\section{- Bayesian Nash Equilibrium}

\section{Figure 3-1 Game 1 formulation}

- First is the list of players, i.e., the individuals that participate in the activity.

- Second is the list of moves for each player. A list of moves, one for each player, is a move profile and will be enclosed in curly brackets \{\} . In this context, a player's move is the choice of activity start time. Each individual chooses one option out of his/her feasible options independently. When an individual makes a choice, he/she does not know which options other individuals select, as if they move simultaneously.

- Third is a list of possible types for each player. The payoff-relevant private information of a player, like the numerical level of a certain parameter in this Von Neumann-Morgenstern utility function, is called his type. Every player knows his/her own type but may not know the type of the other players. The other players are denoted by the index $-i$. A list of types, one for each player, is a type profile and is enclosed within square brackets []. A player often does not know the values that others place on the possible outcomes. In other words, a player may not know the other players' preferences that lead to their payoffs and as a consequence to their moves. We use an error term to describe the uncertainty of the perceived payoffs and transfer it to the players' type.

- The players have a common prior belief about the probability of each possible type of profile. The probability that the player's profile is $\left[t_{1}, \ldots, t_{N}\right]$ is denoted $P\left[t_{1}, \ldots, t_{N}\right]$. These probabilities comprise the fourth list.

- Every move profile $\left\{s_{1}, \ldots, s_{N}\right\}$ and type profile $\left[t_{1}, \ldots, t_{N}\right]$ result in a payoff for each player. Player $i$ 's payoff will be denoted in general as $U_{i}\left(s_{1}, \ldots, s_{N}, t_{1}, \ldots, t_{N}\right)$. The payoffs make up the fifth list. The payoff function is the overall utility for this player's choice. The game tree (Figure 3-2) gives the extensive form of an $n$ player game for a start time choice problem. In this form, the players choose their start times as if they happen in sequence, but actually when one player makes a decision, he does not know the choices selected by the other players. The dashed line that connects the nodes at each player's level indicates that those nodes are 

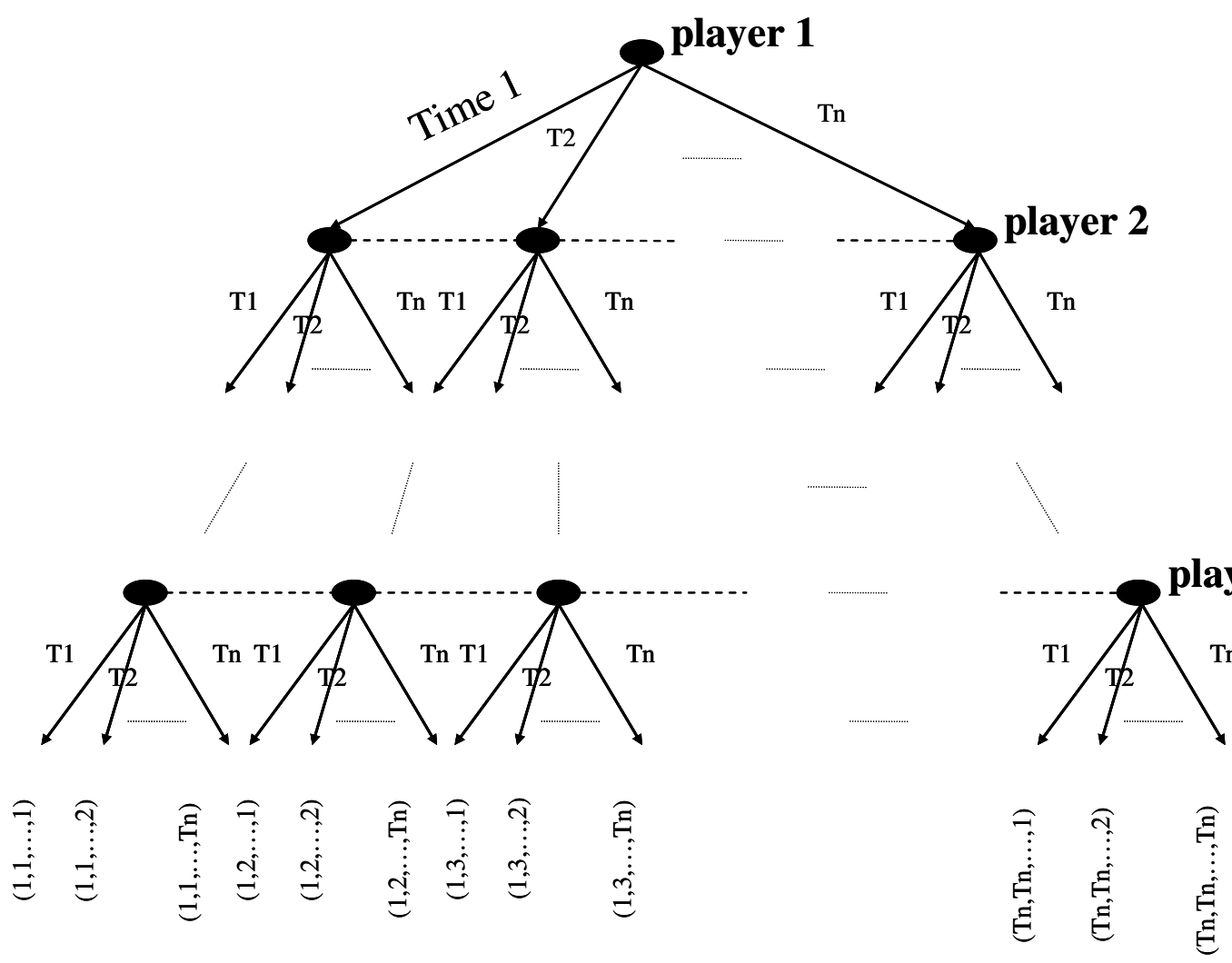

Figure 3-2 Game 1 extensive form

indistinguishable. Each leaf at the end of the tree represents a possible outcome of the game.

In general, player $i$ 's move may depend on his type. Hence, a strategy for player $i$ consists of a move as a function of player's type and will be denoted as $S_{i}\left(t_{i}\right)$. A list of strategies, one for each player, is a strategy profile and will be enclosed within curly brackets. Since the players' types are taken as randomly selected by "Nature", the outcome of a strategy profile will be uncertain. We assume the players are expected utility maximizers and simultaneously choose among strategies on the basis of conditional expected utility. The conditional expected utility (CEU) of player $i$ of type $t_{i}$ when the players adopt the strategy profile $S=\left\{S_{1}\left(t_{1}\right), \ldots, S_{N}\left(t_{N}\right)\right\}$ is:

$E U_{i}\left(S, t_{i}\right)=\sum_{t_{-i}} U_{i}\left(S_{1}\left(t_{1}\right), \ldots, S_{i}\left(t_{i}\right), \ldots, S_{N}\left(t_{N}\right), t_{1}, \ldots, t_{i}, \ldots, t_{N}\right) \cdot P_{i}\left[t_{-i} \mid t_{i}\right]$

where the summation is over all possible type profiles of the other players. The definition of a normal form non-cooperation game with a Bayesian Nash equilibrium is (e.g., Scott Bierman and Fernandez, 1998): 
A strategy profile $S^{*}=\left\{S_{1}^{*}\left(t_{1}\right), \ldots, S_{N}^{*}\left(t_{N}\right)\right\}$ is a Bayesian Nash equilibrium of a static Bayesian game if and only if for every player $i$, every type $t_{i}$ of player $i$, and every alternative strategy of player $i$, say $S_{i}\left(t_{i}\right)$ :

$$
E U_{i}\left(S^{*}, t_{i}\right) \geq E U_{i}\left(S_{1}^{*}\left(t_{1}\right), \ldots, S_{i}\left(t_{i}\right), \ldots, S_{N}^{*}\left(t_{N}\right), t_{i}\right) .
$$

That is, the player's strategy is a best response to the strategies of the other players, whatever the player's type.

This implies that each player's strategy $S_{i}^{*}$ solves: $\max _{s_{i} \subset S_{i}} U_{i}\left(S_{1}^{*}, \ldots, S_{i}, \ldots, S_{N}^{*}\right)$. If no single player can improve his/her payoff function by unilaterally changing his/her choice, then it is essentially a Nash equilibrium, in which players maximize their own individual utilities. Obviously, different assumptions about the utility functions determine the outcome of the equilibrium.

\subsubsection{Strategic discrete choice model}

Assume a set of $N=\{1, \ldots, i, \ldots, N\}$ individuals, and for each individual $i \in N$ a strategy set $S_{i}=s_{i 1}, s_{i 2,} \ldots, s_{i J}$ of $J_{i}$ pure strategic choices. $s=\left(s_{1}, \ldots, s_{N}\right) \in S$ is a strategy combination, and it consists of $n$ strategies, one for each individual. $s_{-i}=\left(s_{1}, \ldots, s_{i-1}, s_{i+1}, \ldots, s_{N}\right)$ is a strategy combination of all individuals except $i$. For each $i \in N$, there is a payoff function, $u_{i}: S \rightarrow \Re$, where $S=\prod_{i \in N} S_{i}$ is the Cartesian product of the individual strategy spaces, representing choices of actions. This payoff function represents the utility that an individual derives from selecting a particular strategic response to the strategies that other individuals might choose.

Put into a discrete choice framework, individual $i$ 's payoff for strategy $j$ consists of three components: (1) a choice alternative-specific component, $U^{\text {exogenous }}$, which expresses the exogenous attractiveness for a given alternative, (2) an interaction component, $U^{\text {endogenous }}$, which captures the expected impact of other individuals' choice behaviour, and (3) an idiosyncratic error term, $\varepsilon$, treated as an individual and alternativespecific random variable whose distribution is common knowledge among all individuals, but whose exact value is private information to the individual. Thus,

$U_{i j}=U_{j}^{\text {exogenous }}+U_{j}^{\text {endogenous }}+\varepsilon_{i j}$

\subsubsection{Specification of the exogenous component}

The first term in equation (3.1) represents the traditional exogenous attributes of the choice alternatives that affect payoffs. This term can be formulated, for example, as: 


$$
U_{j}^{\text {exogenous }}=\beta X_{j}=\beta_{1} X_{j}^{\text {preference }}+\beta_{2} X_{j}^{\text {cost }}
$$

The preference accounts for the variation in activity utility over time and space, and reflects the preference to become involved in the activity and the preferred time to conduct the activity. The cost includes time pressure and travel cost, which could, depending on the specific application, be further expanded to account for location accessibility, travel time, route benefit, entrance fee, etc. (see also Gärling, et al., 1998).

It might be useful to characterize the decision maker by another vector of attributes, if the preferences or tastes of different members of the population vary systematically with some known socioeconomic attributes, such as income, car ownership, household size, age, occupation, and gender. For example, it is not unusual to define as an attribute cost divided by income, reflecting the a priori belief that the importance of cost declines as the inverse of an individual's income.

Similar to conventional discrete choice models, linearity in parameters is not as restrictive as it may seem at first glance. It allows for any function of the attributes so that polynomial, piecewise linear, logarithmic, exponential, and other transformations of the attributes are valid for inclusion as elements of $\boldsymbol{X}$.

\subsubsection{Specification of the endogenous component}

The next term in equation (3.1) captures the attractiveness of a choice alternative as a function of the behaviour of other individuals. It consists of two parts: the expected number of individuals that choose the same alternative $(n)$ and the variable impact of these individuals. Thus,

$U^{\text {endogenous }}=h\left(f_{j}(n), n\right)$

where $f_{j}(n)$ allows a flexible specification of the effect of inter-individual interaction on utility, depending on the characteristics of the context. For instance, if an individual's preferred activity start time is also the preferred start time of many other individuals, the concentration of individuals at a particular point in time and space will, in some cases, adversely affect the individual's utility due to crowdedness (congestion) reflected in increased cost of queuing and reduced service quality, while in some other cases it will favourably influence the individual's utility due to economies of temporal agglomeration (e.g., more efficient communication in work or school).

We could use an identical linear utility function to imply that the marginal effects of additional other individuals choosing the same alternative is additively constant across different alternatives. We also could use more flexible nonlinear functional forms that allow the impact to increase or decrease at a varying rather than constant rate. Furthermore, we could allow a more refined impact of other individuals. For example, a two-dimensional matrix of impact effects for alternative pairs will represent different interaction. 


\subsubsection{Specification of the disturbance}

Finally, a useful interpretation of the third term in equation (3.1) is that it captures unobserved heterogeneity in preference across otherwise identical individuals. An alternative interpretation is that individuals calculate expected payoffs correctly but have an additive payoff disturbance associated with each available pure strategy, which is endogenous and relevant to the individual's decision making. This disturbance may be due to either error (such as cognitive limitations, distractions and perception biases that lead to non-optimal decisions) or unobserved utility that make rational behaviour look noisy to an outside observer - the modeller. Without this term, there is no private information, and then every player has perfect and complete information about each other, while the modeller can perfectly observe. In actual applications the term will be a composite of a great number of unobserved effects, each of which contributes in some way to the distribution. The terms, $\varepsilon_{i j}$, may also be some function but it is random from the observational perspective of the modeller in this case.

From a modeller's point of view, the utilities are treated as random due to observational deficiencies. There are four distinctive sources of randomness identified by Manski (1973) in traditional discrete choice models resulting from: (1) unobserved attributes, (2) unobserved taste variations, (3) measurement errors and imperfect information, and (4) instrumental or proxy variables. In addition to these, in strategic situations, there is one additional possible source when individuals' utilities are sensitive to decisions of others, which is the error in the expectations. Players are mutually uncertain of each other's incentives, although they do know the general distribution of those incentives. Due to these uncertainties, individuals may differ in terms of their experiences that cause their preferences to differ, and make errors in their adjustment of strategies given their expectations. To overcome this complication, we assume that individuals are rational, strategic actors who might make mistakes, but who can develop expectations and are aware of the possible mistakes of others.

\subsubsection{Equilibrium concept: consistency condition}

The main difference with the decision-making context in a normal situation is that, in a strategic equilibrium model, the exogenously given incentives are partly replaced by endogenously determined expected payoffs. The added complication, in contrast to traditional individual choice models, is that the choice probabilities of the players become an important interactive component, since they are simultaneously determined in equilibrium. Thus, for a given equilibrium concept that individuals may use to determine an anticipated interaction, we expect that individuals take into account other individuals' anticipated choices when making their own choice. In other words, individuals' anticipations of other individuals' choices affect their own preference for different alternatives. Furthermore, when anticipating other individuals' choices, each individual needs to take into account not only the other individuals' preferences for the exogenous attractiveness of the alternative, but also how these individuals respond to the potential endogenous impact and adjust their choices accordingly. 


\subsubsection{Bayesian Nash equilibrium}

Following traditional game theory (Fudenberg and Tirole 1991), the Bayesian Nash equilibrium (BNE) constitutes the optimal response that maximizes an individual's expected payoff, given his conjectures about other individuals' strategies. That is, in equilibrium, individuals choose strategies $\left(s_{1}^{*}\left(\varepsilon_{1}\right), \ldots s_{i}^{*}\left(\varepsilon_{i}\right), \ldots, s_{n}^{*}\left(\varepsilon_{n}\right)\right)$ such that, for each individual $i$ and each of its possible types $\left(\varepsilon_{i}\right)$, strategy $s_{i}^{*}\left(\varepsilon_{i}\right)$ maximizes the expected payoff across alternatives, given that individuals' type follow some prior distribution. Each individual's pure-strategy Bayesian Nash equilibrium choice is formulated as:

$s_{i}^{*}\left(\varepsilon_{i}\right) \in \arg \max _{S_{i}} \int_{\varepsilon_{-i}} U_{i}\left(s_{i}\left(\varepsilon_{i}\right), s_{-i}\left(\varepsilon_{-i}\right),\left(\varepsilon_{i}, \varepsilon_{-i}\right)\right) f\left(\varepsilon_{-i}\right) d \varepsilon_{-i}$

where $U_{i}\left(s_{i}\left(\varepsilon_{i}\right), s_{-i}\left(\varepsilon_{-i}\right)\right.$ denotes the utility to individual $i$ of choosing choice strategy $s_{i}($.$) , conditional on the individual's specific term \varepsilon_{i}$, when the other individuals follow strategy $s_{-i}($.$) , and across the set of specific preferences \varepsilon_{-i}$ for all individuals, excluding individual $i$. This function expresses the fact that the expected payoffs are calculated from the equilibrium distribution of joint strategies. Together with the prior distribution for his type, a strategy profile of individual $i$ determines an individual distribution over the possible compositions of types with actions.

$p\left(\varepsilon_{-i} \mid \varepsilon_{i}\right)$ denotes individual $i$ 's conditional probability about other individual's types $\varepsilon_{-i}=\left(\varepsilon_{1}, \ldots, \varepsilon_{i-1}, \varepsilon_{i+1}, \ldots, \varepsilon_{n}\right)$ given his type $\varepsilon_{i}$. Based on the common knowledge that the strategies of others are a function of their type, individual $i$ could use his beliefs $p\left(\varepsilon_{-i} \mid \varepsilon_{i}\right)$ to compute the expected utility for each choice and thus find his optimal response. This probability is calculated by integrating across the distribution of other individuals' error distributions.

An individual's strategy would then be a function that specifies a feasible action for each of the individual's possible types. Alternatively, an individual's possible types correspond to different players, one of whom will be randomly selected to be active in the game. The point is that each individual's optimal decisions will maximize his conditional expected payoff given his actual type, and any expected value that is not conditional on such type information is not significant.

As summarized in the Bayesian game model, the game must be analyzed from the perspective of someone who only knows the information common to all individuals. As an outside observer/modeller, we do not have any knowledge of any individual's actual type, so that we could appreciate the uncertainty of the other individuals who do not know it as well. The actual type of each individual, being private information, is treated as an unknown quantity or random variable in our analysis. Thus, a Nash equilibrium of a Bayesian game specifies a feasible action for every possible type of every individual, such that the specified action for each type of each individual maximizes his conditional expected payoff, given his type, given his beliefs about the other individuals' type, and given the type-contingent actions of the other individuals according to this equilibrium.

As their best responding strategies, however, depend on the other individuals' actions, which further depend on the individuals' actual types, an individual must see 
himself through his opponents' eyes and plan an optimal reaction against the possible strategies of his opponents for each potential type of his own. Thus, a strategy in a Bayesian game of incomplete information must map each possible type of each individual into a plan of actions. Then, since the other individuals' types are unknown, each individual forms a best response against the expected strategy of each opponent, where he averages over the (well-specified) reactions of all possible types of opponents, using his prior beliefs about the possible type distribution. Such a profile of type-dependent strategies, which cannot be improved unilaterally, forms a Bayesian Nash equilibrium. Basically, a Bayesian Nash equilibrium is thus a Nash equilibrium 'at the interim stage' where each individual selects a best response against the average best responses of the competing individuals. Consequently, if there is only one type for all individuals as common knowledge, then a Bayesian Nash equilibrium is a purified Nash equilibrium with complete information.

Due to the symmetry of individuals' error terms, which are draws from the same distribution, each individual will have the same conjecture about any other individual $k$ 's optimal strategy. Then, individual $i$ 's belief of the prior probability that $k$ will choose strategy $j$ equals:

$$
\begin{aligned}
p_{i j} & =\operatorname{Pr}\left(s_{k j}(.)=1\right)=\operatorname{Pr}\left(E_{k}\left[U_{k j}\left(\varepsilon_{k j}\right)\right] \geq E_{k}\left[U_{k j^{\prime}}\left(\varepsilon_{k j^{\prime}}\right)\right]\right) \\
& =\operatorname{Pr}\left(E_{k}\left[\bar{U}_{j}\left(s_{-k}\right)\right]+\varepsilon_{k j} \geq E_{k}\left[\bar{U}_{j^{\prime}}\left(s_{-k}\right)\right]+\varepsilon_{k j^{\prime}}\right), \quad \forall i \neq k, \forall j \neq j^{\prime}
\end{aligned}
$$

Consequently, no individual's particular preferences affect the equilibrium. That is, individual $k$ 's belief of the prior probability that individual $i$ will choose strategy $j$ will coincide with individual $i$ 's belief of the prior probability that individual $k$ will choose strategy $j$. It implies furthermore that in equilibrium the probabilities for all individuals are identical $p_{i j}^{*}=p_{k j}^{*}=p_{j}^{*}$ as are the non-random utilities for each alternative $\bar{U}_{j}^{*}$.

The model uses the notion that one individual's choice probability is the result of integrating other individuals' discrete choice into equilibrium, and his own choice probability is continuous in other individuals' anticipation. Equilibrium consistency conditions relate to the belief probabilities that determine expected payoffs should match the decision probabilities determined by expected payoffs via some choice mechanism that made the transfer. Furthermore, the choice probabilities are an increasing but possibly non-linear function of expected-payoff difference, i.e., choice is a stochastic response. The equilibrium choice probability conjectures across all alternatives $j$ is consequently defined by the set of $J$ equations with the summation constraint of 1 , which is a fixed-point mapping following Brouwer's fixed-point theorem (e.g., Rasmusen 1989):

A continuous function mapping a compact, convex set into itself has a fixed point. If $f: B \rightarrow B$ is a continuous function defined for $x \in B \subset R^{n}$, where $B$ is compact and convex, then there exists an $x^{*}$ such that $f\left(x^{*}\right)=x^{*}$. 
This implies that, in the equilibrium, the belief probabilities that determine the expected payoffs on the right side of equation (3.5) match the decision probabilities on the left that result from applying a probabilistic choice rule to those expected payoffs.

\subsubsection{Quantal response equilibrium}

When game theory started to be used for explaining actual experimental behaviour, many researchers examined decision errors, which are not explicitly included in classical game theory. By relaxing the assumption of perfect decision making (no error) and perfect foresight (no surprises), an alternative way to arrive at the strategic equilibrium is through probabilistic choice or "quantal response" (e.g., McFadden, 1981). This concept was developed to explain why observed decisions appear to be more random as the strength of a stimulus becomes weaker (e.g., Luce, 1959). The concept of quantal response equilibrium (QRE) introduced by McKelvey and Palfrey (1995) provides a general framework for extending the probabilistic choice approach to the analysis of strategic choice. We summarize the fundamental features of the QRE below.

Let $\Sigma_{i} \equiv \Delta^{J_{i}}$ be the set of probability distributions over pure strategies $\left(S_{i}\right)$. An element $\sigma_{i} \in \Sigma_{i}$ is a mixed strategy, which is a mapping from $S_{i}$ to $\Sigma_{i}$, where $\sigma_{i}\left(s_{i}\right)$ is the probability that individual $i$ chooses pure strategy $s_{i}$. It is assumed that individual $i$ chooses a strategy with probability $p$.

Let $\Sigma=\prod_{i \in N} \Sigma_{i}$ be the set of mixed strategy profiles. Given a mixed-strategy profile $\sigma \in \Sigma$, individual $i$ 's expected payoff is $U_{i}(\sigma)=\Sigma_{s \in S} p(s) U_{i}(s)$, where $p(s)=\prod_{i \in N} \sigma_{i}\left(s_{i}\right)$ is the probability distribution over pure-strategy profiles induced by $\sigma$.

For each individual $i$ and each $j \in\left\{1, \ldots, J_{i}\right\}$, and for any $\sigma \in \Sigma, U_{i j}(\sigma)$ denotes the expected payoff for $i$ of adopting the pure strategy $s_{i j}$ when the other individuals use $\sigma_{-i}$. It is assumed that for each pure strategy $s_{i j}$, there is an additional privately observed payoff disturbance $\left(\varepsilon_{i j}\right)$, which captures error in payoff that could come from three parts: (1) own preference uncertainties, (2) others' strategies uncertainties, and (3) measurement errors. Thus, we denote $i$ 's disturbed payoff by $U_{i j}(\sigma)=\bar{U}_{i j}(\sigma)+\varepsilon_{i j}$.

For each individual $i$, his set of payoff disturbance, $\varepsilon_{i}=\left(\varepsilon_{i 1}, \ldots, \varepsilon_{i J}\right)$, has a joint distribution with several properties. First, one assumes absolute continuity (more specifically that the distribution of $\varepsilon_{i}$ can be represented by a density function $\left.f_{i}\left(\varepsilon_{i}\right)\right)$ and that the marginal densities exist for each $\varepsilon_{i j}$. Furthermore, the disturbances are assumed to be independent across individuals and strategies, and unbiased, in the sense that $\mathrm{E}\left(\varepsilon_{i}\right)=0$ for all $i$.

The assumed choice behaviour is that each individual chooses strategy $s_{i j}$ when $U_{i j}(\sigma) \geq U_{i k}(\sigma)$ for all $k=1, \ldots J$. Given this choice behaviour, $U=\left(U_{1}, \ldots, U_{n}\right)$ and $f=\left(f_{1}, \ldots, f_{n}\right)$ together induce a distribution over the actual choices of each individual. 
To be more specific, for any $U$, define $B_{i j}(U)$ to be the set of realizations of $\varepsilon_{i}$ such that strategy $s_{i j}$ has the highest disturbed expected payoff, $U_{i}$. Then,

$p_{i j}(U)=\int_{B_{i j}(U)} f(\varepsilon) d \varepsilon$

where $p_{i j}(U)$ is the induced choice probability that individual $i$ selects strategy $j . p_{i}$, which maps $U_{i}$ into $\Sigma_{i}$, is called $i$ 's structural quantal response function. Since $p(U) \in \Sigma$ and $U=U(\sigma)$ is defined for any $\sigma \in \Sigma, p \circ U(\sigma)=p(U(\sigma))$ defines a fixed point mapping from $\Sigma$ into itself.

Due to the assumption of symmetry, the choice probabilities of any one individual will affect the beliefs and hence the expected payoffs of others in the same way, since each individual has its own expected utilities over alternatives with the same payoff structure and is engaged in the same utility-maximizing behaviour. Thus, for the symmetric equilibrium, we could drop the subscripts in the equation: $p_{i j}(U)=p_{k j}(U)=p_{j}(U)$.

\subsubsection{Correspondence between QRE and BNE}

Although the derivation of the two equilibrium concepts might look different, they share similar assumptions and choice rules. In both models the following assumptions are used: (1) $\varepsilon_{i}$ are independent for all $i \in N$, (2) the basic payoff functions $U_{i}\left(s_{i j}, s_{-i}\right)$ and densities distribution $f(\varepsilon)$ are common knowledge, and (3) the vector $\varepsilon_{i}$ is $i$ 's private information that is unobserved and not known for sure by either modeller or other individuals. Furthermore, they use the same choice rule that, in equilibrium, each individual chooses the strategy giving him the highest expected payoff, conditional on his own payoff perturbations and his (correct) beliefs about the distribution of his opponents' behaviour. Moreover, they both are equilibrium models in the sense that an individual's choice strategy does take into account other individuals' error (or their types) and therefore is an optimal response to the probability distribution of other individuals' actions. They share that, in equilibrium, behaviour is characterized by a probability distribution that satisfies the "rational expectations" consistency condition: the beliefs that determine an individual's expected payoffs match the decision distributions that arise from applying a probabilistic choice function to those expected payoffs.

A Bayesian equilibrium is based on symmetric uncertain beliefs that individuals have about the other individuals, since the uncertainty arises from incomplete information about their exact type related payoffs. This minor private information, which changes payoffs only slightly, indicates that other individuals choose an optimal action without randomization but with the highest payoffs. In Bayesian games, once the individuals' type-contingent strategies have been computed, each individual behaves as if he were facing mixed strategies by his opponents. As a modeller, we only observe aggregate behaviour, which appears to be a mixed strategy. 
A quantal response equilibrium focuses more on the choice probability of the other individuals; the uncertainty arises from imperfect rational decision. In the QRE model, individuals are rational strategic actors, who might make mistakes in playing and who are aware that other individuals also behave strategically, but are not perfect utility maximizers. QRE assumes that behaviour is inherently stochastic and predicts the probability distribution of aggregate behaviour. Aggregate behaviour is consistent with the interpretation of mixed strategy equilibriums. An alternative way to look at this is that QRE is an equilibrium of beliefs.

Hence, the key difference is that BNE is based upon a model in which individuals know their own payoff functions precisely, but have stochastic beliefs about other individuals' strategic choices, while QRE is based upon a model in which individuals have stochastic knowledge of their own payoff functions. QRE does not specify what the sources of error in the payoff functions are. But given that the components that determine payoffs are assumed three various sources of error may exist (see the section about quantal response equilibrium). Thus, QRE also can capture uncertainty about other individuals' strategies as included in BNE. A prediction that any fixed non-Nash profile will occur implies that at least one individual will make a mistake or has additional private information, either in his prediction of his opponents' play or (given that prediction) in his optimization of his payoff. For a modeller, these cannot be distinguished.

As McKelvey and Palfrey (1995) showed, for a particular distribution of perturbations, under certain assumptions, a QRE is a Bayesian Nash equilibrium of the resulting perturbed game of incomplete information. Nyarko (2001) gave an example that shows how the payoff shocks in QRE can be viewed as types in a Bayesian model. In general, QRE and BNE are equivalent but competing ways of viewing the same situation. Figure 3-3 gives an illustration of the relation between type (error) and strategy, involving two alternatives. Both alternatives bring an inherent payoff of 5. During the comparison, one alternative (A) has the fixed payoff of 5, and the other alternative (B) has an expected

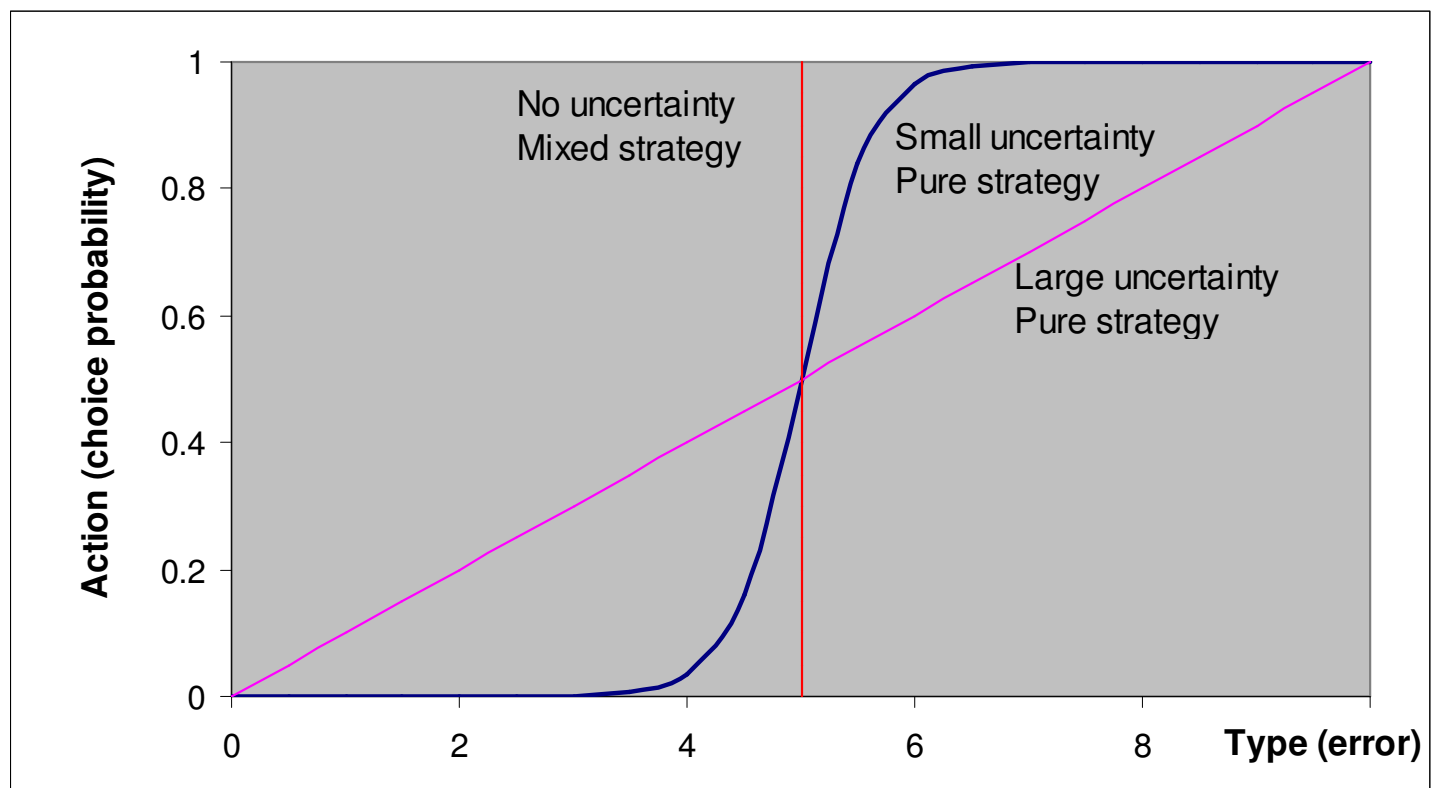

Figure 3-3 Relation between type and strategy 
payoff ranging from 0 to 10 to reflect different payoff assessments of different types. The figure plots the choice probability for alternative B. When there is no uncertainty, both alternatives have a payoff of 5 , and each will be chosen with a probability of 0.5 (that is, coincide with a mixed strategy). When alternative B is assessed with a small uncertainty that indicated a small deviation from a payoff of 5 , alternative $B$ will be chosen more often than alternative $A$ if the expected payoff is higher than 5 , and vice versa. When alternative B is evaluated with a large uncertainty, the choice probability of alternative B will change as dramatic as the difference between the two alternatives.

When we take the Bayesian Nash equilibrium as the way to transfer incomplete information to imperfect information that causes the imperfect decision, it is equivalent in the resulting mathematic equation to QRE. There is a correspondence between QRE and $\mathrm{BNE}$ in the sense that a quantal response is a probability distribution of action profiles in a Bayesian Nash equilibrium. Because QRE is more widely used in empirical applications (Anderson, et al., 1997; Capra, et al., 1999), we will refer to our model as QRE from now on.

\subsubsection{The logit approach}

The particular characteristics of the model depend on the distribution of the random component. A uniform distribution results in a linear model, whereas a normal distribution yields a probit model. Assuming individuals' types are independently and identically distributed (IID), following a Gumbel (or double exponential, or type I extreme value ) distribution, gives rise to the logit model, in which case the choice probabilities of individual $i$ choosing alternative $j$ are a proportional exponential function of expected payoff. That is:

$$
p_{j}^{*}=\frac{\exp \left(E\left[\bar{U}_{j}\right]\right)}{\sum_{j^{\prime}=1}^{J} \exp \left(E\left[\bar{U}_{j^{\prime}}\right]\right)}
$$

This model can represent different scenarios depending on the structure of the interaction between individuals and the degree of uncertainty. Because discrete choice models have found ample application in choice modelling, we will further explore this model.

This model (or equivalent variations of it) can be derived in a great number of ways. Its original formulation is due to Luce (1959), who made assumptions about the choice probabilities rather than about the disturbances. Before discussing the derived equation directly, we will first review some properties of the Gumbel distribution that will be used in subsequent sections. These properties and relevant proofs or more detailed description can be found in, for example, McFadden (1976) and Ben-Akiva and Lerman (1987).

If the prior distribution follows a type I extreme value distribution, the equation for the cumulative density distribution function used here is: 
$F\left(\varepsilon_{i j}\right)=\exp \left(-\exp \left(-\frac{\varepsilon_{i j}}{\mu}\right)\right)=e^{-\frac{\varepsilon_{i j}}{\mu}}$

and the probability density function is:

$f(\varepsilon)=\frac{1}{\mu} e^{-\frac{\varepsilon}{\mu}} \exp \left(-e^{-\frac{\varepsilon}{\mu}}\right)$

where $\mu$ is a positive scale parameter and this distribution has the following properties:

1. The mode is 0 .

2. The mean is $\gamma / \mu$, where $\gamma$ is Euler constant $(\sim 0.577)$.

3. The variance is $\pi^{2} / 6 \mu^{2}$.

4. The Gumbel distribution is an approximation of the normal distribution with "fatter" tails.

5. In the case of linear-in-parameter utilities, the parameter $\mu$ cannot be distinguished from the overall scale of the $\beta$ 's. When $\mu=1$, it is corresponds to assuming that the variance of $\varepsilon$ is equal to $\pi^{2} / 6$, implying that $\operatorname{var}\left(\varepsilon_{i}-\varepsilon_{j}\right)=\pi^{2} / 3$, which is different from the standard scaling $\operatorname{var}\left(\varepsilon_{i}-\varepsilon_{j}\right)=1$.

Let $\mu$ be a positive "error" parameter that reflects the uncertainty of individuals' beliefs about other individuals, which is inversely related to the size of the error variance in the model. The model can then be expressed as:

$$
p_{j}^{*}=\frac{\exp \left(E\left[\bar{U}_{j}\right] / \mu\right)}{\sum_{j^{\prime}=1}^{J} \exp \left(E\left[\bar{U}_{j^{\prime}}\right] / \mu\right)}
$$

This indicates that higher values of the error parameter make choice probabilities less sensitive to expected payoffs. Note that, in strategic choice models like this, $\mu$ indicates the level of concentration of the priori distribution around 0.

\subsubsection{The error parameter}

Figure 3-4 provides an illustration, involving two alternatives with expected payoffs of 5 and 2 respectively. In most empirical studies of individual choice behaviour, $\mu$ is not identified/estimated separately from the remaining parameters of the payoff function. If it is normalized to one, resulting the traditional MNL model to represent individuals' beliefs about activity participation. In fact, assuming each individual has the same value of $\mu$ 


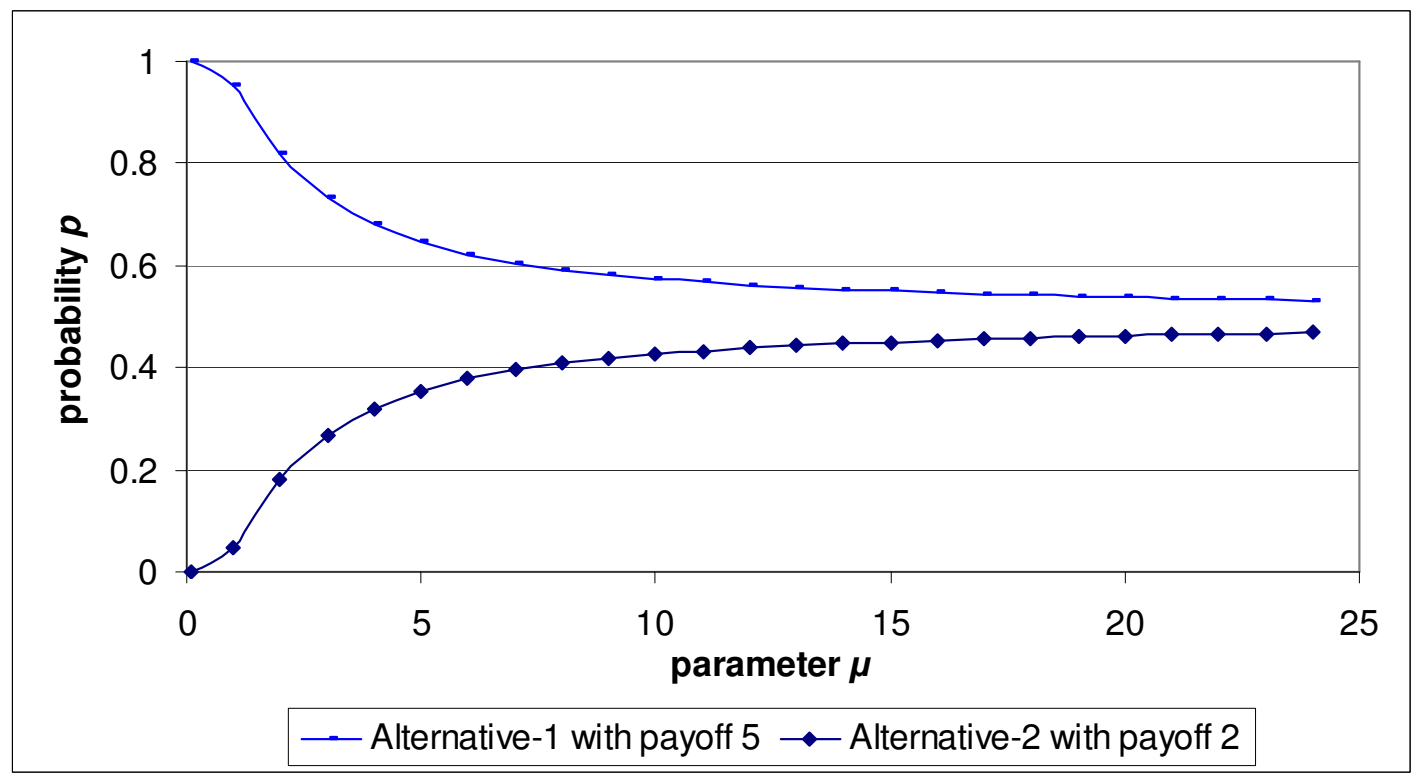

Figure 3-4 Error parameter effect

implies that the variances of the random components of the utilities are equal, proportional to the variance in the error term of the multinomial logit in which $\mu=1$, which can be derived by taking ratios of probabilities in equation (3.8): $p_{j}^{*} / p_{j^{\prime}}^{*}=\exp \left(\left(E\left[\bar{U}_{j}\right]-E\left[\bar{U}_{j^{\prime}}\right]\right) / \mu\right)$.

In the extreme case, as $\mu$ tends to zero, the variance of the utility disturbances approaches zero. Utility draws across time units are concentrated at the distribution mean. In this case, there is no uncertainty about other individuals' utility, and a deterministic choice model is obtained because all the information about individual preferences is included in the systematic utilities. This means there is no error, which approaches the Nash equilibrium of a complete information model. The strategy with the highest expected payoff is selected with probability one, which is equivalent to perfect rational behaviour. The Nash equilibrium is a state of rest in the sense that no individual would want to change its own strategy unilaterally, knowing what strategies others are using.

As $\mu$ trends to infinity, the variance of the disturbances approaches infinity. The probabilities go to equality for all alternatives, irrespective of the payoffs. The choice model then provides no information, so the alternatives are equally likely. That means that actions are based on error only, i.e., there is a unique equilibrium with equal probabilities for each strategy, which is equivalent to perfectly random behaviour.

Extreme cases are useful for providing intuition, in reality, individuals are neither perfectly rational $(\mu=0)$ nor perfectly noisy $(\mu=\infty)$, and it is the intermediate values of $\mu$ that are most relevant for explaining human behaviour. As such, the "error" parameter can be interpreted as a measure that allows us to model the bounded rationality of individuals with limited ability of anticipation.

In some contexts, a small amount of randomness can have a large impact on equilibrium behaviour. Regardless of whether randomness or noise is due to preference shocks, experimental or actual mistakes in judgment, the effect can be particularly 
important when individuals' payoffs are sensitive to others' decisions. In such games, a small amount of noise in decision-making may have a large "snowball" effect when endogenous interactions are considered.

In more complicated and realistic cases, $\mu$ may be different when thinking about the decisions of others and thinking about how others think about a third individual's decision. For the sake of tractability, in our QRE model, we assume that individuals have a precision $\mu$ with which they noisily best respond to their beliefs. The level of $\mu$ is common knowledge; hence individuals rationally expect others to sometimes deviate from perfect decisions.

In general, the amount of noise in the data should depend on the subject pool, the complexity of the strategic situation and the importance of un-modelled factors in the decision-making process. In comparing $\mu$ estimates across experiments, it is important to adjust for the way that payoffs are measured. The error parameter determines how sensitive individuals are with respect to differences in expected payoffs.

The model we described above is used to predict and explain behaviour in strategic situations where individuals' optimal decisions depend on what other individuals are expected to do. Humans are capable of many layers of speculation about possible actions and reactions. When they make choices for the first time, they start with a lot of uncertainty about what others think, about what others think they think, and so on in their iterated reasoning. As they experience playing in a particular situation and make repeated observations about the actual payoffs received from different choices, they can be expected to make more precise estimates of the expected payoffs for different strategies. The notion of equilibrium should be thought of as the limiting outcome of a learning process that clarifies over time through experience.

\subsubsection{Estimation issues}

As stated in the traditional discrete choice model estimation literature, the error parameter cannot be identified because of confounding with the vector of utility parameter. It is the combined effect of $\mu$ and $\beta$ that researchers are estimating, and the estimates of the $\beta$ parameters will compensate for holding $\mu$ constant at a different value. This is also true for the strategic choice model we described above. But in strategic choice modelling, there are two streams of literature with a different interest: one focuses on estimating the impact of substantive covariates on choices; the other is primarily concerned with using observed strategic choices to estimate the degree of error present in individuals' play. There are two corresponding approaches to fitting QRE models to naturally occurring or laboratory data. The primary difference lies in which parameters in the model are restricted and which are estimated. In both cases, maximum likelihood estimation (MLE) techniques are used to fit parameter values to a QRE equation.

\section{Fitting $\beta$ and restricting $\mu$}

In naturally occurring data, the utility values of payoffs in a strategic situation are generally unknown, although we may have theoretical reasons to believe that certain factors covariate with utility. The $\beta$ parameters are fitted using MLE, implying the usual 
interpretation of a multinomial logit model: it is the marginal impact of the matrix of covariates $\boldsymbol{X}$ on the odds ratio between any two alternatives in the model. In this approach, $\mu$ (the degree of error) is typically restricted to 1 as is typically done in the non-strategic choice literature.

In fact, utility is an arbitrarily defined scale, and all that matters is the difference between the two alternatives, reflecting the relative nature of the utilities. Adding a constant to a utility of each alternative does not affect the choice probabilities in the logit model, as multiplying by a positive constant does not in a power model. Hence, to make systematic utilities unique, we must "fix their scale". This is typically done by placing some restrictions on the disturbance. In traditional logit choice models, $\mu$ is commonly set equal to 1 because this is analytically and computationally convenient.

In the case that using linear function with marginal utility of $\theta$ for the interaction component, by measuring the magnitude of $\theta$ and $\beta$ 's, we can examine the relative importance of the endogenous impact versus exogenous impact, and make correct conclusion about whether or not it is essential to using an strategic choice model for the investigated situation.

Signorino (2003a, 2003b) shows examples of international conflicts to demonstrate that by omitting important variables from the interaction term in strategic situations and by fitting non-strategic models to strategic data, the resulting estimations can be significantly misleading, and cause one to draw wrong conclusions. It is also evident form Carmer's work (2003) that if the specification of the systematic component is not appropriate, it will increase the variance of the random component, acting as rescaling and causing a downward bias in the estimator of the $\beta$ 's. By thinking more carefully about what "goes into" the $\varepsilon$ 's we might be able to improve our model.

\section{(2) Fitting $\mu$ and restricting payoff values}

When the payoffs to individuals in the model are known, as in typical laboratory game experiments, the utility of the outcome could be taken as equivalent to the observed values. Hence, MLE chooses the degree of error that makes QRE's prediction match the data most closely. The goal of this sort of estimation is in determining whether (and how accurately) QRE can predict behaviour in a given situation, and deriving the most appropriate value of $\mu$ to use in future predictions of similar situations.

The connection to statistical equilibrium theory is based on the notion that better strategies are more likely to be played than worse strategies, but best strategies may not always be played, due to various sources of error in individual's evaluations of the different strategies in the game. The link between the Nash equilibrium and the quantal response equilibrium is that the strict best-response (optimization) assumption is replaced with a stochastic better-response, as expressed in the relation between their systematic beliefs and the consistency of their choices (McKelvey and Palfrey, 1995, 1998). Since the Nash equilibrium is a limiting case of the QRE, estimates of the error parameter can be used to evaluate the extent to which actual behaviour is close to the Nash prediction (perfect rationality with complete information).

Sometimes, the estimation of $\mu$ over different time periods in the same data set can reveal a learning process. As a game is frequently repeated over time, we can expect 
individuals (by virtue of perfecting their own strategies or getting better information about the strategies of others) to converge to a Nash equilibrium over time. McKelvey and Palfrey (1995) revealed such a pattern in various experimental games (e.g., two person zero sum games): estimates of $\mu$ early in the game tend to be much higher than estimates later in the game, revealing that individuals tend to be less error-prone as the game progresses.

The assumption that the disturbances are independent and identically distributed (IID) represents an important restriction. First, it constraints all the disturbances to have the same scale parameter $(\mu)$. Although the choice of $\mu$ is arbitrary since it simply sets the scale of the utilities, the fact that each disturbance has the same value of $\mu$ implies that the variances of the random components of the utilities are equal. It reflects the dispersion of the random component. Further, the assumption of independence of the disturbance may, in some situations, be difficult to defend. For example, when there is some similarity of strategies or there is some other basis for one strategy's expected utility to affect other strategies differently. Furthermore, it is also possible that people show conformity pressure in their behaviour if their social-spatial network is not explicitly included in their characters (e.g., a certain group of individuals tends to make certain choice more often.)

\subsubsection{Inconsistency: imperfect expectation in one-shot situation}

The suggested equilibrium model considers the case of bounded rationality that individuals have to solve the vagueness when they make decisions. The model described above focuses on the fixed-point intersection, which maps belief probabilities into strategic actions that occur with the same probabilities. This is more appropriate when individuals learned from repeated interactions what others could be expected to do. One might start questioning how individuals behave in a new strategic situation, or what they do when they only meet once like in one-shot game. The answer is all they can do is think. The rational expectations described above might not be appropriate in these cases. The iterated thinking could be considered as introspection. In the introspection process, the belief distributions unlikely coincide with decision distributions. And it is more likely that people are more and more uncertain about what others think, about what others think they think, etc. Following Goeree and Holt's method (2004), some noise can be injected into the model via the logit choice function:

$$
p_{i j}=\Phi_{i j}^{\mu}\left(B^{1}\right)=\frac{e^{\bar{U}_{i j}\left(B^{1}\right) / \mu}}{\sum_{j^{\prime}=1}^{J_{1}} e^{\bar{U}_{i j^{\prime}}\left(B^{1}\right) / \mu}}
$$

where $p_{i j}$ represents an individual's choice probability $\left(B^{0}\right), B^{1}$ represents an individual's (first-order) belief about the other's choice, $B^{2}$ represents an individual's (second-order) belief about the other's belief about the individual's own choice, etc. 
An individual's first-order belief is modelled as the other's logit best response, $B^{1}=\Phi_{i j}^{\mu_{1}}\left(B^{2}\right)$, given the individual's second-order belief, $B^{2}$. In other words, the process that produces an individual's first-order belief about what the other will do is modelled as the other's noisy best response given what the individual thinks the other thinks the individual will do. Thus, the higher-order beliefs follow logit best responses for $(n>k)$ is:

$\Phi_{n}^{k}=\Phi^{\mu_{k}} \circ \Phi^{\mu_{k+1}} \circ \ldots \circ \Phi^{\mu_{n}}$

which maps an individual's $n$-th order belief into his $k$-th order belief: $B^{k}=\Phi_{n}^{k}\left(B^{n}\right)$. Noise in this successive iteration increases to infinity as the number of iteration increases $\mu_{\infty}=\infty$. The set of noisy rational responses is obtained by recursively applying the $\Phi^{\mu}$ mapping to $P$ as the limit, using a higher error rate at every step:

$$
B^{0}=\lim _{n \rightarrow \infty} \Phi_{n}^{0}\left(p_{0}\right)=\lim _{n \rightarrow \infty} \Phi^{\mu_{0}} \circ \Phi^{\mu_{1}} \circ \ldots \circ \Phi^{\mu_{k}} \circ \Phi^{\mu_{k+1}} \circ \ldots \circ \Phi^{\mu_{n}}\left(p_{0}\right)
$$

The probability $p_{0}$ can be chosen arbitrarily because the logit best response $\Phi^{\mu}$ for $(\mu=\infty)$ maps any initial belief probability to a uniform probability (perfect randomness). That is, individuals' higher-order beliefs about what others think about what others think they think about ... etc, become more and more diffuse and these (infinite) thought processes start out with uniform beliefs. The outcomes of these thought processes, individuals' decision choice probabilities, are not uniform. This is different from equilibrium concepts that map belief probabilities into actions that occur with the same probabilities.

Goeree and Holt (2004) assumed the error rates to grow with each iteration according to a two-parameter function, which has some special properties that link to our rational model:

$$
\mu_{k}=t^{k} \mu_{0}
$$

where $k$ is the iteration reasoning order. The parameter $t$ determines how fast the noise increases with further thinking iterations. When $t=1$, the limit probabilities, $p^{*}$, must be invariant under the logit map: $\Phi^{\mu_{0}}\left(p^{*}\right)=p^{*}$. A fixed point of this type constitutes a logit equilibrium, and leads to a connection with our rational expectation model as expressed in equation (3.8).

In the extreme, when $t$ is very large, the model corresponds to one with all individuals involved in one-step reasoning and thinking that others make a random choice. When $t>1$, the choice probabilities do not match the belief probabilities during iterative thinking, and the introspective process allows for surprises, which are likely to occur in scenarios that involve unique, non-repeated interaction. Given the payoff parameters of the model, the introspective process in (3.9) predicts the probability with 
which an individual chooses strategies in one-shot situations. And this prediction will accommodate a wide range of iterative reasoning about beliefs over beliefs, regarding the values of $\mu_{0}$ and $t$.

\subsubsection{Interpretation and application}

To see how far the model can take us, we need to apply it to a specific context. For example, as demand is increasing, many facilities (such as museums and restaurants) face limitations in their ability to serve consumers during the most popular visiting time slot. The utility of visiting these kinds of facilities may depend on, for example, the queues that one experiences. Consumers need to make a strategic trade-off between their own timing preferences and the anticipated congestion based on the trade-offs that others are likely to make. In the extreme, if all consumers have the same timing preference and a strong dislike of congestion, but believe that others do not dislike congestion, no consumer may end up using the service at the most preferred time. Alternatively, if none of the consumers anticipates congestion, all consumers may find themselves using the service at the same most popular (and most congested) time slot. Our model is capable of explaining these kinds of phenomena - in which the interaction of many individually rational decision-making processes produces an outcome intended by none of them.

\subsubsection{Understanding mixed-motives}

In social environments, people make choices with uncertain outcomes. These outcomes are interdependent in the sense that the outcome depends on how others decide. In the context of spatial choice, these interdependences may derive from: (1) events separated in space and time; (2) people within households or people outside; (3) sequences in activity travel patterns. The essential feature of social interaction is that each decision maker has only partial control over the outcome. That is, what a person does has consequences not only for himself, but also for others, and this is true at the same time for other individuals.

The interpretation of behaviour in the context of social interaction is inherently problematic. Most social interactions are based on mixed-motives - the preferences of individuals neither perfectly coincide nor are entirely opposite - but involve cooperation versus competition, trust versus suspicion, individualism versus collectivism, selfishness versus altruistic and fairness (Fehr and Schmidt, 1999; Fehr and Fischbacher, 2002). Therefore, an important structural property of the model is the way in which individuals' payoff functions are related to one another. By different specification, cooperation choice may reflect attempts to maximize either joint payoffs or individual payoffs on the basis of trust, while defection choice may reflect attempts to maximize either individual payoffs on the basis of mistrust or relative payoffs.

\subsubsection{Application issues: user, domain and data}

The economic value of a theory is measured by how much the model's forecasts improve the profitability of a particular individual's choice. At the individual level, the proposed model can serve as a personal assistant to support individuals making smarter decisions 
by more accurately predicting what others will do. These predictions can be used to compute expected payoffs of strategies, and recommend a choice with the highest expected value. At the planner's level, the proposed model can be used as a proxy of the population to assess the feasibility of planning actions or to predict external effects on existing destinations through the distribution of flows. It can be extended to support more complex decision problems; for example, individuals like a tourist office may wish to influence the behaviour of individual tourists to optimize or at least improve some system-level objective. In that case, any model should also take into account the extent to which individual tourists comply with the provided recommendations and tourists' anticipation of the extent to which other tourists will comply. In that case, the proposed model can be used as a building block for a more complex model.

The model can deal with a broad range of (spatial) choice problems from a one-shot game to repeated choices towards equilibrium, depending on the characteristics of the choice behaviour under investigation. We give a brief list of potential applications related to commuting, spatial shopping behaviour, residential choice behaviour and recreational behaviour.

In one-shot games, individuals use noisy introspection - an iterated quantal response function with a response error parameter increase in an iterative reasoning process to arrive at some choice. This type of behaviour seems especially appropriate to model recreational behaviour such as first time visits to a theme park or a new destination. Housing choice and location choice of firms that happen less frequently may also be a proper application area for this model. Another possible application of the oneshot model is to provide initial prior belief for repeated interaction in learning such as an individual migrating to another city or country.

In repeated situations, individuals have had significant experience with a population (or have heard about the general character of the population from organizations or friends). Many problems such as choice of travel mode, departure time, route of commuter behaviour, which are repeated daily, should be addressed using an equilibrium model.

The link between the QRE model and the Nash equilibrium model lies in that the Nash equilibrium model assumes that all individuals behave perfect rationality and that they believe that other individuals behave perfect rational in the same way as they did with the same complete information. There is no error in play. In real life, this outcome is rare, if is not impossible.

\subsection{Models of strategic choice behaviour based on interaction and interdependencies between individuals and information providers}

In Section 3.2, we developed a strategic choice model that focuses on how individuals anticipate congestion between them, when choosing their own activity participation time. When an individual receives information/recommendations about the flow distribution, he has to adjust his anticipations. Provision of such information implies that the decision problem becomes even more complicated. Both individuals and the information provider may act strategically. When indeed information is provided, individuals have to make assumptions about if and how other individuals will adjust their (current) behaviour given the provided information. The information provider has to make similar strategic 
decisions in light of its expectations of individuals' reactions to the information he provides.

This is a bi-level optimization problem, where the information provider tries to achieve his objective, while the individuals maximize their individual payoffs. Since the information provider's recommendation influences the choice of individuals, and the individuals' choices in turn have an impact on the possibilities of the information provider to realize his objective, this is analogous with the strategic situation of a game for two players.

In this section, we first give a game formulation of the problem that we described. Then, we present a behavioural model for the interaction between the information provider and individuals that allows the former to anticipate its interaction with the latter (to integrate the latter's predictable decision process into its own decision making), in order to determine optimal strategies of information provision. The individual strategic choice model that developed in last section is extended in response to information. Finally, various objectives that different information providers may have as well as the application of the model will be addressed.

\subsubsection{Two-player two-stage game formulation}

As explained, the second game is a game at the aggregate level between the information provider and the collective individuals. In this game, there is another player - the information provider. The purpose of the information provider is to influence the use of urban facilities to reach some system level objective, e.g., an optimum level of usage. Adding the information provider to the game is not as simple as extending the described $N$-player game (Section 3.2.1) to an $N+1$-player game, because the strategy space and the payoff function for this additional player differ from the rest of the $N$ players.

In order to investigate the second game, similar to the first game, we use activity participation timing choice as an example. We assume that the individuals' choice behaviour with $J$ available access time slots can be characterized by the Bayesian Nash equilibrium as described in Section 3.2.1. This allows us to treat the collective individuals as one player who distributes the flows among the urban facilities. Thus, a two players non-cooperate game is formulated. The two players have imperfect, certain, asymmetric and complete information. The difference in information from game 1 lies in the properties of asymmetric and complete information, which is relevant to the characteristic of this game. Asymmetric means that one player has more information than the other player when he moves. Complete means each player knows (or observes) the others move before his moves.

Unlike the simultaneous moves of individuals in game 1, this is a two-stage game. First, the information provider sets the strategy. Then, the individuals observe the result of the strategy settings and choose their best choices accordingly. It is assumed that the information provider can choose the best control strategy to achieve the highest system payoff, while taking the anticipated individuals' reactions into account. That is, the information provider can solve the individuals' optimization problem by anticipating the individuals' best reaction to each possible control strategy in selecting the best strategy. In the case of duopoly (two players), such a multi-stage game is known in economics as a Stackelberg game. The player who moves first is the leader, the player who moves next is 
- Players

- The collective individuals and the information provider

- Information

- Imperfect, certain, asymmetric, complete

- Strategy and Events

- Personal choices

- Non-cooperative, sequence move

- Payoffs

- The sum utilities of individuals' choices

- The sum utilities of the flow

- Stackelberg Equilibrium

\section{Figure 3-5 Game 2 formulation}

the follower, and the result is in favour of the leader because of the asymmetric information. The information provider's payoff is a utility function of the overall distribution flow, and the collective individuals' payoff is a utility function of individuals' choices (see Figure 3-5 for a summary).

The upside-down tree structure (Figure 3-6) represents the extensive form of this game. The key characteristics of this multi-stage game of complete information are that the moves occur in sequence; all previous moves are observed before the next move is chosen, and the players' payoffs from each feasible combination of moves are common knowledge.

In general, the equilibrium of this game is not a Nash equilibrium. Rather, the equilibrium is determined by backward induction. The information provider first initiates the move by setting control strategy $g$. When the individuals make their move based on their strategy $h$ at the game's second stage they will face the following problem:

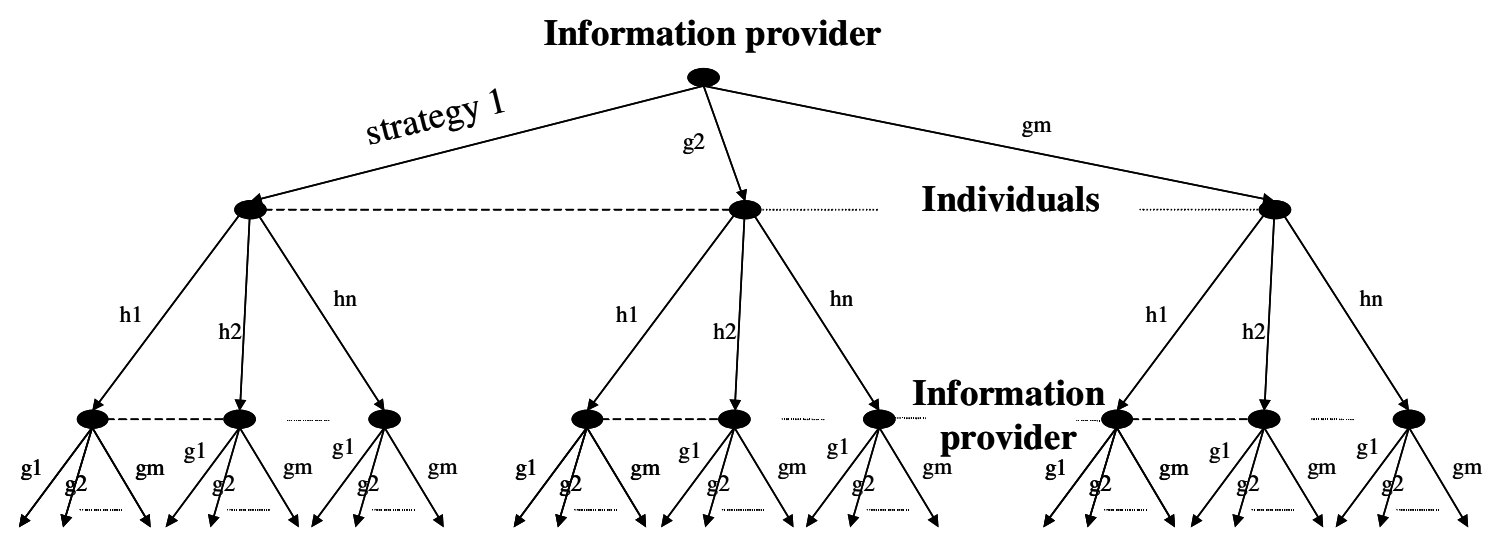

Figure 3-6 Game 2 extensive form 
$\max _{h \subset H} U_{h}(g, h)$

Assume that for each $g$ in $G$, the above optimization problem has a unique solution. Denote the unique optimal solution by $h^{*}(g)$, where $h^{*}(g)$ is the individuals reaction or best response to the information provider's strategy $g$. Since the information provider can solve the individuals' optimization problem, he should anticipate the individuals' best reaction to each possible control strategy $g$. Therefore, the information provider's problem amounts to:

$\max _{g \subset G} U_{g}(g, h *(g))$

The equilibrium of the Stackelberg game can thus be defined as (e.g., Scott Bierman and Fernandez, 1998):

In the two-player game between the information provider and the collective individuals, $\Lambda=\left\{G, H ; U_{g}, U_{h}\right\}$, the combination of strategies $\left(g^{*}, h^{*}\right)$ is a

Stackelberg equilibrium if strategy combination $\left(g^{*}, h^{*}\right)$ solves the following bi-level problem: $\max _{g \subset G} U_{g}(g, h *(g))$, subject to: $h^{*}(g)=\max _{h \subset H} U_{h}(g, h)$.

\subsubsection{Interactive decisions between individuals and information providers}

There is a substantial transportation research literature on the use of information by the information provider to influence the behaviour of individuals in urban planning. Most of these studies concern the idea that providing information may reduce the uncertainty and expose possible hidden alternatives to individuals, to induce individuals to behave in a certain way such that some system-level objectives are satisfied.

In the formulated two-stage game situation, we assume that the information provider takes the lead. His strategy space is the recommendation based on his given objective set (i.e., achieving an equal usage level, overall welfare maximum, or user equity, see section 3.3.4 for a more detailed description). The individuals' strategy is more complicated because it must specify an action for each information set, and this information set depends on what the information provider recommended. A typical example of elements of the individuals' strategy set is (comply, ignore), which specifies that he chooses to ignore if the information provider's recommendation is based on the objective "equal usage level" that is contradictory to his personal objective, and complies if the information provider's objective is "user equity" that coincides with his personal objective.

In the case of a Nash equilibrium of two players, when the information provider has no a-priori knowledge of the reaction of the collective individuals after they receive the information, the iterative process evolves as follows: the information provider offers 
certain information by assuming some usage level, the individuals react and after a shift of start time choices a new user distribution emerges, the information provider observes the shifted usage levels and adjusts his control measures by offering different recommendations, etc. The equilibrium that can be achieved is a Nash equilibrium: no player can improve his objective function by changing his own decision without cooperation of the other player. In this case, it is equivalent to the so-called "Cournot game" that has the characteristic of a Nash equilibrium with two players moving simultaneously. Reality might be a little more complicated because the information that is offered by the information provider is often based on previous conditions. After the information or recommendation is offered, the usage flow may change for the entire time slot, which makes an adjustment of the information necessary.

In a two-stage game, first, the controlling individual sets the strategy and gives a recommendation. Next, the individuals observe the recommendation and choose their optimal start time after updating their anticipations accordingly. In the extreme case that the controlling individual has full control over the game, a so-called "Monopoly game", the system optimum can be reached. In this case, the controlling individual not only sets his strategy, but also lets individuals follow the recommendation completely. The solution of this "Monopoly game" can serve as an ideal distribution for the controlling individual, which gives the best performance from the viewpoint of the controlling individual. In reality, a monopoly solution may not be sustained over the long run since it is not in the individuals' best interest and therefore lacks a behavioural basis.

It is more realistic to assume that the information provider optimizes the offered information by, in advance, already taking into account the likely reactions of the individuals, implying that the problem is reduced to a single level optimization, known as a Stackelberg game (e.g., Fudenberg and Tirole, 1991). However, it is possible that, if information is offered without forward looking, the state emerging after the adaptation process is worse than the initial state before the optimization. In the situation of a information provider representing the supply side with the objective to achieve an equal usage level, and the individual users on the demand side with their own objectives to optimize their activity start time, the above Stackelberg game can be defined and can be solved using the following fixed-point algorithm:

$$
g^{r}=U_{r}\left(g^{r}, H^{*}\left(g^{r}\right)\right) \text { subject to: } H^{*}\left(g^{r}\right) \text { solves } H^{\prime}=U_{H}\left(g^{r}, p^{*}\right)
$$

where $H^{*}\left(g^{r}\right)$ is the individuals reaction or best response to the information provider's strategy $g^{r}$. Mathematically, the Stackelberg optimum can be found by searching for the optimum value of one player's objective function along the optimum curve for the other player (e.g., Basar and Olsder, 1995). In theory, such an optimum is equal to or better than the Nash optimum, which reflects the points where both curves intersect.

\subsubsection{Update of individual's expectation given information provider's recommendation}

Given the problem among individuals described in Section 3.2, each individual will have certain beliefs about the probability of others choosing each time slot and his choice 
probability is derived from and coincides with these beliefs. An individual chooses start time $j$ if the utility he gets from this choice exceeds the utility from all other possible choices (start time $j^{\prime} \neq j$ ). This implies that the prior probabilities indicate the individual's self-expectation of the probability of choosing a particular time slot $j$ for visiting a certain facility, as expressed in equation (3.8).

As we have indicated, individuals will have to update their expectations in light of the recommendation. Not knowing the strategy underlying the recommendation, individuals have to decide on their belief to what extent other individuals will follow the recommendation. We assume that the information is reliable, although it may be "good" or "bad" in the sense that it may or not match an individual's expectations. We assume that after receiving the recommendation, an individual will compare the probability of choosing various time slots calculated on the basis of his own beliefs and the recommendation. Two situations may arise: (i) the recommendation is consistent with the existing expectations, or (ii) the recommendation is incongruent with the existing expectations. If the recommended probability of choosing various time slots is not at all in line with the individual's expectation, it is less likely that the individual will comply, but rather it is more likely that he will stay with his own expectation.

The provided information may take on the form: "There is $60 \%$ chance alternative 1 is a good choice, $20 \%$ for alternative 2, etc.", which is denoted as $g_{j}$ for $j=1, \ldots, J$. The total recommendation for all alternatives should summarize to $100 \%: \sum g_{j}=1$. The collective individuals' response is the weighted sum of self-expectation and the recommendation. If individuals compare their self-expectation with the provided information on the basis of each alternative, the updated anticipation for each alternative, after having received the recommendation is expressed as follows:

$h_{j}=\left(1-q_{j}\right) p_{j}^{*}+q_{j} g_{j}$

where $h_{j}$ is the updated probability for alternative $j$ and used as input for the information provider to assess the objective achievement, and to revise the recommendation to optimize the expected outcome. The first term on the right side indicates the self expected probability for alternative $j$ and the second term represents the recommendation probability for the same alternative. Parameter $q$ is the index of caution (versus boldness) and characterizes the degree to which the individuals are willing to follow the recommendation (risk aversion versus risk taking behaviour).

In general, $q$ could be defined in a number of ways. As noted in Arthur (1994), humans carry out localized deductions based on their current beliefs and act on them. And, as information from the environment comes in, they may strengthen or weaken their beliefs underlying their current conjectures, discarding some when they cease to perform, and replacing them as needed with new ones. They may be more conservative to the information that is far away from their current beliefs, and more easily integrate the information that is close to their anticipation (e.g., Srinivasan and Mahmassani, 2000). Given these characteristics, the compliance function can be defined as a function of discrepancy between own beliefs and recommendation, which will decrease as the difference between self-expectation and recommendation increases. That is, 


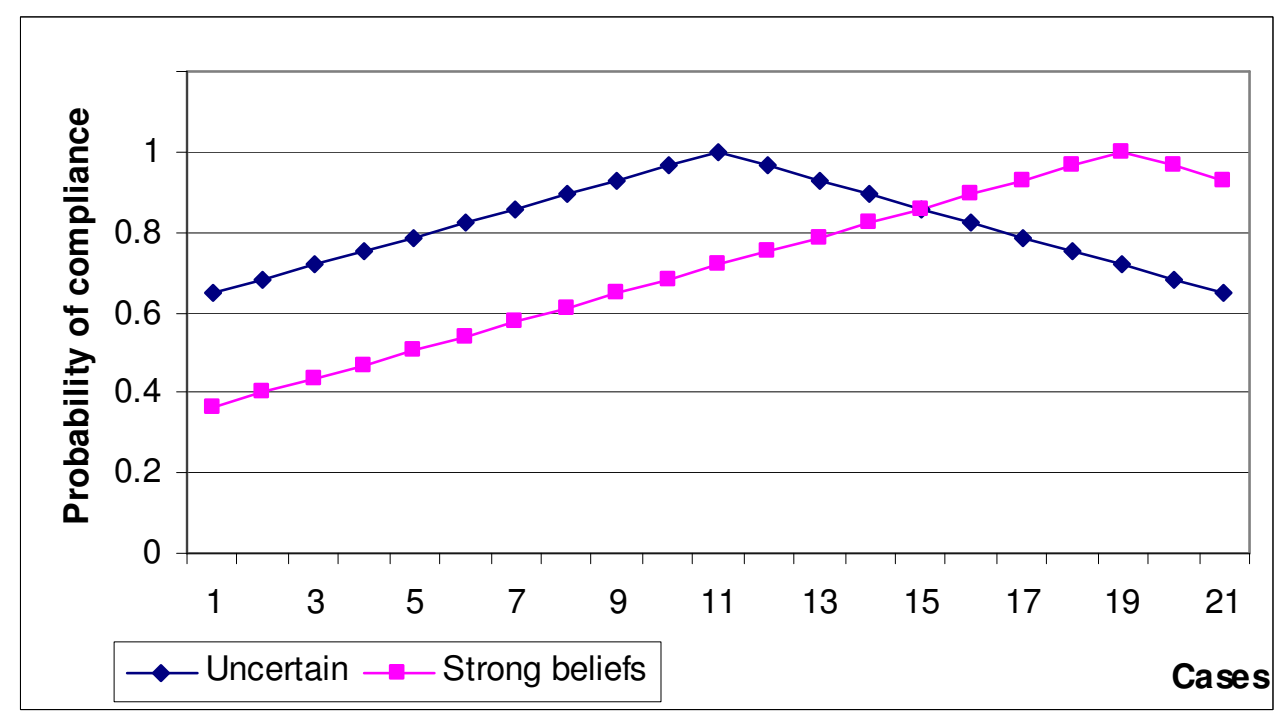

Figure 3-7 Effect of self-expectation on compliance rate

$q_{j}=1-\frac{1}{2} \sqrt{\left(g_{j}-p_{j}^{*}\right)^{2}}$

In the extreme case, as the recommendation $\left(g_{j}\right)$ matches the self-expectation $\left(p_{j}^{*}\right)$, the recommendation is a confirmation of the self-expectation and the compliance will be $100 \%$. In the other extreme, as the recommendation is fully incongruent with the selfexpectation (e.g., $g_{j}=0 \%$ versus $p_{j}^{*}=100 \%$ ), the compliance is $0 \%$. An illustration is given in Figure 3-7, where the recommendation increases gradually form $0 \%$ to $100 \%$, while in the uncertain series the self-expected probability is fixed at $50 \%$, and in the strong belief series set at $90 \%$.

\subsubsection{Information provider's objective}

Knowing the response of the individuals, an information provider could choose the best strategy to provide the recommendation in order to influence the usage distribution. Based on the different kinds of objectives, the recommendation provided to the collective individuals will differ. Three basic kinds of typical information provider can be distinguished. The primary difference lies in the different assumptions about the information provider's payoff functions that determine the outcome of the equilibrium.

\section{(1) Equal usage: equal distribution across all time slots}

If the information provider represents a facility manager (e.g., restaurant owner, museum, etc.), the ideal situation may be that the visitors are uniformly distributed across the opening hours, based on efficiency criteria. The facility utility could be related to the 
visits according to a continuous function (for instance, a monotonic convex nondecreasing function). However, the facility has its capacity constraints. When the number of visits exceeds the saturation level, the facility utility may drop because of extra labour or maintenance cost. Moreover, the flow of visits is not stable, but rather fluctuates, implying that a facility, which is too crowded or too empty, is not efficient. Therefore, this utility function depends on both the flow of visits and stability, satisfying the requirements of being continuous and differentiable with respect to flow and degree of saturation.

If the information provider's objective is to achieve a equal usage level, the objective function could be measured in terms of the deviation from the reference points:

Max: $U_{r}=\sum_{j=1}^{J} X_{j}$
subject to: $\quad X_{j}=\bar{x}-\alpha_{1} \max \left\{N / J-N h_{j}, 0\right\}-\alpha_{2} \max \left\{N h_{j}-N / J, 0\right\}$

where $X_{j}$ is the facility utility of time unit $j, \bar{x}$ is the utility of the ideal situation, which in this case is reached when the number of visits is equal to the average, $\mathrm{N} / \mathrm{J}$, and which can be simply normalized to 0 as a reference. $N h_{j}$ is the number of individuals that will shown up at time $j$, given the recommendation.

For simplicity, we assume that the facility utility function is linear in the number of individuals. This implies that the marginal rate of an additional individual is constant. This may not be realistic, but it may show its robustness in that most experimental observations can be explained on the basis of this very simple utility function. Further, we can allow $\alpha_{1} \geq \alpha_{2}$, which reflects the idea that a facility suffers more loss from less visitors: that is, negative deviations from the reference count more than positive deviations.

\section{(2) Welfare maximum: maximum utility across all individuals}

An alternative objective of the information provider may be welfare maximization. This implies that the information provider represents a government agency, which acts neutral and attempts to maximize the total payoff across all individuals, including the case that one individual's welfare is compensated by another's. This may, for example, apply to a tourist body, representing various individual service outlets. This objective can be expressed as:

$\operatorname{Max}: \quad U_{r}=\sum_{j=1}^{J} E\left[\bar{U}_{j}\right] N h_{j}$

where $E\left[\bar{U}_{j}\right]$ is the average expected payoff for alternative time $j, j=1, \ldots, J, N h_{j}$ is the number of individuals that will shown up at time $j$. 


\section{(3) User equity: same utility for all individuals}

Alternatively, the objective may be to achieve a distribution of utilities that is as equal as possible for all individuals across all times (e.g., equal chance to get access to the facility at most popular times), which indicates that the information provider represents the collective set of individuals. This equity objective will coincide with the user optimum in the sense that no single user can improve his payoff function by unilaterally changing his choice. Thus,

$\operatorname{Min}: \quad U_{r}=\sum_{j=1}^{J}\left(E\left[\bar{U}_{j}\right]-\frac{1}{J} \sum_{j=1}^{J} E\left[\bar{U}_{j}\right]\right)^{2}$

where $E\left[\bar{U}_{j}\right]$ is the average expected payoff for alternative time $j, j=1, \ldots, J$.

It is also possible that the information provider represents some associated parties. In this case, an urban planning authority could have some combined objectives such as, for example, trying to achieve equal usage for the supply side, but also trying to achieve maximum welfare for the demand side. A straightforward way of dealing with this problem could be to give the basic objectives some weight and add them together. It would be interesting to slowly modify the respective weights to see how optimal solutions change. The problem, however, is to determine the weights, which is beyond the scope of this thesis.

\subsection{Conclusion and discussion}

This chapter has described and exemplified the application of models of strategic choice behaviour. By highlighting the interdependent nature of people's behaviour in a social and physical environment, it extends traditional research with a focus on individuals' response to an exogenous environment to the role of the endogenous interaction between different decision makers.

In strategic situations, people care about what others are likely to do. In the last few decades, a large body of mathematical "game theory" has been developed about how people will make choices in these situations. However, these theories generally assume that people know a lot about how other people are likely to behave in these strategic situations. In reality, however, people usually figure out what others are likely to do by learning from experience. Reasoning about others may be limited because individuals are not certain about other individuals' payoffs or degree of rationality nor do they know the environment with certainty.

We formulated the interaction problem between individuals at the disaggregate level as a $N$-player $J$-option game and used a discrete choice model with an endogenous interaction term to describe the strategic choice behaviour of a given individual, in which the choices of other individuals are explanatory variables in the equation. We differentiated between three static stages of evolving behaviour in strategic situations: (1) expectation with complete information in ideal situations - Nash equilibrium, (2) expectation with incomplete information in repeated situations - quantal response equilibrium, and (3) expectation with incomplete information in one-shot situations - 
noisy introspection. The model is a general framework in the sense that it leaves open alternative specifications of the interaction components, and allows different decision styles: from perfectly rational behaviour to completely random behaviour. We use the logit (exponential) probability response function to transform expectations of strategies into choice probabilities.

We further expanded this model to address the problem of strategic interaction between an information provider and the collective set of individuals. By formulating a two-player two-stage game with a Stackelberg equilibrium, the information provider gets the leader's advantage without losing the behavioural basis. We extended the developed strategic choice model to predict individuals' response to information provision and distinguished between different roles of the information provider.

In the next chapter, we will use numerical simulation to examine the model's properties. The face validity of the model, that is, whether the simulations are sensitive to possible violations of the formulated assumptions, will be tested. The rigorousness and sufficiency of the model specification will be assessed by comparing the consequences of alternative assumptions. 


\section{Chapter 4}

\section{Numerical Simulation}

\subsection{Introduction}

In the previous chapter, we described the conceptualization and specification of a strategic choice model, which incorporates the impact of aggregate choice probabilities on individual choice decisions. These choices were formulated as the outcome of a game involving incomplete information, in which individuals have to conjecture the behaviour of other individuals. It was further developed to model dyad behaviour of an information provider and the collective set of individuals. It is concerned with the problem how individuals' choices under guidance information aggregate to form an equilibrium distribution, which in turn influences guidance generation and determines the best possible achievement of the policy objective of the information provider. These choices were formulated as the outcomes of a Stackelberg game.

In this chapter, we will explore and investigate the properties of the proposed model using numerical computer simulations. As noted in Engemann, et al. (1989), a computer simulation is helpful in the development of a theory/model. The rigorousness and sufficiency of the theory/model specification can be assessed by facilitating the derivation of testable hypotheses, and making it possible to compare consequences of alternative assumptions (that is, sensitivity analysis). At this stage, the primary goal of the simulation is to test the developed theory for various facets of validity. That is, we examine the proposed model on the basis of the following research questions: 
- Sensitivity to parameter settings: when employing different parameter values does the model produce distinguishable choice behaviour?

- Face validity: if the above is the case, are the different results interpretable in terms of intuition and theory?

We start with examining the model of interactive decisions between individuals, continuing with the model of interactive decisions between individuals and an information provider, given alternative objectives and different levels of expectations. To illustrate the working of the model, it is applied to individuals' start time choices for visiting a facility in a city. Optimal start times represent a strategic advantage for individuals because they can choose different start times to derive more utility from the activities they choose. Empirically, while many individuals choose an activity starting at originally preferred times, there are also individuals that choose to start at less preferred times in order to avoid crowdedness or congestion. The predictions of the proposed model are built on some primitives - the preferences across heterogeneous individuals and the underlying reaction to provided information. It is expected that the presence of more individuals during the same time slot has a negative effect on payoffs. An assessment of how the different components are incorporated in individual's decision making and how well the information provider's objective is achieved by providing recommendations to influence individuals' decision-making can be useful to effectively estimate the model from empirical data.

\subsection{Interactive decisions between individuals}

As a first step in testing our strategic choice model that is based on interactions and interdependencies between individuals, operational decisions had to be made with respect to more detailed settings of the simulation context. To illustrate these decisions, assume that a certain facility is open for 5 hours (every one hour time slot is set as one start time slot), denoted by start time $1, \ldots, 5$, and assume that the average duration of a visit is one hour. In order to focus on the aspects of individuals' interaction, carry-over effects are left out, which means that individuals only take into account the impact of immediate encounters. For an individual who wishes to start visiting at time 3 , the immediate encounters are only those visitors that start at the same time. The remaining individuals who started at time 1 and time 2 and the encounters at time 4 and time 5 will only have very minor effects and are therefore set equal to 0.

In this illustration, for the choice alternative-specific component of the model (equation 3.1), we consider both the start time preference $\left(\beta^{1} \boldsymbol{X}^{1}\right)$ and $\operatorname{cost}\left(\beta^{2} \boldsymbol{X}^{2}\right)$. The start time preference is the attractiveness of conducting the activity during that time slot, whereas cost is the general cost of performing the activity during that time slot. For the interaction component, a linear function with an identical marginal disutility $(\theta)$ is considered. This implies that the negative effects of additional individuals that choose the same start time are additively constant. The number of other individuals that individual $i$ $(i \in N)$ expects to choose to undertake the activity at time $j$ equals $(N-1) p_{j}^{*}$, where $p_{j}^{*}$ denotes the equilibrium probability that other individuals are expected to choose to undertake the activity at time $j$. If the individual chooses time $j$ as well, the expected number of individuals in that time slot will include that individual with certainty and, therefore, the expected number of individuals at time $j$ is increased by one, that is 
$(N-1) p_{j}^{*}+1$.

Based on equation (3.1), the systematic expected payoff function that is used for the illustration $\left(E\left[\bar{U}_{3}\right]\right)$ is given by:

$E\left[\bar{U}_{3}\right]=\beta^{1} X_{3}^{1}+\beta^{2} X_{3}^{2}+\theta\left((N-1) p_{3}^{*}+1\right)$

Obviously, an individual starting at time 1 or time 2 will have fewer encounters during his visit, and more flexibility in terms of duration. An individual who starts at time 5 will also have fewer encounters, but may not have enough time. On the other hand, for some individuals, time 1 or time 2 may be more attractive than time 3 , time 4 or time 5 because they can do the relevant activity first, still allowing for other activities later on. The equilibrium participation pattern is then determined by the trade-off between activity start time preference and expected crowdedness. This reasoning is the basis for the parameter settings used in the simulation. The specific settings will be discussed later. We will first discuss the general algorithm that was used in the simulations.

\subsubsection{Algorithm}

The participation and start time choice was simulated for 1000 individuals, given an assumed preference vector for each start time and the parameters needed in the model. The following procedure was implemented:

1. Draw 1000 sets of random values from a normal density distribution with zero mean and a variance equal to 1 for the 5-dimensional vector of preference. Every set represents an individual's type - his preference deviations from the mean values for the 5 start times respectively.

2. Add the average preference means to the random draws to define each individual utility.

3. Use these values to calculate the probabilities that each individual will choose a certain time slot, using equation (3.8).

4. Sum the probabilities for each start time across all 1000 simulated individuals, generating a distribution of choices for 5 start times.

\subsubsection{Simulation results}

The resulting distributions of individuals' optimal start time strategies represent the probabilities of starting the activity at a particular time rather than the exact start time choice. A very simple scenario was used to start investigating the different facets. It was then extended to show the importance of conjectures about the behaviour of other individuals in driving the decision-making process. By doing that, an attempt was made to get some insight into the trade-off between the cost of deviating from one's originally preferred participation time and the benefit of avoiding crowdedness to explain the sorting pattern of individuals' activity timing decisions. Finally, the simulation was 
extended to demonstrate how uncertainty about others' behaviour will affect one's decision.

\subsubsection{Sensitivity to choice alternative facets}

In general, it is assumed that the individuals' type is reflected in their original timing preferences. Cost brings along disutility. It is expected that the presence of more individuals during the same time slot has a negative effect on the payoffs for that time slot. The arbitrarily chosen values for the parameters used to calculate payoffs were (where $\mu$ was taken from Goeree and Holt (2004)'s results for the QRE):

$$
\begin{array}{ll}
\beta^{1}=1, & \\
\beta^{2}=-0.6, & \\
\theta=0 & \\
\mu=6.6, & \text { (Preference), } \\
\bar{X}^{1}=\{10,10,10,10,10\} & \text { (Cost). }
\end{array}
$$

An initial vector of $\{201,199,200,200,199\}$ was set as the base distribution at the aggregate level for the 5 time slots. By changing one parameter at a time, insight into how these choice facets influence the equilibrium distribution was obtained. In the figures presented in this section, the Y-axis represents the number of individuals simulated to choose each time slot, whereas the $\mathrm{X}$-axis indicates the 5 time slots.

Figure 4-1 shows the accumulating impact of different factors. First, the mean preference in the population was changed from an equal preference pattern for all time units $\bar{X}^{1}=\{10,10,10,10,10\}$ into the following preference pattern $\bar{X}^{1^{\prime}}=\{10,10,3,3,3\}$, reflecting a strong preference of the population for the first two time slots. The preference line in Figure 4-1 portrays the results. Comparing the preference line with the initial line shows that when all conditions are the same, without time-dependent cost differences and interactions between individuals, the distribution of individuals at the aggregate level is proportional to the mean preference pattern for different time slots.

Second, the time-dependent cost was changed from $\bar{X}^{2}=\{10,10,10,10,10\}$, the same costs for all 5 start times, to $\bar{X}^{2}=\{10,10,5,5,5\}$, showing higher costs for the first two time slots. The result is represented by the Wcost-0.6 line in Figure 4-1. It represents a situation where people are willing to pay more to perform an activity during their originally preferred time. The results suggest that when the population has start time dependent preferences and there is no interaction impact between individuals, the time dependent cost can be adjusted to influence the distribution, at least to a certain level. This is the common mechanism used in for example, highway toll systems or the Dutch railway discount hour card system to reduce congestion. 


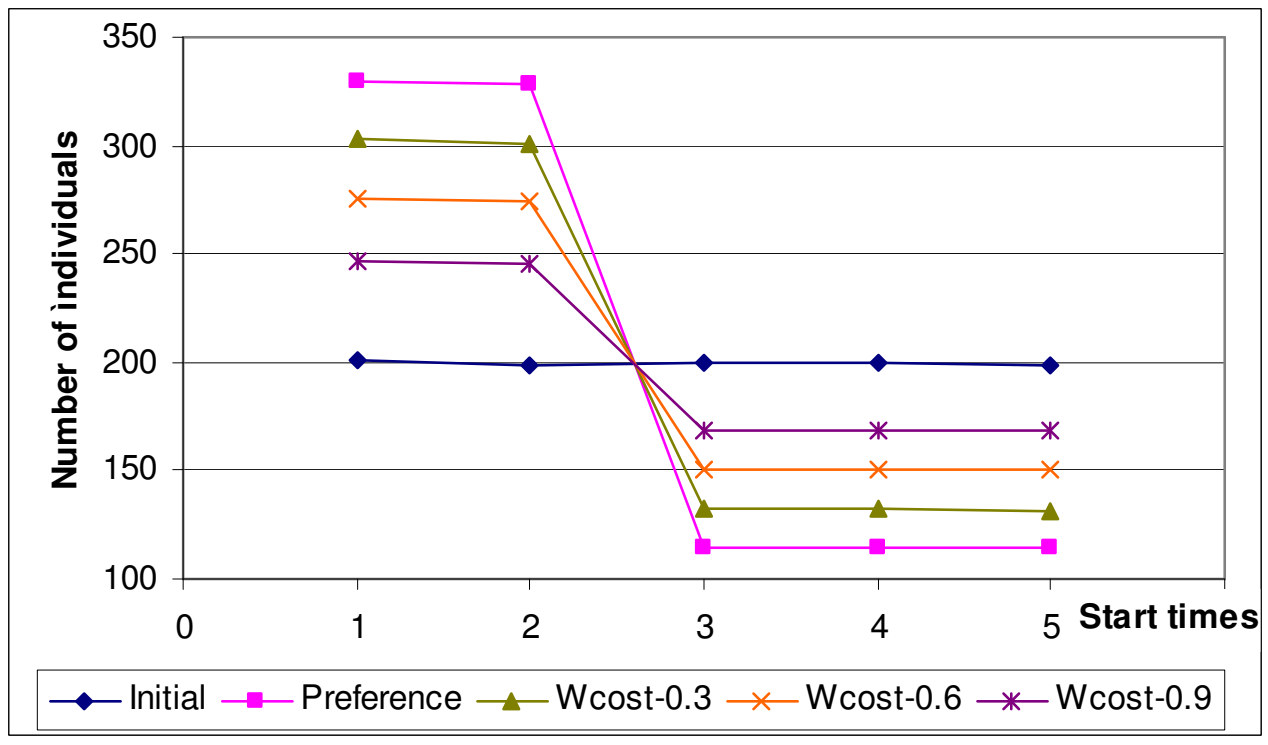

Figure 4-1 Choice alternative impact

Moreover, when the weight of cost is changed from $\beta^{2}=-0.6$, to $\beta^{2}=-0.3$, and $\beta^{2 "}=-0.9$, different sensitivity levels regarding cost among individuals are simulated. The results are displayed in Figure $4-1$ for respectively Wcost- 0.6 , Wcost -0.3 and Wcost0.9 lines. The results confirm the common intuition that control by adjusting cost will have more effect if individuals are more sensitive about costs.

\subsubsection{Sensitivity to interaction}

The values of the parameters used to demonstrate interaction sensitivity were set to the same level as before:

$$
\begin{aligned}
& \beta^{1}=1, \\
& \beta^{2}=-0.6, \\
& \mu=6.6,
\end{aligned}
$$

with preference set: $\bar{X}^{1^{\prime}}=\{10,10,3,3,3\}$,

equal cost scenario: $\bar{X}^{2}=\{10,10,10,10,10\}$,

unequal cost scenario: $\bar{X}^{2}=\{10,10,5,5,5\}$.

We have three interaction impact levels for the interaction parameter $(\theta)$, respectively $0,-0.02$ and -0.1 . Each interaction level has a pair of results under different cost settings. In Figure 4-2, the continuous lines show the results of including cost differences, while the dashed lines show the results with constant cost across 5 start time 


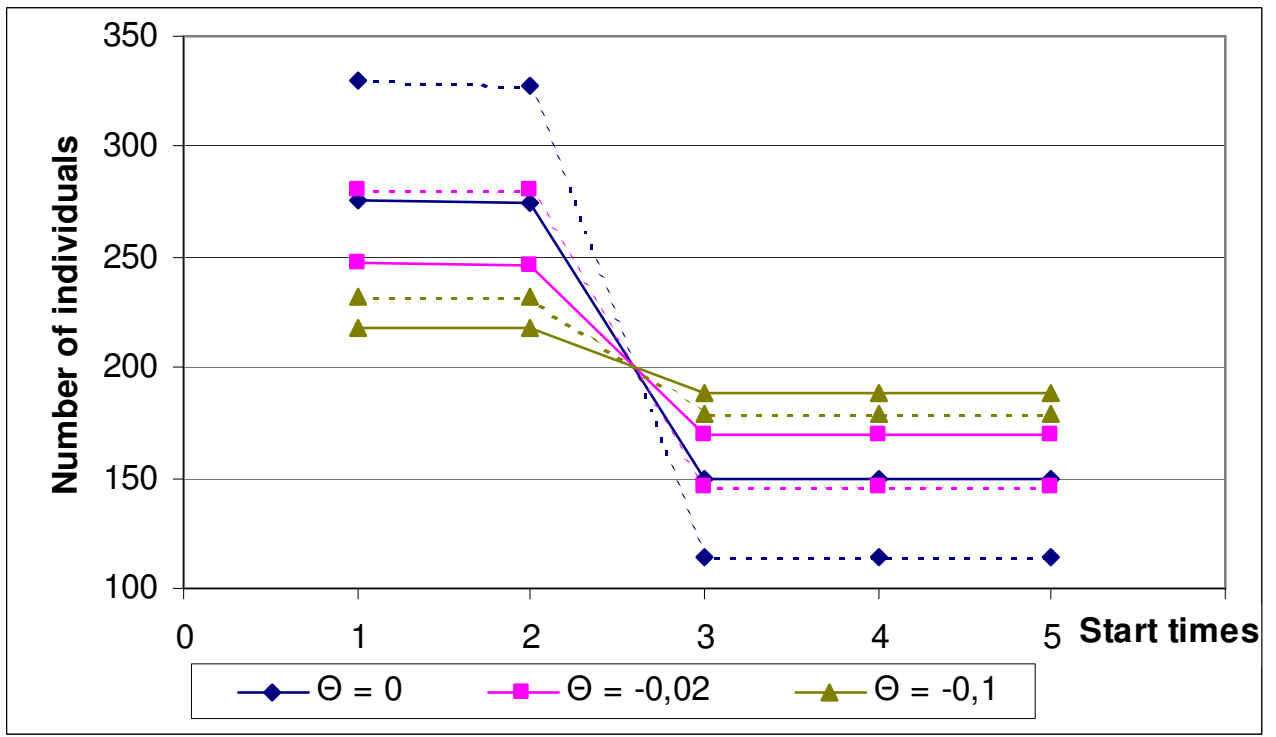

Figure 4-2 Interaction impact $(\theta)$

slots. It shows that if individuals do take interaction impact into account, they start to behave strategically. They begin to deviate from their originally preferred time, trying to avoid congestion to gain more utility. The more negative they feel about encountering other individuals, the more they deviate from their originally preferred start time. And this effect is additive to the cost sensitivity. That is, individuals will more likely choose these alternatives if the costs of starting at less preferred time slots are less.

\subsubsection{Sensitivity to uncertainty of conjectures}

The arbitrarily chosen values for parameters used for the uncertainty sensitivity test were:

$$
\begin{aligned}
& \beta^{1}=1, \\
& \beta^{2}=-0.6, \\
& \theta=-0.02, \\
& \bar{X}^{1}=\{10,10,3,3,3\}, \\
& \bar{X}^{2}=\{10,10,10,10,10\} .
\end{aligned}
$$

The model was tested using the estimated $\mu$ results from Goeree and Holt (2004). Since it is not possible for $\mu$ to take on a value of exactly zero in the simulation, which would be required for the Nash equilibrium, a low value of $\mu=1.0$ was used to approximate the distribution of the Nash equilibrium, implying that the best strategy is played with a probability close to 1 . The introspection probabilities were calculated by 


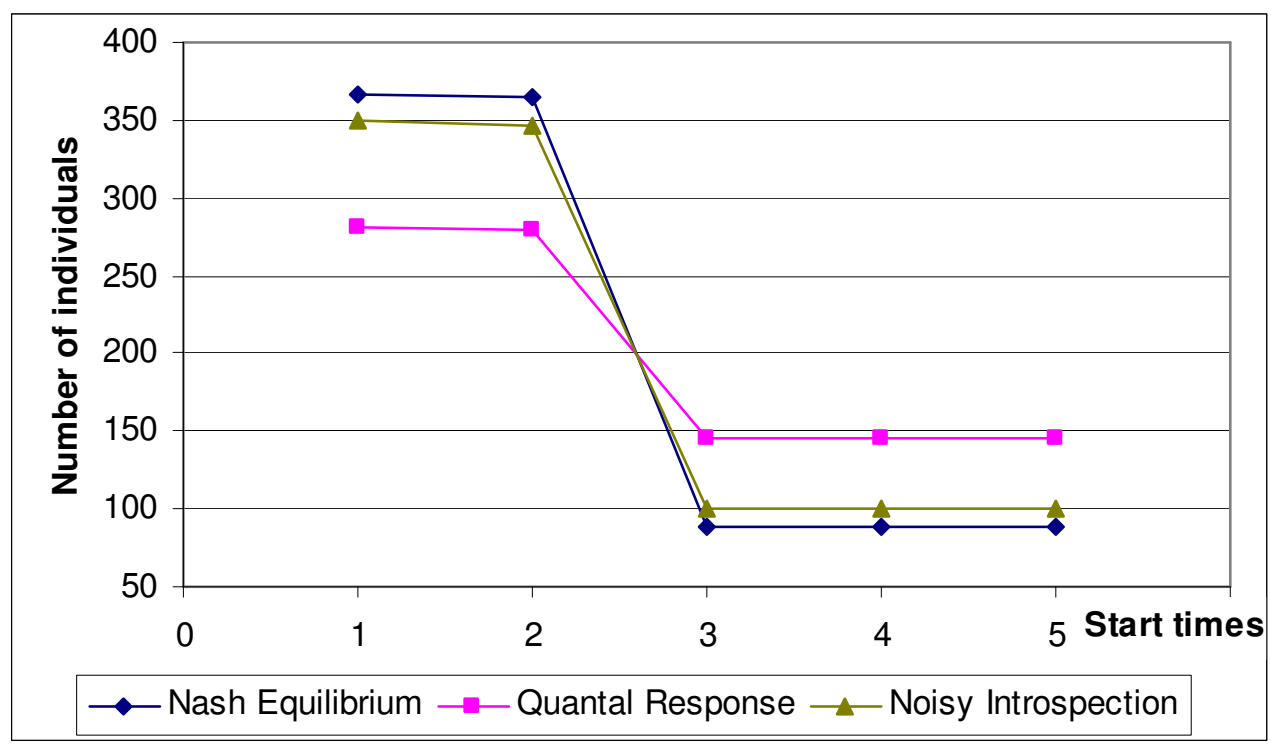

Figure 4-3 Uncertainty impact

taking the limit of the composition of functions on the right side of (3.9) as the number of iterations asymptotically approaches infinity. This was approximated by taking the first order error rate at $\mu_{0}=4.4$ and the noise geometric increase rate $t=4.1$ for equation (3.12) and truncating the right side of equation (3.9) at 5 iterations (i.e., the iteration reasoning order $k=5$ ), because of the fact that these probabilities are the same for every number of iteration reasoning order $(k)$ that is greater than 5 for the chosen $t$ value. The value $\mu=6.6$ (equivalent to $\mu_{0}=6.6$ and $t=1.0$ ) was used to indicate the quantal response equilibrium proposed by McKelvey and Palfrey (1995), which has the property that each of these probabilities is a logit stochastic response to the others.

As shown in Figure 4-3, the qualitative comparisons are consistent with common intuition. The Nash prediction stays closest to the timing preference since there are multiple effects involved. The fact that there is no uncertain information implies that the best strategies are chosen with a high probability, while the magnitude of $\mu$ works in the reverse direction. Introspection predictions are generally more suitable to reflect timing preference when individuals have no chance to learn what to expect, except for iterated guesses with increasing errors. When individuals have some idea about what others will do, they can gain more strategic advantage from starting at different times, implying that the quantal response distribution is more balanced. This offers a new avenue in that if individuals receive valid information about what other individuals are expected to do, which is similar to what they would receive through learning by own experience, then their behaviour can be influenced positively in the sense that they might act more in line with the objective of the information provider.

\subsection{Interactive decisions between individuals and an information provider}

The simulation was extended to test our strategic choice model of interactive decisions between individuals and an information provider. Assume a facility is open for 3 hours 
(every one hour interval is set as one start time slot), denoted by start time 1, 2, 3, and assume that the average visiting duration is one hour. In order to focus on aspects of individuals' interaction with the information provider, carry-over effects were left out, which means that individuals only take into account the impact of immediate encounters. For an individual who wishes to start visiting at time 2, the immediate encounters are only those visitors that start at the same time slot.

In this illustration, only start time preference was included in the choice alternative component of the model, which can also be taken as a composite of exogenous influences. The start time preference is the attractiveness of performing the activity during that time slot. For the interaction component among individuals, only one level effect was considered. For simplicity, it was assumed that disutility is linear in the number of individuals that have chosen the same start time. This implies that the marginal rate of an additional individual is constant. Based on equation (3.1), the simplified systematic self-expected payoff function that is used in the illustration, $E\left[\bar{U}_{2}\right]$, is given by:

$$
E\left[\bar{U}_{2}\right]=U_{2}^{\text {exogenous }}+\theta\left((N-1) p_{2}^{*}+1\right)
$$

The equilibrium information provision and participation pattern is then determined by integrating the collective individuals' trade-off between self-expectation and recommendation into the information provider's objective function to search for the optimum.

We conducted a set of numerical simulations to explore the combined effects of two factors. One factor concerns testing three different levels of individuals' conjecture abilities: (1) expectation with complete information in ideal situations - Nash equilibrium, (2) expectation with incomplete information in repeated situations - quantal response equilibrium, and (3) expectation with incomplete information in one-shot situations - noisy introspection. The second factor concerns three different objectives that an information provider may have: (1) equal usage: equal distribution across time slots, (2) welfare maximum: maximum utility across all individuals, (3) user equity: same utility for all individuals. The specific settings are explained along with the algorithm.

\subsubsection{Algorithm}

The participation and start time choice under recommendation was simulated for 1000 individuals, given an assumed preference vector for each start time and the parameters of the model. The following procedure was adopted in the simulations:

1. Draw 1000 sets of random values for the vector of preference from a normal density distribution with means (-3,0,3 for time slot 1,2, 3 respectively) and a variance equal to 1 . Every set defines an individual.

2. Use these values along with a marginal disutility of congestion (-0.006 per individual) to calculate the probability that an individual will choose a certain time slot, using equation (3.8). In this probability calculation, different values of $\mu$ are used to represent different levels of conjecture abilities: $\mu=1.0$ to approximate 
the Nash equilibrium, $\mu=6.6$ for the quantal response equilibrium, and $\mu_{0}=4.4$ and $t=4.1$ for the noisy introspection model, truncated at 5 iterations.

3. Sum the probabilities for each start time across all 1000 simulated individuals, generating a distribution of choices for three start times without recommendation (scenario A).

4. Use these self-expected probabilities and the information provider's recommendation to deduce the updated probabilities using equation (3.14) and calculate the compliance rate using equation (3.15).

5. Sum the probabilities for each start time across all 1000 simulated individuals, generating a distribution of choices for three start times with recommendation (scenario B).

6. Use these numbers to assess to what extent the information provider's objective has been achieved using equation (3.16) for the equal usage objective (with $\alpha_{1}=\alpha_{2}=1$, meaning that deviations from the reference level count the same for the information provider), equation (3.17) for the welfare maximum objective, and equation (3.18) for the user equity objective, and adjust the recommendation with a hill-climbing method.

\subsubsection{Simulation results}

The resulting equilibrium distribution across different start times and the corresponding recommendations represent the probabilities of starting the activity at a particular time slot rather than the exact start time choice frequencies. In general, it is assumed that an individuals' type is reflected in its time dependent preference and has a consequent recommendation perception error. In this simulation test, the combination effects of two factors were tested. In scenario A, no recommendation is provided, while in scenario B there is guidance information. By comparing the distribution of these two scenarios, an attempt was made to get some insight into the difference of providing information in driving individual decision-making and the consequences for achieving the information provider's objectives.

In order to compare the performance of the model for different objectives, the following measure was used. Each objective comes with a unique ideal distribution across different time slots. With this ideal distribution, the information provider has full control and his objective is perfectly achieved. This ideal distribution can be reached by replacing the compliance rate calculation in step 4 of equation (3.15) with all individuals completely following the recommendation (i.e., 100\% compliance rate). By measuring differences between the ideal distribution $\left(N P_{j}^{\text {ideal }}\right)$ and the resulting distribution based on the behavioural model ( $N P_{j}^{\text {behaviour }}$ ) for the same objective, a performance score for the information provider can be derived. This equation was specified as follows:

$$
Y=\sum_{j=1}^{j=3} \frac{\left(N P_{j}^{\text {behaviour }}-N P_{j}^{\text {ideal }}\right)^{2}}{N P_{j}^{\text {ideal }}}
$$


The smaller this score, the better the match. That is, the information provider's best performance is to obtain a score of 0 , if possible. Thus, the differences between scores obtained for scenario A (no recommendation) and scores obtained for scenario B (with recommendation) reflect improvements in how well the information provider's objective is achieved by providing guidance to influence individuals' decision-making. Theoretically, the range of performance scores is different, and depends on the nature of the objective.

Two analyses were conducted. First, the results were compared for different objectives of the information provider with the same level of individuals' conjecture ability. Second, the results were compared for different levels of individuals' conjecture abilities with the same objective for the information provider. The first analysis focuses on whether different objectives lead to differences in the generated advice and individual choice distributions. In contrast, the second analysis addresses the question whether different levels of conjecture abilities of individuals affect the achievement of the information provider's objective.

The results of the first analysis are portrayed in Figures 4-4, 4-5 and 4-6. The results of the second analysis are shown in Figures 4-7, 4-8 and 4-9. In these figures, the X-axis represents the objective of the information provider or the level of conjecture ability of individuals; the Y-axis on the left side indicates the number of individuals choosing a particular time slot, whereas the Y-axis on the right side expresses the information provided by the control individual about the population's timing choice probabilities. The bars portray the aggregated number of individuals at each time slot (coloured dark grey for the "without guidance" case, and coloured light grey for the "with guidance" case), while the lines show the provided advice information about timing choice probabilities. The tables show the performance scores for two scenarios with the optimum value of 0 .

\subsubsection{Effects of different objectives of an information provider}

Figure 4-4 shows the result of the Nash equilibrium. This equilibrium has the property that the best strategy is played with a probability that is close to 1 . In general, it reveals that recommendations differ for different objectives. In case of a recommendation based

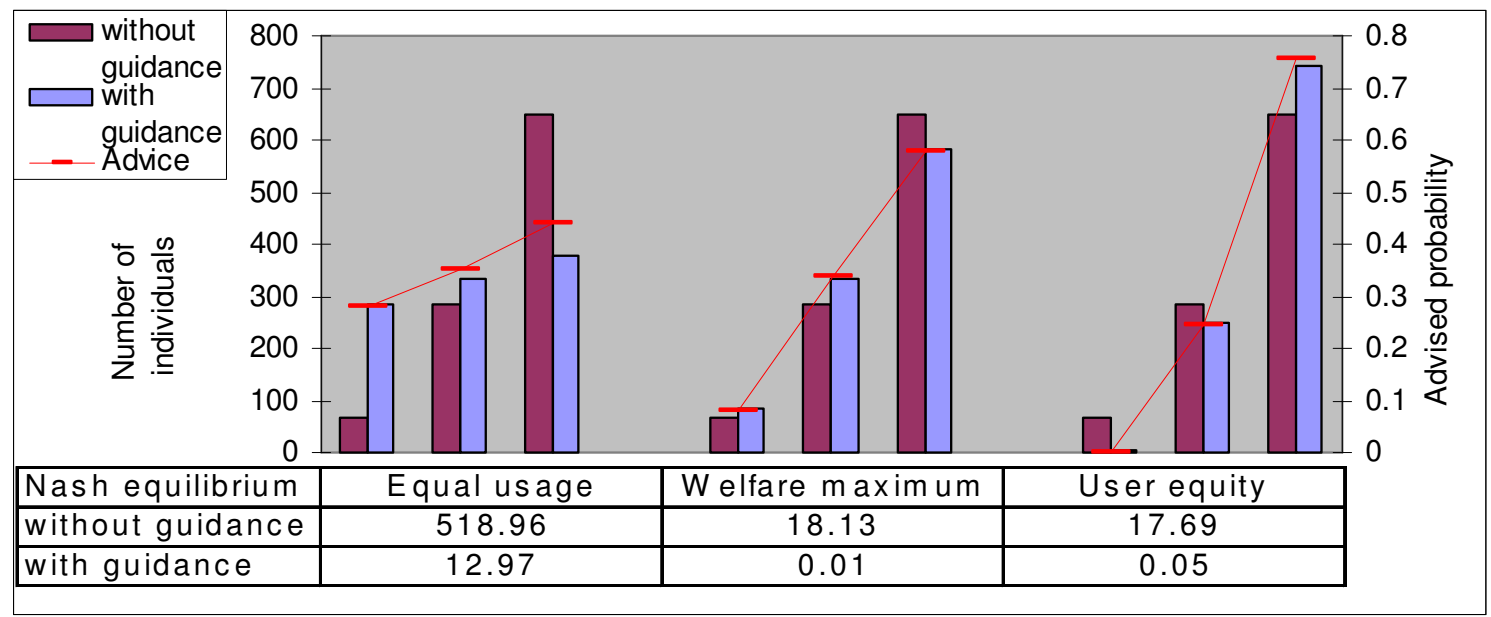

Figure 4-4 Results for the Nash equilibrium 
on the objective of equal usage, the most recommended option is time 1 , time 2 is also encouraged, but time 3 is discouraged. When welfare maximum is the objective, time 1 and time 2 are slightly recommended, but time 3 is not. It is reversed for the user equity objective, promoting more individuals to use time 3 and fewer individuals to choose time 1 and time 2.

Further, Figure 4-4 shows that recommendations have a clear effect on the choices that individuals make in the model. In case of a recommendation based on the equal usage objective, the choice of the initially most popular time 3 considerably decreases, while the initially less popular times 1 and 2 are chosen more often. This suggests that better results are achieved in terms of the information provider's objective. The effects of information provision seem less effective when welfare maximum is the objective of the information provider. When user equity is the information provider's objective, recommendations turn out to have an adverse effect: more individuals choose their most preferred time since the recommendation encourages them to do so. The reason for this effect could be that the initial settings for timing preference are too strong to be offset by the disutility brought along with congestion caused by the presence of additional individuals.

Figure 4-5 shows the result of the quantal response equilibrium. This equilibrium has the property that each individual's probabilities are a logit stochastic response to the others, which has the potential tendency to push the probabilities to a more equal distribution. Recommendation under the equal usage objective also results in a positive recommendation for time 1 and time 2, albeit more moderate. To offset the potential equal distribution, the recommendation under the welfare maximum objective encourages more individuals to choose time 3, opposite to the Nash equilibrium case. The recommendation under the user equity objective shows a similar propensity as that observed in the Nash equilibrium, encouraging time 3 and discouraging time 1 but the recommendation is more extreme.

The consequent individual distribution with guidance shows obvious differences from the distribution without guidance, reflecting the apparent impact of recommendation provision. Recommendation provision under the equal usage objective continues showing the positive effect that individuals distribute more evenly across time, but the effect becomes less compared with the Nash equilibrium. It has the same effect for the welfare

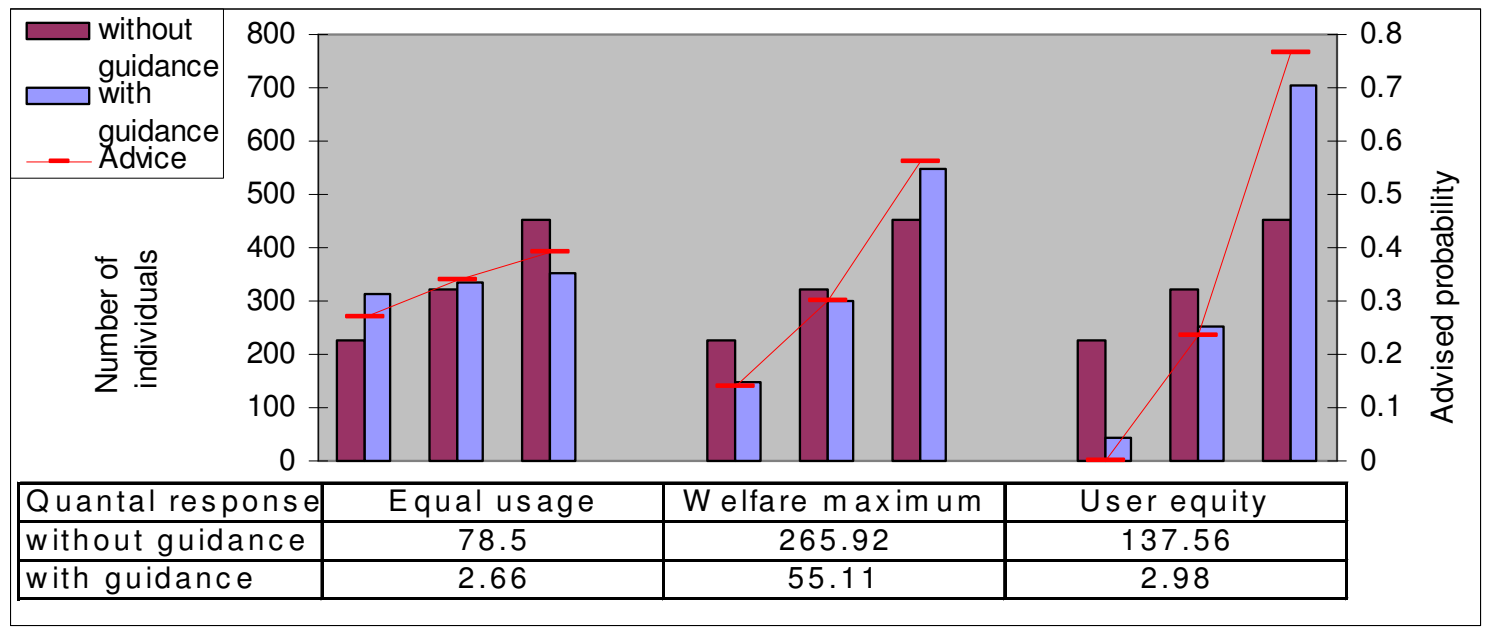

Figure 4-5 Results for the quantal response equilibrium 


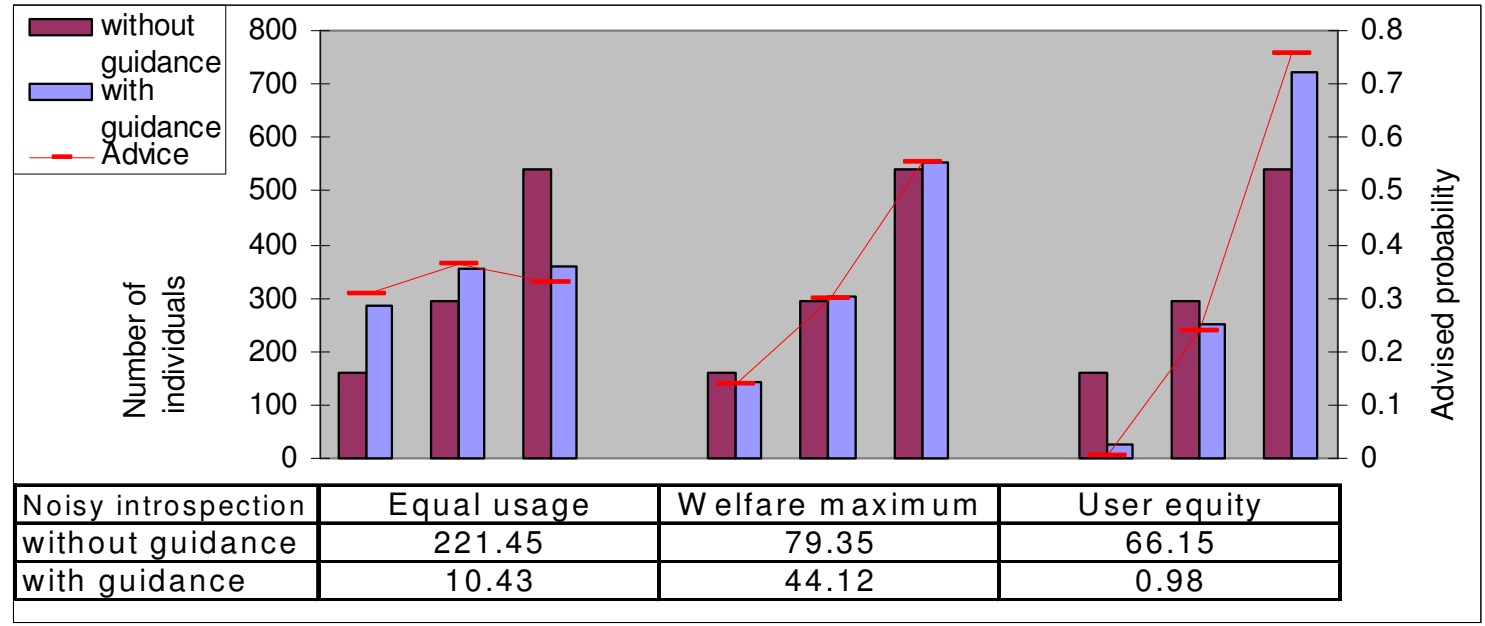

Figure 4-6 Results for the noisy introspection model

maximum objective, while it becomes more noticeable under the user equity objective.

The result for the noisy introspection is shown in Figure 4-6. This case is not an equilibrium solution but rather an out-of-equilibrium situation, which has the property of an iterated quantal response function with a response error parameter increasing in an iterative reasoning process to arrive at some choice. The similar propensities as observed in the equilibrium cases also apply to the noisy introspection case. The noticeable difference is the recommendation for the equal usage objective. In this case, time 3 is discouraged more, since individuals are expected by the information provider to more easily follow the recommendation when they have more error in their reasoning caused by incomplete information and no experience in one-shot situations.

From the results for the different objectives under the same level of expectation ability among individuals, we can see that recommendations are relatively less effective in influencing individuals' decisions when the information provider intends to maximize welfare and achieve user equity. A possible reason for this difference in effectiveness is that recommendations are less influential when the information provider's objective is more or less the same as that of the individuals. With the recommendations being fairly similar to individuals' self-expectations, the outcomes will at best show subtle changes. In the case of pursuing equal usage of the facility over time, the individuals' objective is far removed from the information provider's objective and recommendations are therefore more influential.

The extent to which the recommendation can differ from the individuals' selfexpectations and still be effective, however, is limited due to potential shifts in individuals' compliance rates, and the consequent outcome is, therefore, always a compromise. The general tendency underlying these results is that different objectives bring different recommendations and consequently have distinctive individual distributions. 


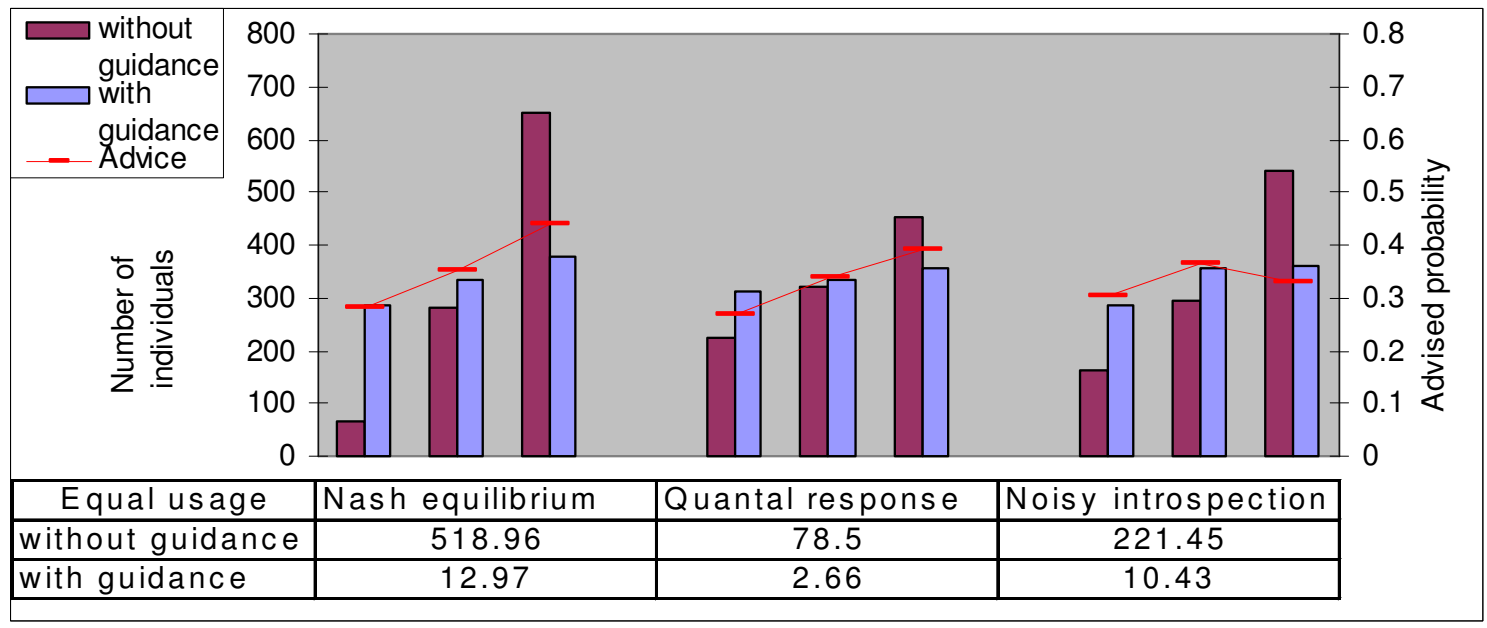

Figure 4-7 Results for the equal usage objective

\subsubsection{Effects of different levels of individuals' conjecture ability}

To make the effect of different levels of conjecture abilities among individuals more obvious, we rearranged the results on the basis of the same objective in the following three figures

Figure 4-7 shows the results for equal usage as the information provider's objective. The setting, without guidance, with a quantal response equilibrium brings the information provider the highest score, while the Nash equilibrium has the lowest score. The quantal response equilibrium implies individuals stochastic response to each other, which has the potential tendency to push the probability to a more equal distribution. An equal distribution is the essential focus of the equal usage objective. With guidance, the same pattern is still valid, although the Nash equilibrium shows a great improvement.

Figure 4-8 shows the results for welfare maximum as the information provider's objective. The setting, without guidance, with the Nash equilibrium that individual's

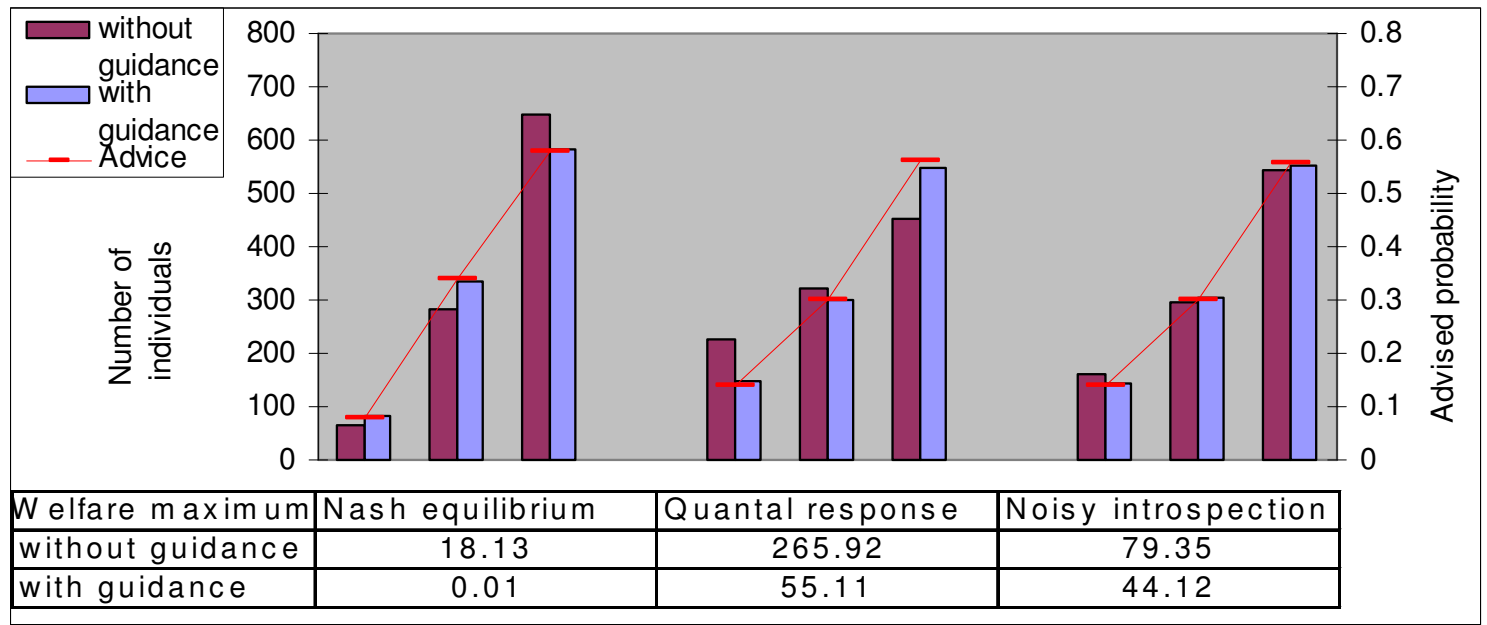

Figure 4-8 Results for the welfare maximum objective 


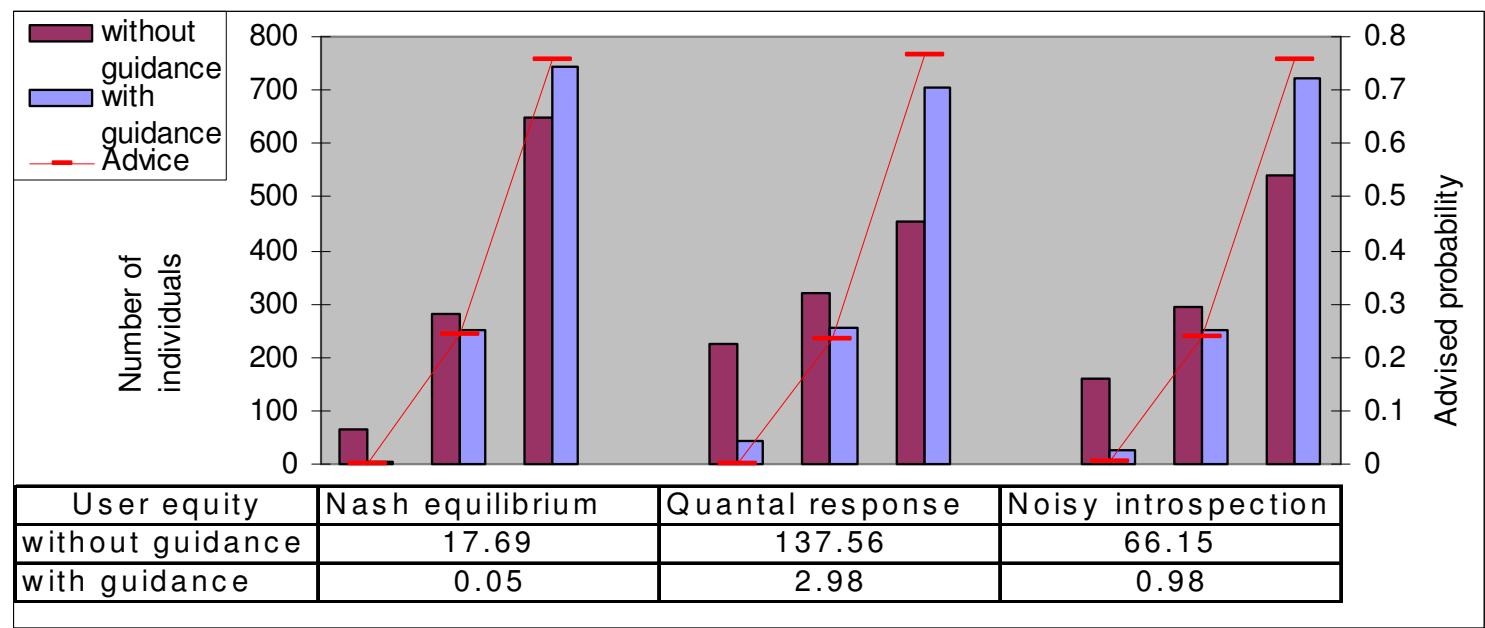

Figure 4-9 Results for the user equity objective

optimal response to each other with high certainty brings the information provider the highest score, while the quantal response equilibrium has the lowest score. The Nash equilibrium implies individuals choose the best strategy with a probability close to 1 , while the objective of welfare maximum implies one individual's welfare could be compensated by another's, which is somewhat in line with individuals' personal best interest. With guidance, the Nash equilibrium almost reaches the highest possible score, while the quantal response shows a great improvement but still gets the lowest score. The noisy introspection reflects less improvement compared to the quantal response.

Figure 4-9 shows the results for user equity as the information provider's objective. In this setting without guidance, the Nash equilibrium brings the information provider the highest score, while the quantal response equilibrium has the lowest score. With guidance, the Nash equilibrium almost reaches the highest possible score, while the quantal response still has the lowest score.

Comparing the results of the two equilibriums, the Nash equilibrium seems to perform better in both the welfare maximum and user equity case. But with equal usage level as the objective, the Nash equilibrium receives the lowest score. This difference is due to the assumption that in the Nash equilibrium the individual's best strategy is chosen with certainty, which implies the least willingness to adjust behaviour, especially when the objective underlying the recommendations is not in line with the interest of individuals.

The results for the noisy introspection, in contrast for the Nash equilibrium, induced a good performance for the equal usage objective, but are worse for the welfare maximum objective. Compared with the quantal response equilibrium, the noisy introspection shows more improvement in case of the equal usage objective because individuals are easier to follow the recommendation when their reasoning between beliefs and actions is inconsistent.

Generally, when individuals are less certain about what others will do, they show more willingness to follow the recommendations as in the noisy introspection situations, leading to a greater improvement in terms of the information provider's objective. Thus, the information provider may need to provide slightly different recommendations, and 
may need to assess the best possible achievement differently, if reality involves uncertainty about others' behaviour.

Above all, the simulation results illustrate that providing recommendations can influence individuals' behaviour and assist in achieving the information provider's objective. But the extent to which recommendations affect the individuals' choices depends on the information provider's objective. At the same time, the possible achievement of an information provider's objective needs to be assessed on the basis of the levels of conjecture abilities among individuals, which depends on context.

\subsection{Conclusion and discussion}

This chapter has described the results of numerical simulations to explore the property of our model of strategic choice behaviour within a specific context. The results of the numerical simulation provided a positive answer to our research question. The proposed model is able to produce distinguishable choice behaviour when employing different parameter values, confirming its sensitivity to different parameter settings. Furthermore, the different results are easy to interpret in behavioural terms and consistent with intuitive expectations, supporting the face validity of the model.

The results of the numerical simulations suggest that the proposed approach is capable of representing the assumed interaction patterns both between individuals at the disaggregate level and between an information provider and individuals at the aggregate level. Moreover, the simulation results are consistent with one's expectations. Hence, it seems that the developed model is a potentially valuable tool for information providers to more effectively and efficiently achieve their objectives by choosing optimal guidance strategies while taking into account the reaction of individuals.

The simulation suggests that the endogenously determined interaction may influence the distribution of individual's choices as well as the exogenous variables. Different levels of conjecture abilities underlying the decisions of individuals bring about an apparently different distribution. These distributions could be further transformed as a result of responses to the recommendations provided with the various objectives. The study indicates that differences of these recommendation effects vary considerably with the information providers' objective.

In conclusion, the results support the face validity of the proposed model. Although this study has taken the choice of visiting a facility as an example, the modelling approach could also be used to model other individuals' decisions under guidance. In the next chapter, we will use tailored experiments to collect empirical data, estimate and validate the formulated model. 


\section{Chapter 5}

\section{Timing Choice}

\subsection{Introduction}

In the previous chapter, we have reported the results of a series of numerical simulations, which were conducted to assess the sensitivity of the various parameters of the strategic choice behaviour models that we developed. These results of these simulations supported the face validity of the formulated models.

In this chapter, we report the results of an experiment that was conducted to empirically test how individuals incorporate strategic considerations with respect to congestion in their timing decisions in one-shot situations. Because we did not have a specific field of application in mind, but rather are interested in model types that potentially could be further elaborated and applied to many different problems of strategic consumer choice behaviour under conditions of uncertainty, the application domain and the specific choice task were varied across two different contexts: (1) a hypothetical choice of dinner time while on vacation in London ("dinner timing choice"), and (2) a hypothetical and real-world choice of participation time for a research experiment ("participation timing choice").

The chapter is organized as follows. First, the hypothesis concerning the nature of individual strategic choice behaviour in one-shot situations is discussed. Next, the design of the experiment as well as the data collection is presented. Then, the methods of 
analysis and the results are discussed. Finally, a conclusion and discussion section finishes this chapter.

\subsection{Hypothesis}

The basic assumptions that underlie our theory and models are that decision makers pursue well-defined exogenous objectives (they are rational) and take into account their expectations of other decision makers' behaviour (they reason strategically). More specifically, we assume that congestion in capacity-constrained services is essentially the negative outcome of the aggregation of individual-level choices that individuals make in self-interest. Choices made by individuals lead to aggregate, macro-level service consumption patterns, which in turn may influence individual, micro-level, decisions. The equilibrium consumption timing pattern is then determined by the trade-off between timing preference and anticipation of congestion. In other words, individuals' anticipation of other individuals' choices for an activity participation time affects their own preferences for different participation times. To anticipate other individuals' activity participation time choices, each individual needs to take into account not only the other individuals' timing preferences, but also how these individuals respond to potential congestion and adjust their choices accordingly.

The proposed models are based on a game structure and assumptions about payoff functions. In particular, the assumptions about preferences of individuals are: (1) individuals have similar preferences for activity participation times and dislike congestion; (2) individuals have different distributions of preferred participation times for different activities. The assumptions regarding the reasoning of individuals about the behaviour of other individuals are: (1) individuals hold beliefs about originally preferred activity times of other individuals and anticipate the trade-off between originally preferred time and congestion of other individuals; (2) individuals can adjust their strategic anticipation of congestion through information and/or experience.

As described in Chapter 3, the conventional Nash equilibrium model implies a deterministic model with perfectly rational behaviour in which the optimal strategy is chosen with certainty. The more general statistical theory underlying the quantal response equilibrium is based on the notion that better strategies are more likely to be played than worse strategies, but best strategies may not always be played, due to various sources of error in individual's evaluations of the different strategies in the game (such as cognitive limitations, or incomplete information about other individuals' preferences.)

Consistent with this line of interpretation in empirical game theory, research in static decision-making has also shown that in complex choice situations individuals' utilities are not deterministically related to the choices they make (e.g., Swait and Adamowicz, 2001). Therefore, in our empirical tests, we expect a similar effect to occur in individuals' choices of participation times for capacity-constrained services. In particular, we expect that individuals' ability to take into account other individuals' choices is limited, leading to deviations from the Nash equilibrium and causing individuals' anticipation of other individuals' participation time choices for capacityconstrained services to contain errors. Thus, we expect individuals' choices of participation times with anticipated congestion to be consistent with a quantal response equilibrium rather than a Nash equilibrium. 
Moreover, when such strategic choice is only made once, individuals do not have the opportunity to observe and get familiar with the behaviour of other individuals, and therefore we expect that individuals' ability to conjecture other individuals' choices is restricted to iterative reasoning with increasing errors; that is, out-of-equilibrium. In summary, in one-shot strategic situations with anticipated congestion, we expect individuals' participation time choices to be closer to the noisy introspection model rather than to a quantal response equilibrium. Therefore, the following hypothesis is tested:

Hypothesis: In one-shot strategic situations, a quantal response model leads to more precise predictions of strategic (participation timing) choice behaviour compared to a Nash equilibrium model, but a model of noisy introspection predicts even better.

In order to judge whether the developed approach can be used successfully to describe and understand strategic choice processes, all assumptions should be tested. Whereas testing the assumptions is rather straightforward, testing the hypotheses about conjecture ability requires more advanced means of analysis.

\subsection{Timing choice data}

Testing the proposed approach from real-world service consumption timing choices can be problematic because typically the anticipated inter-personal effects are confounded with (unobserved) personal constraints such as time budgets, scheduling obligations, and so on. Therefore, the model estimation results are typically difficult to interpret. For this reason, we conducted a controlled experiment to verify whether the assumptions underlying the models are valid.

\subsubsection{Data collection}

Data for the study were collected in 2003-2004 over a number of sessions. Participants were first year undergraduate students in the Department of Economic Psychology at Tilburg University, who participated for course credit. A total of 118 participants registered and participated. The experiments consisted largely of a paper and pencil task

Table 5-1 Overview of registration for timing choice experiment

\begin{tabular}{|c|c|c|c|c|c|}
\hline & $31-03-2003$ & $22-05-2003$ & $12-11-2003$ & $05-02-2004$ & Total \\
\hline $2: 30 \mathrm{pm}$ & 3 & 14 & 6 & 61 & 84 \\
\hline $4: 00 \mathrm{pm}$ & 1 & 8 & 3 & 7 & 19 \\
\hline $5: 30 \mathrm{pm}$ & 6 & 3 & 4 & 2 & 118 \\
\hline Total & 10 & 25 & 13 & 70 & 15 \\
\hline
\end{tabular}


and participants took approximately 30 minutes to finish the task. Participants were recruited in two ways: (1) By putting an announcement on the board, and registering via email; (2) By sending the announcement through the email list, and registering by replying. Table 5-1 shows the overview of the registration.

The experiment involved multiple tasks, covering two application contexts: (1) a hypothetical choice of dinner time while on vacation in London ("dinner timing choice"), and (2) a hypothetical and real-world choice of participation time for a research experiment ("participation timing choice"). All subjects responded to several choices in both contexts. The specific scenarios subjects faced within each context differed, depending on the manipulation in the experimental task that was presented to the subject. The details of these manipulations are explained in the following sections.

\subsubsection{Dinner timing choice}

The objective of the "dinner timing choice" section of the study was to investigate to what extent individuals take into account other individuals timing decisions when choosing their own service consumption time. In this context, subjects were asked to imagine that they were in London for a weekend with a friend and were planning on going out for dinner on Saturday night. Subjects were told they did not have other obligations that evening and that from their travel guide, they had selected a number of restaurants, none of which they had visited before. They were also told that these restaurants were all comparable in quality, ambience and price. Then, they were presented with a number of choice tasks in which they were asked to select the restaurant for which they would prefer to make a reservation. Subjects were asked to make a reservation in the restaurant choosing one of three dinner times, and were told that they might have to wait at the restaurant before they could start their dinner.

There were two versions of the "dinner timing choice" context that were randomly assigned to each subject. Approximately half of the subjects received scenarios that involved a strategic decision-making situation. In these scenarios, we emphasized that the reason that subjects might have to wait was that although each restaurant takes reservations, they assign guests arriving at the restaurant on a first come first serve basis. Thus, depending on the number of guests, on average all guests will have to wait longer for a table. The length of this waiting time is unknown before getting to the restaurant.

A $3^{2}$ full-factorial experimental design was used to generate nine alternative restaurant profiles. The use of a full-factorial design guaranteed that all main effects could be estimated independently from potential interaction effects. The manipulated factors were: (1) reservation time at the restaurant $(5: 00 \mathrm{pm}, 7: 30 \mathrm{pm}$ or $10: 00 \mathrm{pm})$ and (2) booking policy of the restaurant in terms of the number of guests taken relative to capacity ("close to capacity", "at full capacity", or "more than capacity"). The latter was included to control for possible assumptions that subjects might have regarding the booking policy of the restaurant and that might affect their congestion anticipations. Each subject received three choice sets that each consisted of three profiles from the design (randomly combined without replacement), and a "none of these" option as a base alternative.

The second half of the subjects received a baseline comparison version that involved a non-strategic dinner timing choice. In this scenario, the reason that subjects 
might have to wait was formulated as follows: although all restaurants allow guests to book a dinner time in advance, the specific table that you booked may not be available immediately. This waiting time is driven by random variation in the time the previous guests at the table decide to finish their dinner. The restaurant does, however, provide you with information on the expected waiting time and with an indication of the uncertainty of this wait. In this version, a $3^{3}$ full-factorial experimental design was used to generate 27 profiles. The full-factorial design guaranteed that all main effects could be estimated independently from potential interaction effects. The manipulated factors were: (1) reservation time at the restaurant $(5: 00 \mathrm{pm}, 7: 30 \mathrm{pm}$ or 10:00 pm), (2) expected waiting time $(0,10$ or 20 minutes), and (3) the probability of a 10 minutes additional delay $(0 \%$, $20 \%$ or $40 \%$ probability). The latter two factors controlled for subjects' possible assumptions regarding waiting time in the restaurant. In this version, each subject received nine choice sets that each consisted of three profiles from the design (randomly combined without replacement), and a "none of these" option as a base alternative.

\subsubsection{Participation timing choice}

The objective of the "participation timing choice" section of the experiment was: (1) to investigate individuals' consumption timing choices in a real-world setting involving potential congestion, (2) to compare the effect of the strategic anticipation of other individuals' behaviour as well as the non-strategic effects with the study of "dinner timing choice", and (3) to obtain measures of individuals' non-strategic consumption timing choices in the same setting and to compare: (a) individuals' real-world timing choices, (b) their choices based on the noisy introspection model, (c) their choices based on a quantal response equilibrium, and (d) their choices based on a Nash equilibrium. The three objectives were met by distinct parts of the experiment.

To investigate the first objective, we used subjects' actual registration times for our experiment. Potential subjects for the experiment were recruited so as to guarantee that they had no information about attendance levels per session that was available, except for the fact that congestion might occur. This was done by placing an announcement on the board asking subjects to register via email, and by sending an announcement through a student email list, asking subjects to register by email-reply. In the announcements, three sessions were presented for each day in which subjects could participate in the experiment (session time slots were 2:30 pm, 4:00 pm or 5:30 pm). Subjects were free to choose one of these sessions for their participation time, but were notified in the announcement that capacity was limited for each session and that they might have to wait. Thus, subjects had to anticipate whether or not, and if so, to what extent congestion might occur at a given time. At the start of the experiment, subjects were also asked to report for each time slot the number of other students they expected would also participate at the same time and their anticipated waiting time. This data was later used as input in the timing distribution simulations and analysis (see Section 5.4).

For the second and third objective, hypothetical choice tasks regarding subjects' timing preference for participating in an experiment were used. The task represented a non-strategic choice involving expected waiting times, similar to the second version of the "dinner timing choice" task. This task measured subjects' non-strategic utility for time slots for participating in an experiment. Subjects were asked to choose a start time for a hypothetical experiment in a number of choice sets. Each subject was asked to imagine 
that they are going to register for participation in an experiment and can choose between several of registration time slots. They were also told that on the day of the experiment they did not have any other obligations, but they might have to wait some time after arriving at the location where the experiment is conducted. Subjects were told that the reason for this anticipated wait was that the experiment required an elaborate computer and laboratory set-up, and it was emphasized that they did not have to wait for others to complete the experiment.

A $3^{3}$ full-factorial experimental design was used to generate 27 profiles presented in the choice sets. The full-factorial design guaranteed that all main effects could be estimated independently from potential interaction effects. The manipulated factors were: (1) start time $(2: 30 \mathrm{pm}, 4: 00 \mathrm{pm}$ or $5: 30 \mathrm{pm})$, (2) expected waiting time $(0,10$ or 20 minutes), and (3) probability of a 10 minutes additional delay on top of the expected waiting time $(0 \%, 20 \%$ or $40 \%)$. Each subject received nine choice sets, consisting of three profiles from the design (randomly combined without replacement) and a "none of these" option as a base alternative.

For the strategic choice, there was an additional choice task in the "participation timing choice" context which was designed to explore how participants' timing preference might be influenced by the specific information about the distribution of other individuals and to obtain a proxy of subjects' disutility for congestion in the experiment. In this task, subjects were asked to choose a start time for a potential follow-up experiment to take place in the near future. They were told that the next experiment would be run in the computer lab, and therefore that capacity was limited and they might have to wait before they could participate in a given time slot. They were informed that a total of 100 students was required for the experiment, that there would be three time slots, and that waiting would occur if the number of participants at any given time slot would be greater than 25 . They were also told how many students were expected to register at each time. A $3^{2}$ full-factorial experimental design was used to generate nine profiles. The manipulated factors were: (1) start time $(2: 30 \mathrm{pm}, 4: 00 \mathrm{pm}$ or 5:30 pm), and (2) the expected number of students that would register at that time (17, 33 or 50 subjects). Each subject received one randomly assigned choice set of three profiles and a "none of these" option as a base alternative. Choice sets were constructed such that a profile for each time slot occurred only once in the set, and the total number of expected students across the three time slots added up to 100 . Thus, seven choice sets were constructed that were as much as possible balanced for expected number of students over start times within the constraint of not exceeding the total number of expected students.

\subsubsection{Structure of the experimental design}

Figure 5-1 gives a graphical representation of the intended plan. Assumptions regarding the impact of congestion and preferred access time, are justified using 1-A and 1-B "dinner timing choice" data. With and without strategic thinking comparisons both for "dinner timing choice" (1-A \& 1-B) and "participation timing choice" (2-B \& 2-C) are used to verify conjectures of other's preference about timing choice. Across two contexts, comparing 1-A and 2-B, data analysis clarifies assumptions about the different timing preference between different activities. The difference in anticipation about other's tradeoff in different activities is tested using data (1-B) and (2-C). An estimation of strategic choices under information (1-C) sheds light on behavioural adjustment. With the answers 


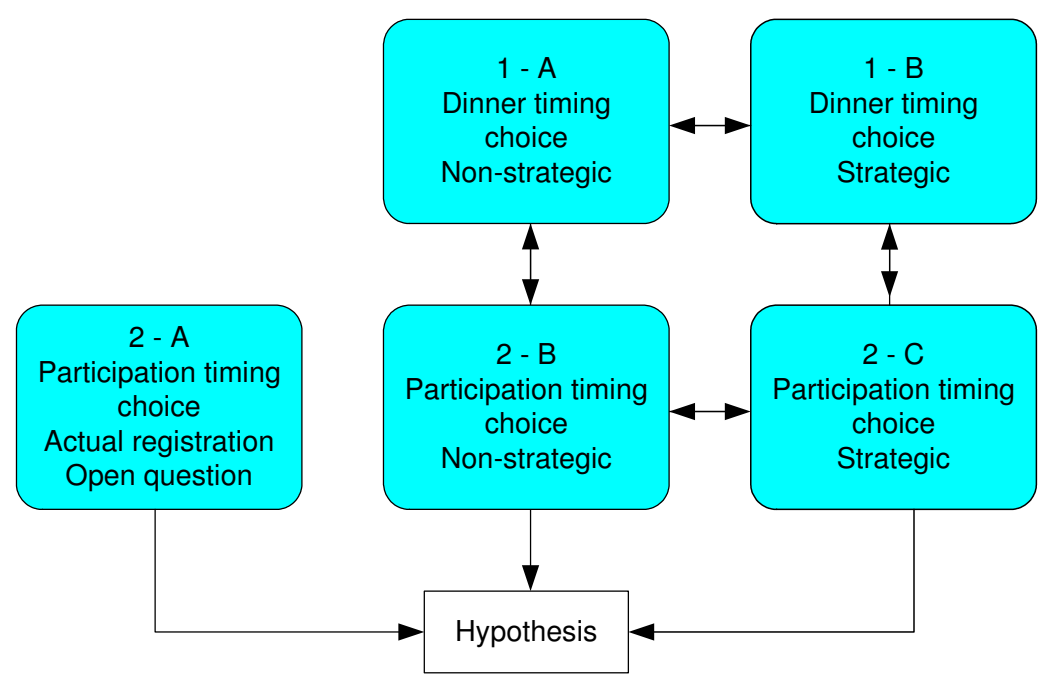

\section{Figure 5-4 Structure of timing choice experimental design}

to open questions and the actual participation timing choice registration (2-A), a set of model simulations predict the outcome of participation timing choices and provide the answer to the hypothesis. Appendix A-1 provides an example of the announcement, and Appendix A-2 shows an example of the questionnaire.

\subsection{Analysis of timing choice data}

In order to test the assumptions, in general, a multinomial logit (MNL) model and a random coefficients logit (RCL) model were estimated. We will give a detailed explanation of the method according to its special purpose and the results respectively.

\subsubsection{Dinner timing choice model}

In analyzing the preference for different times of undertaking an activity, we implemented a more general form of the MNL model - the RCL model that allows for heterogeneity in individuals' preferences for time slots (McFadden and Train, 2000). The model structure also captures the repeated measures nature of our data in which each subject responded to multiple choice sets. We first define the utility model for the nonstrategic dinner timing choice task:

$$
\begin{aligned}
& U_{i t}^{d i n N S}=\alpha_{i t}^{d i n N S}+\boldsymbol{\beta}_{\text {wait }}^{\text {dinNS }} \mathbf{X}_{\text {wait }}+\boldsymbol{\beta}_{\text {add }}^{\text {dinNS }} \mathbf{X}_{\text {addwait }}+\varepsilon_{i t k}^{d i n N S} \\
& \alpha_{i t}^{d i n N S}=\alpha^{d i n N S}+\left(\gamma_{\mathbf{t}}^{\text {dinNS }}+\mathbf{v}_{\mathbf{i t}}^{\text {dinNS }}\right) \mathbf{X}_{\mathbf{t}}
\end{aligned}
$$

where $U_{i t}^{d i n N S}$ is subject $i$ 's utility of dinner time slot $t$ in the non-strategic dinner choice task; $\alpha_{i t}^{d i n N S}$ is an individual and time slot specific random intercept, with average $\alpha^{d i n N S}$, 
Table 5-2 Non-strategic dinner timing choice estimates (1-A)

\begin{tabular}{|c|c|c|c|c|c|c|}
\hline & \multicolumn{2}{|c|}{ MNL model } & \multicolumn{4}{|c|}{ RCL model } \\
\hline Attribute & Parameter & t-value & Parameter & t-value & $\begin{array}{l}\text { Standard } \\
\text { deviations }\end{array}$ & t-value \\
\hline Time $-5: 00 \mathrm{pm}$ & $-0.45^{*}$ & -4.07 & $-1.03^{*}$ & -3.10 & $1.93^{*}$ & 5.73 \\
\hline Time $-7: 30 \mathrm{pm}$ & $2.13^{*}$ & 14.79 & $3.54^{*}$ & 8.17 & $2.05^{*}$ & 5.63 \\
\hline Waiting time & $-0.11^{*}$ & -11.19 & $-0.16^{*}$ & -10.71 & na & \\
\hline Probability of additional wait & $-0.03^{*}$ & -7.23 & $-0.04^{*}$ & -7.41 & na & \\
\hline Intercept & $3.76^{*}$ & 12.61 & $5.36^{*}$ & 10.98 & na & \\
\hline Log likelihood null model & \multicolumn{2}{|c|}{-711.17} & \multicolumn{4}{|c|}{-711.17} \\
\hline Log likelihood final model & \multicolumn{2}{|c|}{-421.10} & \multicolumn{4}{|c|}{-365.34} \\
\hline Adjusted Rho-square & \multicolumn{2}{|c|}{0.41} & \multicolumn{4}{|c|}{0.48} \\
\hline
\end{tabular}

* significant at $\mathrm{p}<.05$ level; na $=$ not applicable.

a time slot specific effect $\gamma_{t}^{\operatorname{din} N S} \mathbf{X}_{t}$, and a normally distributed individual and time specific random component $\boldsymbol{v}_{\mathrm{it}}^{\mathrm{din} N \mathrm{~S}} ; \boldsymbol{\beta}_{\text {wait }}^{\text {dinNS }} \mathbf{X}_{\text {wait }}$ and $\boldsymbol{\beta}_{\text {addwait }}^{\text {dinNS }} \mathbf{X}_{\text {addwait }}$ are the effects of waiting and the probability of an additional wait respectively in the non-strategic choice task, and $\varepsilon_{i t k}^{d i n N S}$ is an IID Gumbel distributed error term that is individual, time and observation $(k)$ specific.

Table 5-2 presents the parameter estimates for the designed experiment of restaurant visits without strategic consideration. The number of choice sets is 513 , and the number of observations is 2052 . The number of parameters to be estimated is five and seven for the MNL model and the RPL model respectively. The overall fit of the model is good, with an adjusted pseudo Rho-squared value around 0.4. All of the parameter values are significant at the $95 \%$ confidence level. The test statistics show that the models outperform the model with all parameter equal to zero (null mode). It also shows that the RCL model outperforms the MNL model according to the log likelihood ratio test at the 95\% significance level. This result is consistent with our intuition that the waiting time and uncertainty about additional wait bring a negative impact when people assess the utility for making a decision. After correcting for the effect of waiting time and uncertainty, subjects show a clear preference for a dinner time at 7:30 pm. They do not really like to have dinner at 5:00 pm and 10:00 pm, but would rather go at 5:00 pm instead of 10:00 pm. Although they show some common trend for timing preferences to undertake the activity, they also show clear individual heterogeneity in timing preference.

The utility model for the strategic dinner timing choice task follows a similar structure: 


$$
\begin{aligned}
& U_{i t}^{d i n S}=\alpha_{i t}^{d i n S}+\boldsymbol{\beta}_{\mathbf{p o l}}^{\mathrm{dinS}} \mathbf{X}_{\mathbf{p o l}}+\varepsilon_{i t k}^{d i n S} \\
& \alpha_{i t}^{d i n S}=\alpha^{d i n S}+\left(\boldsymbol{\gamma}_{\mathbf{t}}^{\mathrm{dinS}}+\mathbf{v}_{\mathbf{i t}}^{\mathrm{dinS}}\right) \mathbf{X}_{\mathbf{t}}
\end{aligned}
$$

where $\boldsymbol{\beta}_{\mathbf{p o l}}^{\mathrm{dinS}} \mathbf{X}_{\mathrm{pol}}$ is effect of the restaurant's capacity policy, and all other components are defined analogously to the non-strategic choice task.

In analyzing individuals' attitude towards information about congestion, how they take into account the relevant information about the capacity and time, we estimated an additional random coefficients logit model including the interaction effect of capacity policy with timing. In this model, a $3^{2}$ full-factorial recoding of capacity policy with timing was used to generate nine profiles for further investigating the interaction effect. The use of a full-factorial recoding guaranteed that all interaction effects could be estimated independently from the main effects of timing preference $(T)$ and capacity policy information $(C)$. When this interaction is significant, we conclude that the preferences are significantly affected by the interaction effect between time and information about capacity policy. The model estimated to perform this test is expressed as:

$$
\begin{aligned}
& U_{i t}^{d i n S}=\alpha_{i t}^{d i n S}+\boldsymbol{\beta}_{\mathbf{p o l}}^{\operatorname{dinS}} \mathbf{X}_{\mathbf{p o l}}+\varepsilon_{i t k}^{d i n S} \\
& \alpha_{i t}^{d i n S}=\alpha^{d i n S}+\left(\gamma_{\mathbf{t}}^{\operatorname{dinS}}+\gamma_{i n t}^{d i n S} \boldsymbol{X}_{i n t}+\boldsymbol{v}_{\mathrm{it}}^{\operatorname{dinS}}\right) \mathbf{X}_{\mathbf{t}}
\end{aligned}
$$

where $\gamma_{\text {int }}^{\text {dinS }} \boldsymbol{X}_{\text {int }} \mathbf{X}_{\mathrm{t}}$ is the interaction effect of capacity policy and time slot, and all other components are defined analogously to before.

Table 5-3 presents the parameter estimates for the designed experiment of restaurant visits with strategic consideration. The number of choice sets is 183 , and the number of observations is 732 . The number of parameters to be estimated is seven for the RCL model, and 11 for the RCL model with the interaction effect. It is clear that the information about booking policy of the restaurant at "close to capacity", "at full capacity", and "more than capacity" plays a role in the decision-making process. Obviously, booking at "more than capacity" has a negative influence, while booking at "close to capacity" has a positive effect. The difference of dinner time preference is mediated by the consideration of offered booking policy information.

After further investigation of the interaction effect between timing preference and information about booking policy regarding capacity, the parameter values of the profile combination ( $T 2 C 2$ ) were significant at the $95 \%$ confidence level. It indicated the profile that 7:30 pm with booking policy "at full capacity" and 10:00 pm with booking policy "more than capacity" have a positive influence on utility, while 7:30 pm with booking policy "more than capacity" and 10:00 pm with booking policy "at full capacity" have a negative effect. Two ways of thinking can be invoked. For the most preferred dinner time 7:30 pm, although booking policy "at full capacity" implies possible waiting, it is not as bad as "more than capacity" linked with certainty of waiting. But for the least preferred 
Table 5-3 Strategic dinner timing choice estimates (1-B)

\begin{tabular}{|c|c|c|c|c|}
\hline \multirow[b]{2}{*}{ Attribute } & \multicolumn{2}{|c|}{ RCL model } & \multicolumn{2}{|c|}{ MNL model } \\
\hline & Parameter & t-value & Parameter & t-value \\
\hline Intercept & $2.11^{*}$ & 3.94 & $2.25^{*}$ & 3.98 \\
\hline Time - 5:00 pm & 0.36 & 1.30 & 0.39 & 1.50 \\
\hline s.d. time $-5: 00 \mathrm{pm}$ & 1.00 & 1.76 & 0.69 & 1.45 \\
\hline Time $-7: 30 \mathrm{pm}$ & $2.14^{*}$ & 4.01 & $2.05^{*}$ & 4.36 \\
\hline s.d. time $-7: 30 \mathrm{pm}$ & $1.32^{*}$ & 2.66 & $1.21^{*}$ & 2.78 \\
\hline Booking close to capacity & $0.93^{*}$ & 5.18 & $0.95^{*}$ & 4.26 \\
\hline Booking at full capacity & 0.14 & 1.00 & -0.21 & -0.78 \\
\hline $\mathrm{T} 1 \mathrm{C} 1$ & na & & -0.19 & -0.70 \\
\hline $\mathrm{T} 1 \mathrm{C} 2$ & na & & 0.02 & 0.07 \\
\hline $\mathrm{T} 2 \mathrm{C} 1$ & na & & -0.07 & -0.23 \\
\hline $\mathrm{T} 2 \mathrm{C} 2$ & na & & $0.60^{*}$ & 2.15 \\
\hline Log likelihood null model & \multicolumn{2}{|c|}{-253.69} & \multicolumn{2}{|c|}{-253.69} \\
\hline Log likelihood final model & \multicolumn{2}{|c|}{-143.75} & \multicolumn{2}{|c|}{-140.80} \\
\hline Adjusted Rho-square & \multicolumn{2}{|c|}{0.43} & \multicolumn{2}{|c|}{0.43} \\
\hline
\end{tabular}

* significant at $\mathrm{p}<.05$ level; na $=$ not applicable.

time 10:00 pm, if the booking policy is "at full capacity", which is already brings with negative impact. It is difficult to explain the positive effect for the interaction of 10:00 pm with booking policy "more than capacity". After a close look at the data, we found two cases in which two respondents choose that alternative over others, which might imply particular personal preference due to heterogeneity. Overall, the results of this study could imply that for certain times that are considered popular times for an activity, such as 7:30 pm for dinner time, individuals seriously deal with the booking policy information about capacity that provides an indication of congestion.

Comparing Table 5-2 with Table 5-3 in terms of "dinner timing choice" estimates under the two scenarios, we observe that with the booking policy about capacity subjects behave more restrictively, as indirectly indicated by the variance of their timing preference. Therefore, we carried out the estimation across both scenarios to explore whether subjects' timing preferences differ significantly depending on whether or not 
they need to take into account the timing choices of others (i.e., the effect of strategic considerations). To test for such differences in timing preferences across the two scenarios in the "dinner timing choice" context, we estimated an additional random coefficients logit model jointly across the two dinner versions. In our analyses, we also tested for heteroscedasticity between the two versions, but found no significant differences in the error terms. We therefore report the results of the model without heteroscedasticity.

In this model, we allowed for differences in the size of the random coefficients between the two versions and also included the interaction of version with timing preference to test for differences in timing preference between the strategic and nonstrategic timing choices. When this interaction is significant, we conclude that timing preferences are significantly affected by the strategic nature of the task. The model estimated to perform this test is expressed as:

$$
\begin{aligned}
& U_{i t}^{d i n}=\alpha_{i t s t r a t}^{d i n}+\boldsymbol{\beta}_{\mathbf{p o l}}^{\mathrm{din}} \mathbf{X}_{\mathbf{p o l}}+\boldsymbol{\beta}_{\text {wait }}^{\mathrm{din}} \mathbf{X}_{\mathbf{w a i t}}+\boldsymbol{\beta}_{\text {add }}^{\mathrm{din}} \mathbf{X}_{\text {addwait }}+\varepsilon_{i t k}^{d i n} \\
& \alpha_{i t s t r a t}^{d i n}=\alpha^{d i n}+\beta_{\text {strat }}^{d i n} X_{\text {strat }}+\left(\gamma_{\mathbf{t}}^{\mathrm{din}}+\gamma_{\text {strat }}^{\mathrm{din}} X_{\text {strat }}+\boldsymbol{v}_{\mathrm{itstrat}}^{\mathrm{din}}\right) \mathbf{X}_{\mathbf{t}}
\end{aligned}
$$

where $\beta_{\text {strat }}^{\text {din }} X_{\text {strat }}$ is a main effect of task version (strategic vs. non-strategic) on the utility for a time slot, $\gamma_{\text {strat }}^{\text {din }} X_{\text {strat }} \mathbf{X}_{\mathrm{t}}$ is the interaction effect of task version and time slot preference, $\boldsymbol{v}_{\mathrm{itstrat}}^{\text {din }}$ is a normally distributed individual, time and task specific random component; and all other components are defined as before.

Results for the "dinner timing choice" section of the experiment are reported in Table 5-4. The first column presents the parameter estimates for the version with strategic consideration. A total of 61 individuals participated in this version. The overall fit of the model is good, with an adjusted pseudo Rho-squared value of 0.43 . One of the timing preference parameters and one of the capacity levels were significant at the $95 \%$ confidence level. Subjects showed a clear preference for a dinner time at 7:30 pm. They did not like to have dinner at 5:00 pm or 10:00 pm and there was no significant difference in preference between these two alternative times. We also see that the information about booking at "close to capacity", "at full capacity", or "more than capacity" plays a role in the decision-making process, with booking at "close to capacity" having a positive effect over the other restaurant booking policy as expected. This finding supports the notion of subjects' strategic anticipation of congestion because it indicates that they are concerned about whether or not they will have to wait in case congestion occurs.

The second column in Table 5-4 presents the parameter estimates for the version without strategic consideration. A total of 57 individuals participated in this version. The overall fit of the model is also good, with an adjusted pseudo Rho-squared value of 0.48 . In this model, all parameter values were significant at the $95 \%$ confidence level. As expected, increased waiting time and a greater probability of additional wait both negatively impacted utility.

The results of the comparison of the models (equation 5.4) showed that the timing preference parameters of two models are significantly different between the versions. This is evidenced by a significant interaction of version with timing preference. The 
Table 5-4 Dinner timing choice model estimates (1-A \& 1-B $)^{\S}$

\begin{tabular}{|c|c|c|c|c|}
\hline \multirow[b]{2}{*}{ Attribute } & \multicolumn{2}{|c|}{ Strategic } & \multicolumn{2}{|c|}{ Non-strategic } \\
\hline & Parameter & t-value & Parameter & t-value \\
\hline Intercept & $2.11^{*, a}$ & 3.94 & $5.36^{*, a}$ & 10.98 \\
\hline Time $-5: 00 \mathrm{pm}$ & $0.36^{\mathrm{a}}$ & 1.30 & $-1.03^{*, \mathrm{a}}$ & -3.10 \\
\hline s.d. time $-5: 00 \mathrm{pm}$ & 1.00 & 1.76 & $1.93^{*}$ & 5.73 \\
\hline Time $-7: 30 \mathrm{pm}$ & $2.14^{*, a}$ & 4.01 & $3.54^{*, a}$ & 8.17 \\
\hline s.d. time $-7: 30 \mathrm{pm}$ & $1.32^{*}$ & 2.66 & $2.05^{*}$ & 5.63 \\
\hline Waiting time & na & & $-0.16^{*}$ & -10.71 \\
\hline Probability of additional wait & na & & $-0.04^{*}$ & -7.41 \\
\hline Booking close to capacity & $0.93^{*}$ & 5.18 & na & \\
\hline Booking at full capacity & 0.14 & 1.00 & na & \\
\hline Log likelihood null model & \multicolumn{2}{|c|}{-253.69} & \multicolumn{2}{|c|}{-711.17} \\
\hline Log likelihood & \multicolumn{2}{|c|}{-143.75} & \multicolumn{2}{|c|}{-365.34} \\
\hline Adjusted Rho-square & \multicolumn{2}{|c|}{0.43} & \multicolumn{2}{|c|}{0.48} \\
\hline
\end{tabular}

$\S$ number of observations "strategic" is 183, for 61 individuals; number of observation for "nonstrategic" 513, for 57 individuals; na = not applicable.

* significant at $\mathrm{p}<.05$ level.

${ }^{\mathrm{a}}$ non strategic estimate is significantly different from strategic choice task estimate at $\mathrm{p}<.05$ level.

results of the analysis showed that the model fit was acceptable with an adjusted pseudo Rho-squared value of 0.41 and provided us with a significant estimate of time interaction of -0.58 and 0.71 for time 5:00 pm and 7:30 pm respectively (with $t$-value of -3.21 and 2.7 correspondingly).

In line with what one would expect if strategic anticipation of other individuals' choices occurs, we find that subjects' timeg preferences were significantly lower for the most popular time of 7:30 pm in the strategic choice scenarios. Interestingly, subjects were also more inclined to dine earlier $(5: 00 \mathrm{pm})$ in the strategic scenarios than in the non-strategic scenarios, possibly motivated by the fact that they anticipated this to be the least congested time slot. 


\subsubsection{Participation timing choice model}

As input for the equilibrium simulations and as a baseline for non-strategic timing choice model, we estimated a random coefficients model of subjects' participation timing choices that expresses subjects' non-strategic timing preferences of participating in the experiment. The model is expressed as:

$$
\begin{aligned}
& U_{i t}^{\text {part }}=\alpha_{i t}^{\text {part }}+\boldsymbol{\beta}_{\text {wait }}^{\text {part }} \mathbf{X}_{\text {wait }}+\boldsymbol{\beta}_{\text {add }}^{\text {part }} \mathbf{X}_{\text {addwait }}+\varepsilon_{i t k}^{\text {part }} \\
& \alpha_{i t}^{\text {part }}=\alpha^{\text {part }}+\left(\gamma_{\mathbf{t}}^{\text {part }}+\mathbf{v}_{\mathbf{i t}}^{\text {part }}\right) \mathbf{X}_{\mathbf{t}}
\end{aligned}
$$

where all components are defined as before, but now refer to the participation timing choice.

The model estimated on strategic participation timing choice data (1-C) provided us with a parameter estimate $\left(\beta_{\text {con }}^{\text {partcon }}\right)$ for the effect of variations in the anticipated number of other subjects $\left(X_{c o n}\right)$ at a given time slot $(t)$ on subjects utility for this time slot $\left(U_{i t}^{\text {partcon }}\right)$. The model used to perform this estimation is expressed as:

$$
\begin{aligned}
& U_{i t}^{\text {partCon }}=\alpha_{i t}^{\text {partCon }}+\beta_{\text {con }}^{\text {partCon }} X_{c o n}+\varepsilon_{i t k}^{\text {partCon }} \\
& \alpha_{i t}{ }^{\text {artCon }}=\alpha^{\text {partCon }}+\left(\boldsymbol{\gamma}_{\mathbf{t}}^{\text {partCon }}+\mathbf{v}_{\mathbf{i t}}^{\text {partCon }}\right) \mathbf{X}_{\mathbf{t}}
\end{aligned}
$$

The results of our model estimated for the two scenarios of "participation timing choices" are presented in Table 5-5. The test statistics show that in both scenarios both the RCL and the MNL models outperform the model with all parameters equal to zero (null model). It appears that the parameters for timing preference are somehow consistent through the two scenarios: respondents show a clear preference for attending the experiment at 2:30 pm. They do not like to participate at 4:00 pm, and really dislike attending at 5:30 pm. In the strategic scenario, the information about attendance brings about a negative effect, as in the non-strategic scenario, for waiting time and probability of additional delay, which is in line with our expectations. Comparing the estimation results between the two scenarios, the results suggest that providing congestion information moderates the range difference in the timing preferences. We also observed that subjects behave more restrictively if they have congestion information, as indirectly indicated by the variance of their timing preferences.

For the strategic situation, likely because of insufficient observations, some of the RCL model results are not significant at the 95\% confidence level. It is hard to use the similar method as expressed in equation 5.4. In order to further explore the effect of providing congestion information (e.g., whether the timing preferences differ significantly in two scenarios), we conducted a Chi-square test using the frequency of the time choices. Due to the full-factorial design, the frequencies of the choices reflect the pure timing preferences for both scenarios (Table 5-6). The result is significant at the 95\% confidence level with a value of 13.44 at three degrees of freedom. This finding is also supportive of 
Table 5-5 Participation timing choice estimates $(2-B \& 2-C)^{\S}$

\begin{tabular}{|c|c|c|c|c|}
\hline \multirow[b]{2}{*}{ Attribute } & \multicolumn{2}{|c|}{ Strategic } & \multicolumn{2}{|c|}{ Non strategic } \\
\hline & Parameter & t-value & Parameter & t-value \\
\hline Intercept & $2.09^{*}$ & 2.74 & $5.36^{*}$ & 17.16 \\
\hline Time $-2: 30 \mathrm{pm}$ & $2.74^{*}$ & 4.24 & $4.32^{*}$ & 11.85 \\
\hline s.d. time $-2: 30 \mathrm{pm}$ & 2.22 & 1.6 & $2.46^{*}$ & 7.86 \\
\hline Time $-4: 00 \mathrm{pm}$ & $1.49^{*}$ & 3.29 & $1.30^{*}$ & 3.58 \\
\hline s.d. time $-4: 00 \mathrm{pm}$ & 0.17 & 0.13 & $2.51^{*}$ & 7.92 \\
\hline Waiting time & na & & $-0.31^{*}$ & -17.52 \\
\hline Probability of additional wait & na & & $-0.13^{*}$ & -15.26 \\
\hline Expected registration number & $-0.08^{*, a}$ & -3.31 & na & \\
\hline Log likelihood null model & \multicolumn{2}{|c|}{-163.58} & \multicolumn{2}{|c|}{-1472.25} \\
\hline Log likelihood & \multicolumn{2}{|c|}{-109.37} & \multicolumn{2}{|c|}{-571.08} \\
\hline Adjusted Rho-square & \multicolumn{2}{|c|}{0.32} & \multicolumn{2}{|c|}{0.61} \\
\hline
\end{tabular}

$\S$ Number of observations "non-strategic" is 1062, for 118 individuals; number of observations "strategic" is 118 , for 118 individuals; na = not applicable.

* significant at $\mathrm{p}<.05$ level.

${ }^{\mathrm{a}}$ MNL model estimate is -0.058 at $\mathrm{p}<.05$ level.

the notion that subjects' timing preferences differ significantly depending on whether or not they are provided with congestion information. The results seem to indicate that individuals took the congestion information to be truthful and that after correcting their preferences for congestion levels, they were more inclined to choose their originally most preferred time.

Table 5-6 Observed participation timing choice frequencies

\begin{tabular}{|l|c|c|c|c|}
\hline Scenario & Time $-2: 30 \mathrm{pm}$ & Time $-4: 00 \mathrm{pm}$ & Time $-5: 30 \mathrm{pm}$ & None \\
\hline Non-strategic & 62 & 27 & 23 & 6 \\
\hline Strategic & 67 & 32 & 9 & 10 \\
\hline
\end{tabular}




\subsubsection{Non-strategic and strategic behaviour}

After getting some insight into preference for "dinner timing choice" and "participation timing choice" separately, we go one step further to explore whether this type of preference is similar across the two activity contexts both in the non-strategic situation and the strategic situation.

Table 5-7 presents the parameter estimates using the random coefficients logit model for the situations without strategic consideration, using data sets of both "dinner timing choice" (1-A) and "participation timing choice" (2-B). The number of choice sets and number of observations is 513 and 2052 in (1-A), and 1062 and 4248 in (2-B). The number of parameters to be estimated is seven in both cases. The test statistics show that the models outperform the model with all parameter equal to zero (null model). Moreover, it is confirmed that waiting time and uncertainty of additional wait bring on a negative effect for both activities. After correcting for the effect of waiting time and uncertainty, subjects show some common trend for timing preferences to undertake the activity, and they also show significant individual heterogeneity in timing preference. Comparing the estimation results between the two activities, the results suggest that the timing preference is more extreme for "dinner timing choice" than "participation timing choice", which can be explained by the nature of the activity. That is, dinner timing gives more common constraints for fixed time cross the population.

Table 5-7 Non-strategic behaviour estimates (1-A \& 2-B)

\begin{tabular}{|l|c|c|c|c|}
\hline & \multicolumn{2}{|c|}{ Dinner timing choice } & \multicolumn{2}{c|}{ Participation timing choice } \\
\hline Attribute & Parameter & t-value & Parameter & t-value \\
\hline Intercept & $5.36^{*}$ & 10.98 & $5.36^{*}$ & 17.16 \\
\hline Time 1 & $-1.03^{*}$ & -3.10 & $4.32^{*}$ & 11.85 \\
\hline s.d. time 1 & $1.93^{*}$ & 5.73 & $2.46^{*}$ & 7.86 \\
\hline Time 2 & $3.54^{*}$ & 8.17 & $1.30^{*}$ & 3.58 \\
\hline s.d. time 2 & $2.05^{*}$ & 5.63 & $2.51^{*}$ & 7.92 \\
\hline Waiting time & $-0.16^{*}$ & -10.71 & $-0.31^{*}$ & -17.52 \\
\hline Probability of additional wait & $-0.04^{*}$ & -7.41 & $-0.13^{*}$ & -15.26 \\
\hline Log likelihood null model & & & & \\
\hline Log likelihood final model & -711.17 & & -1472.25 \\
\hline Adjusted Rho-square & & & & -571.08 \\
\hline
\end{tabular}

* significant at $\mathrm{p}<.05$ level; na = not applicable. 
In the non-strategic situation, to test whether the participants' timing choice estimates differ significantly depending on the activity type, the following analyses were performed across the two timing choice data sets. For each pair of data sets an additional variable was created to measure the contrast between the parameters of two data sets. In the first data set, these contrast variables multiplied by " +1 ", which were equal to the original variables; whereas for the second data set, these contrast variables multiplied by "-1", which were defined as the negative values of the original ones. If the corresponding contrast parameter appears to be significantly different from zero, it can be concluded that the parameter is different for two data sets. In addition, we checked the scale difference of the two data sets to make sure that the difference is not caused by a scale factor.

In Table 5-8, the number of choice sets and number of observations is 513 and 2052 in the "dinner timing choice" (1-A), and 1062 and 4248 in the "participation timing choice" (2-B). The number of parameters to be estimated is nine in both cases. The test statistics show that the models outperform the model with all parameter equal to zero (null mode). The model fit was good with an adjusted pseudo Rho-squared value of 0.51 .

As Table 5-8 shows, the parameter estimates for waiting time and uncertainty are significantly different for the two data sets. That is, for different activities, subjects use different weights for the same variables such as waiting time and probability about additional delay to access the utility when making their timing decision.

Table 5-8 Non-strategic behaviour contrast parameter estimates (1-A \& 2-B)

\begin{tabular}{|c|c|c|c|c|c|}
\hline & & \multicolumn{2}{|c|}{ Data operation } & \multicolumn{2}{|c|}{ Estimated result } \\
\hline & Data set & $\begin{array}{l}\text { Participation } \\
\text { timing choice }\end{array}$ & $\begin{array}{l}\text { Dinner timing } \\
\text { choice }\end{array}$ & Parameter & t-value \\
\hline & Intercept & & & $5.12^{*}$ & 25.66 \\
\hline \multirow{4}{*}{$\begin{array}{l}\text { Original } \\
\text { variables }\end{array}$} & Time 1 & & & $0.46^{*}$ & 6.31 \\
\hline & Time 2 & & & $1.09^{*}$ & 10.82 \\
\hline & Waiting time & & & $-0.18^{*}$ & -22.60 \\
\hline & Probability of additional wait & & & $-0.07^{*}$ & -17.74 \\
\hline \multirow{4}{*}{$\begin{array}{l}\text { Contrast } \\
\text { variables }\end{array}$} & Time 1 Contrast & \multirow{4}{*}{$\times(+1)$} & \multirow{4}{*}{$\times(-1)$} & $1.07^{*}$ & 13.74 \\
\hline & Time 2 Contrast & & & $-1.45^{*}$ & -14.74 \\
\hline & Waiting time Contrast & & & $-0.04^{*}$ & -6.74 \\
\hline & $\begin{array}{l}\text { Probability of additional wait } \\
\text { Contrast }\end{array}$ & & & $0.02^{*}$ & -7.72 \\
\hline
\end{tabular}

* significant at $\mathrm{p}<.05$ level. 
Table 5-9 presents the parameter estimates using the random coefficients logit model for the situations with strategic consideration, using data sets of both "dinner timing choice" (1-B) and "participation timing choice" (2-C). The number of choice sets and number of observations is 183 and 732 in (1-B), and 118 and 472 in (2-C). The number of parameters to be estimated is seven and six respectively. The test statistics show that the models outperform the model with all parameter equal to zero (null model). After correcting for the effect of information of booking policy and information of expected registration, subjects show some common trend for timing preferences to undertake the activity, and they also show some significant individual heterogeneity in timing preferences. Comparing the estimation results between the two activities, the results suggest that timing preferences are more extreme for dinner timing choice than participation timing choice, which are consistent with the results of without strategic consideration and correlated by the nature of the activity. That is, a fixed time for dinner is common sense for most people. Moreover, in the strategic situation, the estimation results show that timing preferences are moderate compared with the non-strategic situation, both for "dinner timing choice" and "participation timing choice".

We conducted similar contrast parameter estimations with strategic behaviour for "participation timing choice" data set (2-C) and "dinner timing choice" data set (1-B). In order to make a comparison, we recoded the registration information of the experimental

Table 5-9 Strategic behaviour estimates (1-B \& 2-C)

\begin{tabular}{|l|c|c|c|c|}
\hline & \multicolumn{2}{|c|}{ Dinner timing choice } & \multicolumn{2}{c|}{ Participation timing choice } \\
\hline Attribute & Parameter & t-value & Parameter & t-value \\
\hline Intercept & $2.11^{*}$ & 3.94 & $2.09^{*}$ & 2.74 \\
\hline Time 1 & 0.36 & 1.30 & $2.74^{*}$ & 4.24 \\
\hline s.d. time 1 & 1.00 & 1.76 & 2.22 & 1.6 \\
\hline Time 2 & $2.14^{*}$ & 4.01 & $1.49^{*}$ & 3.29 \\
\hline s.d. time 2 & $1.32^{*}$ & 2.66 & 0.17 & 0.13 \\
\hline Close to capacity & $0.93^{*}$ & & na & \\
\hline At full capacity & 0.14 & & na & -3.31 \\
\hline Expected registration number & na & & $-0.08^{*}$ & -163.58 \\
\hline Log likelihood null model: LL(0) & & -253.69 & & -109.37 \\
\hline Log likelihood final model: LL(B) & \multicolumn{2}{|c|}{-143.75} & & \\
\hline Adjusted Rho-square & \multicolumn{2}{|c|}{0.43} & & \\
\hline
\end{tabular}

${ }^{*}$ significant at $\mathrm{p}<.05$ level; na $=$ not applicable. 
timing by setting the expected waiting time that would occur if the number of participants at any given time slot would be greater than 25 . That is, if the information says the number of subjects that would register is 17 , then we coded it as "close to capacity", if it says the number is 33 then coded it as "at full capacity" and if the number of was 50 coded it as "more than capacity". The results are shown in Table 5-10.

In Table 5-10, the number of choice sets and number of observations is 183 and 732 in the "dinner timing choice" (1-B), and 118 and 472 in the "participation timing choice" (1-C). The number of parameters to be estimated is five in both cases. The test statistics show that the models outperform the model with all parameter equal to zero (null model). The model fit is acceptable with an adjusted pseudo Rho-squared value of 0.38 . The results of the estimated contrast parameters imply that the impact of information about capacity is not significantly different across the two activities. This offers some support for the notion that individuals dislike congestion in general, which might be independent from activity type.

After investigating preference for different activities, we go one step further to obtain insights into individuals' reactions to different information of participation distribution. The data set of the "participation timing choice" with strategic consideration (2-C) were further analysed to explore how subjects' timing preferences may be influenced by the specific information about the participation distribution of others. As

Table 5-10 Strategic behaviour contrast parameter estimates (1-B \& 2-C)

\begin{tabular}{|c|c|c|c|c|c|}
\hline & & \multicolumn{2}{|c|}{ Data operation } & \multicolumn{2}{|c|}{ Estimated result } \\
\hline & Data set & $\begin{array}{l}\text { Participation } \\
\text { timing choice }\end{array}$ & $\begin{array}{l}\text { Dinner timing } \\
\text { choice }\end{array}$ & Parameter & t-value \\
\hline & Intercept & & & $1.47^{*}$ & 5.17 \\
\hline \multirow{4}{*}{$\begin{array}{l}\text { Original } \\
\text { variables }\end{array}$} & Time 1 & & & $0.58^{*}$ & 5.29 \\
\hline & Time 2 & & & $0.77^{*}$ & 5.56 \\
\hline & Information close to capacity & & & $0.82^{*}$ & 7.20 \\
\hline & Information at full capacity & & & 0.13 & 1.06 \\
\hline \multirow{4}{*}{$\begin{array}{l}\text { Contrast } \\
\text { variables }\end{array}$} & Time 1 Contrast & \multirow{4}{*}{$\times(+1)$} & \multirow{4}{*}{$\times(-1)$} & $0.43^{*}$ & 3.94 \\
\hline & Time 2 Contrast & & & $-0.66^{*}$ & -4.74 \\
\hline & $\begin{array}{l}\text { Information close to capacity } \\
\text { Contrast }\end{array}$ & & & 0.05 & 0.46 \\
\hline & $\begin{array}{l}\text { Information at full capacity } \\
\text { Contrast }\end{array}$ & & & -0.06 & -0.51 \\
\hline
\end{tabular}

* significant at $\mathrm{p}<.05$ level. 
Table 5-11 Information impacts (2-C)

\begin{tabular}{|l|c|c|c|c|c|c|}
\hline & \multicolumn{2}{|c|}{$\begin{array}{r}\text { Information about number of students } \\
\text { would prefer for each time }\end{array}$} & \multicolumn{3}{c|}{ Number of students } \\
& Time 1 & Time 2 & Time 3 & Time 1 & Time 2 & Time 3 \\
\hline Information set 1 & 17 & 33 & 50 & 93 & 7 & 0 \\
\hline Information set 2 & 17 & 50 & 33 & 73 & 13 & 13 \\
\hline Information set 3 & 33 & 17 & 50 & 28 & 72 & 0 \\
\hline Information set 4 & 33 & 33 & 33 & 81 & 13 & 6 \\
\hline Information set 5 & 33 & 50 & 17 & 100 & 0 & 0 \\
\hline Information set 6 & 50 & 17 & 33 & 33 & 60 & 7 \\
\hline Information set 7 & 50 & 33 & 17 & 28 & 36 & 36 \\
\hline
\end{tabular}

described above, for the experiment participation time, subjects prefer 2:30 pm most, then 4:00 pm, and they dislike 5:30 pm. But when we look at the percentage of students choosing each time after offering different information about the distribution of attendance, we get a very interesting picture. The percentage of subjects choosing each time slot under seven information sets is shown in Table 5-11. The number of subjects choosing each time is scaled to a sum of 100 across three time slots.

Under information set 4 (that represents a uniform distribution), the choice frequency reflects more about the timing preference and less about the impact of interaction. This scenario depicted more or less the same scenario with information implying no congestion for a particular time, explaining the prevalence of timing preference. Under information set 7 (that reflects more or less the actual distribution), the choice frequency shows a contrary preference distribution, which is a rather balanced distribution. This demonstrates partly the case that individuals have a very strong dislike of congestion and anticipate that other individuals may not dislike congestion, so that fewer individuals end up using a service at their most preferred time. Under information set 1 (that indicates the opposite of the actual distribution), the choice frequency is pushed to a more extreme distribution, enforcing the original preference. This reflects the possible situation that all individuals may choose to go at the time that they originally prefer most, if none of them anticipates the occurrence of congestion. In general, we could observe that if the information reflects the actual preference that is consistent with common intuition, it leads to some kind of balance, whereas if the information is the opposite of the actual preference, it leads to an enforcement of original preferences. This proves that individuals can trade-off their preference and anticipation of congestion, expecting that others make the same trade-off and that they rely to some degree on the provided information. Thus, by manipulating information within some range, individuals' behaviour can be shifted into a certain direction in terms of timing choice. 
In summary, the data analyses supported our primitive assumptions, which was the foundation that we used to construct our strategic behavioural models in the first place. Next, we investigated our hypothesis using actual participation timing choice as a case study.

\subsubsection{Test of hypothesis}

The choice of participation time for attending the experiment is not a routine activity in daily life. Participants in general have less opportunity to learn what to expect about others' choice, especially in our settings no information about others' timing choices is provided. Because of this, the experiment could be considered as a one-shot game in which participants use noisy introspection to anticipate others' choice.

On the other hand, participants come from the same sub-group of the population with similar backgrounds and similar daily schedules. They are university undergraduate students. This may lead to more or less similar ways of thinking and reasoning, which might fit the quantal response framework, or even the Nash equilibrium. Comparing the performance of the proposed model that is used to predict the expectation of subjects in terms of three conjecture ability levels with their actual choices, the most appropriate model that best predicts participation time choice can be identified. Thus, it could provide an answer to our hypothesis.

Hypothesis: In one-shot strategic situations, a quantal response model leads to more precise predictions of strategic (participation timing) choice behaviour compared to a Nash equilibrium model, but a model of noisy introspection predicts even better.

Using the estimates of the "participation timing choice" model as a starting point, we simulated the (equilibrium) outcomes that also take into account whether or not subjects strategically anticipate other subjects' choices. To do this, we simulated the participation timing choices for ten times the total number of observed subjects $(n=118)$, based on the estimated timing preference vector and the distribution of timing preferences within the sample (see equation 5.4 and Table 5-5).

To incorporate the anticipated effect of congestion, we included in the non-strategic choice model a proxy for subjects' disutility of congestion (using data set (2-C) and equation 5.7, which is modified from equation 5.5), as well as each subject's anticipated number of other participants at each time slot.

$U_{i t}^{\text {partCon }}=\alpha_{t}^{\text {partCon }}+\beta_{c o n}^{\text {partCon }} X_{c o n}+\varepsilon_{i t k}^{\text {partCon }}$

The results of the analysis show that the model fit is acceptable with an adjusted pseudo Rho-squared of 0.32 and provided us with a significant estimate of congestion disutility of -0.058 per additional individual (with a $t$-value of -5.21). The average number of the anticipated other subjects for each time slot was 43.9 for the $2: 30 \mathrm{pm}$ time slot, 28.5 for the 4:00 pm time slot and 15.9 for the 5:30 pm time slot. 
In particular, the systematic expected payoff function that was used in the simulation is:

$E\left[\bar{U}_{t}\right]=\alpha_{t}+\theta\left((N-1) p_{t}^{*}+1\right)$

The following procedure was adopted in the simulations:

1. Draw $10 \times 118$ sets of random values for the preference vector, from a normal density distribution with the observed means (estimates of 4.32, 1.30 for time slots 2:30 $\mathrm{pm}$ and 4:00 pm respectively) and the variances for the time slots (2.46 and 2.51). Every set defines an individual's type as derived from the estimation results.

2. Use these values to calculate the probabilities that each individual will choose a certain time slot when no strategic anticipation occurs.

3. Use these values along with subject's average self-reported anticipated number of other participants at each time slot along with a proxy for their disutility of congestion (-0.058 per individual) to calculate the probabilities that each individual chooses a certain time slot when strategic anticipation does occur. In this probability calculation, we include different values of $\mu$ depending on the equilibrium type (see equation 3.8). Since it is not possible for $\mu$ to take on a value of exactly zero in the simulation (as would be required for the Nash equilibrium), a value close to zero was used to approximate the resulting distribution of demand ( $\mu=0.5)$, meaning that individuals play the best strategy with a probability that is very close to 1 . Following observations made by McKelvey and Palfrey (1995), the values $\mu_{0}=6.6$ and $t=1.0$ were used to simulate the quantal response equilibrium. This latter equilibrium has the property that each individual's probabilities are a logit stochastic response to the others. The values $\mu_{0}=4.4$ and $t=4.1$, adopted from Goeree and Holt (2004) were used to simulate the noisy introspection model, which assumes the lowest rationality.

4. Sum the simulated probabilities for each start time across individuals, generating a distribution of demand of 118 individuals, the number that actually participated.

Figure 5-2 illustrates the different distributions of demand that arise based on the Nash equilibrium, the quantal response equilibrium, the noisy introspection model and the choice model without strategic anticipation. To investigate subjects' ability to anticipate correctly how other subjects would distribute over the different time slots, Table 5-12 reports the subjects' average expected number of participants for each time slot (scaled from their self-reported total of 88 to the total of 118 used in the simulation). Finally, the actual distribution of demand is presented as benchmark for the simulations and subjects' self-reported expectations.

Based on a Chi-square analysis of the predicted distribution of demand relative to the actual distribution of demand, we find that the non-strategic time choice model is the closest to the actual timing choices. Of the strategic models, both the quantal equilibrium model and the noisy introspection model differ significantly in terms of predicted 


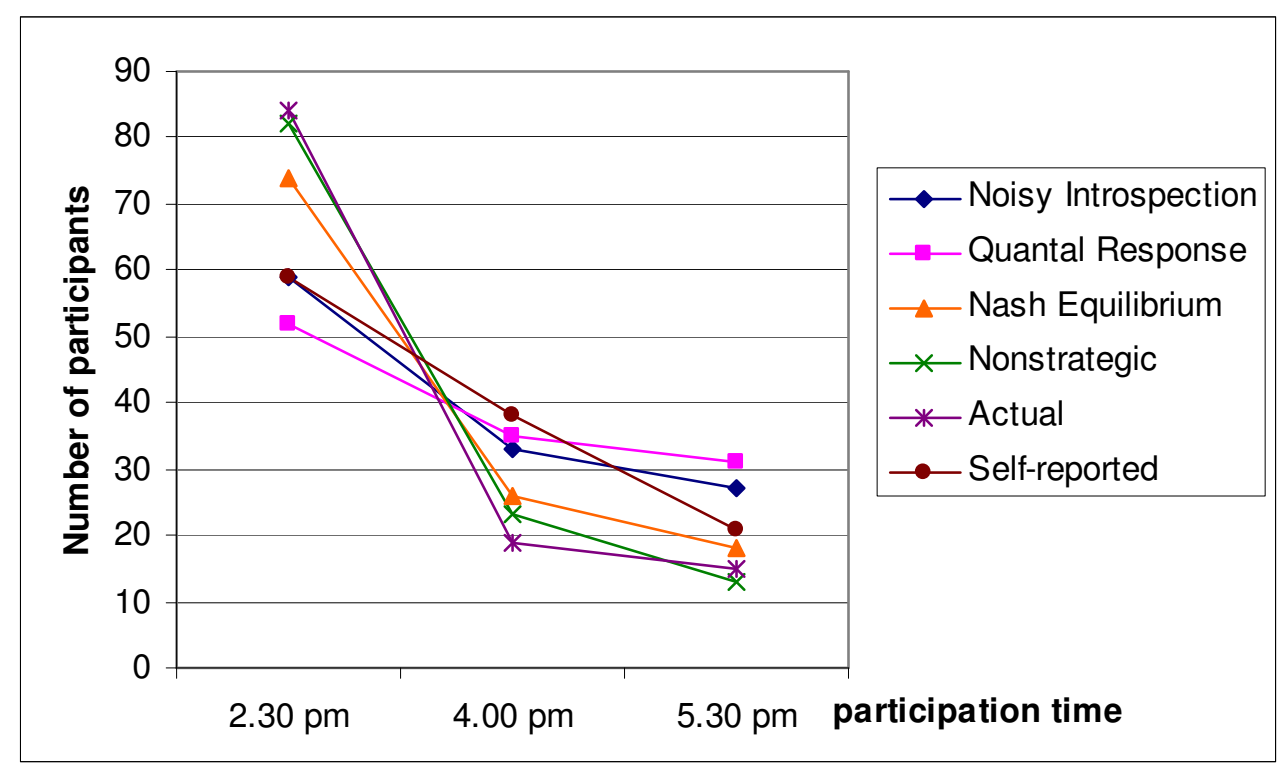

Figure 5-2 Simulations of participation timing choice

numbers from the actual observed numbers of choices in each time slot $(p<.05)$. Of the three models, the Nash equilibrium predicts a distribution that is considerably closer to the observed attendance levels, and not significantly different.

Interestingly, subjects' self-reported expected number of other participants in each time slot is placed in between the projected quantal response equilibrium and the Nash

Table 5-12 Simulations of participation timing choice

\begin{tabular}{|c|c|c|c|}
\hline Distribution of demand $(n=118)$ & $2: 30 \mathrm{pm}$ & $4: 00 \mathrm{pm}$ & $5: 30 \mathrm{pm}$ \\
\hline Noisy introspection ${ }^{a}$ & $59(50 \%)$ & $33(28 \%)$ & $26(22 \%)$ \\
\hline Quantal response $^{\mathrm{a}}$ & $52(44 \%)$ & $35(30 \%)$ & $31(26 \%)$ \\
\hline Nash equilibrium ${ }^{b}$ & $74(63 \%)$ & $26(22 \%)$ & $18(15 \%)$ \\
\hline $\begin{array}{l}\text { Non-strategic time choice model without } \\
\text { anticipated congestion }{ }^{b}\end{array}$ & $82(70 \%)$ & $23(19 \%)$ & $13(11 \%)$ \\
\hline Actual attendance ${ }^{b}$ & $84(71 \%)$ & $19(16 \%)$ & $15(13 \%)$ \\
\hline $\begin{array}{l}\text { Subjects self-reported expected number } \\
\text { of other participants }\end{array}$ & $59(50 \%)$ & $38(32 \%)$ & $21(18 \%)$ \\
\hline
\end{tabular}

${ }^{a}$ significantly different $(\mathrm{p}<.05)$ from actual attendance in a Chi-square test.

${ }^{\mathrm{b}}$ significantly different $(\mathrm{p}<.05)$ from self-reported expected number of other participants.

${ }^{£}$ scaled up from the self-reported total of 88 to the total of 118 used in the simulation. 
equilibrium and does not differ significantly from the noisy introspection projections. In contrast, the self-reported expectations differ significantly from the actual distribution of attendance and from the non-strategic model projections $(p<.05)$.

The analysis of the participation time data provided mixed support for the fact that individuals anticipate other individuals' timing choices. On the one hand, participants reported an expected distribution of demand that was in line with the noisy introspection projection. Thus, the results provide support for the notion that individuals are capable of anticipating other individuals' timing choices and base their anticipation on a strategic behaviour model. On the other hand, the results also showed that when choosing their actual participation times, participants did not take into account the potential waiting they had to incur due to congestion caused by the presence of the anticipated other participants. This latter conclusion is evidenced by the fact that the model projections based on a (non-strategic) model without anticipated waiting did not differ significantly from the actual choices subjects had made.

There are a number of potential explanations for the mixed results in the participation time choice data that we now explore in greater detail. First, one might suspect that although participants anticipated other participants to come at a given time, participants may not have anticipated an actual waiting time. Our observations on subjects' self-reported expected waiting time, however, show that this was not the case. On average, participants expected to wait 10.9 minutes for the $2: 30 \mathrm{pm}$ time slot, 7.3 minutes for the 4:00 pm time slot and 4.6 minutes for the 5:30 pm time slot and the nonstrategic results reported in Table 5-4 show that subjects did indeed have a significant negative utility for waiting in the context of this experiment. Thus, it appears that participants for some reason did not incorporate the anticipated disutility of congestion in their actual timing choices.

An explanation for this finding may be that subjects are relatively certain about their timing preferences whereas they are uncertain about the potential congestion at each time slot. This information structure may suggest that rather than correcting the entire trade-off for differences in the scale parameter $\mu$ (as is done in the quantal response equilibrium) perhaps only parameters for strategically influenced variables (such as congestion) should be corrected. Such an approach would lower the relative impact of congestion compared to timing preferences and would result in projections that are closer to the model without strategic anticipation.

An alternative explanation may be that in response to hypothetical timing choices, subjects tend to respond in a relatively general fashion without thinking of a specific date and therefore do not sufficiently take into account future personal time constraints such as time budget and scheduling restrictions that they may face on the actual experiment date. For example, in making their actual choices, subjects may have realized that they had fixed dinner times that they did not wish to change while in a hypothetical choice regarding some future date they may feel that they could easily avoid congestion and have a late dinner. Similar to findings in attitudinal research, it may be the case in our empirical application that positive evaluations of a certain time slot, may not translate into action due to, for example, social pressure (e.g., Ajzen, 1991). Future research could address the question of how to incorporate such individual time constraints more directly into models of individual timing choice. 


\subsection{Conclusion and discussion}

This study set out to address the question if, and if so how, individuals anticipate what time slots are most popular among other individuals when making their own activity timing choices in a one-shot situation. We now interpret our results to provide an answer to this question.

First, based on the differences in timing preferences between the strategic and nonstrategic dinner timing choice data, we concluded that individuals were able to anticipate other individuals' dinner timing choices, and did indeed adjust their own timing choices accordingly to avoid waiting due to congestion.

Second, based on the differences in timing preference across the "dinner timing choice" and the "participation timing choice" data in a non-strategic situation as well as in a strategic situation, we confirmed that individuals have similar preferences for activity participation times and dislike congestion, but have different distributions of originally preferred times for different activities.

Third, based on the shift in participation time choices after receiving the specific information about the distribution of others, we proved that individuals adjust their strategic anticipation of congestion through information or experience about congestion.

Fourth, the analyses of the participation timing choice data provided mixed support for the formulated hypothesis. It provides evidence of the fact that in this strategic situation individuals anticipate other individuals' timing choices. It showed that in this specific case individuals may not always take into account the congestion that they anticipate when making actual timing choices.

Having the positive result that people are able to anticipate congestion, the next question about the proposed model is whether learning taking place in a strategic context. To further test our hypothesis, we set out our next experiment to test the potential role of learning about congestion in repeated strategic situations (e.g., when learning about congestion in their favourite restaurant), and to analyse to what extent information provision may influence individuals' timing choices to achieve a more equal distribution of demand and reduce the potential burdens of congestion. 


\section{Chapter 6}

\section{Repeated Strategic Choice}

\subsection{Introduction}

In the previous chapter, we addressed the question whether, and if so, how individuals anticipated which time slots are most popular for other individuals when making their own activity timing decisions. The results of our experiment on timing choice decisions supported the notion that individuals are able to anticipate other individuals' timing choices, and adjust their own timing choices accordingly to avoid congestion.

In this chapter, we extend this research by investigating how individuals learn to incorporate strategic considerations with respect to congestion in repeated choice situations, and to what extent individuals' learning as well as strategic choices are influenced by providing recommendations.

In every day life, there are many choices that are repeated on a daily basis, such as choice of travel mode, departure time, and route choice in the context of commuter behaviour. Some other choices are happening very frequently, such as visiting a local pub or a local restaurant. In these repeated choice situations, individuals build up a substantial amount of experience with a population (or have heard about the general nature of the population from organizations or friends). Consequently, their strategic behaviour in anticipation of congestion may be somewhat different from that in situations that only happen once. They may be able to more precisely anticipate timing choice of others as they learn. Thus, the reasoning that is addressed in the equilibrium model will be more 
appropriate rather than that is addressed in the noisy introspection model. Moreover, they may act strategically when provided with recommendations (such as congestion information en-route, or crowdedness information of the restaurant). By taking into account how others react to recommendation, they may or may not choose to follow the recommendation, based on their modified anticipation of congestion.

Testing the proposed model in the context of real-world choices seems problematic, because it is not easy to purify the inter-personal impacts and give a straightforward interpretation. Especially at this stage, our objective is to test the learning effect in individuals' anticipation of congestion as well as the effect of recommendation. It is difficult, if not impossible, to have a situation that individuals repeatedly make decisions within a short time without disturbance of other possible changes in their environment. Therefore, we developed a controlled computer experiment to verify whether the proposed learning mechanism in the proposed model is valid. In this experiment, we deliberately simplify and abstract the structure corresponding to social reality in important particulars, explicitly excluding non-essential details. We manipulate experimental conditions and use the models of strategic behaviour to simulate other individuals' behaviour to imitate different scenarios. After collecting data, we checked the most influential factors and applied different expectation models to project anticipated congestion for the participants. By exploring which predictions are closest to the participants' choice behaviour in the laboratory, we gain insight into social interaction for which empirical real-world data should be used to estimate a more comprehensive model and make more accurate predictions.

This chapter is organized as follows. First, the research question concerning the properties of individual strategic choice behaviour in repeated situations is discussed. Next, the data collection, the experimental design, its implementation and process are described. This is followed by an analysis of the data. We first report some general results in terms of both performance and choice frequencies, and then describe the model estimates. Within the model estimates, first we examine a regression model that reflects the effects of the influential factors on subjects' performances. Then, we discuss the performances of the proposed choice models that are used to predict the expectation of subjects both in one-shot situations and in repeated situations, and the extent to which it is influenced by providing recommendation on the basis of different objectives. The chapter is completed with a conclusion and discussion section.

\subsection{Research question}

In strategic situations, various aspects and processes are assumed to simultaneously play an important role in individual's anticipation of congestion. Most of these relate to the presence of uncertainty of what others might do, such as how others adjust their behaviour under different types of recommendation, congestion aversion levels of the others and the uncertainty of others' preferences. Consistent with most behavioural theories, we expect that subjects improve their decisions in repeated choice situations, because they reduce the uncertainty in anticipated congestion by learning. Therefore, we investigated a set of research questions pertaining to how we expect each of these elements will affect individuals' decision making in strategic situations.

The first research question concerns how individuals respond in situations of repeated choice. We expect that the effects of recommendation, congestion aversion 
levels of others and the uncertainty of others' preferences may be different in situations of repeated choice compared to one-shot situations, as some of these factors may be strengthened as individuals learn. Moreover, as described in Chapter 5, reasoning in noisy introspection is based on iterative thinking with increasing error rates. Thus, we expect this model to perform better in predicting the expected number of other subjects in one-shot situations where subjects have no chance to learn what to anticipate. We expect individuals when making repeated choices under the same scenario settings learn how others make their decisions and therefore more often choose the best strategy. Consistent with this line of interpretation in empirical game theory, research in dynamic decisionmaking has also shown that in repeated choice situations individuals' learn from their past experience to reduce the uncertainty (e.g., Timmermans, et al., 2003; Arentze and Timmermans, 2005). Therefore, the reasoning underlying quantal response that is based on equilibrium could be closer in predicting the choices of others when individuals make their own strategic choices in repeated situations.

The second research question is related to how individuals respond to recommendations that are provided to achieve different underlying objectives in repeated situations. As indicated in the theory chapter, subjects are assumed to judge the value of the recommendation from their own interests and decide to follow it or not. In one-shot strategic situations, we might expect that because of the recommendation individuals not only have to consider how others anticipate congestion, but also have to anticipate how others respond to recommendation and adjust their behaviour accordingly. As a result, individual behaviour in situations with recommendations will likely differ from behaviour in situations without recommendations. Furthermore, in situations with recommendation when the choices are repeated, two possibilities may rise. On the one hand, the recommendation increases the complexity of the strategic reasoning since individuals have to take into account how others react in light of recommendation, which may disturb their learning process. On the other hand, if they expect that others follow the recommendation with a higher probability, the certainty of others' choices increases and the learning process of achieving a higher performance will be speed up because of less need to predict the equilibrium distribution. Moreover, different recommendation objectives may bring about different anticipated reactions and hence strategic behaviour

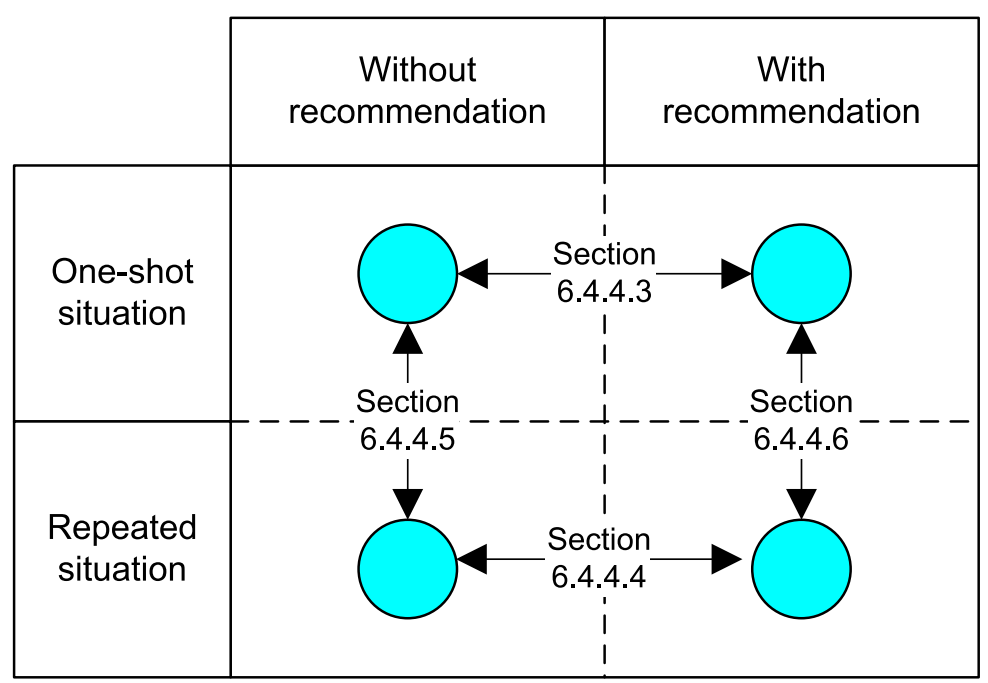

Figure 6-1 Structure of two dimensional effects 
of individuals. Thus, learning may be different when recommendations are provided. Figure 6-1 gives the structure of the two dimensional research questions.

In addition to this, some of influential factors may increase the complexity of the process of strategic reasoning. For instance, a lower congestion aversion level of others may cause a less accurate prediction of others' choices since others may deviate less from their original preference, whereas a higher uncertainty of others' preference may negatively influence the preciseness of the prediction of others' choices. Therefore, the combined effects of these factors are not straightforward.

\subsection{Repeated strategic choice data}

The experiment is designed to get insight into: (1) to what extent individuals improve their choices through learning in repeated interactions, and (2) how individuals make decisions when they have to take into account the decisions of others in two situations: with and without recommendation. We used an interactive computer experiment to monitor the changes in parameter settings to create typical scenarios that coincide with reality, and recorded the changes in subjects' choices regarding different combinations of parameter settings. Especially, we implemented our proposed model of equilibrium behaviour with a low error rate $(\mu=1.0)$ to simulate the choice behaviour of others that the subject encountered in the experiment to guarantee that identical choice scenarios are replicated in multiple rounds. The details of the algorithm used in the simulation are explained in Section 6.3.3. The simulated choice behaviour has the property that when close to a Nash equilibrium the best strategy is chosen with a probability that is close to 1 , reflecting the situation that the simulated players are perfectly rational with complete information about strategic interaction. We expect that this setting stimulates how real subjects learn.

\subsubsection{Data collection}

Data for the controlled computer experiment were collected in 2005 over four consecutive days from 10:00 am to 4:00 pm. Most of the participants were first year undergraduate students in the Department of Economic Psychology at Tilburg University and participated for course credit and a possible reward of ten euro cash based on their performance throughout the experiment. A total of 83 participants registered and participated. Participants were recruited by putting an announcement on the board, and signing up names on the attached registration form. Appendix B-1 shows the announcement. The experiment consisted largely of a computer game to gain as much points as possible. In the game, participants were asked to choose one out of three alternatives by using a mouse to click buttons on the computer screen. Table 6-1 gives an

Table 6-1 Overview of registration for repeated strategic choice

\begin{tabular}{|l|c|c|c|c|c|}
\hline & $12-04-2005$ & $13-04-2005$ & $14-04-2005$ & $15-04-2005$ & Total \\
\hline $10: 00 \mathrm{am}-4: 00 \mathrm{pm}$ & 26 & 27 & 18 & 12 & 83 \\
\hline
\end{tabular}


overview of the registration.

The experiments were run in a computer lab with limited cubical booths for 12 participants per session. Each session could last one hour, but depending on a subject's speed of decision making, they were free to use less time. No additional points were rewarded for a shorter consumption of time. They took, on average, 40 minutes to finish the experimental tasks. No subject appeared in more than one session. Communication between subjects was strictly forbidden before and during the experiment and, to the best of our knowledge, did not happen.

The experiment involved multiple tasks related to strategic behaviour. The possible application may cover a wide range of different problems. For instance, in the case of dinner timing choice for visiting a restaurant, the alternatives obviously refer to possible dinner times. Each of the alternatives has its default points, which can be interpreted as the original preference for a specific dinner time. With more individuals choosing the same alternative, a negative effect of waiting and crowdedness occurs due to congestion, and so, the points for that alternative decrease.

In the experiment, all subjects responded to all choice tasks. The specific scenarios that subjects faced within each task differed, depending on the manipulation of the parameter settings. The details of these manipulations and the underlying interpretation are explained in the following sections.

\subsubsection{Structure of the experimental design}

As mentioned before, differences in effects of various influential factors are of interest to investigate. Most of these are somewhat relevant for the uncertainty of anticipation about congestion. In this study, we investigated the four most relevant factors: (1) the effect of recommendation on others' choices, (2) uncertainty of others' preference, (3) the compliance rate of others, and (4) the marginal disutility of others for congestion. The latter three factors controlled for subjects' possible state of affairs regarding others' strategic choices in anticipation of congestion.

In a three-alternative choice task, based on different objectives, we can have four possible recommendation statues corresponding to recommending alternative $1,2,3$, and 'no recommendation'. The preference of others can vary from uncertain to certain. The following compliance rates of others were used $(10 \%, 20 \%, 30 \%$, and $0 \%$ for recommending alternative $1,2,3$ and no recommendation respectively). In addition, the compliance rates may also be moderately high $(+5 \%)$ or low $(-5 \%)$ for different situations. The marginal disutility of simulated other players could be the same as the subject is told to use (0.1) in making his choice or different (0.05). A full-factorial design was used to generate 32 scenarios of the combinations. The use of a full-factorial guaranteed that all main effects could be estimated independently from potential interaction effects. Appendix B-2 gives an overview of these 32 task profiles. To rule out as many unexpected influences as possible, we randomized the sequence of tasks. Within each task, there were multiple rounds of repeated choices.

Having multiple rounds is crucial in this study. Bearing in mind the complexity of the task, a few repetitions (e.g., five repeated choices for one task) will probably not be sufficient to analyze how subjects learn. For the purpose of the study, more repetitions will be better. On the other hand, after a certain number of repetitions, some boredom or 
loss of attention and concentration may drop in, leading to a reduced reliability of the measurements. Considering the complexity of the task and the results of pilot testing, it was therefore decided that 15 repeated choice situations is a good compromise. Thus, every subject made strategic choices for 15 repeated situations for each task.

\subsubsection{Implementation}

The three alternatives were assigned respectively 3, 5 and 7 utility points, assumed to represent their utility, unknown to the subjects. By using these core points, we were able to control the highest possible earning points across tasks. For every task, three random numbers were generated on the basis of these core points to show subjects the attractiveness of each alternative, called base-line points. The random number generations were based on a normal distribution with a mean vector set equal to these core points and a variance equal to 1 . This operation represents the population's fluctuation in average preference from time to time, situation by situation. Subjects were asked to use these points in assessing possible earnings.

In tasks without any recommendation, there was, of course, no compliance rate for the simulated players. As learned from the results of the numerical simulation in Chapter 4 , recommendations were linked to the objective of the information provider. That is, alternative 1 was recommended when the objective was equal usage, alternative 2 was recommended when the objective was welfare maximum, while alternative 3 was recommended when the objective was user equity.

In half of the tasks where the payoff is certain, both the subjects and the computer simulated players had the same preference as the points shown on the screen for each alternative. In the other half of the tasks, where there was uncertainty of the others' preference, the program generated a second set of random numbers to use as the preference for the simulated players. This second set of preference was unknown to the subjects. The subjects still used the points shown on the screen as their original preference for the different alternatives. The second set of random numbers was drawn from a normal distribution with a mean vector that is equal to the base-line points shown on the screen and a variance of 1 . This operation was intended to mimic the real life situation that not everyone has the same preference for the same thing, and an individual's preferences may deviate from the population average.

We used two levels $(0.1$ or 0.05$)$ for the marginal disutility of the simulated players. A marginal disutility of 0.1 is the same as the subject was told to use for assessing his possible earnings and making choice. Tasks with a simulated marginal disutility of others set to 0.05 and subjects using 0.1 were used to explore the situation that some individuals may dislike congestion more than others do.

The systematic self-expected payoff function for the simulated players was modified from equation (3.1) to: $E\left[\bar{U}_{j}\right]=U_{j}^{\text {preference }}-\theta\left((N-1) p_{j}^{*}+1\right)$, where $j=1,2,3$ and $N=100$. We used the following algorithm for the simulated 100 players, given the task settings including the assumed preference vector for each option, compliance rate for the recommendation, and the marginal disutility as described above:

1. A total of 100 sets of random values were drawn for the preference vector from a normal density distribution with an average means equal to the preference setting 
vector for the three options respectively and a variance equal to 1 . Every set defined a simulated player.

2. These values were used along with a marginal disutility of congestion $(0.1$ or 0.05 for each individual according to task settings) to calculate the probabilities that each individual will choose a certain option using equation (3.8). To calculate these probabilities, we used $\mu=1.0$ to approximate a Nash equilibrium behaviour.

3. The simulated probabilities were summed for each option across all players, generating a distribution of choices for 100 players in the 'no recommendation' situation.

4. In situations with recommendation, we used simplified ways to update choice probabilities for the simulated players. We first assigned the number of simulated players $(100 q)$ to the recommended option according to the compliance rate $(q)$ in the task settings. Then, we scaled down the distribution of choices for each option from 100 to the remainder $(100-100 q)$, and added the assigned number, generating a new distribution of choices for 100 simulated players in the "with recommendation" situation.

This distribution of choices was used to calculate the points earned by the subjects.

\subsubsection{Process}

At the start of each session of the experiment, each subject was assigned to an individual cubical booth with a computer that had pre-installed the programme and read instructions on the computer screen. Each subject was told through these computer instructions that (s)he was participating in a strategic choice experiment and informed about the objective of the experiment. Subjects were told that their task in the experiment was to make smart choices to earn as many points as possible. The earnings would be calculated from the total points earned during the experiment. They were also instructed that at end of the experiment, the scores of all participants would be compared, and that subjects scoring the best $10 \%$ would receive a reward of ten euros. They were also informed that the experiment would take about one hour. During the experiment, subjects were not allowed to speak or communicate with other participants. If they had a question while the experiment was going on, they were told to ask the instructor.

Subjects were told that they would play against a computer that simulated another 100 players and that the computer would use a behavioural model to mimic the behaviour of these players. Like their own choice, this model was based on the number of points attached to each option and the expected number of other players that would choose the option. Subjects were told that their decisions and those of the simulated other 100 players simultaneously would determine the amount earned. It was emphasised that the earned amount depends on both the choices of the subject and those of the simulated other players.

Subjects were informed that the experiment involved 32 tasks with 15 rounds each. They were asked to make a choice in each round. At the beginning of each task, they were informed about the default payoff for the three options. That was the maximum number of points they could possibly get for choosing that option. They could take that as 
base-line points. Then, each subject and the 100 simulated players made a choice simultaneously. The earning that each subject received was calculated on the basis of the alternative default payoff minus an amount that depended on how many simulated other players chose the same alternative. With each additional player that chose the same alternative as the subject did, the number of earned points for this choice decreased according to some fixed number of points. Subjects were advised to learn this amount through repeated choices within the task.

To understand the principle, subjects were informed about how this earning is calculated by the following example:

"Suppose that the option that you choose has a default payoff of 10 points. There are in total 60 players who choose the same option, and the number of points decreases by 0.1 with each additional person. Then,

$$
\text { Your payoff }=10-0.1 \times 60=4 \text { (points)" }
$$

Subjects were also told that in some tasks, the computer would give a recommended alternative, and that these recommendations were based on one of three objectives: maximizing the total payoff for all participants, generating equal payoff for everybody or generating an equal distribution across the three alternatives. Moreover, they were told that the recommendation was the same for each individual subject and the simulated other players, and that it may or may not help to earn more points. They were also informed that if every one followed the recommendation, the recommended alternative could be chosen by too many players, which would decrease the amount they could have earned. Therefore, they were suggested to figure out how others might respond to the recommendation to make a better choice. The full text of instruction is given in Appendix B-3.

In order to check whether a subject read the introduction carefully and understood the concept, before a subject really started completing the designed choice tasks, (s)he was requested to accomplish three preparation stages. In the first stage, every subject was asked to answer three questions that related to the maximum expected number of players that might choose an alternative, the length of the experiment, and the way of calculating earnings for making a choice. The answers of these questions were clearly expressed in the introduction. More specifically, these questions were:

1. How many simulated other players do you play against?

2. How many tasks do you need to finish?

3. Suppose that the option that you choose has 8 default payoff points. There are in total 40 players choosing the same option, and the number of points is decreased by 0.1 for each additional player. Your payoff then is?

The computer program checked the answers to these questions. If a subject did not answer all questions correctly, (s)he was requested to read the introduction again and try to find answers from there. Subjects were not allowed to move to the second stage of the experiment preparation until the right three answers were given.

In the second stage, subjects were offered tips for the experiment. The tips focused on the interdependency and the interaction of the choice tasks. An explanation was given 
of the default payoffs, the point system and the usefulness of the recommendations. In addition, a comparison example was given of how to make a choice based on expectations (See Appendix B-4 for the full text.) During the whole experiment subjects was able to review the tips whenever they wanted.

After reading the tips, in the third stage, every subject had two trial tasks to get used to the game. The trials used the same screen appearance as the real tasks, but applied different core points. When the subject finished the two trial tasks, (s)he was informed about the total number of points earned from the trials and the highest possible number of earned points to stimulate the incentive to do better in the real tasks. The number of points earned in the trial tasks was not carried over to the real tasks and was not recorded as useful data.

In addition to these instructions and trials, several other operations were implemented to help subjects concentrate on the task at hand and smoothly complete the experiment. Figure 6-2 shows a screen shot that subjects faced when making their choices. First, the task number and the round number was clearly shown at the top line of the screen, emphasized in red as the largest part of the text on the screen was in black. With the indication of "Your base-line points", the three options were shown in three columns. If there was a recommendation, it was shown right under the points for that option; otherwise there was an empty space. By clicking a radio button under each option, subjects could express their choice. Before confirming with the "OK" button, subjects were allowed to change their choice by clicking a different radio button, which automatically cancelled the previous choice. If the subject skipped making a choice and clicked the "OK" button, a dialogue box would pop up automatically to remind the subject. The program paused until the subject clicked a button choosing an option. After

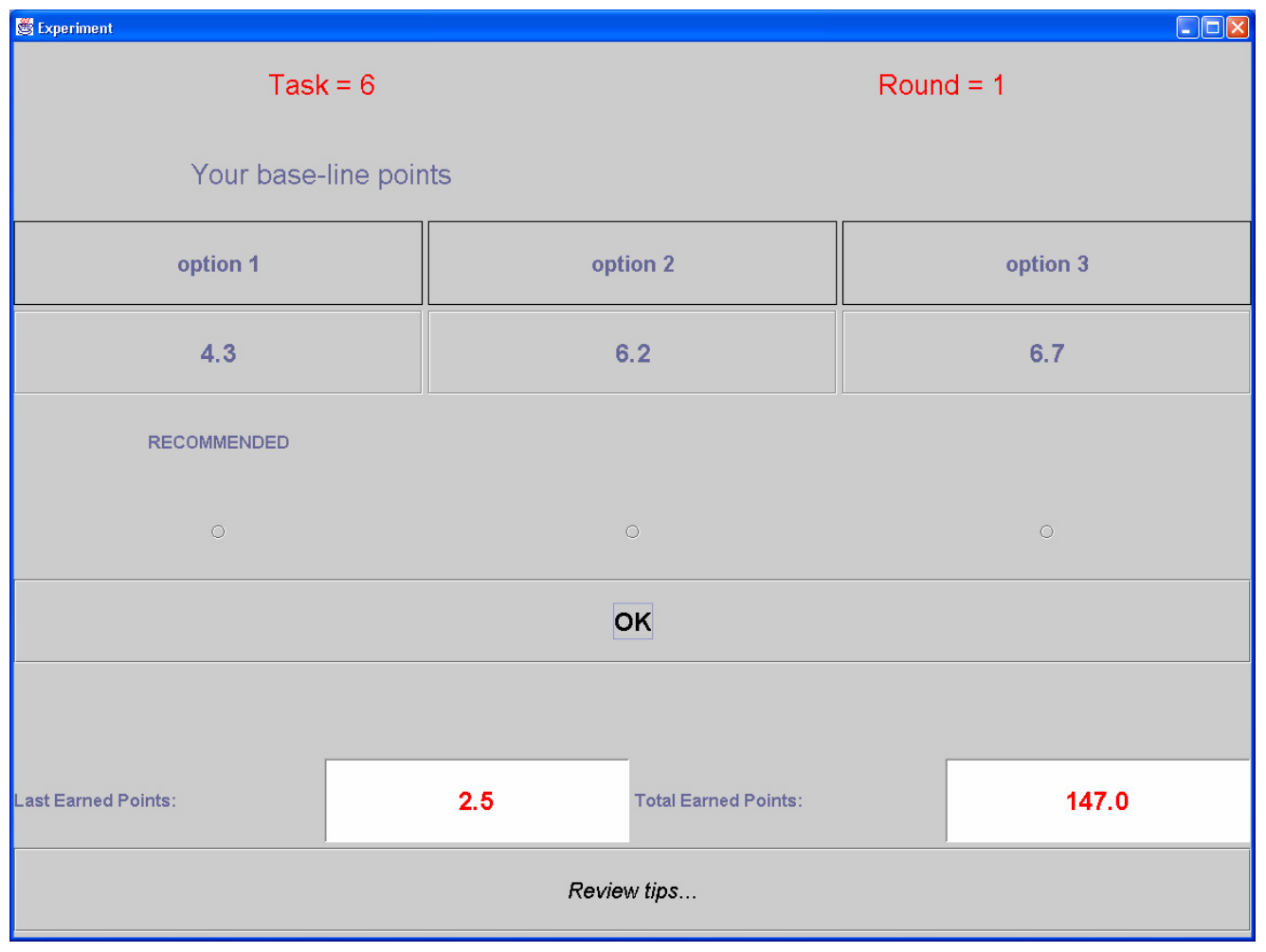

Figure 6-2 A screen shot of choice task 
clicking the "OK" button, the choice was finalized. The number of earned points was calculated on the basis of this choice and the data was recorded. The messages that include both the confirmation of the choice and how the number of earned points was calculated were shown under the "OK" button on the screen. Appendix B-5 gives an example of the screen shot after subjects made a choice. The number of earned points for the current choice was shown in red under the label "Last Earned Points", together with the number of "Total Earned Points" that summed across past rounds and tasks. The "Review Tips" button stayed at the bottom of the screen throughout the tasks for possible help.

Before a new task started a dialogue box pops up to draw the subject's attention that a new task would start and that the subject was required to modify his reasoning about the expected behaviour of others.

\subsection{Analysis of repeated strategic choice data}

To investigate our research questions, we used a random coefficients regression model to estimate the contribution of the various factors on the number of earned points and a random coefficient logit model to analyze the choice process. First, we will describe the results of the general statistics for both the performance and the choice frequencies, and then give a detailed explanation of methods used in the model analysis.

\subsubsection{General results: performance}

Each choice task has an ideal choice that would get the subject the maximum number of points that could be earned. Using this maximum as a reference level, the number of earned points of the actual choice that a subject made could be transformed into a

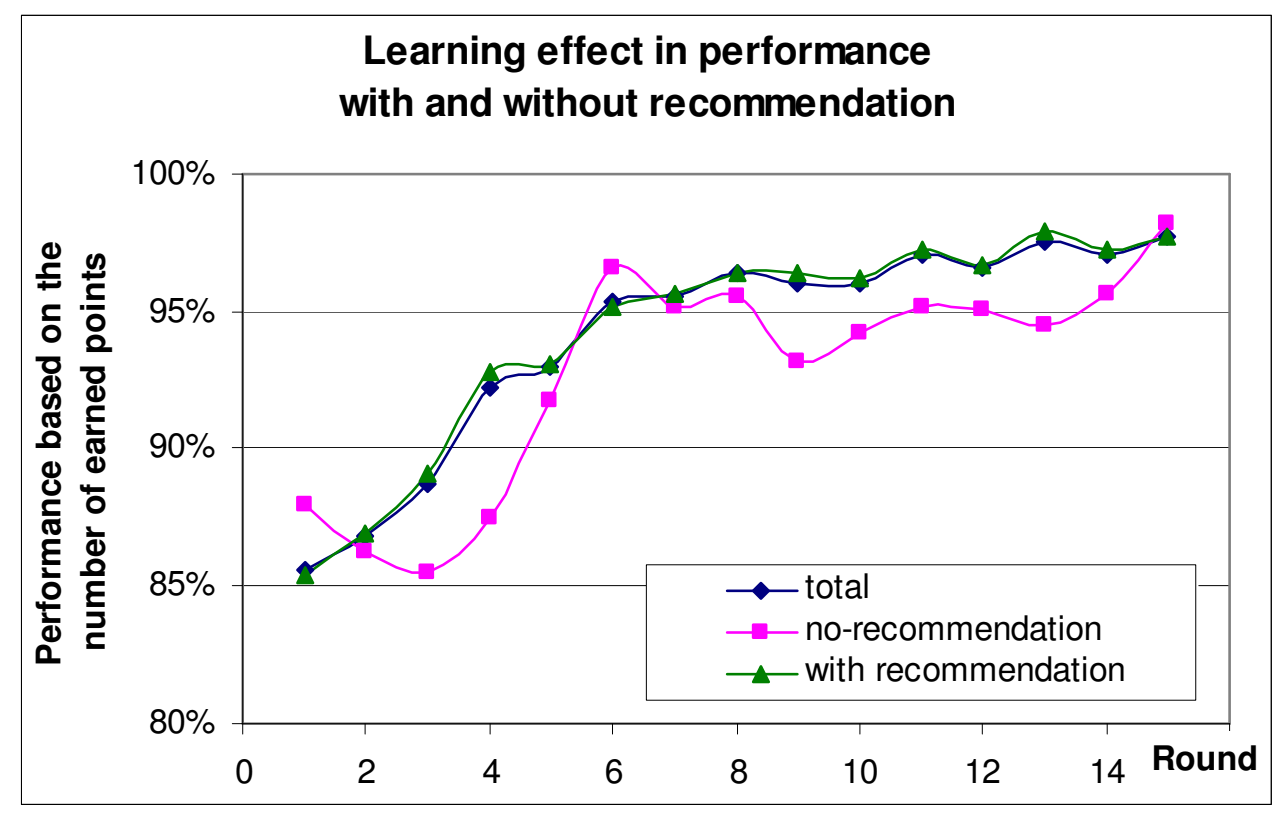

Figure 6-3 Average performance with and without recommendation 
performance indicator. The average total number of earned points across all subjects was 1178 points out of the maximum of 1320 points. Hence, the subjects achieved an average performance of $89.2 \%$. The highest individual performance was $94.1 \%$ and the lowest was $82.6 \%$ across the rounds and tasks. On average, subjects improved the performance from average $85.6 \%$ in round 1 across all tasks to $97.8 \%$ in round 15 .

Figure 6-3 plots the average performance achieved by all subjects across all tasks, differentiated for no recommendation tasks and with recommendation tasks. This trend line demonstrates a clear aggregate learning effect. There is a clear difference in behaviours between the tasks with recommendation and without recommendation. The average performance for the four tasks without recommendation was $92.8 \%$, which is lower than the average performance of $94.2 \%$ for the remaining 28 tasks with recommendation. Moreover, the increase from $87.9 \%$ in the first round to $98.2 \%$ in the $15^{\text {th }}$ round in tasks without recommendation is slightly less than the improvement from $85.4 \%$ to $97.7 \%$ in tasks with recommendation. With the recommendations, the performance illustrates a rather smooth improvement, while without recommendation, the performance shows some fluctuation.

There are a few possible reasons for this result. It could be that in case of tasks with recommendation subjects reduce the uncertainty of the choice behaviour of others by simply assuming others followed the recommendations. It could also be that since we set relatively high compliance rates for the simulated players, the recommended option is chosen too often and reduces the number of earned points. Subjects took the advantage of not following the recommendation to achieve more points and achieve a higher performance.

Among the tasks with recommendation, subjects received on average the highest performance $(95.3 \%)$ in tasks that linked the recommendations to the objective of user equity, followed by the objective of equal usage (93.7\%) and welfare maximum (93.6\%). Figure 6-4 plots the performance over the rounds for specific recommendation situations.

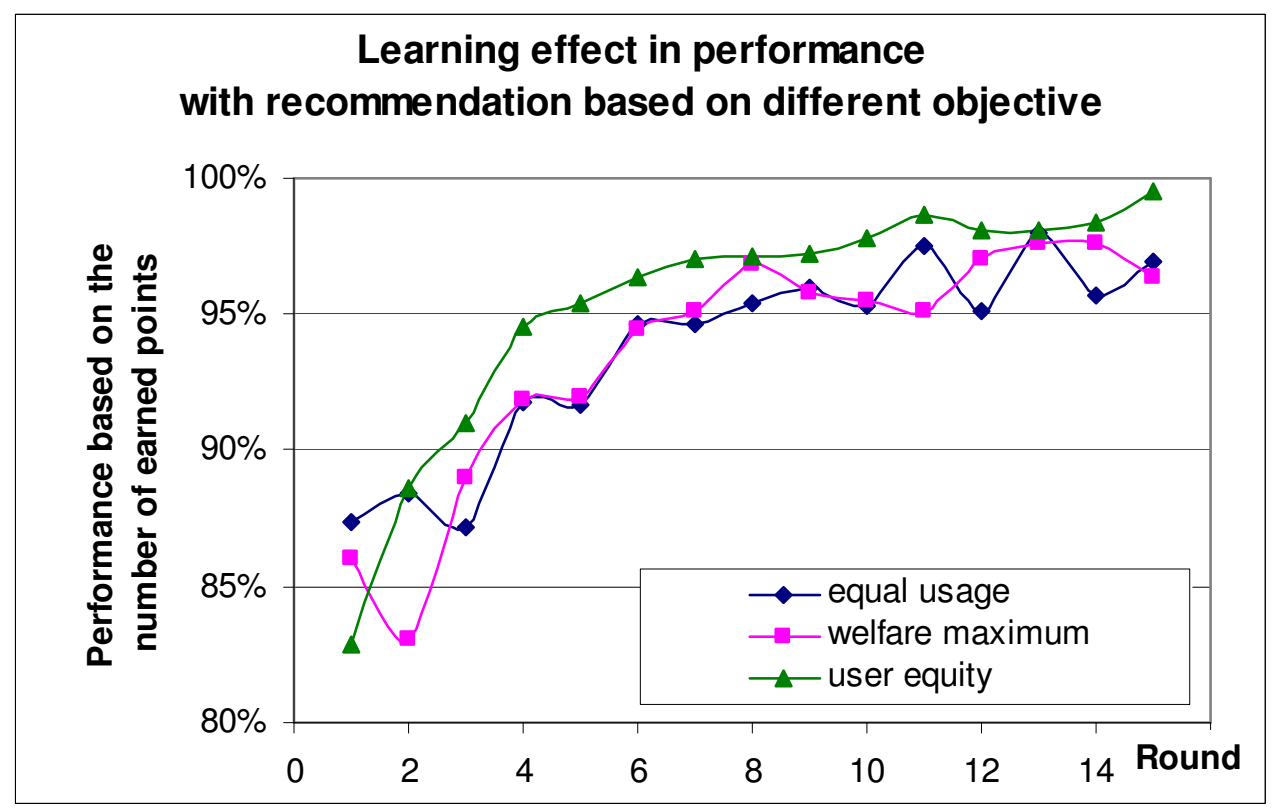

Figure 6-4 Average performance with recommendation for different objectives 
It also shows that, in tasks with recommendation based on different objectives, the objective of user equity has the largest improvement (from $82.9 \%$ in the $1^{\text {st }}$ round to $99.5 \%$ in the $15^{\text {th }}$ round) compared with the objective of equal usage (from $87.4 \%$ to $97 \%$ ) and welfare maximum (from $86 \%$ to $96.4 \%$ ).

This result is consistent with the reasoning that recommendations generated from different objectives have a different effect on individuals' choice behaviour. When the recommendation based on an objective that is not congruent but deviates from an individuals' interest, it will not assist individuals to do well in terms of personal performance. Hence, following this particular recommendation is not in one's selfinterest, resulting in fewer points. Although in this case, individual's performance is not as good as in cases where the recommendation is in line with a subject's interests such as user equity, it is still better than the case of no recommendation at all $(92.8 \%)$.

The highest average performance across rounds was $97.6 \%$ for task profile 32 (with recommendation on the basis of user equity, a marginal disutility of 0.1 , certain preference, and a $35 \%$ compliance rate). The lowest average performance was $87.2 \%$ for task profile 7 (with recommendation based on the objective of equal usage and a marginal disutility of 0.05 , but uncertain preference, and a $5 \%$ compliance rate). It is interesting to notice that the combination of the influential factors can make such a big difference in average performance. Taking a closer look at the average performance for each main factor, a more interesting picture evolves. The first column of Appendix B-2 gives the average performance value for each task profile.

Figure 6-5 plots average performance obtained by all subjects across all tasks, differentiated for tasks in which the preference of others is certain and tasks in which the preference of others is uncertain, and one randomly chosen subject's performance. The average performance for 16 tasks involving uncertainty about the preferences of others was $92.3 \%$, which is lower than the average performance of $95.8 \%$ for the other half of the tasks, involving no uncertainty about the preference of others. Figure 6-5 also

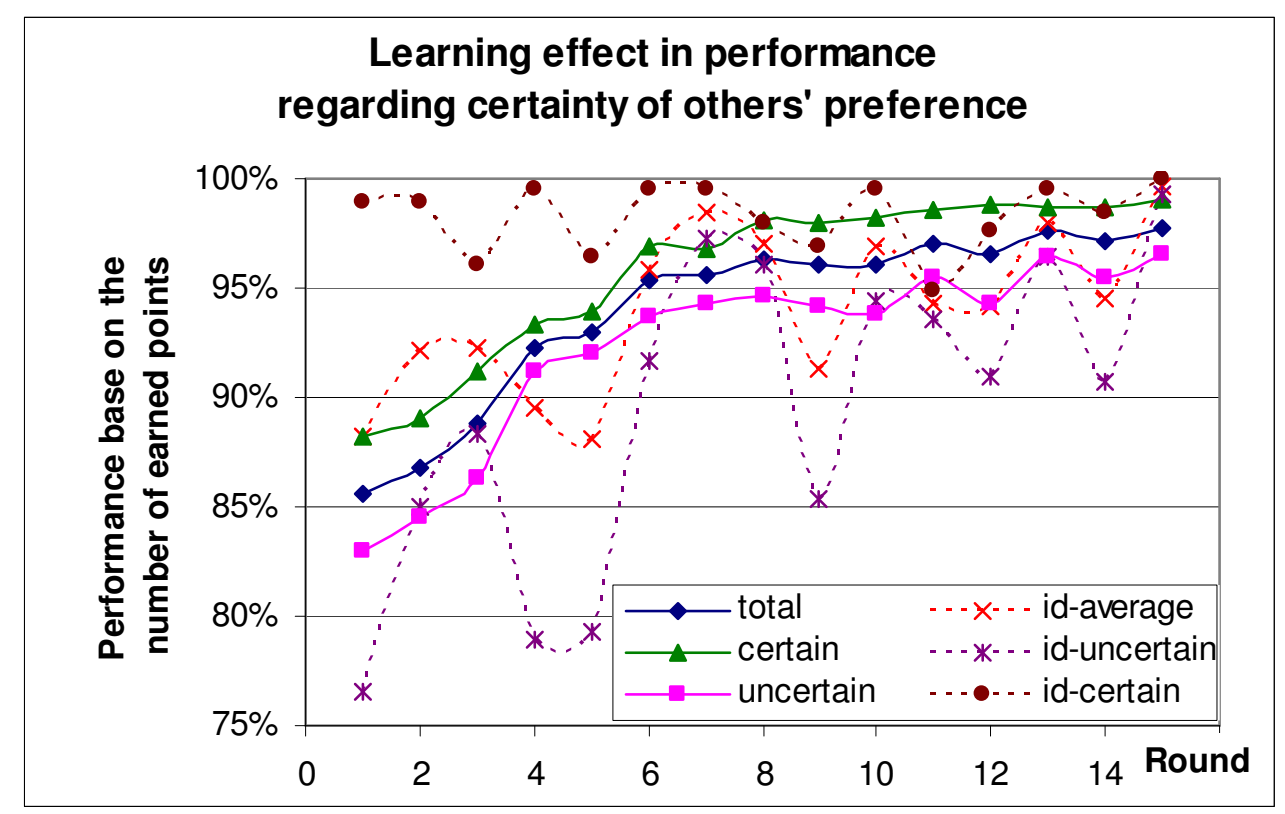

Figure 6-5 Average performance for the certainty of others' preference 
illustrates a clear difference between behaviour that involves uncertain preference of others and behaviour that involves certain preference. This is in line with our expectation that uncertainty about the preferences of others causes choice behaviour to contain more error and a lower probability (frequency) of choosing the best option. Without uncertainty, subjects' performance is rather stable; with uncertainty, subjects' performance fluctuates more. In addition, under conditions of uncertainty, there is evidence of more improvement in performance in early rounds compared to later rounds.

For tasks using a high marginal disutility of congestion $(\theta=0.1)$, the average performance was $93.7 \%$, while this average was $94.5 \%$ for tasks involving a low marginal disutility $(\theta=0.05)$, which is slightly better. The high marginal disutility of others means that others dislike congestion more, which indicates that they may deviate more from the original preference. Subjects could take the advantage of this overreaction by choosing the originally preferred one.

As for the effect of compliance rate, the higher the compliance rate, the better performance. With the compliance rate decreasing form $35 \%$ to respectively $25 \%, 15 \%$, $5 \%$, and 0 , the average performances also decrease from $95.8 \%$ to respectively $94.6 \%$, $93.6 \%, 93 \%$ and $92.8 \%$. It could be that with a high compliance rate for the simulated players, subjects took the advantage of not following the recommendation to receive more points and achieve better performance, since there would be too many players following the recommendation to choose the recommended option.

\subsubsection{General results: choice frequencies}

Figure 6-6 shows the average choice frequencies across 15 rounds for three options with core points 3,5 and 7 respectively. We used the core points only to calculate the probability of choosing an alternative when there are no strategic considerations and scaled to a total of 83 , the number of subjects who actually participated. We plotted the

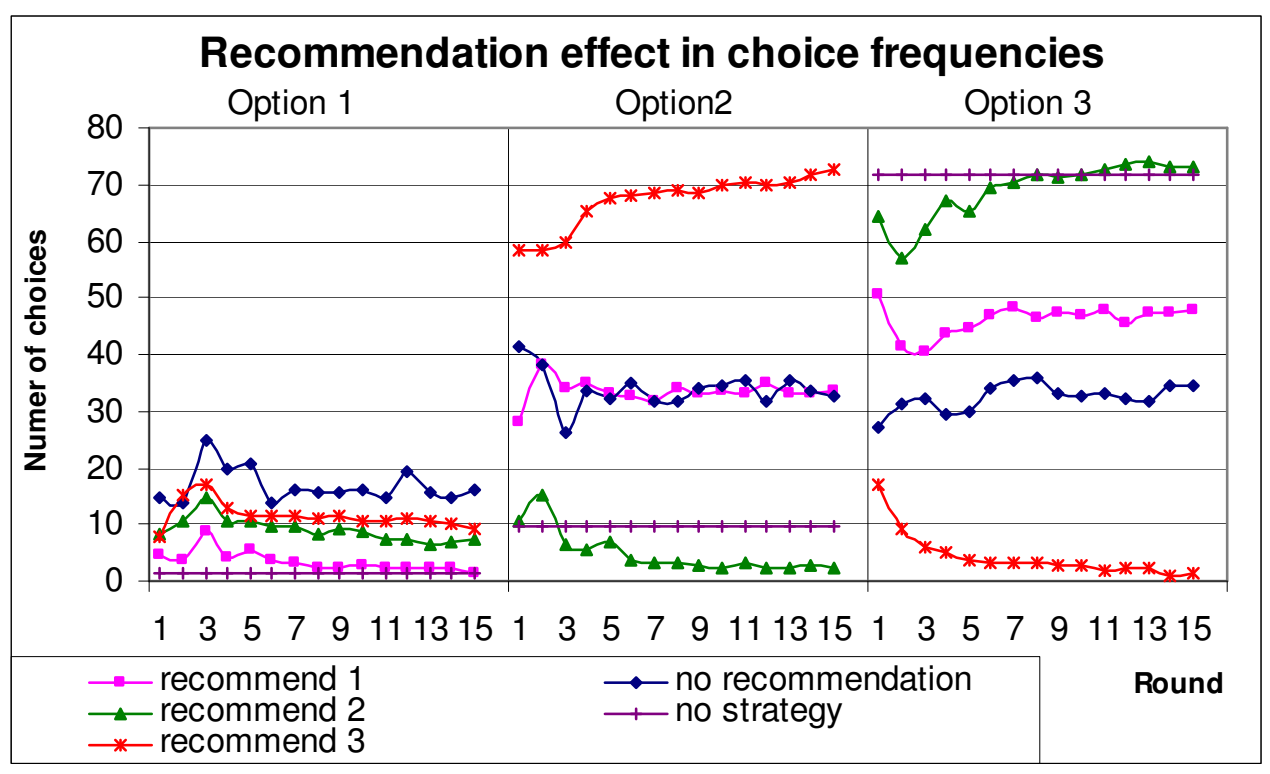

Figure 6-6 Recommendation effect in choice frequencies 
no strategic choice frequencies as a benchmark together with strategic choices that were observed in the experiment (the average choice frequencies for tasks without recommendation and for tasks with recommendation based on different underlying objectives). Figure 6-6 shows clear differences between the situations. First, with strategic considerations and no recommendation, the least preferred option 1 was chosen more often, the most preferred option 3 was chosen less often, and only slightly more than option 2. It seems that when subjects had to take into account others' choices, they were easily deviated from the most preferred alternative and turned to second best option, or even to the least preferred one.

Second, in tasks when one option is recommended, they chose that option less often by noticing or thinking that following the recommendation did not bring them good results, since that option might be chosen by too many others, following the recommendation. This finding supports the notion that individuals adjust their strategic anticipation of congestion in the light of recommendation because it indicates that they are concerned about whether or not to follow the recommendation, and how others might react to the recommendation.

Moreover, we observed an interesting circular effect of the recommendation. When option 1 was recommended, they chose option 3 more often. When option 2 was recommended, they changed to option 3 . But when option 3 was recommended, option 2 became the most attractive option to be chosen. These phenomenons reflect real life situations that information does not only have a direct influence, but also indirect effects. Therefore, the information provider should be aware of the consequence for the whole network when offering recommendations.

Figure 6-7 shows the average choice frequencies across 15 rounds for tasks involving uncertain preferences of others and tasks with certain preferences of others. In the certain preference tasks, subjects chose the option with the lowest default number of points less often than in the uncertain preference tasks. This suggests that subjects took the advantage of certainty to avoid the option that was least preferred (defined by the

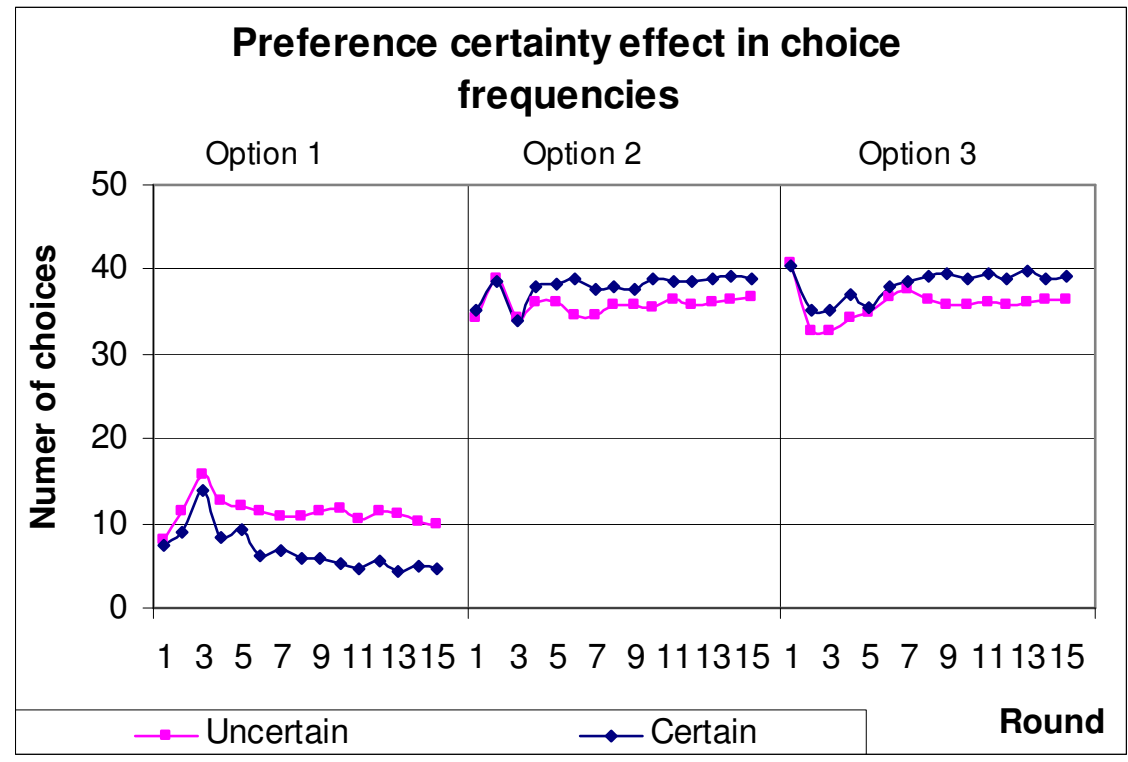

Figure 6-7 Preference certainty effect in choice frequencies 
lowest number of core points). In contrast, in tasks involving uncertain preference of others, subjects chose the two options that have a relative high preference (higher number of core points) less often. There is no significant difference in the choice frequencies for the two preferred options, both in certain preference tasks and uncertain preference tasks. This might imply that individuals focus on the most preferred options when they consider possible congestion; as a consequence they pick the second best alternative.

\subsubsection{Regression model estimation}

After exploring the general effects of recommendation, compliance rate, uncertainty of the preference of others and the marginal disutility of others regarding congestion, it was investigated whether the effect of these factors differs significantly in the number of earned points in early rounds compared to later rounds. From the observations, we noticed that some subjects for some reason showed sudden changes in their behaviour in the last two rounds. Therefore, we decided to use the $13^{\text {th }}$ round data for the comparison test with the $1^{\text {st }}$ round data.

To test for such difference, we first estimated a joint random coefficients linear regression model for the two rounds. The model structure also captures the repeated measures nature of the data, in which each subject responded to multiple choice tasks. The utility model for this regression model can be expressed as:

$$
\begin{aligned}
y_{i t} & =\alpha+\beta_{o p t 1}^{A} X_{o p t 1}+\beta_{o p t 2}^{A} X_{o p t 2}+\beta_{r e c 01}^{A} X_{r e c o 1}+\beta_{r e c 02}^{A} X_{r e c 02}+\beta_{r e c 03}^{A} X_{r e c o 3} \\
& +\beta_{c e r t}^{A} X_{c e r t}+\beta_{d s u}^{A} X_{d s u}+\beta_{o p t 1}^{B} X_{o p t 1}+\beta_{o p t 2}^{B} X_{o p t 2}+\beta_{r e c 01}^{B} X_{r e c o 1}+\beta_{r e c o 2}^{B} X_{r e c o 2} \\
& +\beta_{r e c 03}^{B} X_{r e c 03}+\beta_{c e r t}^{B} X_{c e r t}+\beta_{d s u}^{B} X_{d s u}+\varepsilon_{i t}+\mu_{i}
\end{aligned}
$$

where $y_{i t}$ is the number of earned points of subject $i$ when making choice $t$ in choice tasks; $\alpha$ is a constant term; $\varepsilon_{i t}$ is a normally distributed individual and option specific random component; $\mu_{i}$ is a normally distributed individual specific random component; $\boldsymbol{\beta}_{\text {opt }}^{A} \boldsymbol{X}_{\text {opt }}, \boldsymbol{\beta}_{\text {reco }}^{A} \boldsymbol{X}_{\text {reco }}, \boldsymbol{\beta}_{\text {cert }}^{A} \boldsymbol{X}_{\text {cert }}$ and $\boldsymbol{\beta}_{d s u}^{A} \boldsymbol{X}_{\text {dus }}$ are respectively the effect of the specific option-choice, recommendation, certainty of the preference of others, and the marginal disutility of others regarding congestion. "opt" are option choice dummies, the baseline is option 3. "reco" are recommendation dummies, the baseline is no recommendation. Superscript "A" denotes first round, whereas "B" denotes thirteenth round.

This random coefficients model is a special case with only the constant term specified to be random, and a generalized regression model, since the variance of the random term has the restriction: $E\left[\mu_{i}\right]=0, \operatorname{Var}\left[\mu_{i}\right]=\sigma_{\mu}^{2}, \operatorname{Cov}\left[\varepsilon_{i t}, \mu_{i}\right]=0$, and $\operatorname{Var}\left[\varepsilon_{i t}+\mu_{i}\right]=\sigma^{2}=\sigma_{\varepsilon}^{2}+\sigma_{\mu}^{2}$.

Table 6-2a presents the parameter estimates for comparing the $1^{\text {st }}$ round with the $13^{\text {th }}$ round of data. The number of observations is 5312 across 83 subjects, 32 tasks and 2 rounds. Table $6-2 \mathrm{~b}$ reports the corresponding statistics for the classical model test. The group effect here is reflected in individual-specific effects. The tables show increasing log likelihoods and $R^{2} \mathrm{~s}$, and decreasing sums of squares as we go through the classical 
Table 6-2a Regression model estimates ( $1^{\text {st }}$ round vs. $13^{\text {th }}$ round)

\begin{tabular}{|l|c|c|c|c|}
\hline & \multicolumn{2}{|c|}{$1^{\text {st }}$ round } & \multicolumn{2}{c|}{$13^{\text {th }}$ round } \\
\hline Attribute & Parameter & t-value & Parameter & t-value \\
\hline Intercept & $1.84^{*}$ & 60.08 & na & -11.53 \\
\hline Option 1 & $-0.47^{*}$ & -12.11 & $-0.53^{*}$ & -5.56 \\
\hline Option 2 & $0.14^{*}$ & 5.92 & $-0.17^{*}$ & 3.32 \\
\hline Recommendation 1 & 0.07 & 1.68 & $0.17^{*}$ & 2.39 \\
\hline Recommendation 2 & $0.13^{*}$ & 2.52 & $0.14^{*}$ & 0.14 \\
\hline Recommendation 3 & $-0.13^{*}$ & -5.12 & 0.01 & 2.98 \\
\hline Preference certainty & $0.13^{*}$ & 5.74 & $0.08^{*}$ & 14.46 \\
\hline Compliance rate & $2.26^{*}$ & 12.41 & $2.96^{*}$ & 5.37 \\
\hline Marginal disutility & -0.46 & -1.28 & $2.19^{*}$ & 2 \\
\hline
\end{tabular}

${ }^{*}$ significant at $\mathrm{p}<.05$ level; na $=$ not applicable.

models: (1) constant term only, (2) group effects only, (3) $\mathbf{X}$-variables only, and (4) $\mathbf{X}$ and group effects. This indicates that the regressors have been appropriately defined and are able to explain the data.

The first column in Table 6-2a presents the parameter estimates for the $1^{\text {st }}$ round. After the correction of alternative-specific preferences, two out of three recommendations, preference certainty and compliance rate are significant at the $95 \%$ significance level. The estimated effect of marginal disutility of others is not significantly different from 0 . Subjects show a clear ability to take into account most factors about how others might do when trying to earn a higher number of points even in the first round.

Table 6-2b Test statistics for the classical regression model

\begin{tabular}{|l|c|c|c|}
\hline Model & Log likelihood & Sum of squares & R-squared \\
\hline (1) Constant term only & -7675.60 & 5595.71 & 0 \\
\hline (2) Group effects only & -7616.94 & 5473.48 & 0.02 \\
\hline (3) $\mathbf{X}$-variables only & -7327.99 & 4918.57 & 0.12 \\
\hline (4) $\mathbf{X}$ and group effects & -7268.54 & 4800.60 & 0.14 \\
\hline
\end{tabular}


The second column in Table 6-2a presents the parameter estimates for the $13^{\text {th }}$ round. As in the $1^{\text {st }}$ round, after the correction of alternative-specific preferences, two out of three recommendations, preference certainty and compliance rate were significant at the $95 \%$ significance level. Effect estimates are somewhat different from those pertaining to the $1^{\text {st }}$ round. Moreover, the estimated effect of marginal disutility changed to be significant at the $95 \%$ level, which means it plays a role in the decision-making process. It seems that some of the effects are strengthened in the $13^{\text {th }}$ round.

Table 6-3 Contrast parameter estimates $\left(1^{\text {st }}\right.$ round vs. $13^{\text {th }}$ round $)$

\begin{tabular}{|c|c|c|c|c|c|}
\hline & & \multicolumn{2}{|c|}{ Data operation } & \multicolumn{2}{|c|}{ Estimated result } \\
\hline & Data set & $1^{\text {st }}$ round & $13^{\text {th }}$ round & Parameter & t-value \\
\hline & Intercept & & & $1.84^{*}$ & 60.07 \\
\hline \multirow{8}{*}{$\begin{array}{l}\text { Original } \\
\text { variables }\end{array}$} & Option 1 & & & $-0.50^{*}$ & -15.93 \\
\hline & Option 2 & & & -0.01 & -0.67 \\
\hline & Recommendation 1 & & & $0.12^{*}$ & 3.95 \\
\hline & Recommendation 2 & & & $0.13^{*}$ & 4.03 \\
\hline & Recommendation 3 & & & $-0.15^{*}$ & -3.77 \\
\hline & Preference certainty & & & $0.11^{*}$ & 7.02 \\
\hline & Compliance rate & & & $2.61^{*}$ & 22.53 \\
\hline & Marginal disutility & & & $0.86^{*}$ & 3.21 \\
\hline \multirow{8}{*}{$\begin{array}{l}\text { Contrast } \\
\text { variables }\end{array}$} & Option 1 contrast & \multirow{8}{*}{$\times(+1)$} & \multirow{8}{*}{$\times(-1)$} & 0.03 & 1.03 \\
\hline & Option 2 contrast & & & $0.16^{*}$ & 7.73 \\
\hline & Recommendation 1 contrast & & & -0.05 & -1.33 \\
\hline & Recommendation 2 contrast & & & -0.01 & -0.13 \\
\hline & Recommendation 3 contrast & & & $-0.16^{*}$ & -3.12 \\
\hline & Preference certainty contrast & & & 0.03 & 1.38 \\
\hline & Compliance rate contrast & & & $-0.35^{*}$ & -2.27 \\
\hline & Marginal disutility contrast & & & $-1.33^{*}$ & -4.80 \\
\hline
\end{tabular}

* significant at $\mathrm{p}<.05$ level. 
To test whether the effects of these influential factors differ significantly, the following analyses were conducted. For each pair of data sets an additional variable was created to measure the contrast between the parameters of two data sets. In the first data set, these contrast variables were multiplied by " +1 ", and thus are equal to the original variables, whereas for the second data set, these contrast variables were multiplied by " -1 ", and thus are equal to the negative values of the original data. If the corresponding contrast parameter is significantly different from zero, it can be concluded that the parameter is different for two data sets. In addition, we checked the scale difference of the two data sets to make sure that any significant difference is not caused by a scale factor.

In Table 6-3, the number of observations and the number of parameters to be estimated is still 5312 and 13 respectively. As Table 6-3 shows, differences in recommendation 1 and 2 effects are not significant between the $1^{\text {st }}$ and $13^{\text {th }}$ round. The effect of recommendation 3 is significantly different between the two rounds. The effect of preference certainty is significant, but not significantly different between the $1^{\text {st }}$ and $13^{\text {th }}$ round. The effect of others' compliance rate is significant, and significantly different between the two rounds. Difference in the effects of marginal disutility of congestion is significant. The effects of marginal disutility are significantly closer to 0 in the $1^{\text {st }}$ round compared to the 13th round (see Table 6-2a). In line with what one would expect if learning occurs, the effects of marginal disutility and compliance rate are strengthened as subjects learn to use them.

\subsubsection{Choice model estimation}

Having found that subjects learn how to use the marginal disutility and compliance rate of others with respect to congestion to improve the number of earned points, next the explanatory power of the proposed models for the anticipated effects of congestion were examined. The objective was: (1) to investigate whether the proposed strategic choice models are able of predicting the expected number of others choosing each option, and (2) to verify the hypothesis formulated in Chapter 5 - i.e., three models based on different levels of expectation are suitable for predicting behaviour in different situations - by comparing the estimates of the models with different predicted anticipations. That is, (a) their anticipation based on the noisy introspection model, (b) their anticipation based on a quantal response equilibrium, and (c) their anticipation based on a Nash equilibrium.

In this section, first the choice models that were used for estimation will be discussed. It will be followed by a discussion of how to predict the anticipated number of others for each subject and how to include it in the anticipated congestion term of the choice model. Then, the estimates of the choice data from the $1^{\text {st }}$ round will be reported in detail for the investigation of one-shot situations and from the $13^{\text {th }}$ round for repeated situations. Finally, the overall estimates across all rounds will be presented to examine learning in repeated situations both for tasks that do not involve any recommendations and for tasks involving recommendation.

\subsubsection{Strategic choice model}

A multinomial logit model (MNL) and a random coefficients logit model (RCL) that allows for heterogeneity in individuals' preferences for options were estimated (see 
equations 6.2 and 6.3). The model structure also captures the repeated measures nature of our data in which each subject responded to multiple choice tasks in multiple rounds. We first define the utility model for the no recommendation tasks:

$$
\begin{aligned}
& U_{i t}^{n o R}=\alpha_{i t}^{n o R}+\boldsymbol{\beta}_{\text {base }}^{n o R} \mathbf{X}_{\text {base }}+\boldsymbol{\beta}_{c o n}^{n o R} \mathbf{X}_{c o n}+\varepsilon_{i t k}^{n o R} \\
& \alpha_{i t}^{n o R}=\alpha^{n o R}+\left(\gamma_{\mathrm{t}}^{n o R}+\mathbf{v}_{\mathrm{it}}^{n o R}\right) \mathbf{X}_{\mathbf{t}}
\end{aligned}
$$

where $U_{i t}^{n o R}$ is subject $i$ 's utility of option $t$ in the no recommendation choice task; $\alpha_{i t}{ }^{n o R}$ is an individual and option specific random intercept, with average $\alpha^{\text {noR }}$, a option specific effect $\gamma_{t}^{n o R} \mathbf{X}_{t}$, and a normally distributed individual and option specific random component $\mathbf{v}_{\mathrm{it}}^{\text {noR }} ; \boldsymbol{\beta}_{\text {base }}^{n o R} \mathbf{X}_{\text {base }}$ and $\boldsymbol{\beta}_{c o n}^{n o R} \mathbf{X}_{c o n}$ are the effects of base-line points and anticipated congestion in the no recommendation choice tasks respectively, and $\varepsilon_{i t k}$ is an IID Gumbel distributed error term that is individual, option and observation $(k)$ specific. The utility model for the choice tasks with recommendation follows a similar structure:

$$
\begin{aligned}
& U_{i t}^{R}=\alpha_{i t}{ }^{R}+\boldsymbol{\beta}_{\text {base }}^{R} \mathbf{X}_{b a s e}+\boldsymbol{\beta}_{c o n}^{R} \mathbf{X}_{c o n}+\boldsymbol{\beta}_{\text {Reco1 }}^{R} \mathbf{X}_{\text {Reco1 }}+\boldsymbol{\beta}_{\text {Reco } 2}^{R} \mathbf{X}_{\text {Reco } 2}+\boldsymbol{\beta}_{\text {Reco3 }}^{R} \mathbf{X}_{R e c o 3}+\varepsilon_{i t k}^{R} \\
& \alpha_{i t}{ }^{R}=\alpha^{R}+\left(\gamma_{\mathrm{t}}^{R}+\mathbf{v}_{\mathrm{it}}^{R}\right) \mathbf{X}_{\mathrm{t}}
\end{aligned}
$$

where $\boldsymbol{\beta}_{\text {reco1 }}^{R} \mathbf{X}_{\text {recol }}, \boldsymbol{\beta}_{\text {reco2 }}^{R} \mathbf{X}_{\text {reco } 2}$ and $\boldsymbol{\beta}_{\text {reco3 }}^{R} \mathbf{X}_{\text {reco3 }}$ are effects of the recommendation for the three options respectively, and all other components are defined analogously to the no recommendation tasks.

In addition, it was investigated whether the effects of anticipated congestion differ significantly, depending on whether or not subjects need to take into account the reaction of others regarding recommendation (i.e., the effect of recommendations). To test for such differences in anticipated congestion across the two conditional tasks, an additional random coefficients logit model was estimated jointly across the two situations. This model allows for differences in the size of the random coefficients between the two situations and also includes an interaction of version with anticipated congestion to test for differences in effects of anticipated congestion between no recommendation tasks and with recommendation tasks. When this interaction is significant, it can be concluded that the effects of anticipated congestion are significantly affected by the additional complexity of providing recommendations in the strategic choice tasks. The model estimated to perform this test can be expressed as:

$$
\begin{aligned}
& U_{i t}=\alpha_{i t}+\boldsymbol{\beta}_{\text {base }} \mathbf{X}_{\text {base }}+\boldsymbol{\beta}_{c o n S} \mathbf{X}_{c o n S}+\boldsymbol{\beta}_{r e c o 1} \mathbf{X}_{r e c o 1}+\boldsymbol{\beta}_{r e c 02} \mathbf{X}_{r e c o 2}+\boldsymbol{\beta}_{r e c o 3} \mathbf{X}_{r e c o 3}+\varepsilon_{i t k} \\
& \beta_{c o n S} X_{c o n S}=\beta_{s t} X_{s t}+\left(\gamma_{\mathrm{t}}+\gamma_{s t} X_{s t}+\mathbf{v}_{i t s t}\right) \mathbf{X}_{c o n}
\end{aligned}
$$


where $\alpha_{i t}$ is an individual and option specific intercept; $\beta_{s t} X_{s t}$ is a main effect of task situation (no recommendation versus with recommendation) on the utility for a option, $\boldsymbol{\gamma}_{\mathrm{st}} X_{s t} \mathbf{X}_{c o n}$ is the interaction effect of task version and anticipated congestion, $\boldsymbol{v}_{\mathrm{itst}}$ is a normally distributed individual, option and task specific random component, and all other components are defined as before.

\subsubsection{Simulation for projecting the anticipated congestion}

Using the proposed strategic choice models as a starting point, the choices of others anticipated by subjects when making strategic decisions were predicted. To that effect, the expected choices of others were simulated from the viewpoint of each observed subject, based on the informed option preference vector (the points shown on the screen that subjects was told to use for making a choice in each task) and the marginal disutility used by the simulated players. More specifically, the following procedure was adopted:

1. A total of 100 set of random values were drawn for the preference vector, using a normal density distribution with a means, equal to that indicated on the screen with the label "your base-line points" and a variance of 1 . Every set defines an individual that subjects expected to encounter.

2. These values were used along with the marginal disutility settings of congestion in each task to calculate the probabilities that each individual, expected to be encountered, chooses a certain option. In this probability calculation, different values for $\mu$ were used, depending on the reasoning type (see equation 3.8). First, $\mu_{0}=4.4$ and $t=4.1$ were used, based on the study of Goeree and Holt (2004), to simulate the noisy introspection model, which assumes the lowest degree of rationality with increasing error in the anticipation reasoning. Second, following observations made by McKelvey and Palfrey (1995), the values $\mu_{0}=6.6$ and $t=1.0$ were implemented to simulate the quantal response equilibrium. This equilibrium reasoning has the property that each individual's choice probabilities are a logit stochastic response to the others. Third, since it is not possible for $\mu$ to take on a value of exactly zero in the simulation (as would be required in the Nash equilibrium), a value close to zero was used to approximate the resulting distribution of demand ( $\mu=1.0$ ), meaning that individuals play the best strategy with a probability that is very close to 1 .

3. The simulated probabilities for each option were summed across all individuals, generating a distribution of demand of 100 individuals.

4. These values were used as predicted anticipated congestion - the number of others choosing each option - projected for each subject.

\subsubsection{Results for one-shot situations}

Results for the strategic choices model estimation in the one-shot situations are presented in Table 6-4 using the projected congestion based on the Nash equilibrium and the data from the $1^{\text {st }}$ round. The first column of the table presents the parameter estimates for the situation of no recommendation. Four tasks were categorized in this situation. The overall 
fit of the model is not good, with an adjusted pseudo Rho-squared value of 0.12. The parameter of base-line points and one of the option specific parameter are significant at the $95 \%$ significance level. As expected, the parameter of base-line points positively influences utility. After correction for the effects of base-line points, subjects showed a clear tendency to choose option 2 and there is no significant difference in option specific preference between option 1 and option 3 . Interestingly, the effect of projected congestion is not significant.

The second column in Table 6-4 presents the parameter estimates for the situation with recommendation. A total of 28 tasks were included in this situation. The overall fit of the model is good, with an adjusted pseudo Rho-squared value of 0.36 . In this model, two of the recommendation parameters are significant at the $95 \%$ level together with the

Table 6-4 One-shot situation strategic choice model estimates $\left(1^{\text {st }} \text { round }\right)^{\S}$

\begin{tabular}{|c|c|c|c|c|}
\hline & \multicolumn{2}{|c|}{ No recommendation } & \multicolumn{2}{|c|}{ With recommendation } \\
\hline Attribute & parameter & t-value & parameter & $\mathrm{t}$-value \\
\hline Option 1 & 0.47 & 1.12 & $-0.62^{*}$ & -3.33 \\
\hline s.d. option 1 & $1.19^{*}$ & 3.63 & $0.82^{*}$ & 6.97 \\
\hline Option 2 & $1.12^{*}$ & 4.82 & $0.56^{*}$ & 4.37 \\
\hline s.d. option 2 & $0.74^{*}$ & 2.85 & $0.80^{*}$ & 7.75 \\
\hline Base points & $0.40^{*}$ & 2.18 & $0.57^{*}$ & 6.98 \\
\hline Anticipated congestion & $-0.003^{\mathrm{a}}$ & -0.19 & $-0.003^{\mathrm{a}}$ & -0.35 \\
\hline Recommendation 1 & na & & -0.36 & -0.19 \\
\hline Recommendation 2 & na & & $-1.51^{*}$ & -10.40 \\
\hline Recommendation 3 & na & & $-2.04^{*}$ & -16.70 \\
\hline Log likelihood null model & \multicolumn{2}{|c|}{-364.74} & \multicolumn{2}{|c|}{-2553.18} \\
\hline Log likelihood & \multicolumn{2}{|c|}{-319.09} & \multicolumn{2}{|c|}{-1636.23} \\
\hline Adj. McFadden R-sqr & \multicolumn{2}{|c|}{0.12} & \multicolumn{2}{|c|}{0.36} \\
\hline
\end{tabular}

$\S$ The number of observations in the "no recommendation" case is 332 , for 83 subjects in 4 tasks; the number of observation for the "with recommendation" case is 2324 , for 83 subjects in 28 tasks; na $=$ not applicable.

* significant at $\mathrm{p}<.05$ level.

a no recommendation estimate is significantly different from with recommendation choice task estimate at $\mathrm{p}<.05$ level. 
Table 6-5 One-shot situation MNL model log likelihoods ( $1^{\text {st }}$ round data)

\begin{tabular}{|l|c|c|c|}
\hline Situation & Nash equilibrium & Quantal response equilibrium & Noisy introspection \\
\hline No recommendation & -326.552 & -326.557 & -325.64 \\
(CAIC) & $(680.32)$ & $(680.34)$ & $(678.50)$ \\
\hline With recommendation & -1679.52 & -1679.02 & -1673.61 \\
(CAIC) & $(3420.30)$ & $(3419.30)$ & $(3408.48)$ \\
\hline
\end{tabular}

base-line points and the option specific parameters. The effect of recommending option 1 that has the lowest base-line points is not significant. As expected, the effect of base-line points is positive. Recommending option 2 brings about a negative effect as recommending option 3 does. Subjects showed a clear preference for choosing option 2, followed by option 3, and disliked choosing option 1 . The effect of projected congestion is also not significant in this case.

The results of the comparison test model (equation 6.4) show that the effects of the projected congestion of the two models are significantly different between the situations, despite the fact that they are not significantly impacting utility in each situation. This is evidenced by a significant interaction of situation condition and projected congestion.

The same estimation using the projected congestion base on the quantal response equilibrium and the noisy introspection model was made. Similar results are obtained with the exception that the effect of anticipated congestion based on the noisy introspection model is significant at the $95 \%$ level in situations with recommendation. With this additional explanatory parameter, the model using the noisy introspection model to predict anticipated congestion has a higher log likelihood value as shown in Table 6-5.

Table 6-5 shows results of log likelihoods for the MNL model using different projection in terms of anticipated congestion. Since all the other parameters used in the model estimation were the same, except for the parameter of the projected anticipation, the change in log likelihood directly reflects the explanatory power of the different projections.

In addition, the conventional Consistence Akaike Information Criterion (CAIC) statistics is reported. The CAIC calculation takes into account the statistical goodness of fit and the number of parameters that has to be estimated to achieve that degree of fit. Thus, the function could be expressed as: $Y=-2 L(\boldsymbol{\beta})+[\ln (N)+1] \times t$, where $L(\boldsymbol{\beta})$ is the $\log$ likelihood of the estimated model, $N$ is the number of observations and $t$ is the number of parameters. The model that produces the minimum value of these statistics is considered the most useful. In our case, the number of estimated parameters and the number of observations are the same for the three projection models, and the only difference between the models is the projected anticipation. Therefore, judgement of model performance based on the CAIC measure coincides with the log likelihood.

As shown in Table 6-5, the model with the projection based on the noisy introspection model has the highest log likelihood and the lowest CAIC value of the three projections both in the situation of no recommendation and the situation with 
recommendation. This result indicates that the model based on the noisy introspection model has more explanatory power for the first round data across all tasks. The model with the projection based on the quantal response equilibrium has a higher log likelihood in the situation with recommendation and a log likelihood in situation of no recommendation very close to that of the model using a projection based on the Nash equilibrium. This finding provides support for the hypothesis formulated in Chapter 5 that in one-shot situations, a quantal response model leads to more precise predictions of strategic choice behaviour compared to a Nash equilibrium model, but a model of noisy introspection predicts even better.

\subsubsection{Results for repeated situations}

The same estimation procedure was applied for all 15 rounds for both the situation of no recommendation and the situation with recommendation respectively. First, in this section, the detailed results using the $13^{\text {th }}$ round data are reported in Table 6-6 for the Nash equilibrium projection, together with a discussion that includes a comparison with the $1^{\text {st }}$ round results. Picking up the $13^{\text {th }}$ round data is to keep the consistency with the regression model that compared results using the $1^{\text {st }}$ round data with those of the $13^{\text {th }}$ round. Then, the general tend from the $1^{\text {st }}$ round to the $15^{\text {th }}$ round will be depicted for situations both with and without recommendation to investigate learning effects in the next section.

The first column of Table 6-6 presents the parameter estimates of the $13^{\text {th }}$ round data for the situation of no recommendation. The overall fit of the model is improved, with an adjusted pseudo Rho-squared value of 0.21 compared to a value of 0.12 in the $1^{\text {st }}$ round data. The parameter of base-line points and one of the option specific parameter are significant at the $95 \%$ significance level. As expected, the parameter of base-line points positively influences utility. After correcting for the positive effect of the base-line points, subjects showed a clear tendency to choose option 2 and there is no significant difference in preference between option 1 and option 3; the same as those derived from the $1^{\text {st }}$ round data. Interestingly, the effect of projected congestion is changed to significant at the $95 \%$ confidence level and the effect is negative, which suggests that the projected congestion has a negative effect on utility as expected. This change provides indirect evidence that subjects learned to use their anticipations of congestion when making a choice in repeated situations.

The second column in Table 6-6 presents the parameter estimates for the $13^{\text {th }}$ round data, involving 28 tasks with recommendation. The overall fit of the model is good, with an adjusted pseudo Rho-squared value of 0.52 , increasing from a value of 0.36 for the $1^{\text {st }}$ round data. In this estimation, the parameters of three recommendations are all significant at the $95 \%$ level together with the parameter of base-line points and the option specific parameter. The effect of recommending option 1 that has the lowest base-line points is changed to significantly negative although relatively small, while recommending option 2 brings about a bigger negative effect as does recommending option 3. Subjects showed a clear preference for choosing option 2 , followed by option 3 , and really disliked choosing option 1. The effect of projected congestion is changed to significant also in this case. But the negative effect of projected congestion is much smaller than the negative effect of recommendation, especially compared to the negative effects of recommending option 2 and recommending option 3 . 
Table 6-6 Repeated situation strategic choice model estimates $\left(13^{\text {th }} \text { round }\right)^{\S}$

\begin{tabular}{|c|c|c|c|c|}
\hline & \multicolumn{2}{|c|}{ No recommendation } & \multicolumn{2}{|c|}{ With recommendation } \\
\hline Attribute & parameter & t-value & parameter & t-value \\
\hline Option 1 & 0.41 & 1.23 & $-0.84^{*}$ & -5.13 \\
\hline s.d. option 1 & 0.31 & 0.52 & 0.24 & 1.19 \\
\hline Option 2 & $0.41^{*}$ & 2.08 & $0.28^{*}$ & 2.73 \\
\hline s.d. option 2 & 0.13 & 0.06 & 0.02 & 0.05 \\
\hline Base points & $2.11^{*}$ & 9.61 & $1.15^{*}$ & 11.37 \\
\hline Anticipated congestion & $-0.19^{* a}$ & -8.95 & $-0.07^{* \mathrm{a}}$ & -8.27 \\
\hline Recommendation 1 & na & & $-0.62^{*}$ & -2.77 \\
\hline Recommendation 2 & na & & $-3.19^{*}$ & -12.80 \\
\hline Recommendation 3 & na & & $-4.20^{*}$ & -17.55 \\
\hline Log likelihood null model & \multicolumn{2}{|c|}{-364.74} & \multicolumn{2}{|c|}{-2553.18} \\
\hline Log likelihood & \multicolumn{2}{|c|}{-284.41} & \multicolumn{2}{|c|}{-1235.46} \\
\hline Adj. McFadden R-sqr & \multicolumn{2}{|c|}{0.21} & \multicolumn{2}{|c|}{0.52} \\
\hline
\end{tabular}

$\S$ the number of observations for the "no recommendation" case is 332 , for 83 subjects in 4 tasks; the number of observations for the "with recommendation" case is 2324 , for 83 subjects in 28 tasks; na $=$ not applicable.

* significant at $\mathrm{p}<.05$ level.

${ }^{\text {a }}$ no recommendation estimate is significantly different from with recommendation choice task estimate at $\mathrm{p}<.05$ level.

The results of the comparison test (equation 6.4) show that effects of the projected congestion are significantly different between the two situations with and without recommendation as evidenced by a significant interaction of situation version and projected congestion. In line with what one would expected if recommendation is offered, the negative effects of projected congestion are significantly smaller for the situation with recommendation, possibly motivated by that fact that subjects' attention was distracted to the reaction of others towards recommendation. Their choices were more based on their expectations of others' compliance rate towards recommendation. As a consequence, they simplified the choice situation in some way, making the cognitive burden manageable.

The same procedure as described in Section 6.4.4.2 was applied to predict congestion based on the quantal response equilibrium and the noisy introspection model 


\section{Table 6-7 Repeated situation MNL model log likelihoods $\left(13^{\text {th }}\right.$ round data)}

\begin{tabular}{|l|c|c|c|}
\hline Situation & Nash equilibrium & Quantal response equilibrium & Noisy introspection \\
\hline No recommendation & -284.47 & -291.21 & -314.29 \\
(CAIC) & $(596.16)$ & $(609.64)$ & $(655.80)$ \\
\hline With recommendation & -1235.73 & -1225.40 & -1207.96 \\
(CAIC) & $(2532.72)$ & $(2512.06)$ & $(2477.18)$ \\
\hline
\end{tabular}

respectively. Similar estimation results for the parameter effects are found. Results of log likelihoods for the MNL model using different projection in terms of anticipated congestion for the $13^{\text {th }}$ round data are shown in Table 6-7. Since all other parameters used in the model estimation were the same, the change in the value of log likelihood directly reflects the explanatory power of the different projections. In addition, the CAIC statistics are also reported in Table 6-7 as an indication of model selection.

In the situation of no recommendation, the highest log likelihood value and the lowest CAIC value are obtained for the model with a projection based on the Nash equilibrium, followed by the model with the quantal response equilibrium projection. This result indicates that the model with the Nash equilibrium projection has more power in explaining subjects anticipated congestion for the $13^{\text {th }}$ round data in the situation of no recommendation. In other words, subjects noticed that others' choice influence the outcome of their own choice and they learned how to anticipate others' choices when they observed the choices of others in repeated play, implying their behaviour is closer to equilibrium behaviour with complete information and more similar to that of the simulated players that they played against.

Furthermore, in situations with recommendation, the model with the projection based on the noisy introspection model still has the highest log likelihood value in this later round. The model with the projection based on the quantal response equilibrium has a higher log likelihood value than the model with the projection based on the Nash equilibrium. This finding supports the notion that providing recommendation has an additional effect on subjects' reaction towards anticipated congestion. An explanation for this finding may be that recommendation adds an additional layer of complexity to the task, increasing the amount of error in the choice behaviour of the subjects. An alternative explanation may be that on the basis of expectations that others will follow the recommendation with a higher probability, the certainty of others' choices increases and the need of predicting equilibrium outcome decreases. In this way, recommendations are taken as an indicator of others' choices.

\subsubsection{Results about learning in situations without recommendation}

Figure 6-8 illustrates the log likelihood pattern based on estimating the model (equation 6.2) using the data over all rounds for the 4 tasks without recommendation, along with the projection for anticipated congestion based on the Nash equilibrium, the quantal response equilibrium, and the noisy introspection model. 
Since the independent variables are the same across rounds, the only difference is the endogenous dependent variable and, therefore, the estimated log likelihoods reflect the explanatory power regarding different projection bases for each round. Thus, the highest log likelihood value of one round indicates the best fitting model with specific projection for that round data. As a reference, we also estimated choice models without projecting anticipated congestion using the data across all rounds, but found that models without projection fit significantly worse compared to models with projection and that there are no significant differences in the log likelihood across the rounds for models without projection. Therefore, the results of models with projection are reported.

Figure 6-8 shows a clear increasing trend with some waving appearance for all three projection models. The log likelihood values increase from -326.55 in round 1 to -270.59 in round 15 for the Nash equilibrium model, from -326.56 to -276.76 for the quantal response model, and from -325.64 to -304.90 for the noisy introspection model. This result implies that in general the projection models gained more power in explaining subjects' anticipated congestion as the repetition of choice (round number) increases. This improvement also indicates that subjects' behaviour becomes closer to the proposed strategic choice models as they learn from repeated playing, and becomes more similar to that of the simulated players that they played against in the experiment.

There are a number of possible explanations for this waving phenomenon. First, one might suspect that during repeated play, subjects sometimes for some reason tried options. This type of fluctuation more often happen during the starting stage when people try to explore the environment. A second explanation may be that in response to repeated choice, subjects show some adaptive behaviour. When they notice that sticking on one option does not result in any increased utility, they get bored and start trying another option.

The eye-catching feature is the reversal in the order of the best-worst fitting models, when we move from round 1 to round 15 . The best fitting model for round 1 is the noisy introspection model, whereas the worst one is the quantal response equilibrium model,

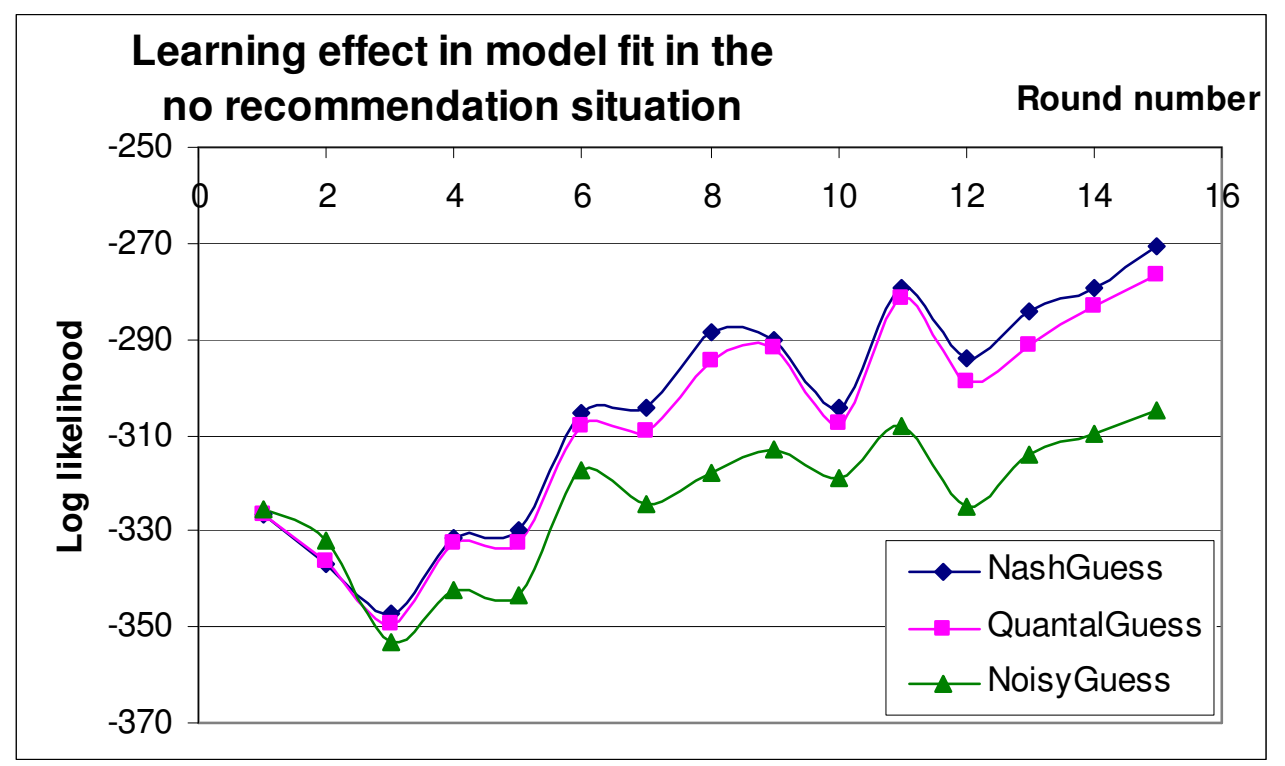

Figure 6-8 Learning effect in the no recommendation situation 
while the Nash equilibrium model is not significantly different from the latter model. The best fitting model for later rounds is the Nash equilibrium model, followed by respectively the quantal response equilibrium model, and the noisy introspection model. This finding supports the idea that individuals anticipate other individuals' choice and can anticipate more precisely through repeated observation, which is consistent with the theoretical properties of the proposed models in terms of the three levels of expectation of others' choices.

The analysis of the log likelihoods of the model estimates provides another interesting result that is worth mentioning. The differences in log likelihood between the random coefficients logit model and the multinomial logit model with the same projection decrease with increasing rounds. For example, for the Nash equilibrium model, the log likelihood for the RCL and the MNL model are respectively -326.55 and -319.09 in round 1 , and -270.59 and -270.39 in round 15. A possible explanation might be that heterogeneity in choices among the subjects was reduced as they learn in repeated situations that their anticipation of others becomes more and more similar.

\subsubsection{Results about learning in situations with recommendation}

Providing recommendation implies that the choice becomes more complicated. In tasks with recommendation, subjects have to adjust their anticipation. They have to make assumptions if and how others will adjust their behaviour given the recommendation. Accordingly, they may also improve their choice through observation in repeated situations. Figure 6-9 plots the pattern of log likelihoods for the various projection models using the data of all rounds for the 28 tasks with recommendation. As a reference, we also estimated choice models without projecting anticipated congestion using the data of all rounds, but found model fit significantly worse compared with models with projection. Also there is some improvement in model fit over rounds but not as significant as for the models with projection. Therefore, the results of models with projection are reported.

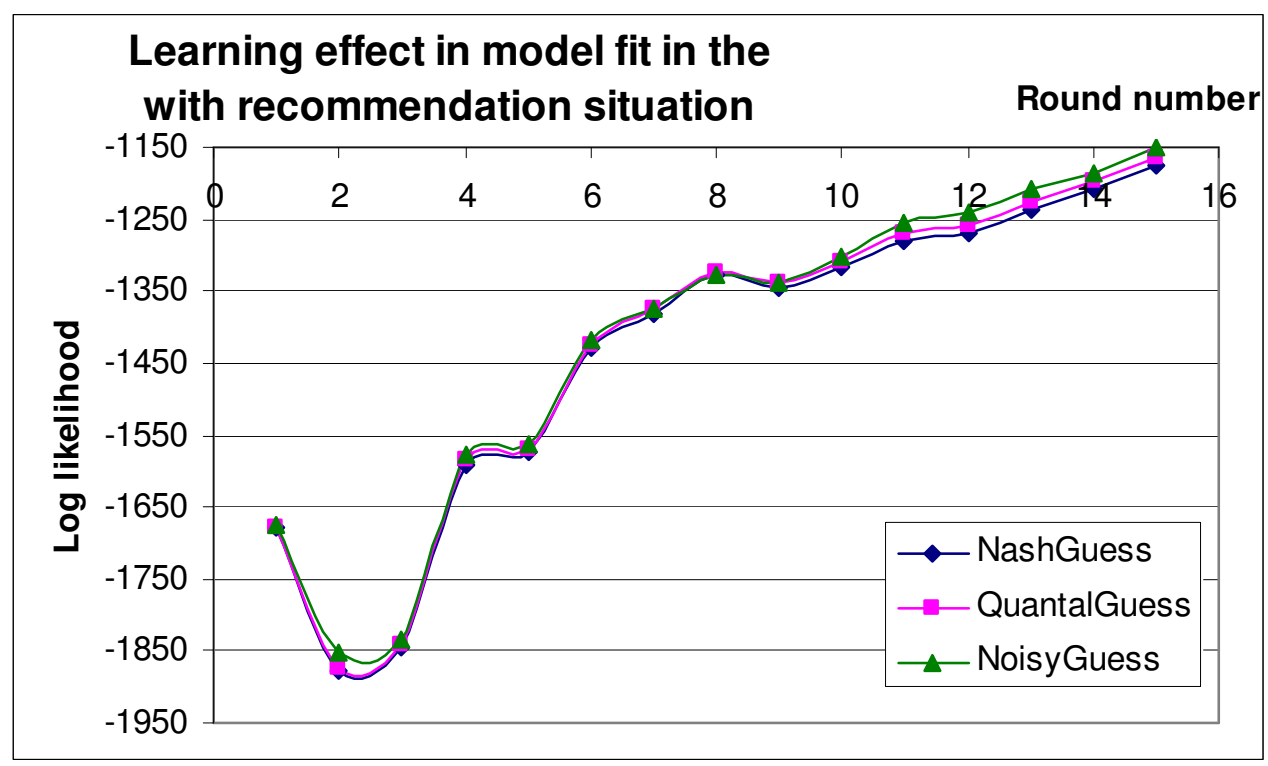

Figure 6-9 Learning effect in the with recommendation situation 
Figure 6-9 shows a clear increasing trend with some waving appearance for all three projection models, which is similar to the findings observed for the no recommendation situation. The log likelihood values increase from -1679.52 in round 1 to -1174.55 in round 15 for the Nash equilibrium model, from -1679.02 to -1163.78 for the quantal response model, and from -1673.61 to -1149.56 for the noisy introspection model. This result also shows that in general the explanatory power of the projection models increases with respect to subjects' anticipated congestion as the choice being repeated.

However, the major difference concerns the order of the best-worst fitting models. Unlike in no recommendation situation, the best fitting model in round 15 is still the noisy introspection model and the worst fitting model is the Nash equilibrium model. This indirectly reflects that the learning effect of projecting equilibrium outcome slows down compared with the no recommendation situation. This is not a surprise since multiple effects are possibly involved. First, one might suspect that with the additional layer of complexity by offering recommendation, subjects pay more attention to the reaction towards recommendation. That is, they focus more on how others might adjust their choice, and how their own choice should be adjusted accordingly. The negative effect of anticipated congestion is softened by the negative effect of recommendation (see Tables 6-4 and 6-6). As a result, one might suspect that with recommendations there is no or less need to predict the equilibrium outcomes, because the recommendations are already a good predictor of other players' choice behaviour. Therefore, the noisy introspection projection might be most accurate even in later rounds. Our observations on the model without projection showed partial evidence, since the log likelihood value of the model without projection also improved from $(-1679.58)$ in the $1^{\text {st }}$ round to $(-1209.47)$ in the $15^{\text {th }}$ round. However, the improvement was not as big as that for the model with projections.

Second, it might indicate that with this additional influential factor, subjects need longer time (more rounds) to learn how to deal with this additional complexity, implying more error in their decision making. Therefore, before they accomplish their learning, the quantal response equilibrium model that contains error will outperform the Nash equilibrium model that hardly has any error, and the noisy introspection model with unsure anticipation will do better than the quantal equilibrium model.

Third, when implementing the projection for the subjects, the reaction towards recommendation was not explicitly incorporated. Consequently, it is reasonable to expect that such projection assumed less error in decision making, which makes the model that is based on the Nash equilibrium less suitable for explaining subjects' choices in tasks with recommendation.

Consistent with the line of reasoning that learning slows down with increased task complexity, two sets of additional choice models were estimated for the tasks involving recommendation to examine: (1) the effect of marginal disutility of others and, (2) the effect of certainty about the preference of others.

First, to investigate the effect of marginal disutility of others, two models were estimated separately for the with recommendation situation: one using data of the tasks in which the marginal disutility of simulated players is 0.1 (the same as those of the real subjects), and the other using data of the tasks in which the marginal disutility of simulated players is 0.05 (different from the real subjects).

Figure 6-10 shows the resulting log likelihood patterns for the Nash equilibrium projection. It demonstrates a considerable improvement in model fit for both conditions, suggesting that learning took place. Similar pattern were found for the quantal response 


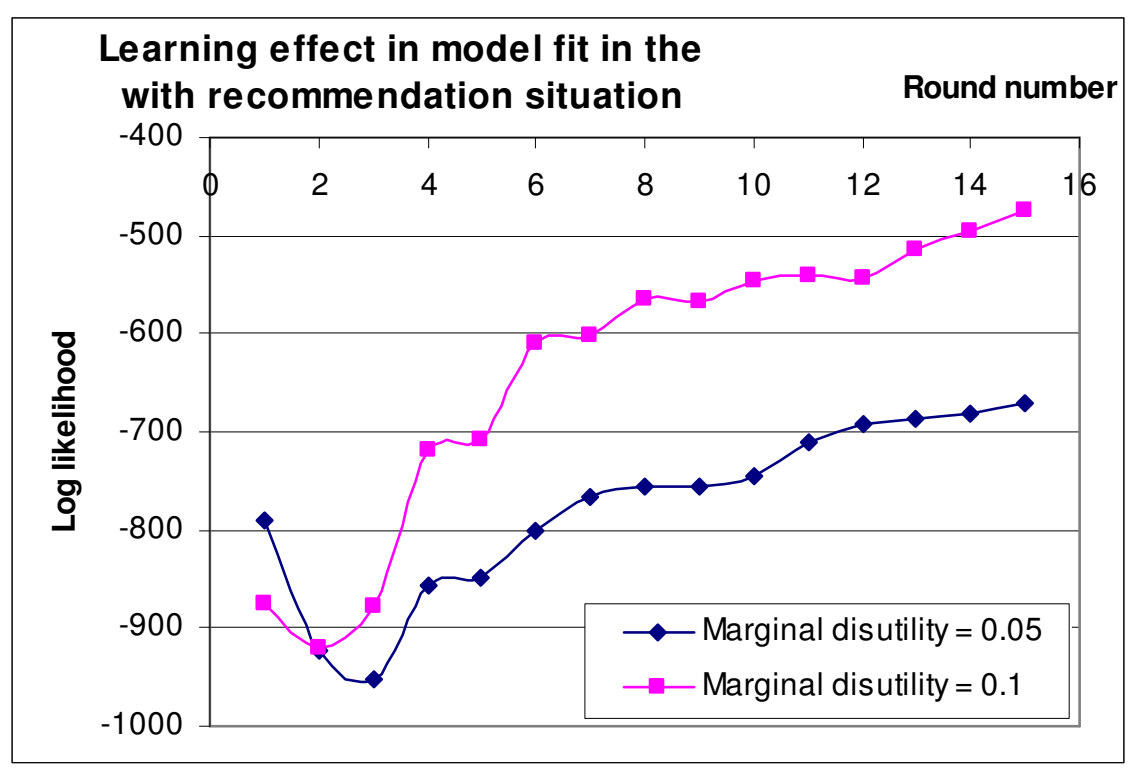

Figure 6-10 Learning effect for the marginal disutility of congestion

equilibrium and the noisy introspection model. Therefore, in the following only the results of the Nash equilibrium model are explored as an example.

Furthermore, results showed that for the first few rounds differences in model fit were relatively small across the two conditions. For subsequent rounds, differences started to become more visible. This could be interpreted as subjects making similar choices at the start in both conditions, but learnt differences through repeated play. When the marginal disutility of others is equal to 0.1 , the same as that of the real subjects used for calculating their utility, the choice task is less complex. Thus, learning is faster as evidenced by a bigger increase in the log likelihood value for the later rounds compared to the early rounds. With marginal disutility of others being equal to 0.05 , lower than that of real subjects, the choice task is more complicated. In this case, subjects need to figure out this marginal disutility value through repeated play, which decreases the speed of learning. Thus, the model based on the Nash equilibrium model has less explanatory power for predicting anticipated congestion in this case, which is reflected in less improvement in model fit, and a flatter appearance of the log likelihood for the later rounds.

Second, to investigate the effect of certainty of others' preference, two models were separately estimated for the with recommendation situation: one using data of the tasks that others' preference is certain using the same "base-line" points as that used by the real subjects, and the other using data of the tasks involving uncertainty about the preference of others.

Figure 6-11 illustrates the resulting log likelihood patterns for the Nash equilibrium projection. It shows an obvious improvement in model fit for both conditions, confirming that learning took place. Furthermore, model fit is lower for the tasks involving uncertainty about the preferences of others compared to the model for the tasks that involve no uncertainty. A straightforward explanation is that when uncertainty of others' preference is involved, the choice task is more difficult. Consequently, subjects take longer to correctly anticipation the degree of congestion. Thus, the projection based on 


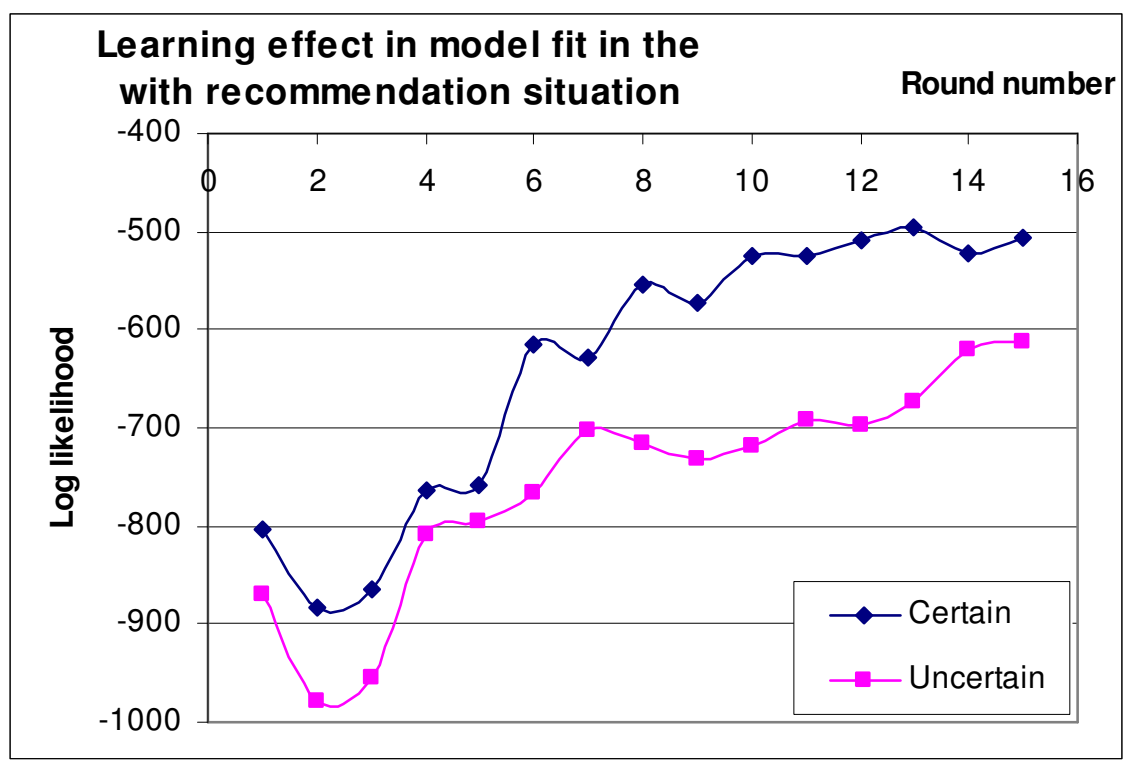

Figure 6-11 Learning effect for the certainty of others' preference

the Nash equilibrium model has less explanatory power for predicting anticipated congestion under conditions of uncertain preferences compared to conditions of certain preferences of others.

Similar estimates were derived for the quantal response equilibrium and the noisy introspection model. Because similar patterns were found, only the results obtained for the Nash equilibrium model are reported.

\subsection{Conclusion and discussion}

This study set out to address the question whether, and if so, how individuals incorporate strategic considerations with respect to congestion in their choices in repeated situations, and to what extent individuals' strategic choices may be influenced by providing recommendations. Based on the reported findings, the following conclusions can be drawn.

First, based on the analysis of performance, it can be concluded that individuals are able to anticipate the choices of other individuals, and learn to adjust their choices accordingly to avoid congestion and achieve better performance.

Second, based on the differences in choice frequencies regarding different task conditions, individuals behave differently depending on whether there is recommendation and on different objectives underlying the recommendation. With increased certainty, individuals stick more to their originally preferred options.

Third, based on the results of the regression model, the effects of marginal disutility of others and compliance rates of others are significantly strengthened in later rounds compared to early rounds as individuals learn to use them, while the effect of uncertainty of others' preference is not. 
Fourth, based on the strategic choice model for the $1^{\text {st }}$ round data, the noisy introspection model that involves iterative reasoning with an increasing error rate is most powerful in explaining individuals' strategic anticipation of congestion in one-shot situations both in situations of no recommendation and in situations with recommendation. This result provided support for our hypothesis.

Fifth, the results of the strategic choice model for the $1^{\text {st }}$ round and $13^{\text {th }}$ round data supports the notion that the effect of anticipated congestion differs significantly between no recommendation and with recommendation situations, since individuals are more concerned with how to react to the recommendation.

Sixth, by investigating the estimation of the strategic model across all rounds both in situations of no recommendation and in situations with recommendation, it can be concluded that the proposed model better predicts individuals' anticipation of congestion in later rounds as they learn what to expect.

Seventh, the underlying theory that the equilibrium model is more appropriate for predicting strategic choice in repeated situations is confirmed by the reversal in the order of the best-worst fitting models, when moving from round 1 to round 15 with data pertaining to the no recommendation situation.

Eighth, in situations with recommendation, individuals may use recommendation as an indirect indicator of others' choice behaviour, and hence, the need to predict equilibrium outcomes is weakened. Consequently, the noisy introspection model has a higher explanatory power in terms of individuals' strategic anticipation in repeated situations with recommendation.

Finally, by further investigating model fit, it can be concluded that the more complex the choice task, the slower the learning. When a recommendation is provided, a different marginal disutility and uncertainty about the preference of others are factors that increase the complexity of strategic choices. 


\section{Chapter 7}

\section{Conclusion and Discussion}

\subsection{Introduction}

Variation in demand over time can lead to congestion in capacity-constrained facilities. Under such circumstances, both service providers and individuals may act strategically since their decisions may affect each other. On the one hand, individuals care about what other individuals are likely to do so as to adjust their choices to avoid congestion. On the other hand, urban authorities and service providers need to anticipate the reactions of individuals when they provide information to influence individuals' behaviour in an attempt to achieve a more equal distribution of demand and reduce the potential burdens of congestion.

In this thesis, a modelling approach was developed that can assist urban authorities and service providers in predicting the strategic behaviour of individuals and evaluating the potential impact of information provision on demand distribution. This approach can help in better controlling the use of existing facilities. Methodologically, the proposed approach represents an integration of traditional discrete choice modelling and game theoretic interactive decision-making to capture the interaction and interdependencies both between individuals and between individuals and information providers.

This chapter summarizes the modelling efforts and reviews the most important findings. In addition, the strength and limitations of the proposed approach will be 
discussed. Finally, avenues of future research that represent promising extensions of the approach developed in this thesis will be proposed.

\subsection{Short summary of the study}

The proposed approach is based on a brief review of the relevant literature on models of consumer choice behaviour and game theory. Previous studies were discussed from three perspectives: individual choice models, choice behaviour in response to information, and choice behaviour in response to other individuals' behaviour. It is argued that most models of choice behaviour have in common the assumption that choices are based on a value judgement as a function of the external attributes. They lack the feedback mechanisms that count for the aggregate effect of individuals' choices. It is argued that such interdependence and interaction both between individuals and between individuals and information providers should be modelled based on interactive decision making. Therefore, game theory was introduced and a few applications were briefly mentioned. On the basis of this review, it was argued that combining the best features of the two research lines offers the most promising approach to develop an approach of modelling strategic behaviour in anticipation of congestion that tries to capture the interdependencies of individuals' choices.

Chapter 3 introduced the theoretical basis of strategic choice modelling. The interaction problem between individuals at the disaggregate level was formulated as a $\mathrm{N}$ player $J$-option game and uses a discrete choice model with an endogenous interaction term to describe the strategic choice behaviour of a given individual, in which the choices of other individuals are explanatory variables in the utility function. Three static stages of evolving behaviour in strategic situations were addressed: (1) expectation with complete information in ideal situations - Nash equilibrium, (2) expectation with incomplete information in repeated situations - quantal response equilibrium, and (3) expectation with incomplete information in one-shot situations - noisy introspection. The logit (exponential) probability response function was used to transform expectations of strategies into choice probabilities.

This model was further integrated to solve the interaction problem between an information provider and individuals at the aggregate level. By formulating a game of 2player 2-stages with a Stackelberg equilibrium, the information provider gets the leader's advantage without loosing the behavioural basis. Three typical roles of information providers were included: (1) a facility manager with the objective of equal usage across all time slots, (2) a government agency with the objective of welfare maximum across all individuals, and (3) a representative of collective individuals with the objective of user equity. It was argued that the developed strategic choice model can predict individuals' response to information provision.

The properties of the proposed model were tested through a series of numerical simulations, the results of which were reported in Chapter 4 . The results suggest that the proposed model is able to produce distinguishable choice behaviour when employing different values of parameters and the different results are easy interpretable in behavioural terms and consistent with intuitive expectations. Therefore, the support of face validity was provided. The simulation also explored the question whether the endogenously determined interaction may influence the distribution of individual's choices as well as the exogenous variables. The different levels of conjecture abilities 
underlying the decisions of individuals bring about an apparently different distribution. These distributions can be further transformed as a result of responses to the recommendations provided with the various objectives. The study indicated that differences in effects of these recommendations vary considerably with the information providers' objective.

Chapter 5 reported the first experiment about "timing choice" that examines the way individuals incorporate strategic considerations with respect to congestion in their timing decisions in one-shot situations. The experiment involved multiple tasks, covering two application contexts: (1) a hypothetical choice of dinner time while on vacation in London ("dinner timing choice"), and (2) a hypothetical and real-world choice of participation time in a research experiment ("participation timing choice"). The differences in dinner time preferences between "with strategic" and "without strategic consideration" suggested that individuals are able to anticipate other individuals' dinner timing choices, and indeed adjust their own timing choices accordingly to avoid waiting due to congestion. The differences in timing preference across the two context in nonstrategic situations as well as in strategic situations confirmed that individuals have similar preferences for activity participation times and dislike congestion, but have different distributions of preferred times for different activities. The shift in participation timing choices after receiving the specific information about the distribution of others proves that individuals adjust their strategic anticipation of congestion through information or experience about congestion. The analyses of the participation timing data provided mixed support for the approach. It provided evidence of the fact that in this realworld strategic situation individuals anticipate other individuals' timing choices. It showed that in this specific case individuals may not always take into account the congestion that they anticipate when making their own time choices.

To examine the effect of recommendation and learning in anticipation of congestion from one-shot to repeated situations in various scenarios, a second study on "strategic repeated choice" involving a controlled computer experiment with 32 tasks and 15 rounds each was conducted. Chapter 6 gave a detailed description of this study. The results showed that individuals are able to anticipate other individuals' choices, and did indeed learn to adjust their choices accordingly to avoid congestion. The differences in choice frequencies confirm that individuals behave differently depending on whether there is a recommendation, and on different objectives underlying the recommendation. With increased certainty, individuals stick more to their originally preferred options.

Comparing the effects of the influential factors in later rounds to early rounds, the effects of marginal disutility and compliance rate that others apply are significantly strengthened as individuals learn to use them, while the effect of uncertainty of others' preference is not. Based on the analyses of the strategic choice models for the 1 st round data, the noisy introspection model that involves iterative reasoning with an increasing error rate was most powerful in explaining individuals' strategic anticipation of congestion in one-shot situations, both in situations with and without recommendation. The results of the strategic choice models for the 1st round and the 13th round data provided support for the notion that the effect of anticipated congestion differs significantly between no recommendation and with recommendation situations, since individuals are more concerned with how to react to recommendation. By investigating model fit across all rounds in both situations, it could be concluded that the proposed approach better predicts individuals' anticipation of congestion in later rounds as they learn what to expect. The underlying theory that the equilibrium model is more 
appropriate for predicting strategic choice in repeated situations was confirmed by the reversal in the order of the best-worst fitting models, when moving from round 1 to round 15 with data pertaining to the no recommendation situation. In the situations with recommendation, individuals may use recommendation as an indicator of other's choice behaviour; and hence, the need to predict equilibrium outcome is weakened. Consequently, the noisy introspection model had a higher explanatory power in terms of individuals' strategic anticipation even in repeated situations. Also, it could be concluded that the more complex the choice task, the slower the learning. When a recommendation is provided, a different marginal disutility and uncertainty about the preference of others are the factors that increase the complexity of strategic choices.

Arguably, the most important conclusion that can be drawn from the study reported in this thesis is that individual's strategic choice behaviour that involves anticipation of others' choices should be modelled differently, especially in congested environments. Information providers that wish to influence the behaviour of individuals to optimize system use should take into account the extent to which individuals comply with the recommendation and individuals' anticipation of the extent to which other individuals will comply. The empirical studies show that the proposed approach effectively supports modelling these effects that are specifically related to the interdependent nature of people's behaviour in a congested environment.

\subsection{Discussion of the proposed modelling approach}

This thesis has described and exemplified the development and application of models of strategic choice behaviour. By highlighting the interdependent nature of people's behaviour in social and physical environments, it extends traditional research from a focus on individuals' response to an exogenous environment to the role of the endogenous interaction between different decision makers. It was shown that the proposed approach can represent the assumed interaction patterns both at the disaggregate level in response to other individuals' behaviour and at the aggregate level in response to information. Hence, it seems that the developed model is a potentially valuable tool to predict individuals' behaviour in strategic situations and, for information providers, to more effectively and efficiently achieve their objectives by choosing optimal guidance strategies while taking into account the reaction of individuals.

The aim of this project, however, was not to develop a fully operational, specific model, for a specific type of service, but rather to explore and investigate particular classes of strategic choice models. The focus is on model types that potentially could be further elaborated and applied to many different problems of strategic choice behaviour under conditions of uncertainty. Therefore, the developed approach is a general framework in the sense that it leaves open alternative specifications of the interaction components, and allows different decision styles: from perfect rational behaviour to completely random behaviour. In that sense, any application will require additional operational decisions, additional data collection and model estimation.

In modelling interaction between individuals, the generic form of the interaction term allows a flexible specification of the effect on utilities due to inter-individual interaction. This flexibility is two-fold. First, it can be defined using a variety of functions depending on the specific context that reflect the different computational complexity of the interaction impact. Second, it can involve positive effects as well as negative effects. 
For example, a positive effect due to economies of temporal agglomeration that favourable influence an individual's utility can be used to model choice leading to more efficient communication in work or school. Moreover, by an appropriate specification of the interaction term reflecting social-spatial structure, the model can also represent the positive feedback effect of copying behaviour under conformity pressure within certain social-spatial networks.

Furthermore, the solution to more complicated social dilemma problems that involved conflicts between self-interest and collective interest can be derived. In social dilemmas, people make choices with interdependent outcomes that have the following features: (1) the payoff to action based on self-interest (called defection) is higher than the payoff to action based on collective interest (called cooperation), regardless of what other individuals do, but (2) all individuals receive a lower payoff if all defect rather than cooperate. An example would be that air pollution decreases with an increasing number of car owners who use public transport instead of driving to work. The problem introduced in this thesis shares a common ground with social dilemmas in terms of choice interdependence, i.e., one person's action has consequences not only for himself but also for others. Modelling the interaction effect in social dilemmas, however, implies not only taking individual's consequences (e.g., personal costs) into account, but also group consequences. Thus, the interaction component of the proposed model needs to be modified appropriately to account for the additional complexity.

The proposed approach presents a behavioural model for the interaction between the information provider and individuals that allows one to determine optimal strategies of information provision. Although the present model only considers the optimal start time of an activity under strategic recommendation, the principles underlying the model can be extended to model any time-space interaction between the supply side and the demand side. This or a further elaborated version of the model might be especially valuable in situations involving control strategies, such as, for example, for guidance systems in route choice or decision support systems in urban tourism that help individuals to plan their day-trip while assisting in achieving some city-marketing objective. Most current systems either apply some algorithm with a weak behavioural basis to predict individuals' response to such information provision or use simple percentages of individuals complying with the recommendation. We argue that the inclusion of strategic interactive decision-making represents a better way of predicting individuals' response and evaluating the impact of the various policies.

\subsection{Avenues for future research}

Some possibilities for future research have already been mentioned in the previous section. This section extends this discussion and offers a more detailed perspective on the opportunities for extending the proposed approach towards more realistic and rich models in strategic situations.

The proposed approach is a mathematical modelling approach on a behaviour basis for analysing and predicting how humans behave in strategic situations. In the analyses, we assumed that all individuals are the same in terms of rationality. They have the same ability to think strategically and form beliefs based on analyses of what others might do. They have the same ability of reasoning and choosing the best response given those beliefs, and they adjust best responses and beliefs until they are mutually consistent, 
reaching equilibrium. It is easy to image that not every one behaves equally rationally in complex situations, so that assumptions are sometimes violated, implying that some individuals make more random choices than others do. Choices with some degree of irrationality in non-strategic situations may still be easy to model. But in strategic situations this is much more complicated, since the individuals' outcomes are intertwined. The presence of individuals, who do not think strategically or optimize their behaviour, even if there are very few of such individuals, can change what rational individuals should do. As a result, what a population of individuals is likely to do when some are not thinking strategically requires further research. To that effect the model needs to be expanded to accommodate heterogeneity with varying levels of rationality in the population. One way is to assume a population including both a group with imperfect expectations and a group with perfect expectations.

Moreover, in the current model, it is not explicitly addressed how inter-personal effects intertwine with personal constraints such as time budget, scheduling restrictions, and so on. To integrate resource limitations, the model can be further improved in a number of ways. Firstly, a different assumption about individuals' types may be useful, which should include the heterogeneity of individual constraints as well as the idiosyncratic errors. This would allow for covariance between one individual's evaluations, similar to a nested logit structure, but maintain the basic structure as described. Secondly, the heterogeneity of individual constraints could be incorporated into the utility function, which leads to different choice probabilities for each individual. Aggregating the probability across all individuals yields the expected number of individuals that choose each alternative. Within this avenue, making an early distinction among individual types may be useful, and may make the model computationally tractable.

In the current model, the scale parameter $\mu$ that is inversely related to error variance was used to reflect expectation uncertainty. However, it is possible that individuals have different certainty about the exogenous component and the endogenous component. They may be relatively more certain about their own preference, while less certain about the choice behaviour of others. Thus, rather than correcting the entire tradeoff for differences in the scale parameter $\mu$ (as is done in the proposed model) perhaps parameters for strategically influenced variables (such as congestion) should be corrected differently from the parameters of external variables (such as preference). Such an approach would change the relative impact of interaction compare to its impact in the current model.

How individuals process recommendations is a very complex decision making process. In the current study, it was assumed that the information users are the same in terms of the way they rationally react to the recommendation. It is easy to image that not every one behaves equally rationally in response to recommendation. A more comprehensive model could include three categories of information users: unguided user, imperfectly guided user, and perfectly guided user. Furthermore, such adjustment may be sensitive to different framing of recommendation and loss-aversion, as implied by risk attitude (Kahneman, and Tversky, 1982; Prelec, 2000). In general, people show anomalies and contradictions when facing risk and uncertainty. They may weight the displeasure associated with the loss more than the pleasure associated with the same amount of gain. Therefore, people may more easily adopt an advice involving gain than one implying loss. Integrating risk attitude in the modelling approach would add realism and richness to the current model (Han, et al., 2005). 
In the current approach, three static stages of strategic behaviour, which are capable in predicting the different steady-state distributions (i.e. in one-shot situations or repeated situations) and how these distributions shift in response to changes in exogenous parameters, were proposed. It does not explicitly address how strategic behaviour in oneshot situations, which is best modelled by a noisy introspection model, evolves into behaviour in repeated situations that is best modelled by a quantal equilibrium model or even a Nash equilibrium model. To model the dynamic process, an interactive learning approach that views equilibrium as the long-run outcome of a dynamic process of adjustment will be a promising approach. In strategic situations, individuals learn from changes in expectation based on (in)direct observations. That is, individuals not only learn and adjust their choice from their experiences with the chosen option, but also from the extent to which their expectations about the behaviour of others are consistent with actual choices.

In the current approach, for the demand side, we assumed a sub-game and a representative for the collective set of individuals, who distributed the flow to the urban facility. For the supply side, we assumed only one individual, who has different objectives based on the role he represents. It is also possible to formulate a sub-game on the supply side as well, which involves urban authority, property developers, retailers, etc. They will go though a negotiation process, so called "bargain" in game theory, in which different parties or protagonists try and reach agreement on the objectives. Then, a representative can derive the optimal strategies based on this mutual objective. Recent work by Arentze, et al. (2005) offers interesting insights in the possibilities for future research along this line. 


\section{Bibliography}

Adler, J. and Blue, V. (1998) Towards the design of intelligent traveller information systems, Transportation Research Part C: Emerging Technologies, 6(3), 157-172.

Ajzen, I. (1991) The theory of planned behaviour, Organizational Behaviour and Human Decision Processes, 50 (2), 179-211.

Alba, J., Lynch, J., Weitz, B., Janiszewski, C., Lutz, R., Sawyer, A. and Wood, S. (1997) Interactive home shopping: consumer, retailer, and manufacturer incentives to participate in electronic marketplaces, Journal of Marketing, 61(3), 38-53.

Anderson, L. and Holt, C.A. (1997) Information cascades in the laboratory, American Economic Review, 87(5), 847-862.

Aoki, M. (1995) Economic fluctuation with interactive agents: dynamic and stochastic externalities, Japanese Economic Review, 46(2), 148-165.

Arentze, T.A., Oppewal, H. and Timmermans, H.J.P. (2005) A multipurpose shopping trip model to assess retail agglomeration effects, Journal of Marketing Research, 42(1), 109-115.

Arentze, T.A., Sun, Z. and Timmermans, H.J.P. (2004) Modelling the dynamic use and impact of travel information on activity-travel (re)scheduling decisions, Paper presented at the Conference on Progress in Activity-Based Analysis, Maastricht, The Netherlands. 
Arentze, T. and Timmermans, H.J.P. (2000) Albatross: A Learning Based Transportation Oriented Simulation System, European Institute of Retailing and Services Studies, Eindhoven, The Netherlands.

Arentze, T.A. and Timmermans, H.J.P. (2003) A multi-agent model of negotiation processes between multiple actors in urban developments: a framework and results of numerical experiments, Environment and Planning B: Planning and Design, 30(3), 391-410.

Arentze, T.A. and Timmermans, H.J.P. (2004a) A theoretical framework for modelling activity-travel scheduling decisions in non-stationary environments under conditions of uncertainty and learning, Paper presented at the Conference on Progress in Activity-Based Analysis, Maastricht, The Netherlands.

Arentze, T.A. and Timmermans, H.J.P. (2004b) Capturing the role of awareness and information search processes on choice set formation in models of activity-travel behaviour, Paper presented at the $83^{\text {rd }}$ Annual Meeting of the Transportation Research Board, Washington, D.C., USA.

Arentze, T.A. and Timmermans, H.J.P. (2005) Modelling learning and adaptation in transportation contexts, Transportmetrica, 1(1), 13-22.

Arnott, R.A. Palma, A. de and Lindsey, R. (1991) Does providing information to road drivers reduce congestion? Transportation Research Part A: Policy and Practice, 25(5), 309-318.

Arthur, W.B. (1994), Inductive reasoning and bounded rationality, American Economic Review, 84(2), 406-411.

Bajari, P. and Kahn, M. (2001) Why do blacks live in cities and whites live in suburbs? mimeo, Stanford University, USA.

Barfield, W. and Dingus, T. (1998) Human Factors in Intelligent Transportation Systems, Lawrence Erlbaum Associates, Mahwah, NJ, USA.

Bargeman, B., Joh, C.-H. and Timmermans, H.J.P. (2002) A typology of tourist vacation behaviour using a sequence alignment method, Annals of Tourism Research, 29(2), 320-337.

Basar, T. and Olsder, G.J. (1995) Dynamic Non-cooperative Game Theory, Society for Industrial and Applied Mathematics, Philadelphia, PA, USA. [2 ${ }^{\text {nd }}$ ed., 1999]

Baxter, M. and Ewing, G. (1981) Models of recreational trip distribution, Regional Studies, 15(5), 327-344.

Bayer, P., McMillan, R. and Rueben, K. (2004) Residential segregation in general equilibrium, working paper No.885, Economic Growth Center, Yale University, USA.

Ben-Akiva, M., Bottom, J. and Ramming, M.S. (2001) Route guidance and information system, Institution of Mechanical Engineers, Proceedings, Part I: Journal of Systems and Control Engineering, 215(14), 317-324.

Ben-Akiva, M., Palma, A. de and Kaysi, I. (1991) Dynamic network models and driver information systems, Transportation Research Part A: Policy and Practice, 25(5), 251-266

Ben-Akiva, M. and Lerman, S.R. (1985) Discrete Choice Analysis: Theory and 
Application to Travel Demand, MIT Press, Cambridge, Massachusetts, London, UK, [2 $2^{\text {nd }}$ ed., 1987].

Ben-Akiva, M., McFadden, D., Gärling, T., Gopinath, D., Walker, J., Bolduc, D., BorschSupan, A., Delquie, P., Larichev, O., Morikawa, T., Polydoropoulou, A. and Rao, V. (1999) Extended Framework for modelling choice behaviour, Marketing Letters, 10(3), 187-202.

Bhat, C.R. and Koppelman, F.S. (1993) A conceptual framework of individual activity program generation, Transportation Research Part A: Policy and Practice, 27(6), 433-446.

Blume, L. and Durlauf, S. (2002) Equilibrium concepts for social interaction models, SSRI working papers, Social system research institute, University of Wisconsin, Madison, USA.

Borgers, A.W.J., Hofman, F. and Timmermans, H.J.P. (2002) Conditional choice modelling of time allocation among spouses in transport settings, The European Journal of Transport and Infrastructure Research, 2, 5-17.

Borgers, A.W.J., Timmermans, H.J.P. and Waerden, P.J.H.J. van der (2002) PATRICIA: predicting activity-travel interdependencies with a suite of choice-based, interlinked analyses, Transportation Research Record, 1807, 145-153.

Bowman, J.L. and Ben-Akiva, M.E. (1999) The day activity schedule approach to travel demand analysis, Paper presented at the $78^{\text {th }}$ Annual Meeting of the Transportation Research Board, Washington, D.C., USA.

Brewer, A.M. and Hensher, D.A. (2000) Distributed work and travel behaviour: the dynamics of interactive agency choices between employers and employees, Transportation, 27(1), 117-148.

Brock, W. and Durlauf, S. (2001) Discrete choice with social interactions, Review of Economic Studies, 68(2), 235-260.

Capra, C.M., Goeree, J.K., Gomez, R. and Holt, C.A. (1999) Anomalous behaviour in a traveller's dilemma, American Economic Review, 89(3), 678-690.

Chen, J. (1998) Integration of Dynamic Traffic Control and Assignment, Ph.D. Dissertation, Massachusetts Institute of Technology, Cambridge, MA, USA.

Chen, R.B. and Mahmassani, H.S. (2004) Travel time perception and learning mechanisms in traffic networks, Paper presented at the $83^{\text {rd }}$ Annual Meeting of the Transportation Research Board, Washington, D.C., USA.

Chorus, C.G., Molin, E.J.E. and Wee, G.P. van (2004) Use and effects of next-generation ATIS: a conceptual framework, Paper presented at the Conference on ActivityBased Analysis, Maastricht, The Netherlands.

Cramer, J.S. (2003) Logit Models From Economics and Other Fields, Cambridge University Press, Cambridge, UK.

Crouch, G.I. and Louviere, J.J. (2000) A review of choice modelling research in tourism, hospitality and leisure, Tourism Analysis, 5(2-4), 97-104.

Dellaert, B.G.C., Arentze, T.A., Borgers, A.W.J., Timmermans, H.J.P. and Bierlaire, M. (1998) A conjoint-based multi-purpose multi-stop model of consumer shopping centre choice, Journal of Marketing Research, 35(2), 177-188. 
Dellaert, B.G.C., Borgers, A.W.J. and Timmermans, H.J.P. (1996) Conjoint choice models of joint participation and activity choice, International Journal of Research in Marketing, 13(3), 251-264.

Dellaert, B.G.C., Borgers, A.W.J. and Timmermans, H.J.P. (1997a) Conjoint models of tourist portfolio choice: theory and illustration, Leisure Sciences, 19, 31-58.

Dellaert, B.G.C., Borgers, A.W.J. and Timmermans, H.J.P. (1997b) Consumer activity pattern choice: development and test of stage dependent conjoint choice experiments, Journal of Retailing and Consumer Services, 4, 25-38.

Dellaert, B.G.C. and Stremersch, S. (2003) Modelling the consumer decision to mass customise, Proceedings of the 2003 World Congress on Mass Customisation and Personalization, Munich, Germany.

Dugundji, E.R. and Gulyas, L. (2003) An exploration of the role of global versus local and social versus spatial networks in transportation mode choice behaviour in The Netherlands, Proceedings of AGENT 2003: Challenges in Social Simulation, University of Chicago, Chicago, USA.

Dugundji, E.R. and Walker, J.L. (2005) Discrete choice with social and spatial network interdependencies: an empirical example using mixed GEV models with field and "panel" effects, Paper presented at the $84^{\text {th }}$ Annual Meeting of the Transportation Research Board, Washington, D.C., USA.

Emmerink, R.H.M., Axhausen, K. W., Nijkamp, P. and Rietveld, P. (1995) Effects of information in road transport networks with recurrent congestion, Transportation, 22(1), 21-53.

Engemann, A., Radtke, M. and Sachs, S. (1989) A computer simulation system for individual decision processes, in: Upmeyer, A. (ed.) Attitudes and Behavioural Decisions, Springer-Verlag, New York, USA, 125-149.

Ettema, D.F., Borgers, A.W.J. and Timmermans, H.J.P. (2000) A simulation model of activity scheduling heuristics: an empirical test, Geographical and Environmental Modelling, 4, 175-187.

Fehr, E. and Fischbacher, U. (2002) Why social preference matter - the impact of nonselfish motive on competition, cooperation and incentives, The Economic Journal, 112, C1-C33.

Fehr, E. and Schmidt, K.M. (1999) A theory of fairness, competition and cooperation, The Quarterly Journal of Economics, 114(3), 817-868.

Fleischmann, M, Hall, J.M. and Pyke, D.F. (2004) Smart pricing, MIT Sloan Management Review, 45(2), 9-13.

Fotheringham, A. S. (1983) A new set of spatial interaction models: the theory of competing destinations, Environment and Planning A, 15(1), 15-36.

Fotheringham, A.S. and Wong, D.W.S. (1991) The modifiable areal unit problem in multivariate statistical analysis, Environment and Planning A, 23(7), 1025-1044.

Fuddenberg, D. and Levine, D. (1998) The Theory of Learning in Games, MIT Press, Cambridge, MA, USA.

Fudenberg, D. and Tirole, J. (1991) Game Theory, MIT Press, Cambridge, MA, USA. 
Gärling, T., Brännäs, K., Garvill, J., Golledge, R.G., Gopal, S., Holm, E. and Lindberg, E. (1989) Household activity scheduling, in: Transportation Policy, Management and Technology Towards 2001: Selected Proceedings of the Fifth World Conference on Transport Research, 4, Western Periodicals, Ventura, CA, 235-248.

Gärling, T., Kalen, T., Romanus, J., Selart, M. and Vilhelmson, B. (1998) Computer simulation of household activity scheduling, Environment and Planning A, 30(4), 665-679.

Gliebe, J.P. and Koppelman, F.S. (2002) A model of joint activity participation between household members, Transportation, 29(1), 49-72.

Goeree, J.K. and Holt, C.A. (2004) A theory of noisy introspection, Games and Economic Behavior, 46(2), 365-382.

Golob, T.F. and McNally, M.G. (1997) A model of activity participation and travel interactions between household heads, Transportation Research Part B: Methodological, 31(3), 177-194.

Guy, C.M. (1987) Recent advances in spatial interaction modelling: an application to the forecasting of shopping travel, Environment and Planning A, 19(2), 173-186.

Han, Q., Dellaert, B.G.C. Raaij, W.F. van and Timmermans, H.J.P. (2005) Integrating prospect theory and Stackelberg games to model strategic dyad behaviour of information providers and travellers: theory and numerical simulations, Transportation Research Record, forthcoming.

Harsanyi, J.C. (1967, 1968a, 1968b), Games with incomplete information played by "Bayesian” players, Management Science, 14, 159-182, 320-334, 486-502.

Hato, E., Taniguchi, M., Sugie, Y., Kuwahara, M. and Morita, H. (1999) Incorporating an information acquisition process into a route choice model with multiple information sources, Transportation Research Part C: Emerging Technologies, 7, 109-129.

Joh, C.H., Arentze, T.A. and Timmermans, H.J.P. (2002) Modelling individuals' activitytravel rescheduling heuristics: theory and numerical experiments, Transportation Research Record, 1807, 16-25.

Kahneman, D. and Tversky, A. (1982) The psychology of preferences, Scientific American, 246, 160-173.

Kamakura, W.A. and Srivastava, R.K. (1984) Predicting choice shares under conditions of brand interdependence, Journal of Marketing Research, 21, 420-432.

Kannan, P.K. and Kopalle, P.K. (2001) Dynamic pricing on the internet: importance and implications for consumer behaviour, International Journal of Electronic Commerce, 5(3), 63-83.

Kemperman, A.D.A.M, Borgers, A.W.J. and Timmermans, H.J.P. (2002) A semiparametric hazard model of activity timing and sequencing decisions during visits to theme parks using experimental design data, Tourism Analysis, 7(1), 1-14.

Kenyon, S. and Lyons, G. (2003) The value of integrated multimodal traveller information and its potential contribution to modal change, Transportation Research Part F: Traffic Psychology and Behaviour, 6(1), 1-21.

Khattak, A.J., Schofer, J.L. and Koppelman, F.S. (1993) Commuters' en-route diversion and return decisions: analysis and implications for advanced traveller information 
systems, Transportation Research Part A: Policy and Practice, 27(2), 101-111.

Kitamura, R. (1984) A model for daily time allocation to discretionary out-of-home activities and trips, Transportation Research Part B: Methodological, 18(3), 225266.

Kotler, P. (2000) Marketing Management, Prentice-Hall, NJ, USA.

Kozak, M. (2003) Measuring tourists' satisfaction with multiple destination attributes, Tourism Analysis, 7(3-4), 229-240.

Lee, H.-C. (2003) Modelling boater's choices among boating destinations in Michigan's Great Lakes, Tourism Analysis, 7(3-4), 217-228.

Louviere, J.J. and Timmermans, H.J.P. (1999) Using hierarchical information integration to model consumer response to possible planning actions: recreation destination choice illustration, Environment and Planning A, 22(3), 291-308.

Lu, X. and Pas, E.I. (1997) A structural equations model of the relationships among socio-demographics, activity participation and travel behaviour, Paper presented at the $76^{\text {th }}$ Annual Meeting of the Transportation Research Board, Washington, D.C., USA.

Lu, X. and Pas, E.I. (1999) Socio-demographics, activity participation and travel behaviour, Transportation Research Part A: Policy and Practice, 33(1), 1-18.

Luce, R. (1959) Individual Choice Behaviour: A Theoretical Analysis, Wiley, New York, USA.

Ma, J. and Goulias, K.G. (1997) A dynamic analysis of person and household activity and travel patterns using data from the first two waves in the Puget Sound Transportation panel, Transportation, 24(3), 309-331.

Mahmassani, H.S. and Jayakrishnan, R. (1991) System performance and user response under real-time information in a congested traffic corridor, Transportation Research Part A: Policy and Practice, 25(5), 293-307.

Mannering, F., Kim, S.G., Barfield, W. and Ng, L. (1994), Statistical analysis of commuters' route, mode, and departure time flexibility, Transportation Research Part C: Emerging Technologies, 2(1), 35-48.

Mansfield, Y. (1995) The value stretch model and its implications in detecting tourists, Journal of Travel and Tourist Marketing, 4, 71-92.

Manski, C. (1973) The Analysis of Qualitative Choice, Ph.D. Dissertation, Massachusetts Institute of Technology, Cambridge, MA, USA.

McFadden, D. (1976) The mathematical theory of demand models, in: Stopher, P. and Meyburg, A. (eds.) Behavioural Travel-Demand Models, Lexington Books, Lexington, MA, USA, 305-314.

McFadden, D. (1981) Econometric models of probabilistic choice, in: Manski, C. and McFadden, D. (eds.) Structural Analysis of Discrete Data, MIT Press, Cambridge, MA, USA, 198-272.

McFadden, D. (2001) Economic choices, American Economic Review, 91(3), 351-378.

McKelvey, R.D. and Palfrey T.R. (1995) Quantal response equilibria for normal form games, Games and Economic Behaviour, 10, 6-38. 
McKelvey, R.D. and Palfrey T.R. (1998) Quantal response equilibria for extensive form games, Experimental Economics, 1, 9-41.

Middelkoop, M. van, Borgers, A.W.J. Arentze, T.A. and Timmermans, H.J.P. (2001) Representing and predicting tourist choice behaviour, Tourism Analysis, 5(2), 113 118.

Moons, E., Wets, G., Vanhoof, K., Aerts, M., Arentze, T. and Timmermans, H. (2002) The impact of irrelevant attributes on the performance of classifier systems in generating activity schedules, Paper presented at the $81^{\text {st }}$ Annual Meeting of the Transportation Research Board, Washington, D.C., USA.

Morley, C.L. (1994), Discrete choice analysis of the impact of tourism prices, Journal of Travel Research, 33(2), 8-14.

Nyarko, Y. (2001) Learning, quantal response equilibrium and equilibrium in beliefs, (last accessed, Dec. 16 ${ }^{\text {th }} 2005$, http://www.nyarko.com/papers.htm).

Oppewal, H. (1995) Conjoint Experiment and Retail Planning, Ph.D. Dissertation, Eindhoven University of Technology, Eindhoven, The Netherlands.

Oppewal, H., Louviere, J.J. and Timmermans, H.J.P. (1994) Modelling hierarchical information integration processes with integrated conjoint choice experiments, Journal of Marketing Research, 31(1), 92-105.

Paddison, R. (1993) City marketing: image reconstruction and urban regeneration, Urban Studies, 30(2), 339-350.

Pendyala, R.M., Kitamura, R. and Reddy, D.V.G.P. (1998) Application of an activitybased travel demand model incorporating a rule-based algorithm, Environment and Planning B: Planning and Design, 25(5), 753-772.

Polak, J. (1987) A more general model of individual departure time choice, Proceedings of the PTRC Summer Annual Meeting, Seminar C, Transportation Planning Methods, 290, 247-258.

Polak, J. and Jones, P. (1993) The acquisition of pre-trip information: a stated preference approach, Transportation, 20, 179-198.

Polydoropoulou, A. and Ben-Akiva, M. (1998) The effect of advanced traveller information systems (ATIS) on travellers' behaviour, in: Emmerink, R.H.M. and Nijkamp, P. (eds.), Behavioural and Network Impacts of Driver Information Systems, Aldershot, Ashgate, UK, 317-352.

Prelec, D. (2000) Compound invariant weighting functions in prospect theory, in: Kahneman, D. and Tversky, A. (eds.) Choices, Values, and Frames, Cambridge University Press, Cambridge, UK, 67-92.

Ramarao, D. and Shugan, S.M. (1999) Strategic service pricing and yield management, Journal of Marketing, 63(1), 44-56.

Rasmusen, E. (1990) Games and Information: An Introduction to Game Theory, Basil Blackwell, Oxford and Cambridge, MA, USA.

Richards, M. and Ben-Akiva, M.E. (1974) A simultaneous destination and mode choice model for shopping trips, Transportation, 3, 343-356.

Rose, J. and Hensher, D.A. (2004) Modelling agent interdependency in group decision 
making, Transportation Research Part E: Logistics and Transportation Review, 40(1), 63-79.

Scott Bierman, H. and Fernandez, L. (1998) Game Theory with Economic Applications, Addison-Wesley, NY, USA.

Signorino, C. and Yilmaz, K. (2003) Strategic misspecification in regression models, Americn Journal of Political Science, 47(3), 551-566.

Signorino, C. (2003) Structure and uncertainty in discrete choice models, Political Analysis, 11(4), 316-344.

Srinivasan, K.K. and Mahmassani, H.S. (2000) Modelling inertia and compliance mechanisms in route choice behaviour under real-time information, Transportation Research Record, 1725, 45-53.

Stemerding, M.P., Oppewal, H. and Timmermans, H.J.P. (1999) A constraints-induced model of park choice, Leisure Sciences, 21(2), 145-158.

Swait, J.D. and Adamowicz, V. (2001) Choice environment, market complexity, and consumer behaviour: a theoretical and empirical approach for incorporating decision complexity into models of consumer choice, Organizational Behaviour and Human Decision Processes, 86(2), 141-167.

Timmermans, H.J.P. (1993) Retail environments and spatial shopping behaviour, in: Gärling, T. and Golledge, R.G. (eds.), Behaviour and Environment: Psychological and Geographical Approaches, Amsterdam, The Netherlands, 342-377.

Timmermans, H.J.P., Arentze, T.A. and Ettema, D.F. (2003) Learning and adaptation behaviour: empirical evidence and modelling issues, Proceedings ITS Conference, Eindhoven, The Netherlands.

Timmermans, H.J.P., Arentze, T.A. and Joh, C.H. (2002) Analyzing space-time behaviour: new approaches to old problems, Progress in Human Geography, 26(2), 175-190.

Von Neumann, J. and Morgenstern, O. (1944) Theory of Games and Economic Behaviour, Princeton University Press, Princeton, NJ, USA.

Wang, D. and Timmermans, H.J.P. (2000) Conjoint-based model of activity engagement, timing, scheduling and stop pattern formation, Transportation Research Record, 1718, 10-17.

Wilson, A.G. (1988) Store and shopping-centre location and size: a review of British research and practice, in: Wrigley, N. (ed.), Store Location, Store Choice and Market Analysis, Routledge, London, UK, 160-186.

Yim, Y. and Khattak, A.J. (2002) Traveller response to new dynamic information sources: analyzing corridor and area-wide behavioural surveys, Paper presented at the $81^{\text {st }}$ Annual Meeting of the Transportation Research Board, Washington, D.C., USA.

Zhang, J., Timmermans, H.J.P. and Borgers, A.W.J. (2002) Utility-maximizing model of household time use for independent, shared, and allocated activities incorporating group decision mechanisms, Transportation Research Record, 1807, 1-8. 


\section{Appendix A-1: $\quad$ Announcement of timing choice experiment}

Number of research experiment (administration): 167

Date: May 7, 2003

Name of supervisor: Prof dr W.F. van Raaij, P 811, tel: 2434

Name of researcher: Qi Han, SOBU-AIO, UvT-TUE

Description of research: Choice-experiment with a paper and pencil questionnaire

Date of experiment: May 22, 2003

Duration of the experiment (course credit): 1 hour ( 1 credit)

Total number of respondents required: 120

Location of the experiment: Room SZ-21

There are three possible participation times:

$2.30 \mathrm{pm}$

$4.00 \mathrm{pm}$

$5.30 \mathrm{pm}$

There are no restrictions on the number of participants for a participation time slot. However, depending on the number of students that register, there may be some delay and you may have to wait.

Registration by providing student name and ID number via e-mail:

tourisme-experiment@uvt.nl

Deadline of registration is May 20, 2003. 
Appendix A-2: $\quad$ Questionnaire of timing choice experiment

ID number:

Arriving time:

Gender:

Age:

\section{Part 1-A: Dinner timing choice (Non-strategic)}

In this section of the study, you'll be asked some questions about a possible dinner in different restaurants in London. Please imagine that you are in London for a weekend with a friend and that you're planning on going out for dinner on Saturday night.

From the travel guide you have selected a number of restaurants, none of which you have visited before. These restaurants all seem comparable in quality, ambience and price.

Imagine that on this particular evening you do not have any other fixed obligations. You will be offered a number of choices in which you are asked to select the restaurant where you prefer to make a reservation.

There are three fixed reservation times at each restaurant: 5:00 pm, 7:30 pm and 10:00 pm. You will be making a reservation in the restaurant of your choice.

In each choice scenario, you will also be told you may have to wait at the restaurant before you can get your dinner.

The reason for the wait is that although the restaurants allow you to book a dinner time, your specific table may not be available immediately. This waiting time is driven by random variation in the time the guests at your table take to finish their dinner.

The restaurants can however tell you the expected waiting time. They also provide an indication of the uncertainty of this wait. Once again, note that all restaurants are comparable in quality, ambience and price. Also note that the restaurants will tend to be crowded on a Saturday night. 
For each choice set please choose the dinner option that you prefer:

Situation 1

\begin{tabular}{|c|c|c|c|c|}
\hline & Restaurant 1 & Restaurant 2 & Restaurant 3 & None of the these \\
\hline Arrival time & $7: 30 \mathrm{pm}$ & $7: 30 \mathrm{pm}$ & $5: 00 \mathrm{pm}$ & \\
\hline Expected wait & 10 minutes & 0 minutes & 0 minutes & \\
\cline { 1 - 4 } $\begin{array}{c}\text { Probability of further } \\
10 \text { minutes wait }\end{array}$ & $40 \%$ & $40 \%$ & $20 \%$ & \\
\hline Your choice & & & & \\
\hline
\end{tabular}

Situation 2

\begin{tabular}{|l|c|c|c|c|}
\hline & Restaurant 1 & Restaurant 2 & Restaurant 3 & None of the these \\
\hline Arrival time & $5: 00 \mathrm{pm}$ & $10: 00 \mathrm{pm}$ & $10: 00 \mathrm{pm}$ & \\
\cline { 1 - 4 } Expected wait & 20 minutes & 20 minutes & 20 minutes & \\
\cline { 1 - 4 } $\begin{array}{l}\text { Probability of further } \\
10 \text { minutes wait }\end{array}$ & $40 \%$ & $40 \%$ & $20 \%$ & \\
\cline { 1 - 3 } Your choice & & & & \\
\hline
\end{tabular}

Situation 3

\begin{tabular}{|c|c|c|c|c|}
\hline & Restaurant 1 & Restaurant 2 & Restaurant 3 & None of the these \\
\hline Arrival time & $5: 00 \mathrm{pm}$ & $5: 00 \mathrm{pm}$ & $7: 30 \mathrm{pm}$ & \\
\hline Expected wait & 10 minutes & 10 minutes & 10 minutes & \\
\cline { 1 - 3 } $\begin{array}{c}\text { Probability of further } \\
10 \text { minutes wait }\end{array}$ & $0 \%$ & $20 \%$ & $0 \%$ & \\
\hline Your choice & & & & \\
\hline
\end{tabular}

Situation 4

\begin{tabular}{|c|c|c|c|c|}
\hline & Restaurant 1 & Restaurant 2 & Restaurant 3 & None of the these \\
\hline Arrival time & $7: 30 \mathrm{pm}$ & $7: 30 \mathrm{pm}$ & $5: 00 \mathrm{pm}$ & \\
\cline { 1 - 4 } Expected wait & 0 minutes & 10 minutes & 20 minutes & \\
\cline { 1 - 3 } $\begin{array}{c}\text { Probability of further } \\
10 \text { minutes wait }\end{array}$ & $20 \%$ & $20 \%$ & $0 \%$ & \\
\hline Your choice & & & & \\
\hline
\end{tabular}

Situation 5

\begin{tabular}{|c|c|c|c|c|}
\hline & Restaurant 1 & Restaurant 2 & Restaurant 3 & None of the these \\
\hline Arrival time & $10: 00 \mathrm{pm}$ & $7: 30 \mathrm{pm}$ & $10: 00 \mathrm{pm}$ & \\
\hline Expected wait & 0 minutes & 20 minutes & 0 minutes & \\
\cline { 1 - 3 } $\begin{array}{c}\text { Probability of further } \\
10 \text { minutes wait }\end{array}$ & $0 \%$ & $0 \%$ & $20 \%$ & \\
\hline Your choice & & & & \\
\hline
\end{tabular}




\section{Situation 6}

\begin{tabular}{|c|c|c|c|c|}
\hline & Restaurant 1 & Restaurant 2 & Restaurant 3 & None of the these \\
\hline Arrival time & $10: 00 \mathrm{pm}$ & $5: 00 \mathrm{pm}$ & $10: 00 \mathrm{pm}$ & \\
\hline Expected wait & 20 minutes & 0 minutes & 10 minutes & \\
\cline { 1 - 3 } $\begin{array}{c}\text { Probability of further } \\
10 \text { minutes wait }\end{array}$ & $0 \%$ & $40 \%$ & $20 \%$ & \\
\hline Your choice & & & & \\
\hline
\end{tabular}

Situation 7

\begin{tabular}{|c|c|c|c|c|}
\hline & Restaurant 1 & Restaurant 2 & Restaurant 3 & None of the these \\
\hline Arrival time & $5: 00 \mathrm{pm}$ & $5: 00 \mathrm{pm}$ & $7: 30 \mathrm{pm}$ & \\
\cline { 1 - 4 } Expected wait & 10 minutes & 20 minutes & 0 minutes & \\
\cline { 1 - 3 } $\begin{array}{c}\text { Probability of further } \\
10 \text { minutes wait }\end{array}$ & $40 \%$ & $20 \%$ & $0 \%$ & \\
\hline Your choice & & & & \\
\hline
\end{tabular}

Situation 8

\begin{tabular}{|c|c|c|c|c|}
\hline & Restaurant 1 & Restaurant 2 & Restaurant 3 & None of the these \\
\hline Arrival time & $10: 00 \mathrm{pm}$ & $10: 00 \mathrm{pm}$ & $10: 00 \mathrm{pm}$ & \multirow{2}{*}{10 minutes } \\
\cline { 1 - 4 } Expected wait & 0 minutes & 10 minutes & \multirow{2}{*}{$0 \%$} & \\
\hline $\begin{array}{c}\text { Probability of further } \\
10 \text { minutes wait }\end{array}$ & $40 \%$ & $40 \%$ & & \\
\hline Your choice & & & & \\
\hline
\end{tabular}

Situation 9

\begin{tabular}{|c|c|c|c|c|}
\hline & Restaurant 1 & Restaurant 2 & Restaurant 3 & None of the these \\
\hline Arrival time & $5: 00 \mathrm{pm}$ & $7: 30 \mathrm{pm}$ & $7: 30 \mathrm{pm}$ & \\
\hline Expected wait & 0 minutes & 20 minutes & 20 minutes & \\
\cline { 1 - 3 } $\begin{array}{c}\text { Probability of further } \\
10 \text { minutes wait }\end{array}$ & $0 \%$ & $20 \%$ & $40 \%$ & \\
\hline Your choice & & & & \\
\hline
\end{tabular}




\section{Part 1-B: Dinner timing choice (Strategic)}

In this section of the study, you'll be asked some questions about a possible dinner in different restaurants in London. Please imagine that you are in London for a weekend with a friend and that you're planning on going out for dinner on Saturday night.

From the travel guide, you have selected a number of restaurants none of which you have visited before. These restaurants all seem comparable in quality, ambience and price.

Imagine that on this particular evening you do not have any other fixed obligations. You will be offered a number of choices in which you are asked to select the restaurant at where you prefer to make a reservation.

There are three fixed reservation times at each restaurant: 5:00 pm, 7:30 pm and 10:00 $\mathrm{pm}$. You will be making a reservation in the restaurant of your choice.

In each choice scenario, you will also be told you may have to wait at the restaurant before you can get your dinner. The reason for the wait is that although the restaurants allow you to book a dinner time, they assign guests arriving at each time on a first come first serve basis. Thus, depending on the number of guests, each guest on average will have to wait longer for a table. The length of this waiting time is not known before you get to the restaurant.

However, the travel guide provides you with some indication of the booking policy in each restaurant. This may help you in deciding if you should wish to make an early or a later booking. Once again, note that all restaurants are comparable in quality, ambience and price. Also note that restaurants tend to be crowded on a Saturday night.

For each choice set, please choose the dinner option that you prefer:

Situation 1

\begin{tabular}{|c|c|c|c|c|}
\hline & Restaurant 1 & Restaurant 2 & Restaurant 3 & None of the these \\
\hline Arrival time & $5: 00 \mathrm{pm}$ & $5: 00 \mathrm{pm}$ & $7: 30 \mathrm{pm}$ & \\
\cline { 1 - 4 } $\begin{array}{c}\text { Number of } \\
\text { guests relative to } \\
\text { capacity }\end{array}$ & More than capacity & Close to capacity & At full capacity & \\
\hline Your choice & & & & \\
\hline
\end{tabular}

Situation 2

\begin{tabular}{|c|c|c|c|c|}
\hline & Restaurant 1 & Restaurant 2 & Restaurant 3 & None of the these \\
\hline Arrival time & $10: 00 \mathrm{pm}$ & $5: 00 \mathrm{pm}$ & $10: 00 \mathrm{pm}$ & \\
\cline { 1 - 4 } $\begin{array}{c}\text { Number of } \\
\text { guests relative to } \\
\text { capacity }\end{array}$ & Close to capacity & At full capacity & At full capacity & \\
\hline Your choice & & & & \\
\hline
\end{tabular}


Situation 3

\begin{tabular}{|c|c|c|c|c|}
\hline & Restaurant 1 & Restaurant 2 & Restaurant 3 & None of the these \\
\hline Arrival time & $7: 30 \mathrm{pm}$ & $7: 30 \mathrm{pm}$ & $10: 00 \mathrm{pm}$ & \\
\cline { 1 - 4 } $\begin{array}{c}\text { Number of } \\
\text { guests relative to } \\
\text { capacity }\end{array}$ & Close to capacity & More than capacity & More than capacity & \\
\cline { 1 - 3 } Your choice & & & & \\
\hline
\end{tabular}

Please answer the following question:

When making your choices, you were asked to choose a dinner time

a) How long did you expect you would have to wait before you could have a seat for dinner?

If you would come at 5:00 pm and close to capacity

...minutes

If you would come at 5:00 pm and at full capacity

...minutes

If you would come at 5:00 pm and more than capacity

...minutes

If you would come at 7:30 pm and close to capacity

...minutes

If you would come at 7:30 pm and at full capacity

...minutes

If you would come at 7:30 pm and more than capacity

...minutes

If you would come at 10:00 pm and close to capacity

...minutes

If you would come at 10:00 pm and at full capacity

...minutes

If you would come at 10:00 pm and more than capacity

...minutes

b) What did you think was the probability that you would have to wait 10 minutes more than you anticipated?

If you would come at 5:00 pm and close to capacity

...minutes

If you would come at 5:00 pm and at full capacity

...minutes

If you would come at 5:00 pm and more than capacity

...minutes

If you would come at 7:30 pm and close to capacity

...minutes

If you would come at 7:30 pm and at full capacity

...minutes

If you would come at 7:30 pm and more than capacity

...minutes

If you would come at 10:00 pm and close to capacity

...minutes

If you would come at 10:00 pm and at full capacity

...minutes

If you would come at 10:00 pm and more than capacity

...minutes 


\section{Part 2-A: Participation timing choice (Real world)}

In this section, you are asked to answer the following questions:

When registering for this experiment, you were asked to choose a start time

a) How long did you expect you would have to wait before you could start the experiment?

If you would come at 2:30 pm

...minutes

If you would come at 4:00 pm

...minutes

If you would come at 5:30 pm

...minutes

b) What did you think was the probability that you would have to wait 10 minutes more than you anticipated?

If you would come at $2: 30 \mathrm{pm} \quad \ldots \%$

If you would come at $4: 00 \mathrm{pm} \quad \ldots \%$

If you would come at $5: 30 \mathrm{pm} \quad \ldots \%$

c) How many other students did you think would register?
At $\quad 2.30 \mathrm{pm}$
...students
At $\quad 4.00 \mathrm{pm}$
...students
At $\quad 5.30 \mathrm{pm}$
...students

d) How long did you expect that this experiment would take you to complete (not including waiting)? ... minutes

e) How long did you expect that this experiment would take the average person to complete (not including waiting)? ... minutes 


\section{Part 2-B: $\quad$ Participation timing choice (Non-strategic)}

We now would like to learn more about your preferences for different start times for experiments in general. You will be offered a number of choices in which you are asked to select the start time option that you prefer. Please imagine that on the day of the experiment you do not have any other obligations. Also imagine that you may have to wait some time before you can start with the experiment. The reason for the wait is that the experiment requires an elaborate computer and laboratory set-up. Note that you don't have to wait for others to complete the experiment.

For each choice set, please choose the start time that you prefer:

Situation 1

\begin{tabular}{|c|c|c|c|c|}
\hline & Experiment 1 & Experiment 2 & Experiment 3 & None of the these \\
\hline Start time & $2: 30 \mathrm{pm}$ & $2: 30 \mathrm{pm}$ & $5: 30 \mathrm{pm}$ & \multirow{2}{*}{10 minutes } \\
\cline { 1 - 4 } Expected wait & 20 minutes & 10 minutes & \multirow{2}{*}{$0 \%$} & \\
\hline $\begin{array}{c}\text { Probability of } 10 \text { minutes } \\
\text { additional delay }\end{array}$ & $0 \%$ & $20 \%$ & & \\
\hline Your choice & & & & \\
\hline
\end{tabular}

Situation 2

\begin{tabular}{|c|c|c|c|c|}
\hline & Experiment 1 & Experiment 2 & Experiment 3 & None of the these \\
\hline Start time & $5: 30 \mathrm{pm}$ & $2: 30 \mathrm{pm}$ & $4: 00 \mathrm{pm}$ & \\
\cline { 1 - 4 } Expected wait & 20 minutes & 0 minutes & 20 minutes & \\
\cline { 1 - 3 } $\begin{array}{c}\text { Probability of } 10 \text { minutes } \\
\text { additional delay }\end{array}$ & $40 \%$ & $20 \%$ & $20 \%$ & \\
\hline Your choice & & & & \\
\hline
\end{tabular}

Situation 3

\begin{tabular}{|c|c|c|c|c|}
\hline & Experiment 1 & Experiment 2 & Experiment 3 & None of the these \\
\hline Start time & $5: 30 \mathrm{pm}$ & $2: 30 \mathrm{pm}$ & $4: 00 \mathrm{pm}$ & \\
\cline { 1 - 4 } Expected wait & 10 minutes & 0 minutes & 0 minutes & \\
\cline { 1 - 3 } $\begin{array}{c}\text { Probability of } 10 \text { minutes } \\
\text { additional delay }\end{array}$ & $20 \%$ & $0 \%$ & $0 \%$ & \\
\hline Your choice & & & & \\
\hline
\end{tabular}

Situation 4

\begin{tabular}{|c|c|c|c|c|}
\hline & Experiment 1 & Experiment 2 & Experiment 3 & None of the these \\
\hline Start time & $5: 30 \mathrm{pm}$ & $5: 30 \mathrm{pm}$ & $5: 30 \mathrm{pm}$ & \\
\cline { 1 - 4 } Expected wait & 0 minutes & 10 minutes & 20 minutes & \\
\cline { 1 - 3 } $\begin{array}{c}\text { Probability of } 10 \text { minutes } \\
\text { additional delay }\end{array}$ & $20 \%$ & $40 \%$ & $0 \%$ & \\
\hline Your choice & & & & \\
\hline
\end{tabular}




\section{Situation 5}

\begin{tabular}{|c|c|c|c|c|}
\hline & Experiment 1 & Experiment 2 & Experiment 3 & None of the these \\
\hline Start time & $2: 30 \mathrm{pm}$ & $2: 30 \mathrm{pm}$ & $5: 30 \mathrm{pm}$ & \\
\hline Expected wait & 20 minutes & 20 minutes & 0 minutes & \\
\hline $\begin{array}{c}\text { Probability of 10 minutes } \\
\text { additional delay }\end{array}$ & $20 \%$ & $40 \%$ & $40 \%$ & \\
\hline Your choice & & & & \\
\hline
\end{tabular}

Situation 6

\begin{tabular}{|c|c|c|c|c|}
\hline & Experiment 1 & Experiment 2 & Experiment 3 & None of the these \\
\hline Start time & $2: 30 \mathrm{pm}$ & $2: 30 \mathrm{pm}$ & $4: 00 \mathrm{pm}$ & \\
\cline { 1 - 4 } Expected wait & 0 minutes & 10 minutes & 20 minutes & \\
\cline { 1 - 3 } $\begin{array}{c}\text { Probability of } 10 \text { minutes } \\
\text { additional delay }\end{array}$ & $40 \%$ & $40 \%$ & $40 \%$ & \\
\hline Your choice & & & & \\
\hline
\end{tabular}

Situation 7

\begin{tabular}{|c|c|c|c|c|}
\hline & Experiment 1 & Experiment 2 & Experiment 3 & None of the these \\
\hline Start time & $5: 30 \mathrm{pm}$ & $4: 00 \mathrm{pm}$ & $2: 30 \mathrm{pm}$ & \multirow{2}{*}{10 minutes } \\
\cline { 1 - 4 } Expected wait & 0 minutes & 20 minutes & \multirow{2}{*}{$0 \%$} & \\
\cline { 1 - 4 } $\begin{array}{c}\text { Probability of } 10 \text { minutes } \\
\text { additional delay }\end{array}$ & $0 \%$ & $0 \%$ & & \\
\cline { 1 - 4 } Your choice & & & & \\
\hline
\end{tabular}

Situation 8

\begin{tabular}{|c|c|c|c|c|}
\hline & Experiment 1 & Experiment 2 & Experiment 3 & None of the these \\
\hline Start time & $4: 00 \mathrm{pm}$ & $4: 00 \mathrm{pm}$ & $4: 00 \mathrm{pm}$ & \\
\cline { 1 - 4 } Expected wait & 0 minutes & 10 minutes & 0 minutes & \\
\cline { 1 - 3 } $\begin{array}{c}\text { Probability of } 10 \text { minutes } \\
\text { additional delay }\end{array}$ & $20 \%$ & $40 \%$ & $40 \%$ & \\
\hline Your choice & & & & \\
\hline
\end{tabular}

Situation 9

\begin{tabular}{|c|c|c|c|c|}
\hline & Experiment 1 & Experiment 2 & Experiment 3 & None of the these \\
\hline Start time & $4: 00 \mathrm{pm}$ & $4: 00 \mathrm{pm}$ & $5: 30 \mathrm{pm}$ & \multirow{2}{*}{20 minutes } \\
\cline { 1 - 4 } Expected wait & 10 minutes & 10 minutes & \multirow{2}{*}{$20 \%$} & \\
\cline { 1 - 3 } $\begin{array}{c}\text { Probability of } 10 \text { minutes } \\
\text { additional delay }\end{array}$ & $0 \%$ & $20 \%$ & & \\
\cline { 1 - 3 } Your choice & & & & \\
\hline
\end{tabular}




\section{Part 2-C: Participation timing choice (Strategic)}

Finally, we would like to ask your preferences for participating in a follow-up experiment. This experiment will take place in the near future, most probably on Wednesday in two weeks time. In contrast to the current experiment, the next experiment will be run in the computer lab. Therefore, the number of students who can participate at the same time is limited and you may have to wait before you can participate.

This situation occurs if the number of participants at any given time is greater than 25 . However, based on the preferences for the current experiment, we can also give you an indication of the popularity of the different times. In total we will need 100 people for the experiment. We will stop recruiting participants if we have a total of 100 participants.

The experiment will be run in 3 sessions (2:30 pm, 4:00 pm and 5:30 pm), and you can freely choose one of these times (keeping in mind you may have to wait). Please indicate at which time you would like to participate in this follow-up experiment.

\begin{tabular}{|c|c|c|c|c|}
\hline Information set 1 & Option 1 & Option 2 & Option 3 & None of the these \\
\hline Start time & $2: 30 \mathrm{pm}$ & $4: 00 \mathrm{pm}$ & $5: 30 \mathrm{pm}$ & \multirow{2}{*}{} \\
\cline { 1 - 4 } $\begin{array}{c}\text { Percentage of students } \\
\text { preferring this time }\end{array}$ & $17 \%$ & $33 \%$ & $50 \%$ & \\
\hline Your choice & & & & \\
\hline
\end{tabular}

Please answer the following questions:

a) How many students do you think would actually register at each time?
At $\quad 2.30 \mathrm{pm}$
...students
At $\quad 4.00 \mathrm{pm}$
...students
At $\quad 5.30 \mathrm{pm}$
...students

b) How long do you think the next experiment would take you to complete (not including waiting)? ... minutes

c) How long do you think the next experiment would take the average person to complete (not including waiting)? ... minutes

d) How long would you expect you would have to wait?

If you would come at 2:30 pm

...minutes

If you would come at 4:00 pm

...minutes

If you would come at 5:30 pm

...minutes

e) What do you think is the probability that you would have to wait 10 minutes more than you anticipated?

If you would come at $2: 30 \mathrm{pm}$

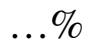

If you would come at 4:00 pm

$\ldots \%$

If you would come at 5:30 pm

$\ldots \%$ 


\section{Appendix B-1: $\quad$ Announcement of repeated choice experiment}

\begin{tabular}{|c|}
\hline Number of research experiment (administration): 335 \\
\hline Date: March 15, 2005 \\
\hline Name of supervisor: Prof dr W.F. van Raaij, P 811, tel: 2434 \\
\hline Name of researcher: Qi Han, SOBU-AIO, UvT-TUE \\
\hline Description of research: Choice-experiment with strategic anticipation \\
\hline Date of experiment: April 12-15, 2005 \\
\hline $\begin{array}{l}\text { Duration of the experiment (course credit): } 1 \text { hour }(1 \text { credit). Based on the } \\
\text { performance one out of ten participants will win } € 10 \text {. }\end{array}$ \\
\hline Total number of respondents required: 100 \\
\hline Location of the experiment: ESP Laboratory \\
\hline $\begin{array}{l}\text { There are six possible participation times each day: } \\
10.00 \mathrm{am} \\
11.00 \mathrm{am} \\
12.00 \mathrm{am} \\
1.00 \mathrm{pm} \\
2.00 \mathrm{pm} \\
3.00 \mathrm{pm} \\
\text { There is a maximum of } 12 \text { participants on each of these times. }\end{array}$ \\
\hline $\begin{array}{l}\text { Registration by providing student name and ID number via signing up on the attached } \\
\text { form. } \\
\text { Deadline of registration is April 10, } 2005 \text {. }\end{array}$ \\
\hline
\end{tabular}


Appendix B-2: Overview of the tasks

\begin{tabular}{|c|c|c|c|c|c|}
\hline $\begin{array}{c}\text { Average } \\
\text { performance }\end{array}$ & Task & $\begin{array}{c}\text { Recommendation } \\
\text { objective }\end{array}$ & $\begin{array}{l}\text { Preference } \\
\text { certainty }\end{array}$ & $\begin{array}{l}\text { Compliance } \\
\text { rate }(q)\end{array}$ & $\begin{array}{l}\text { Marginal } \\
\text { disutility }\end{array}$ \\
\hline 0.92 & 1 & no recommendation & uncertain & $0 \%$ & 0.05 \\
\hline 0.97 & 2 & no recommendation & certain & $0 \%$ & 0.05 \\
\hline 0.88 & 3 & no recommendation & uncertain & $0 \%$ & 0.1 \\
\hline 0.94 & 4 & no recommendation & certain & $0 \%$ & 0.1 \\
\hline 0.89 & 5 & equal usage & uncertain & $15 \%$ & 0.1 \\
\hline 0.95 & 6 & equal usage & certain & $5 \%$ & 0.1 \\
\hline 0.87 & 7 & equal usage & uncertain & $5 \%$ & 0.05 \\
\hline 0.95 & 8 & equal usage & certain & $15 \%$ & 0.05 \\
\hline 0.89 & 9 & welfare maximum & uncertain & $25 \%$ & 0.1 \\
\hline 0.93 & 10 & welfare maximum & certain & $15 \%$ & 0.1 \\
\hline 0.92 & 11 & welfare maximum & uncertain & $15 \%$ & 0.05 \\
\hline 0.94 & 12 & welfare maximum & certain & $25 \%$ & 0.05 \\
\hline 0.93 & 13 & user equity & uncertain & $35 \%$ & 0.1 \\
\hline 0.96 & 14 & user equity & certain & $25 \%$ & 0.1 \\
\hline 0.94 & 15 & user equity & uncertain & $25 \%$ & 0.05 \\
\hline 0.96 & 16 & user equity & certain & $35 \%$ & 0.05 \\
\hline 0.94 & 17 & equal usage & uncertain & $25 \%$ & 0.1 \\
\hline 0.97 & 18 & equal usage & certain & $25 \%$ & 0.05 \\
\hline 0.92 & 19 & user equity & uncertain & $15 \%$ & 0.05 \\
\hline 0.96 & 20 & user equity & certain & $15 \%$ & 0.1 \\
\hline 0.93 & 21 & equal usage & uncertain & $5 \%$ & 0.1 \\
\hline 0.96 & 22 & equal usage & certain & $5 \%$ & 0.05 \\
\hline 0.93 & 23 & equal usage & uncertain & $15 \%$ & 0.05 \\
\hline 0.96 & 24 & equal usage & certain & $15 \%$ & 0.1 \\
\hline 0.94 & 25 & welfare maximum & uncertain & $15 \%$ & 0.1 \\
\hline 0.96 & 26 & welfare maximum & certain & $15 \%$ & 0.05 \\
\hline 0.94 & 27 & welfare maximum & uncertain & $25 \%$ & 0.05 \\
\hline 0.97 & 28 & welfare maximum & certain & $25 \%$ & 0.1 \\
\hline 0.95 & 29 & user equity & uncertain & $25 \%$ & 0.1 \\
\hline 0.97 & 30 & user equity & certain & $25 \%$ & 0.05 \\
\hline 0.96 & 31 & user equity & uncertain & $35 \%$ & 0.05 \\
\hline 0.98 & 32 & user equity & certain & $35 \%$ & 0.1 \\
\hline
\end{tabular}




\section{Appendix B-3: $\quad$ Screen shot of the instruction}

Introduction

Thank you for participating in this experiment. The objective of this study is to get insight into how people make decisions when they have to take into account other people's decision-making and how they improve their performance through learning by repeated interaction. Your task in the experiment is to make smart choices to earn as many points as possible. If you read the instructions carefully and make good decisions you can earn a considerable amount of money. At end of the experiment, the scores of all participants are compared, if you are the best of the 10 person group, you will get rewarded. The experiment will take about one hour. During the experiment, you are not allowed to speak or communicate with the other participants. If you have a question while the experiment is going on, please ask the instructor.

Each one of you will play against the computer that simulates another 100 players. The computer uses a behavioural model to mimic the behaviour of those players. Like your own choice, this model is based on the number of points attached to each option and the expected number of other players that will choose the option. The decisions that you and the simulated other 100 participants make will determine the amount that you will earn. That is to say, the amount depends on both your and the other players' decisions.

In the experiment, there will be 32 tasks in total. Within each task, there are 15 rounds. In each round you need to make a choice. At the beginning of each task, all of you will be informed about the default payoff for three options. That is the maximum number of points you could possibly get for choosing that option. You can take that as base-line points. Then, you and 100 simulated players make a choice simultaneously. Each of you receives the option default payoff minus an amount that depends on how many others choose the same option. With each additional person that chooses the same option as you, the amount you receive decreases with some fixed number of points. You have to learn this amount by playing the game. Your earning will be calculated in the following way.

Suppose that the option that you choose has a default payoff of 10 points. There are in total 60 players who choose the same option, and the number of points decreases by 0.1 with each additional person. Then,

Your payoff $=10-0.1^{*} 60=4$ (points)

In some tasks, the computer will give you recommended option. These recommendations are based on one of the three objectives: maximizing the total payoff for all participants, generating equal payoff for everybody or generating an equal distribution across the three options. The recommendation is the same for all of you. And it may or may not help you and the simulated players to earn more points. Because if all of you follow the recommendation, that option will be chosen by too many players, which will decrease the amount you can earn. So you need to figure out how others respond to receive the highest number of points.

Before you really start, you need to answer 3 questions to help checking whether you read this introduction careful enough. If you answered all the questions correctly, you will be offered some tips for the experiment. Then, you will have 2 trial tasks to get use to the play. During the whole experiment you can always review the tips.

\section{OK}




\section{Appendix B-4: $\quad$ Screen shot of the tips}

\section{Tips}

1. The option default payoff is the maximum of points you can earn for choosing that option, which is equal to the number of base-line points.

2. The points you receive for choosing the option will be calculated based on two parts: the default points attached to that option and the number of players choosing that option.

3. The recommended option may or may not help you. You need to find out how others react to it.

An example of how to make a choice:

Suppose that the option default points are $\{10,8,6\}$.

If you expect the number of players choosing each option is $\{60,25,15\}$.

Then your possible number of earned points for each option is:

option 1: $10-0.1 * 60=4.0$ (points)

option 2: $8-0.1 * 25=5.5$ (points)

option 3: $6-0.1 * 15=4.5$ (points)

So, your best choice would be option 2 .

But if you expect the number of players choosing each option is $\{45,40,15\}$.

Then your possible number of earned points will change to:

option 1: $10-0.1 * 45=5.5$ (points)

option 2: $8-0.1 * 40=4.0$ (points)

option 3: $6-0.1 * 15=4.5$ (points)

Now your best choice would be option 1 .

Please remember you need to figure out how others make their choice to increase the number of points that you will earn. 


\section{Appendix B-5: $\quad$ A screen shot after subjects made a choice}

\begin{tabular}{|c|c|c|}
\hline \multicolumn{2}{|l|}{ Experiment } & a回 \\
\hline \multicolumn{2}{|c|}{ Task $=7$} & \\
\hline \multicolumn{3}{|c|}{ Your base-line points } \\
\hline option 1 & option 2 & option 3 \\
\hline 2.2 & 5.6 & 6.0 \\
\hline \multicolumn{3}{|c|}{ RECOMMENDED } \\
\hline \multirow[t]{2}{*}{0} & 0 & 0 \\
\hline & \multicolumn{2}{|l|}{ OK } \\
\hline \multicolumn{3}{|c|}{ You chose option 3. Your score is $6.0-4.6$, because 46 others chose this option. } \\
\hline Last Earned Points: & Total Earned Points: & 191.3 \\
\hline \multicolumn{3}{|c|}{ Review tips... } \\
\hline
\end{tabular}




\section{Author index}

A

Adamowicz, 66

Adler, 11

Aerts, 9

Ajzen, 87

Alba, 10

Anderson, 30

Aoki, 12

Arentze, 9, 10, 11, 91, 127

Arnott, 11

Arthur, 43

Axhausen, 10

B

Bajari, 15

Barfield, 9, 11

Bargeman, 10
Basar, 42

Baxter, 6

Bayer, 15

Ben-Akiva, 5, 7, 9, 11, 30

Bhat, 9

Blue, 11

Blume, 12

Bierlaire, 9

Bolduc, 9

Borgers, 9, 10, 12

Borsch-Supan, 9

Bottom, 11

Bowman, 9

Brännäs, 9

Brewer, 15

Brock, 12 
C

Capra, 30

Chen, 10, 15

Chorus, 10

Crouch, 7

D

Dellaert, 1, 9, 126

Delquie, 9

Desiraju, 10

Dingus, 11

Dugundji, 12

Durlauf, 12

E

Emmerink, 10

Engemann, 49

Esarey, 34

Ettema, 9, 91

Ewing, 6

F

Fehr, 37

Fernandez, 14, 21, 41

Fischbacher, 37

Fleischmann, 10

Fotheringham, 6, 7

Fudenberg, 25, 42

G

Gärling, 9, 23

Gliebe, 12

Goeree, 30, 35, 36, 52, 54, 85, 108

Golledge, 9

Golob, 9
Gomez, 30

Gopal, 9

Gopinath, 9

Goulias, 9

Gulyas, 12

Guy, 6

H

Hall, 10

Han, 126

Harsanyi, 19

Hato, 11

Hensher, 15

Hofman, 12

Holm, 9

Holt, 30, 35, 36, 52, 54, 85, 108

J

Janiszewski, 16

Jayakrishnan, 11

Joh, 9, 10

Jones, 11

K

Kahn, 15

Kalen, 23

Kamakura, 9

Kannan, 10

Kaysi, 11

Kemperman, 10

Kenyon, 11

Khattak, 11

Kim, 9

Kitamura, 9

Kopalle, 10 
Koppelman, 9, 11, 12

Kotler, 1

Kozak, 10

Kuwahara, 11

$\mathbf{L}$

Larichey, 9

Lee, 10

Lerman, 5, 7, 30

Lindberg, 9

Lindsey, 11

Louviere, 7, 9

Lu, 9

Luce, 27, 30

Lutz, 16

Lynch, 16

Lyons, 11

M

Ma, 9

Mahmassani, 10, 11, 43

Mannering, 9

Mansfield, 9

Manski, 24

McFadden, 9, 27, 30

McKelvey, 27, 29, 34, 35, 55, 85, 108

McMillan, 15

McNally, 9

Middelkoop, 10

Molin, 10

Moons, 9

Morikawa, 9

Morita, 11

Morley, 9
$\mathbf{N}$

$\mathrm{Ng}, 9$

Nijkamp, 10

Nyarko, 29

O

Olsder, 42

Oppewal, 6, 9, 10

$\mathbf{P}$

Paddison, 1

Palfrey, 27, 29, 34, 35, 55, 85, 108

Palma, 11

Pas, 9

Pendyala, 9

Polak, 9, 11

Polydoropoulou, 9, 11

Pyke, 10

$\mathbf{R}$

Ramming, 11

Radtke, 40

Raaij, 140

Rao, 9

Rasmusen, 14, 26

Reddy, 9

Richards, 7

Rietveld, 10

Romanus, 23

Rose, 15

Rueben, 15

S

Sachs, 40

Sawyer, 16 
Schmidt, 37

Schofer, 11

Scott Bierman, 14, 21, 41

Selart, 23

Shugan, 10

Signorino, 34

Srinivasan, 43

Srivastava, 9

Stemerding, 10

Stremersch, 1

Sugie, 11

Sun, 10

Swait, 66

$\mathbf{T}$

Taniguchi, 11

Timmermans, 6, 7, 9-12, 91, 126

Tirole, 25, 42

V

Vanhoof, 9

Vilhelmson, 23

Von Neumann-Morgenstern, 19

W

Walker, 9, 12

Wang, 9

Waerden, 9

Wee, 10

Weitz, 16

Wetz, 9

Wilson, 6

Wong, 7

Wood, 16
Y

Yim, 11

Z

Zhang, 12 


\section{Subject index}

A

activity-based, 9

aggregate, 3, 6, 7, 10, 11, 13, 15, 16, 18, $28,29,39,49,52,63,66,99,122$, 124

anticipation, 2-4, 11, 16, 17, 18, 24, 26, $32,37-39,43,66,67,69,70,75-$ 77, 84-88, 89-91, 93, 102, 106$108,110,113-117,119,122-124$

attribute, 7, 8, 11, 23

\section{B}

Bayesian Nash equilibrium, 19, 21, 22, $25,26,29,30,39$

C

choice alternative, 7-11, 13, 17, 22, 23, $50,52,56$ choice behaviour, 4, 6, 9, 11, 12, 14, 16$18,22,27,38,39,50,63,65,67$, $84,90,92,99,100,113,116,119$, $122,124,126$

choice probability, $8,9,13,24,26,28$ $31,34-36,43,47,49,58,95,122$, 126

common knowledge, 19, 22, 25, 26, 28, 33,40

compliance, 43, 44, 57, 60, 93-95, 99$101,103-106,112,118,123$

congestion, 2-4, 10, 11, 16, 17, 23, 37, $38,50,52,53,56,59,65-70,73-$ $75,77,81-88,89-95,101-103$, 106-119, 121-123, 126

conjecture, 25, 26, 43, 49, 56, 58, 60, 63, $67,84,122$

consistency, 11, 14, 24, 26, 28, 34, 111

constraint, 18, 26, 70, 121

contrast parameter, 80-82, 106 
D

data, 3, 4, 7-9, 13, 15, 33, 34, 37, 50, 63, $65,67,69-71,74,77,80-82,84$, $87,88,90,92,97,98,103,106-$ $108,110,111,113-119,123,124$

decision, 3, 5, 8, 10-15, 17, 21, 23, 24, 26-30, 32, 33, 35-39, 42, 46, 47, 50, 51, 57, 58, 66, 68, 72, 73, 75, $80,90,91,93,105,116,124-126$

decision support, 125

decision-making, 8, 13, 14, 24, 33, 37, $50,51,57,58,66,68,73,75,91$, 105

disaggregate, 19, 46, 63, 122, 124

discrete choice, $6,7,9,12,15-18,22-24$, $26,30,33,46,121,122$

dynamic, 6, 10, 15, 91, 127

E

effect, $2,10-13,16,23,32,33,50,52-$ 54, 56, 59, 60, 66, 69, 71-73, 75, $77,79,81,84,90,93,98-111,113-$ $119,122-124,126$

empirical game theory, 66, 91

endogenous, 13, 14, 16, 23, 24, 33, 34, $46,63,114,122,124,126$

equal usage, $10,41,42,44-46,56-62$, $94,99,100,122$

equity, 41, 45, 46, 56, 57, 59, 60, 62, 94, $99,100,122$

error, 7, 8, 20, 22, 24-29, 31-36, 38, 54, $57,60,66,72,75,91,92,100$, $107,108,113,116,118,123$

estimates, 33-35, 72-82, 84, 85, 90, 103 $106,108,109,111,112,115,118$

exogenous, 13, 14, 22, 24, 34, 46, 56, 63, $66,122,124,126$

expectation, $2,4,11,13,14,17,18,24$, $28,35,36,39,42-44,47,50,56$, 60, 63, 66, 77, 84-87, 90, 97, 101, $106,115,122,126,127$ expected utility, 11, 18, 19, 21, 25, 28, 35

experiment, 4, 8, 32, 65, 68, 69, 70, 72, 73, 75, 77, 83, 84, 87-89, 93, 95, $96,89,90,92-97,101,114,137$

\section{F}

face validity, 4, 47, 63, 65, 122

full-factorial, 68, 69, 70, 73, 77, 93

\section{G}

game theory, 4, 6, 14-19, 25, 27, 46, 122, 127

\section{H}

hypothesis, 65-67, 71, 84, 88, 106, 111, 119

\section{I}

independent and identically distributed, $8,30,35,72,107$

individual choice, 4, 6, 7, 10, 12, 24, 31, $49,58,122$

information, 2, 3, 4, 10, 11, 15-20, 22, 24-26, 28-30, 32, 34, 35, 38-47, 49, 50, 55-63, 66, 69, 70, 73-75, $77,81-84,87,88,90,92,94,102$, $113,121-126$

interaction, 3-7, 12-14, 16-18, 22-24, 30, $34,36-39,46,47,50,52,53,55$, $56,63,68-70,73,75,76,83,90$, 92, 93, 96, 107, 108, 110, 112, $121,122,124-126$

interaction effect, 68-70, 73, 75, 93, 108, 125

interactive decision, 4, 6, 13-16, 41, 50, $55,121,122,125$

interdependence, 6, 12, 13, 96, 122, 125

inter-individual, 23, 124 


\section{$\mathbf{L}$}

learning, 4, 33, 34, 38, 46, 55, 88, 89-92, $99,106,111-117,119,123,124$, 127

Log likelihood, 72, 74, 76, 78, 79, 81, 104, 109, 112

Logit, 9, 79, 81

\section{M}

management, 2, 3, 10, 11

marginal disutility, 50, 56, 93-95, 100, 101, 103-106, 108, 116-119, 123

multinomial logit model, 5, 7, 8, 31, 34, $71,72,77,78,106,110,113,115$

\section{$\mathbf{N}$}

Nash equilibrium, 4, 17, 19, 22, 25, 26, $30,32,34,35,38-42,46,54,56$, $58,59,61,62,66,67,69,84-86$, $92,95,106,108,110,111,113-$ $118,122,127$

noisy introspection, $4,18,38,47,56,57$, 60, 62, 67, 69, 84-87, 90, 91, 106, $108,111-119,122-124,127$

non-strategic, 34, 68, 69, 72-81, 84-88, 123,126

\section{$\mathbf{O}$}

one-shot, 4, 18, 35, 37, 38, 46, 56, 60, $65,67,84,88,90,91,106,108-$ $111,119,122,123,126$

optimal, 2, 11, 15, 18, 19, 24-26, 28, 33, $39,41,42,46,51,61,63,66,124$, 125,127

\section{$\mathbf{P}$}

payoff, 14, 15, 19, 20, 22, 24-32, 34, 36, $37,39,44-46,51,56,66,85,94-$ 96,125

performance, 42, 57, 58, 62, 84, 90-92, 98-101, 110, 118 prediction, $2,8,29,34,37,38,50,55,67$, $84,90,92,111$

preference, $3,6,8,9,14,15,23,24,27$, $32,37,50-53,55-57,59,66,69-$ $75,77,79,81-85,88,92-94,100-$ 106, 108-111, 116-119, 123, 126

probability, $6,8,15,18-20,25-32,35$, $36,43,44,47,50,51,54-59,61$, 69, 72, 75, 77, 80, 85, 91, 92, 95, $101,108,113,122,126$

profile, 19-22, 25-27, 29, 70, 73, 100

projection, $3,84,90,91,108,110,111$, $113-117,119,123,124$

\section{Q}

quantal response equilibrium, 4, 18, 27, $29,34,46,55,56,59,61,62,66$, $67,69,85,87,106,108,110-116$, 118,122

\section{$\mathbf{R}$}

random coefficients model, 71-74, 77, 79,81

rational, $14,16,18,24,28,29,32,35-$ $38,47,66,92,124,126$

reaction, $4,11,26,39,41,42,50,63,82$, $107,112,113,116,124$

recommendation, $11,39,41-45,56-60$, 62, 63, 88, 90-103, 106-116, 118, $119,122-126$

regression model, 90, 98, 103, 104, 111, 118

repeated choice, 4, 18, 33-36, 38, 47, 56, 71, 88, 89-93, 96, 98, 103, 106, $107,111,113-119,122,123,126$

response, $1,4,10,11,12,15,18,22,25$ $30,34,36,38,39,41-44,46,47$, $55,56,59-62,66,67,69,84,85$, 87, 91, 106, 108, 110-114, 116, $118,122,124-126$

Rho-squared, 75, 76, 80, 82, 84, 109, 111 
$\mathbf{S}$

scale parameter, $31,35,87,126$

scenario, 51, 53, 57, 58, 68, 76-78, 83, 91

simulation, 9, 47, 49-51, 54, 55, 57, 62, $63,85,86,92,94,108,122$

spatial choice, 5, 6, 9, 14, 16, 17, 37, 38

Stackelberg equilibrium, 39, 41, 42, 47, 49, 122

strategic behaviour, 3, 16, 17, 79, 81, 89, $90,92,93,121,122,126$

strategic choice, 2-4, 14, 16-18, 22, 27, 29, 31, 33, 34, 38, 39, 46, 47, 49, $50,55,63,65,67,69,70,72,73$, $76,84,89,90,92-95,98,101,107-$ $109,111,112,114,118,119,122-$ 124

strategic equilibrium, 19, 24, 27, 87

strategic situation, 15, 24, 33-35, 39, 46, 47, 67, 77, 79-81, 84, 88, 90, 91, $122-125,127$

strategy, 14, 18, 21, 22, 24-29, 32, 35, 39-44, 54, 58, 61, 62, 66, 85, 91, 92,108

$\mathbf{T}$

timing, 2-4, 9, 13, 18, 19, 34, 37-39, 51, $58,65-84,86-89,93,123$

$\mathbf{U}$

uncertainty, $3,10,11,14,16,18-20,25$, 28-33, 41, 51, 54, 62, 65, 69, 72, 79, 80, 90-94, 99, 100, 103, 117$119,123,124,126$

utility, 2, 5-9, 11, 12, 15, 17-26, 28, 29, $31-35,37,40,43,45,46,50,53$, $56,66,69,71-73,75,77,80,85$, 87, 94, 103, 107-111, 114, 117, $122,124,126$

utility function, 12, 18-20, 22, 23, 40, 45, 136,140
V

validity, 4, 6, 8, 47, 49, 50, 63, 65, 123

\section{W}

welfare maximum, 41, 57, 59, 61, 62, 94 


\section{Samenvatting (Dutch summary)}

Temporele variatie in de vraag naar faciliteiten met capaciteitsbeperkingen kan leiden tot congestie. In een dergelijke situatie kunnen de aanbieders van voorzieningen en individuele gebruikers strategisch gedrag vertonen. Individuele gebruikers passen wellicht hun gedrag aan om congestie te vermijden. Stedelijke autoriteiten en aanbieders van voorzieningen moeten de reacties van gebruikers voorspellen om bepaalde doelstellingen, zoals een optimaal gebruik van de beschikbare beperkte faciliteiten of het vermijden van congestie, te realiseren. Bestaande ruimtelijke keuzemodellen houden geen rekening met dergelijk strategisch gedrag.

In dit proefschrift wordt daarom een modelbenadering ontwikkeld, die stedelijke autoriteiten en aanbieders van diensten kan ondersteunen in het voorspellen van dergelijk strategisch gedrag van individuen en in het evalueren van de effecten van informatievoorziening op de verdeling van de vraag. Op grond van deze voorspellingen zouden de betreffende actoren de bestaande voorzieningen (infrastructuur, openbare ruimte, culturele voorzieningen, nieuwe technologie, etc.) beter moeten kunnen beheren. In methodisch opzicht kan de ontwikkelde benaderingswijze worden beschouwd als een combinatie van discrete keuze modellen en speltheorie, zodanig dat de interacties en wederzijdse afhankelijkheden tussen gebruikers onderling en tussen gebruikers en informatieverschaffers worden meegenomen.

De theoretische basis is die van een $N$-speler $J$-opties spel, waarbij een discreet keuze model met een endogene interactie-term wordt gebruikt om strategisch keuzegedrag van een gebruiker te beschrijven, waarbij de keuzes van alle andere gebruikers verklarende variabelen zijn in de nutsfunctie. Drie situaties worden onderscheiden: (1) verwachting met volledige informatie in ideale situaties - Nash 
evenwicht; (2) verwachting met onvolledige informatie in herhaalde situaties - quantal response evenwicht, en (3) verwachting met onvolledige informatie in enkelvoudige situaties - noisy introspectie. Een logit response functie wordt gebruikt om de verwachtingen over strategieën te transformeren in de kans op bepaald keuzegedrag.

Dit basis model is vervolgens uitgebreid om het probleem van de interactie tussen de informatieverschaffer en het collectief van gebruikers op te lossen. Hiertoe is een spel geformuleerd met 2-spelers en 2-fases met een Stackelberg evenwicht. Drie typische doelstellingen/rollen voor de informatieverschaffer zijn hierbij onderscheiden: (1) facility manager met als doel een gelijkmatig, optimaal gebruik over de reeks van tijdsperioden, (2) overheidsinstantie met als doel maximalisatie van welvaart voor het totaal van gebruikers, en (3) een representant van het collectief van gebruikers met als doel een gelijke verdeling van nut over individuen.

De eigenschappen van de ontwikkelde modellen zijn eerst systematisch verkend door een serie numerieke simulaties uit te voeren, waarbij parameters zijn gevarieerd. De resultaten van deze Monte Carlo simulaties leverden bewijs voor de validiteit van de modellen. Vervolgens zijn de modellen empirisch geschat op grond van een tweetal interactieve computer experimenten over temporele voorkeuren in verschillende toepassingsgebieden. De resultaten van deze experimenten geven aan dat gebruikers inderdaad strategisch gedrag vertonen en hun voorkeuren aanpassen aan de hand van hun verwachtingen over het strategisch gedrag van andere gebruikers om congestie te vermijden.

Een tweede studie ging met name in op de mogelijkheid van leergedrag van gebruikers. De resultaten laten opnieuw zien dat gebruikers strategisch gedrag vertonen, en ook dat men leert van de gevolgen van hun beslissingen en hun gedrag successievelijk aanpast om congestie te vermijden. Bovendien laten de resultaten van de experimenten zien dat strategisch gedrag inderdaad verschilt tussen de situatie waarin informatie wordt gegeven en de situatie waarin dat niet gebeurd. De effecten van marginaal negatief nut en het volgen van de informatie/aanbeveling neemt toe naarmate gebruikers leren, terwijl het effect van onzekerheid over de voorkeuren van anderen niet toeneemt.

Het noisy introspectie model, gebaseerd op de veronderstelling van iteratieve redenatie met een toenemende fout, geeft de beste beschrijving van de experimentele data, zowel met als zonder aanbevelingen.

Het belangrijkste resultaat en de bijdrage van het project is de ontwikkeling van modellen van strategisch keuzegedrag. Geen van deze modellen is direct toepasbaar op een concreet probleem. Ontwikkeld zijn model-alternatieven voor specifieke klassen van strategisch gedrag. Door middel van nadere operationalisatie en specifieke dataverzameling zijn deze modellen echter wel bruikbaar te maken voor verschillende problemen waarin strategisch gedrag en onzekerheid een cruciale rol spelen. Voorbeelden zijn persoonlijke informatie-systemen, reisgedrag en het optimaal gedrag van infrastructuur met maximaal comfort, zogenaamde location-based services, en optimaal gebruik van parkeerlocaties; vele andere voorbeelden op verschillende vakgebieden zijn te noemen. 


\section{Curriculum vitae}

Qi Han was born in 1971 in Nanjing, China. She studied Architecture at Tongji University, China and obtained her Bachelor's Degree in Architecture in 1995. After graduation, she worked as an engineer in the East China Electric Power Design Institute. From 1998 to 2000, she completed her Master degree study in Architecture and Building Science of Tohoku University, Japan.

Currently, she is a post doctorate research assistant of the Urban Planning Group at the Eindhoven University of Technology, The Netherlands. She was a Ph.D. candidate at the same university from 2001 to 2005.

Her current research interests are in developing models and experiments for dynamic consumer behaviour and choice processes in marketing, retailing, tourism, transportation and urban planning. 